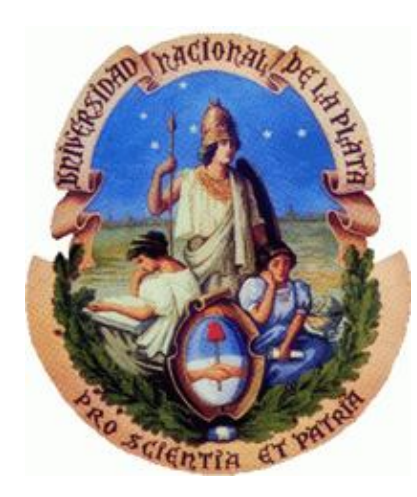

UNIVERSIDAD NACIONAL DE LA PLATA FACULTAD DE CIENCIAS EXACTAS DEPARTAMENTO DE QUIIMICA

\author{
Tesis Doctoral
}

\title{
Propiedades fotoquímicas de biopterina, neopterina y sus correspondientes dihidroderivados en solución acuosa bajo irradiación UV-A
}

Licenciada en Bioquímica Mariana Vignoni Director: Dr. Andrés H. Thomas Co-director: Dr. Franco M. Cabrerizo

2011 

El presente trabajo de Tesis se realizó en el Instituto de Investigaciones Fisicoquímicas Teóricas y Aplicadas bajo la dirección del Dr. Andrés $\mathrm{H}$. Thomas y la codirección del Dr. Franco M. Cabrerizo. Se presenta a consideración de las autoridades de la Facultad de Ciencias Exactas, de la Universidad Nacional de La Plata a fin de acceder al Grado Académico de Doctor de la Facultad de Ciencias Exactas. 

Quiero agradecer a todas las personas que hicieron posible este Trabajo de Tesis, especialmente:

Al Dr. Andrés Thomas, director de este Trabajo de Tesis, por toda su ayuda a lo largo de estos años, a cualquier hora y día de la semana, por la paciencia (casi) infinita, por enseñarme que las cosas no son fáciles, pero se puede.

Al Dr. Franco Cabrerizo, co-director de este Trabajo de Tesis, por haberme traído al grupo hace ya más de 8 años. Por enseñarme a trabajar en el laboratorio y por su gran ayuda durante todo este tiempo.

A "las chicas", Laura, Paula, Caro, Gaby y Mariana P., porque siempre me ayudaron en todo lo que necesité, por todos los buenos momentos, congresos, mates, facturas y chocolates compartidos (incluyo a Andrés en esta última parte). No podría haberlo hecho sin Uds.

A la Dra. Rosa Erra-Balsells por haberme permitido utilizar el equipo de RMN y por toda su ayuda con la interpretación de los resultados.

A la Dra. María Laura Salum, por su ayuda con los experimentos de RMN.

A la Dra. Esther Oliveros, directora del Laboratoire des IMRCP de la Université Paul Sabatier, por permitirme utilizar su equipamiento, por ayudarme con la interpretación de los resultados y por toda su ayuda durante mi estadía en Toulouse.

A la Dra. Patricia Vicendo por su ayuda con los experimentos de EPR.

A toda la gente del INIFTA, especialmente a los del primer piso, por los gratos momentos compartidos.

A Esther, André, Maite, Patricia y toda la gente del IMRCP por hacer que de mi estadía en Toulouse sea inolvidable.

A todos mis amigos internacionales que conocí en Argentina, Francia y Alemania.

A mis amigos de siempre, por aguantarme desde hace años (no se que hubiese hecho sin Uds.).

A mi familia por ayudarme, soportarme y apoyarme en todas mis decisiones, y por todo el amor que siempre me dan.

Al Consejo Nacional de Investigaciones Científicas y Técnicas (CONICET), por las becas de Postgrado Tipo I (2007-2009) y Tipo II (2010-2011) ambas para realizar el Doctorado. 
A las autoridades de la Facultad de Ciencias Exactas por haber accedido a que realizara mis actividades en esta institución, en particular, a las autoridades del Instituto de Investigaciones Fisicoquímicas Teóricas y Aplicadas (INIFTA).

A la Agencia Nacional de Promoción de la Ciencia y Tecnología (ANPCyT), al Consejo Nacional de Investigaciones Científicas y Técnicas (CONICET) y a la Universidad Nacional de La Plata (UNLP) por financiar los proyectos de investigación que dieron marco al presente Trabajo de Tesis.

Al Ministerio de Ciencia, Tecnología e Innovación Productiva (MINCyT, Argentina) and ECOS-Sud (Francia), en el marco del proyecto A07E07, por financiar mi estadía en la Universidad Paul Sabatier de Toulouse, Francia. 


\section{Índice}

\section{Prólogo}

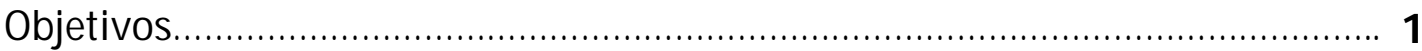

Estructura y diseño del Trabajo de Tesis................................................... 1

\section{PARTE I: INTRODUCCIÓN}

1 Introducción a la fotofísica y fotoquímica........................................................ 3

1.1 Procesos fotofísicos........................................................................... 3

1.2 Procesos fotoquímicos....................................................................... 6

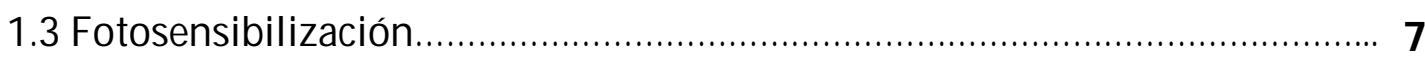

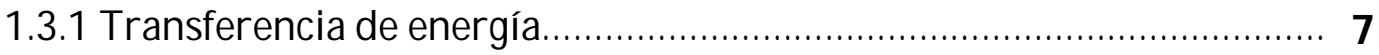

1.3.2 Transferencia de electrones........................................................ 9

1.4 Oxidaciones fotosensibilizadas........................................................... 9

1.4.1 Mecanismos Tipo I.................................................................................... 10

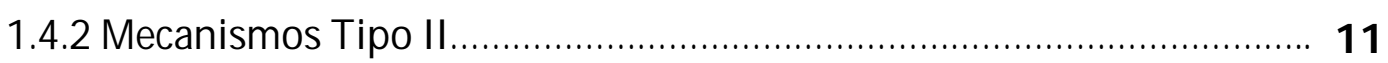

1.5 Referencias.............................................................................................11

2 Propiedades químicas y bioquímicas de pteridinas.......................................13

2.1 Estructura química...........................................................................13

2.2 Propiedades ácido-base y espectros de absorción......................................15

2.3 Reactividad química de pterinas...........................................................19

2.4 Bioquímica de biopterina y sus derivados reducidos..........................................21

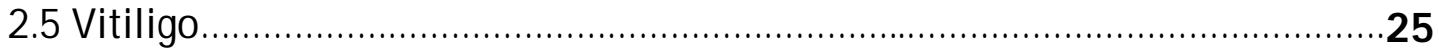

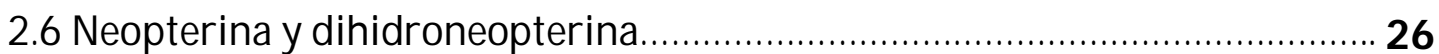

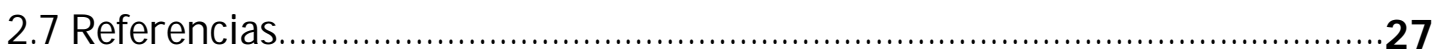

3 Fotoquímica de pterinas........................................................................................ 31

3.1 Estados excitados singletes y tripletes de pterinas..................................... 31

3.2 Reactividad fotoquímica de las pterinas aromáticas.....................................34

3.2.1 Pterinas sin sustituyente oxidable.................................................... 34

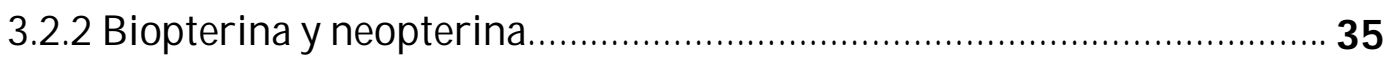

3.2.3 Formilpterina e hidroximetilpterina...........................................35

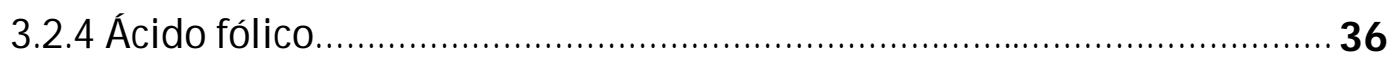

3.3 Reactividad fotoquímica de los derivados reducidos.................................. 37 


\section{PARTE II: MATERIALES Y MÉTODOS EXPERIMENTALES}

4 Preparación de soluciones y metodología de trabajo................................41

4.1 Diseño general de los experimentos..............................................41

4.2 Reactivos y preparación de soluciones de pterinas................................ 42

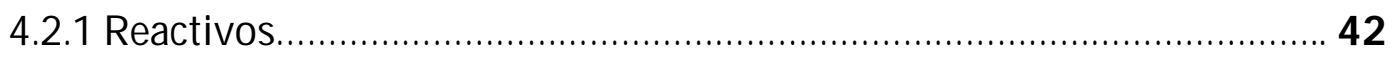

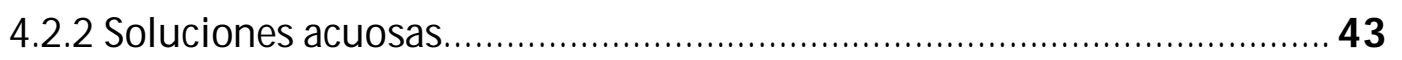

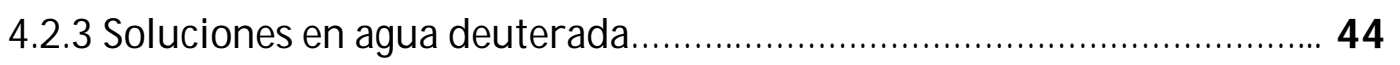

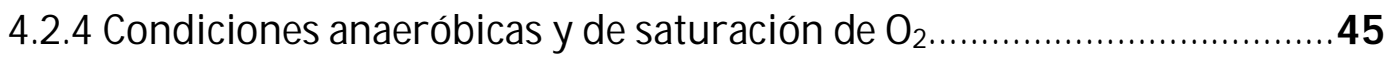

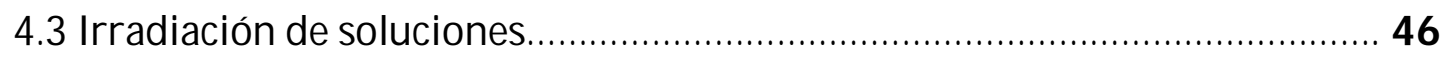

4.4 Determinación de rendimientos cuánticos...........................................48

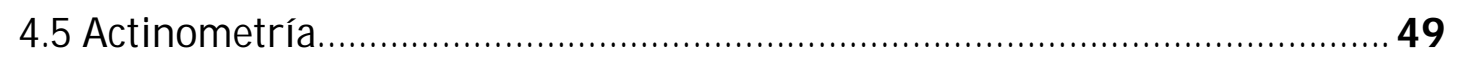

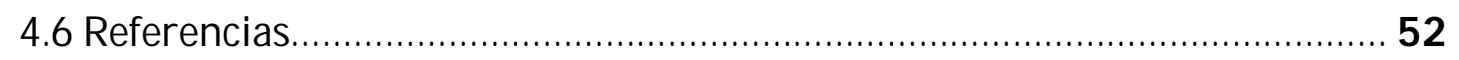

5 Técnicas analíticas................................................................................. 53

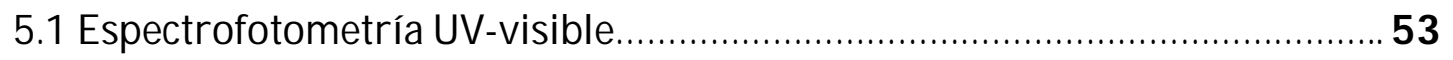

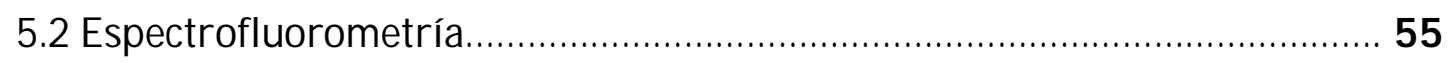

5.3 Cromatografía Líquida de Alto Rendimiento.......................................55

5.4 Espectrometría de masa...........................................................59

5.5 Resonancia Magnética Nuclear.....................................................61

5.6 Resonancia Paramagnética Electrónica...............................................63 63

5.7 Detección de peróxido de hidrógeno...............................................64

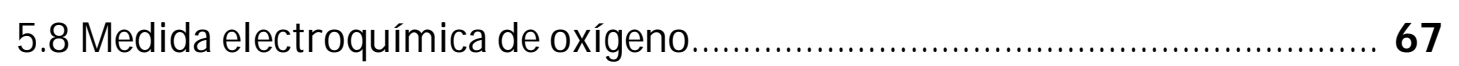

5.9 Detección de anión superóxido por Citocromo c...................................68 68

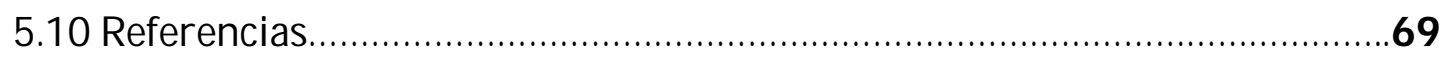

\section{PARTE III: RESULTADOS}

6 Fotoquímica de biopterina y neopterina......................................................... 71

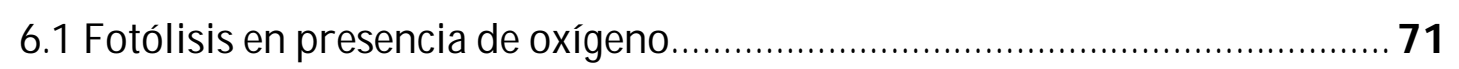

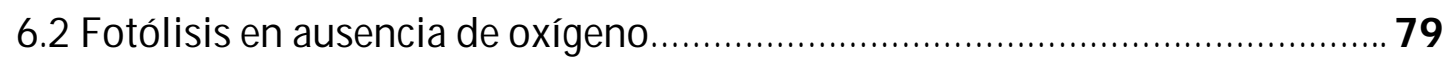

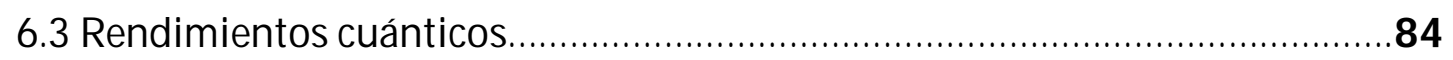

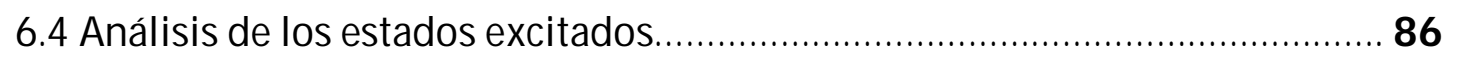

6.5 Investigación de anión superóxido.................................................88

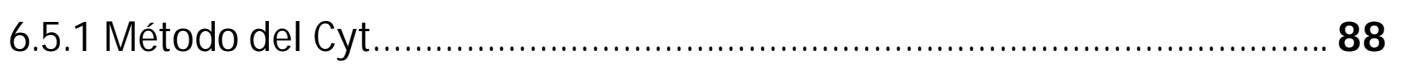


6.5.2 Resonancia Paramagnética Electrónica............................................ 91

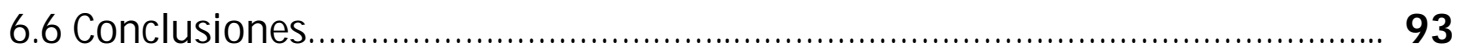

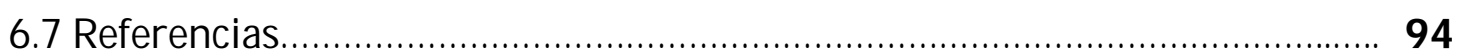

7 Caracterización del Intermediario Rojo......................................................... 97

7.1 Análisis de la estructura del intermediario por RMN............................... 97

7.2 Análisis de los fotoproductos de 6-hidroximetilpterina por RMN................ 103

7.3 Estudio de la estabilidad del intermediario............................................ 105

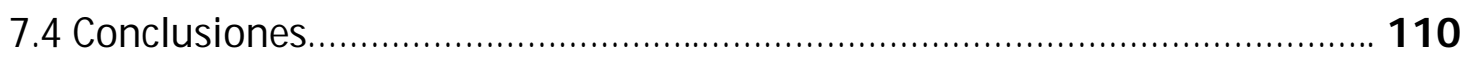

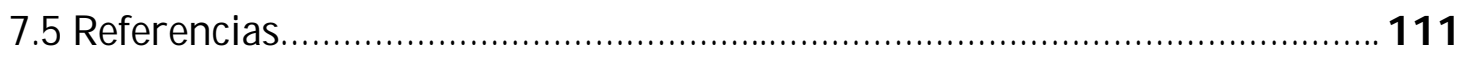

8 Fotoquímica de 7,8-dihidroneopterina..........................................................113

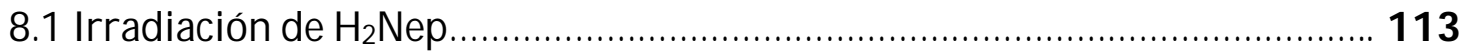

8.2 Análisis de los productos por espectrometría de masa................................ 118

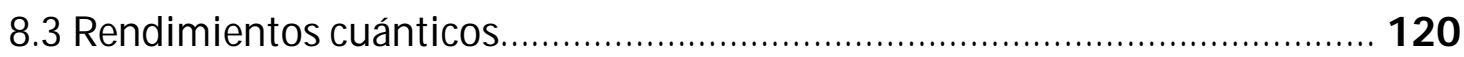

8.4 Análisis del mecanismo de reacción..................................................121

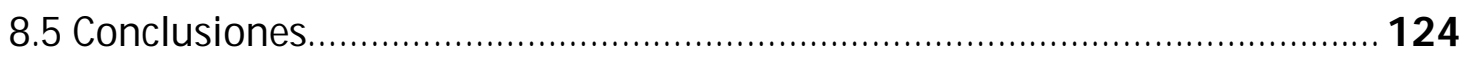

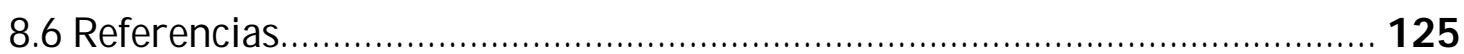

9 Fotoquímica de 7,8-dihidrobiopterina....................................................... 127

9.1 Irradiación en ausencia de oxígeno...................................................127

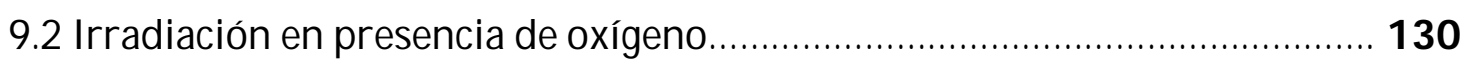

9.3 Análisis de los productos por espectrometría de masa..............................140

9.4 Implicancias biológicas de las propiedades fotoquímicas de $\mathrm{H}_{2}$ Bip...............144

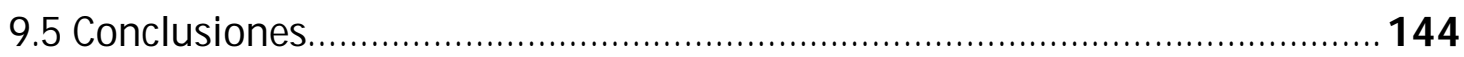

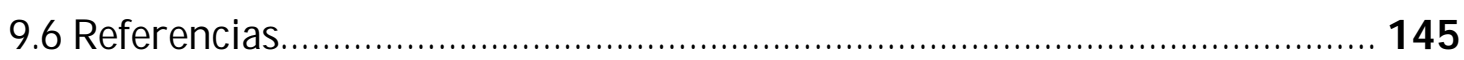

10 Oxidación fotosensibilizada de dihidropterinas.......................................147

10.1 Análisis de la participación de los estados excitados............................... 147

10.2 Irradiación de mezclas $\mathrm{H}_{2}$ Bip y Bip......................................................151

10.3 Irradiación de mezclas $\mathrm{H}_{2}$ Bip con otras pterinas oxidadas........................ 154

10.4 Análisis del mecanismo de reacción....................................................157

10.5 Investigación de la oxidación fotosensibilizada de $\mathrm{H}_{2} \mathrm{Nep}$.........................160

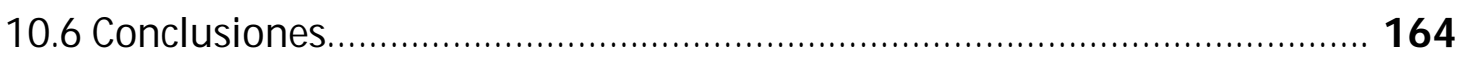

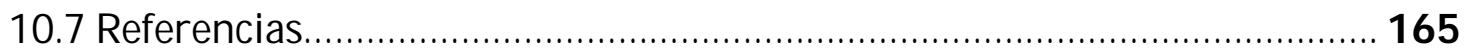

11 Caracterización y estabilidad térmica de los fotodímeros.........................167

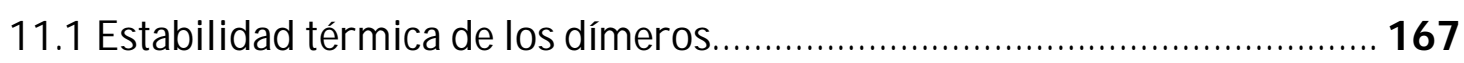

11.2 Caracterización de P1 por espectrometría de masa.................................171 
11.3 Análisis de la estructura de los dímeros. 173

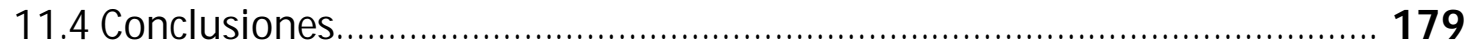

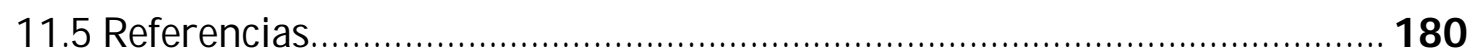

Conclusiones Generales...................................................................................... 181 


\section{Prólogo}

\section{Objetivos}

El presente trabajo de Tesis Doctoral consiste en un estudio de la fotoquímica de biopterina (Bip), neopterina (Nep), 7,8-dihidrobiopterina $\quad\left(\mathrm{H}_{2} \mathrm{Bip}\right)$ y $\quad$ 7,8dihidroneopterina ( $\mathrm{H}_{2} \mathrm{Nep}$ ), en solución acuosa bajo irradiación UV-A (320 - $\left.400 \mathrm{~nm}\right)$. A continuación se mencionan los objetivos específicos que han dirigido el desarrollo de este trabajo de tesis:

- Establecer las reacciones fotoquímicas que sufren las pterinas escogidas, midiendo las eficiencias cuánticas e investigando los fotoproductos generados.

- Dilucidar los mecanismos de reacción bajo distintas condiciones experimentales, ya sea a diferentes $\mathrm{pH}$ o distintas concentraciones de $\mathrm{O}_{2}$.

- Investigar la formación de especies reactivas de oxígeno (EROs), particularmente anión superóxido y peróxido de hidrógeno.

- Investigar la estabilidad de los fotoproductos formados y las reacciones térmicas que los mismos sufren bajo distintas condiciones experimentales.

\section{Estructura y diseño del Trabajo de Tesis}

El presente trabajo de Tesis doctoral se divide en tres partes, cada una de las cuales está subdividida, a su vez, en varios capítulos. A continuación se presentará un breve resumen sobre el contenido de cada una de las partes.

Parte I: Introducción. Posee tres capítulos en los cuales se encuentran los antecedentes relevantes publicados sobre las propiedades generales y fotoquímicas de las pterinas. El Capítulo 1 consiste en un breve desarrollo acerca de los conceptos elementales de fotofísica y fotoquímica, necesarios para una fácil lectura y correcta interpretación de este trabajo. El Capítulo 2 incluye la nomenclatura básica y propiedades fisicoquímicas de los compuestos estudiados, además de una síntesis de la participación de las pterinas en los sistemas biológicos en condiciones fisiológicas y patológicas. El Capítulo 3 resume la información accesible en literatura acerca del comportamiento fotoquímico y fotofísico de las pterinas en general y de los derivados estudiados en este trabajo, en particular. 
Parte II: Materiales y métodos experimentales. En esta parte, se detalla la metodología de trabajo y las técnicas experimentales utilizadas.

Parte III: Resultados. Esta parte corresponde a la presentación, análisis y discusión de los resultados experimentales obtenidos. En el Capítulo 6 se exponen los resultados generales de las reacciones de fotólisis que sufren las pterinas oxidadas, Bip y Nep. En estas reacciones se detectó un intermediario, cuya estructura y reactividad se discute en el Capítulo 7. Los Capítulos 8 y 9 corresponden a los resultados del estudio de la fotoquímica de $\mathrm{H}_{2} \mathrm{Nep}$ y $\mathrm{H}_{2} \mathrm{Bip}$, respectivamente. Estos dos compuestos, a diferencia de las pterinas oxidadas, se presentan en capítulos separados porque su comportamiento fotoquímico es muy diferente. En el Capítulo 10 se presentan resultados experimentales tendientes a dilucidar mecanismos que explican las reacciones presentados en los Capítulos 8 y 9 y que involucran procesos fotosensibilizados. Finalmente, el Capítulo 11 analiza la estructura química y estabilidad de los fotoproductos formados por las dihidropterinas. 


\section{PARTE I}

\section{INTRODUCCIÓN}





\section{Capítulo 1}

\section{Introducción a la fotofísica y fotoquímica}

Cuando una molécula absorbe radiación electromagnética se produce la excitación desde un estado cuántico de menor energía a otro de mayor energía. En este estado electrónico excitado, la molécula se encuentra en una situación muy inestable respecto de su estado basal, motivo por el cual puede perder el exceso de energía de diferentes modos. Si la molécula sufre una transformación química, entonces se denomina proceso fotoquímico. En cambio, si permanece químicamente inalterada y se desactiva mediante alguna forma física, el proceso es fotofísico.

\subsection{Procesos fotofísicos}

Existen muchos caminos de relajación física posibles y el más favorable dependerá del tipo de molécula, de la naturaleza de los estados electrónicos involucrados y de las propiedades del medio. Estas vías de desactivación suelen ser muy rápidas y pueden clasificarse de la siguiente manera:

$\checkmark \quad$ Transiciones radiativas, en las cuales la molécula excitada emite radiación electromagnética para regresar al estado de menor energía. Esta energía emitida es menor que la utilizada para generar el estado excitado.

$\checkmark \quad$ Transiciones no radiativas, en las cuales un estado previamente excitado se convierte en otro de menor energía, sin emisión de radiación, por transferencia intramolecular de energía.

$\checkmark \quad$ Procesos de desactivación física que involucran la transferencia de energía de la molécula inicialmente excitada a otras moléculas que se encuentran presentes en el medio.

Cada una de estas vías de desactivación física puede involucrar, a su vez, distintos procesos. En el Esquema 1.1 se muestra un diagrama de Jablonski modificado donde se representan las transiciones radiativas y no radiativas que puede sufrir una molécula. En este esquema simplificado de niveles de energía, las transiciones radiativas se representan con flechas de línea recta y las transiciones no radiativas con flechas de 
líneas onduladas. Los niveles vibro-rotacionales asociados a cada estado electrónico se representan con líneas horizontales.

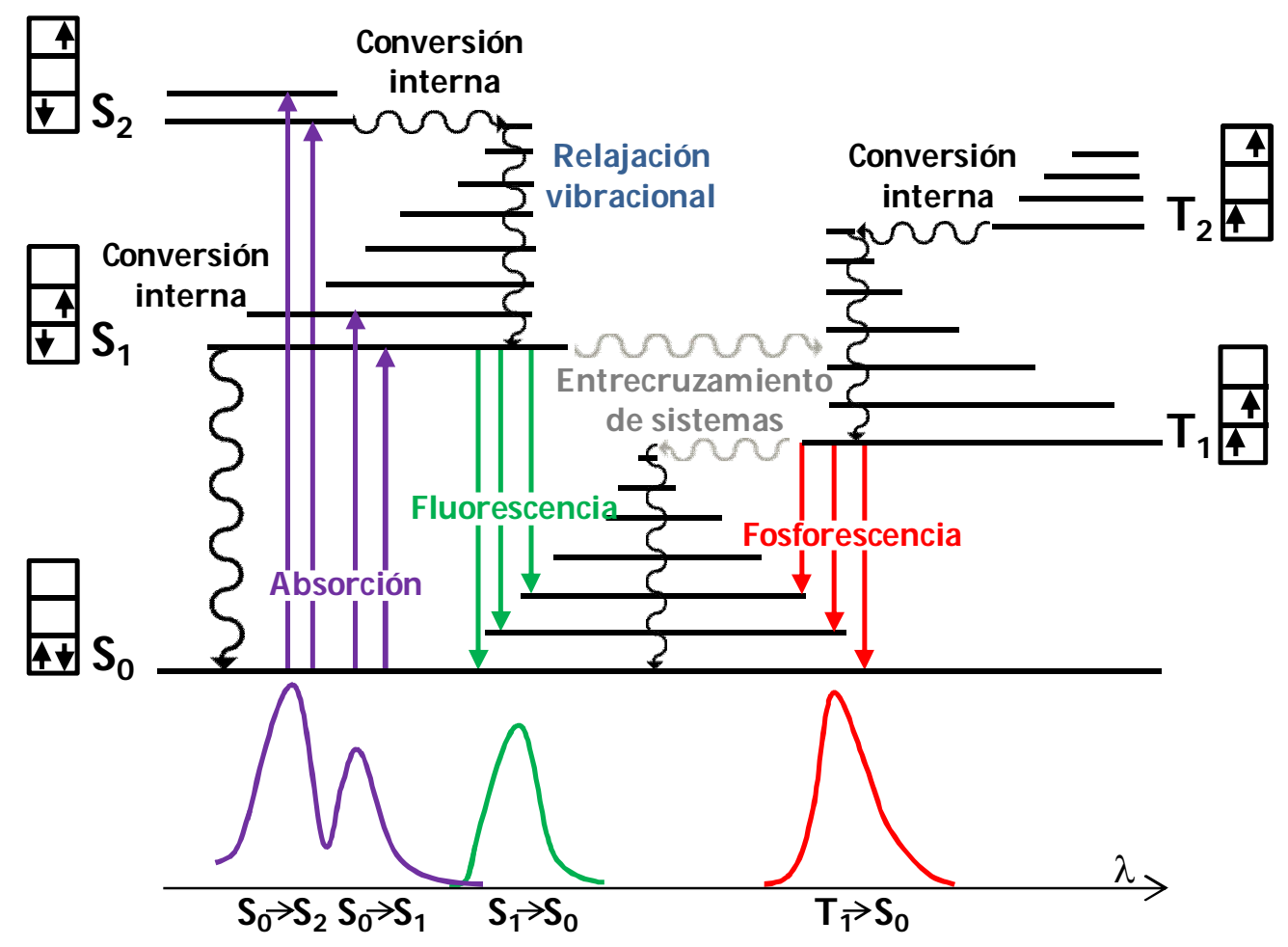

\section{Esquema 1.1 Diagrama de Jablonski modificado.}

Típicamente, las moléculas orgánicas se encuentran en un estado electrónico basal de tipo singlete $\left(\mathrm{S}_{0}\right)$. Cuando se produce la absorción de radiación electromagnética se promueve un electrón a un orbital de mayor energía. Si no hay cambio en la multiplicidad de spin, el estado electrónico excitado alcanzado continúa siendo singlete $\left(\mathrm{S}_{1}\right)$. En cambio, si ocurre un cambio en la multiplicidad de spin el estado electrónico alcanzado será de tipo triplete $\left(\mathrm{T}_{1}\right)$. Este último estado posee menor energía que el estado excitado $\mathrm{S}_{1}$.

Cuando se produce un proceso de excitación electrónica siempre existe una preferencia sobre la conservación del spin, motivo por el cual las bandas de absorción más intensas en un espectro corresponden a transiciones del tipo $\mathrm{S}_{0} \rightarrow \mathrm{S}_{1}$. En cambio, las transiciones $\mathrm{S}_{0} \rightarrow \mathrm{T}_{1}$ están "prohibidas por spin", es decir, que tienen una probabilidad de ocurrencia extremadamente baja, lo que deriva en transiciones muy débiles. Usualmente, la excitación al primer estado excitado $\left(\mathrm{S}_{1}\right)$ es el proceso más favorable. Sin embargo, muchas moléculas pueden absorber radiación a un segundo estado excitado singlete de mayor energía presentando transiciones $\mathrm{S}_{0} \rightarrow \mathrm{S}_{2}$. 
Como se observa en el diagrama de Jablonski, las transiciones no radiativas pueden ocurrir entre estados de igual multiplicidad o entre estados de distinta multiplicidad. A los primeros se los denomina procesos de conversión interna (IC, internal convertion) y a los últimos, entrecruzamiento de sistemas (ISC, intersystem crossing). Las transiciones horizontales entre estados, por IC o ISC, dejan a la molécula con un exceso de energía vibro-rotacional. En solución esta energía es rápidamente removida por colisiones con moléculas del solvente, en un proceso denominado relajación vibracional (VR).

Los procesos radiativos que pueden ocurrir se denominan fluorescencia y fosforescencia. En ambos casos, la emisión de la radiación sucede a longitudes de onda mayores que la radiación absorbida inicialmente. Esto se debe a que el estado excitado inicialmente formado se equilibra térmicamente para alcanzar el menor estado electrónico vibro-rotacional dentro de dicho estado electrónico excitado. ${ }^{1} \mathrm{Si}$ la transición ocurre desde un estado electrónico de igual multiplicidad de spin que el estado final, la emisión se denomina fluorescencia. La misma es una transición fuertemente permitida y muy rápida. La fosforescencia, en cambio, es una transición que involucra estados de diferente multiplicidad de spin, es decir, una transición prohibida en términos de la teoría mecano-cuántica. No obstante, esta puede ocurrir y, generalmente, es de menor intensidad y más lenta que la fluorescencia. Pueden evaluarse experimentalmente las propiedades de la radiación emitida para conocer detalles de la naturaleza y el comportamiento de los estados excitados.

Otra vía de relajación del estado excitado consiste en el quenching o desactivación física por otra molécula. Esta vía está presente cuando se encuentra, en el sistema o medio de reacción, otra especie química capaz de interactuar con el estado excitado de la molécula que absorbió, en primera instancia, la radiación electromagnética. Esta segunda molécula se conoce, usualmente, como desactivador o quencher. De esta manera, una molécula excitada puede interaccionar con el desactivador dando lugar a la relajación sin emisión de radiación. En este proceso generalmente está involucrada la transferencia de energía de la molécula excitada a la otra molécula en una colisión. De esta manera, la intensidad de la radiación se reduce en una cantidad dependiente de la concentración del desactivador y de la eficiencia del proceso de desactivación. Además, la presencia del desactivador aumenta la velocidad del decaimiento de la emisión. Por lo tanto, medidas de la intensidad de la emisión y su dependencia en el tiempo proveen 
información acerca de las velocidades de reacción entre la molécula excitada y el desactivador. ${ }^{2}$

\subsection{Procesos fotoquímicos}

Cuando una molécula absorbe radiación electromagnética, la misma queda en un estado de mayor energía que aumenta las posibilidades de reacción respecto de la misma molécula en estado basal. ${ }^{3}$ Este exceso de energía puede emplearse para promover una reacción química generando entonces una reacción fotoquímica. Esto ocurre por distintos mecanismos: la energía puede ser utilizada para romper una unión química, para superar una energía de activación, etc. Queda claro que la reactividad química de una molécula en un estado excitado puede ser muy diferente a la reactividad química de esa misma molécula en su estado electrónico fundamental.

Según el tipo de transformación química las reacciones fotoquímicas pueden clasificarse en (Esquema 1.2):

$\checkmark \quad$ Reacciones de disociación: la energía de la radiación debe ser suficiente para producir la ruptura de un enlace, dando lugar a la fragmentación de la molécula excitada.

$\checkmark \quad$ Reacciones o procesos intermoleculares: la excitación de una molécula favorece la reacción con una segunda molécula, reacción que no tiene lugar desde el estado electrónico fundamental. Pueden ser reacciones directas entre la molécula excitada y una segunda molécula, o procesos de transferencia de electrones entre ambas moléculas generando especies radicales.

$\checkmark \quad$ Procesos intramoleculares: en un estado excitado una parte de una molécula interacciona con otra parte de la misma molécula. Estos procesos conducen a cambios estructurales de la molécula y/ o procesos de isomerización.

$\checkmark \quad$ Procesos de ionización: la energía de la radiación produce la separación de un electrón $\mathrm{y}$, por lo tanto, la ionización de la molécula excitada. La molécula ionizada posteriormente sufre una transformación química, por ejemplo, por reacción con otra molécula. 


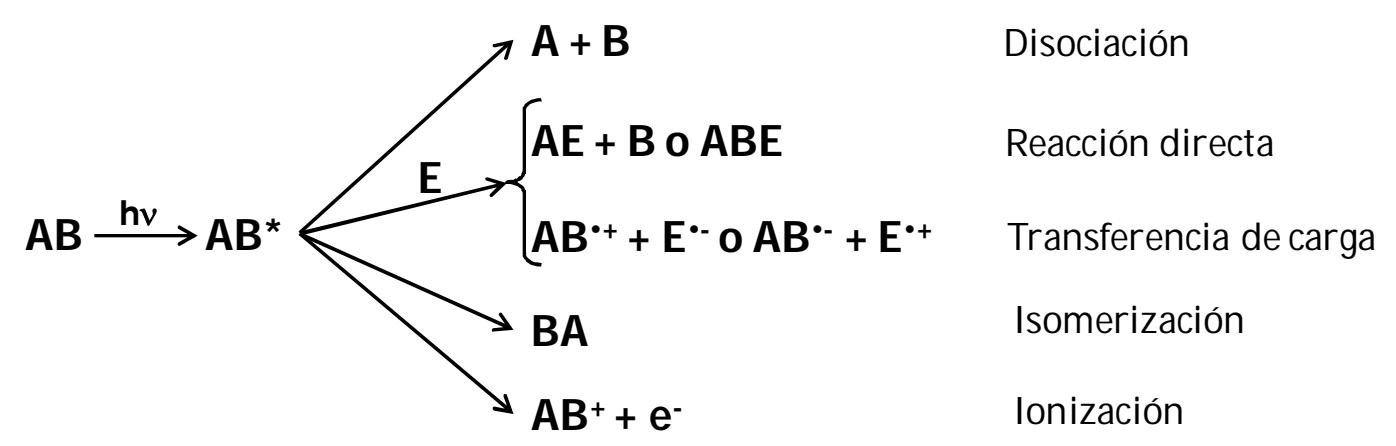

Esquema 1.2 Esquema de los distintos procesos fotoquímicos descriptos previamente.

\subsection{Fotosensibilización}

La fotosensibilización abarca un conjunto especial de procesos fotoquímicos en los cuales, una especie química sufre una alteración fotoquímica o fotofísica como resultado de la absorción inicial de radiación electromagnética por otra especie química que se denomina fotosensibilizador 0 , simplemente, sensibilizador (Sens) ${ }^{4,5} \mathrm{Si}$ bien ésta es una definición amplia, y existen otras más acotadas, es también la más aceptada en el campo de la fotoquímica aplicada a reacciones de compuestos orgánicos y procesos bioquímicos.

Según el mecanismo, los procesos fotosensibilizados pueden ocurrir a través de transferencia de energía o transferencia de carga. Ambos mecanismos se detallan a continuación.

\subsubsection{Transferencia de energía}

La fotosensibilización por transferencia de energía es un proceso en el cual una especie química electrónicamente excitada ("dador") se desactiva transfiriendo energía a una segunda especie química ("aceptor"). Como consecuencia, esa energía produce en el aceptor una transición electrónica poblando estados excitados (generalmente los de más baja energía, $\mathrm{S}_{1}$ o $\mathrm{T}_{1}$ ) que luego se transforma en algún fenómeno químico o físico.

En el Esquema 1.3 se presenta la secuencia de reacciones involucradas en este mecanismo. Primero, el sensibilizador (Sens) obtiene la energía de excitación a partir de la absorción de un fotón generando un estado excitado (Sens*). Luego, la energía se transfiere al aceptor (A) y las moléculas excitadas del sensibilizador regresan al estado basal. Esta energía transferida es menor a la absorbida inicialmente debido a la relajación o pérdida de energía vibro-rotacional. Posteriormente, $\mathrm{A}^{*}$ puede volver a su 
estado fundamental desactivándose a través de cualquiera de las vías fotofísicas descriptas en la Sección 1.1 o sufrir alguno de los cambios químicos presentados en la Sección 1.2 .

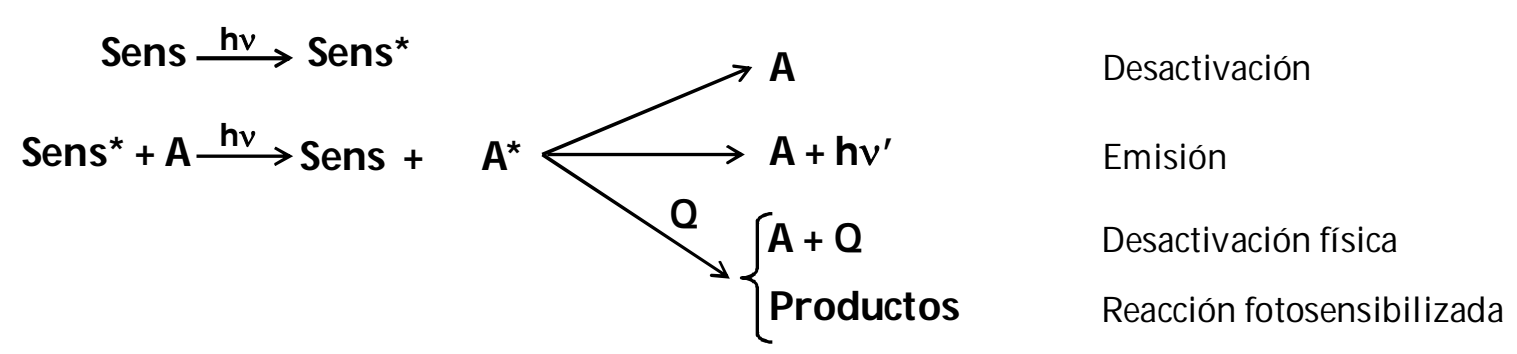

\section{Esquema 1.3 Proceso fotosensibilizado que ocurre a través de mecanismos de transferencia de energía.}

La transferencia de energía genera la excitación de la especie que no absorbe la radiación electromagnética, lo cual puede traducirse en cambios químicos sufridos por el aceptor que no ocurrirían en ausencia de radiación y del sensibilizador. Por otro lado, esta vía alternativa de excitación puede poblar estados excitados distintos a los obtenidos por absorción directa y, por lo tanto, puede dar lugar a distintas reacciones químicas. ${ }^{6}$

La transferencia de energía puede ocurrir, a su vez, a través de dos mecanismo distintos: ${ }^{7}$

$\checkmark \quad$ Transferencia de energía radiativa: la energía se transfiere por desactivación radiativa de la molécula dadora (Sens) y absorción de la radiación emitida por una molécula aceptora (A). ${ }^{4}$

$\checkmark \quad$ Transferencia de energía no radiativa: requiere una interacción directa entre Sens* y A.

La transferencia de energía triplete-triplete es el tipo más frecuente y más importante en fotoquímica orgánica. La misma es utilizada comúnmente para generar estados excitados tripletes de moléculas con bajos rendimientos cuánticos de producción de este tipo de estados excitados. Es decir, existen compuestos que no pueden generar estados tripletes por absorción directa de radiación pero que, sin embargo, pueden generarlos aceptando energía de un fotosensibilizador excitado. La eficiencia del proceso de transferencia de energía depende de las configuraciones de spin y de la energía relativa de los estados excitados del dador y del aceptor. Otro caso importante de transferencia de energía es la excitación fotosensibilizada del $\mathrm{O}_{2}$ para 
genera oxígeno singlete $\left({ }^{1} \mathrm{O}_{2}\right)$, especie muy reactiva que participa en la generación de estrés oxidativo en sistemas biológicos.

\subsubsection{Transferencia de electrones}

En el estado excitado, las moléculas sufren cambios en sus propiedades fisicoquímicas respecto al estado basal, en particular su acidez y potencial redox. Cuando una especie química se oxida o reduce, por reacción con otra especie electrónicamente excitada, se dice que la transferencia de electrones es fotosensibilizada. Esta transferencia de electrones consiste en una reacción en la cual un electrón es cedido ( o aceptado) por una molécula excitada hacia (o desde) un aceptor en su estado fundamental, de acuerdo con los respectivos potenciales redox de los dos estados involucrados. Si la transferencia de electrones se produce entre dos moléculas sin carga se produce un par de iones radicales. La molécula electrónicamente excitada puede ser tanto de tipo "dadora" como "aceptora" de electrones:

$$
\begin{aligned}
& \text { Sens }^{*}+\mathrm{A} \longrightarrow \text { Sens }^{\circ+}+\mathrm{A}^{\circ-} \\
& \text { Sens }^{*}+\mathrm{A} \longrightarrow \text { Sens }^{\circ-}+\mathrm{A}^{\bullet+}
\end{aligned}
$$

Los radicales formados pueden posteriormente sufrir diferentes reacciones que dependerán de sus propiedades, de la presencia de otros compuestos en el medio y de diversas condiciones experimentales, como son la temperatura, el $\mathrm{pH}$ y las características del solvente.

\subsection{Oxidaciones fotosensibilizadas}

Las fotooxidaciones son un conjunto de procesos fotoinducidos de gran importancia en sistemas biológicos. Si, además, la fotooxidación ocurre en una molécula como resultado de la absorción de radiación por otra (sensibilizador), el proceso es una oxidación fotosensibilizada. Estos procesos pueden ocurrir a través de un conjunto de mecanismos que pueden involucrar tanto fenómenos de transferencia de energía como de electrones. El oxígeno que participa en las oxidaciones puede estar en su estado triplete basal o en un estado excitado singlete. ${ }^{8}$

La clasificación más aceptada de los mecanismos de las oxidaciones fotosensibilizadas es, muy probablemente, la propuesta por Foote (Esquema 1.4).9 El primer paso, como en todo proceso fotosensibilizado, implica la absorción de la 
radiación por un sensibilizador (Sens) generando, de esta manera, un estado excitado del sensibilizador (Sens*), el cual puede ser singlete o triplete. El sensibilizador electrónicamente excitado puede reaccionar con el sustrato o el solvente (mecanismos Tipo I) o con el $\mathrm{O}_{2}$ (mecanismos Tipo II).

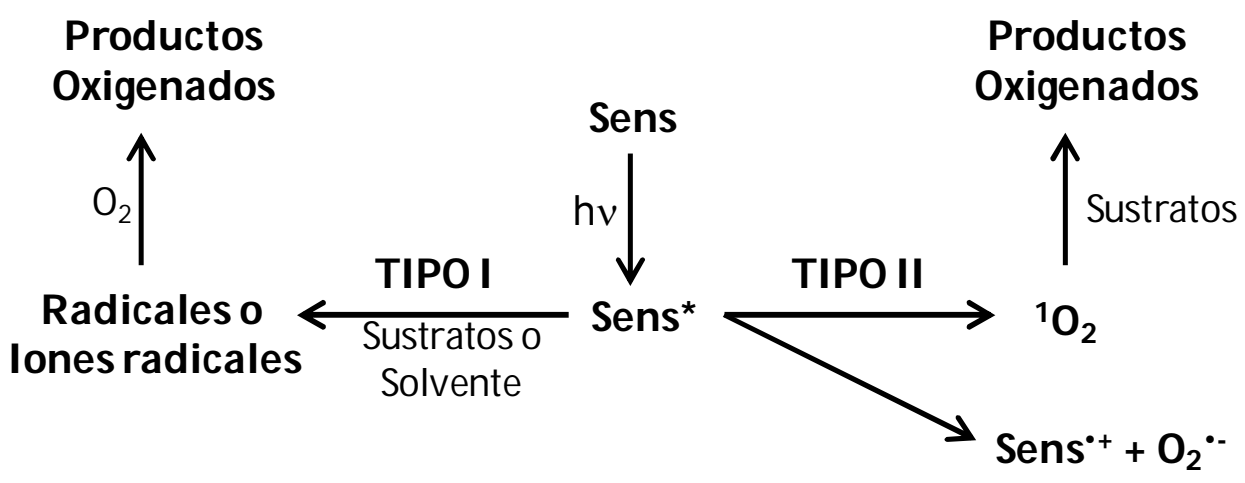

Esquema 1.4 Oxidaciones fotosensibilizadas de Tipo Iy Tipo II.9

\subsubsection{Mecanismos Tipo I}

En los mecanismos Tipo I, el sensibilizador en estado excitado reacciona con el sustrato o el solvente, a través de un proceso de transferencia de un electrón, generando el radical anión y catión de las distintas especies (reacciones 1-2), o de abstracción de un átomo de hidrógeno (reacciones 3-4), generando radicales. Estos radicales reaccionan con el $\mathrm{O}_{2}$ disuelto produciendo $\mathrm{O}_{2}{ }^{\bullet-(}$ (reacciones 5-6), el cual, posteriormente, reacciona con el sustrato o su radical para formar productos oxigenados $\left(\mathrm{A}_{(\mathrm{ox})}\right)$ (reacciones 7-8) (Esquema 1.5).

$$
\begin{aligned}
& \text { Sens* }+\mathrm{A} \longrightarrow \text { Sens }{ }^{\circ-}+\mathrm{A}^{\cdot+} \\
& \text { Sens }^{*}+A \longrightarrow \text { Sens }^{\circ+}+A^{\bullet-} \\
& \text { Sens }^{*}+\mathrm{A} \longrightarrow \operatorname{SensH}^{\circ}+\mathrm{A}(-\mathrm{H})^{\circ} \\
& \text { Sens }^{*}+\mathrm{A} \longrightarrow \operatorname{Sens}(-\mathrm{H})^{\bullet}+\mathrm{AH}^{\bullet} \\
& \text { Sens }{ }^{\circ-}+\mathrm{O}_{2} \longrightarrow \text { Sens }+\mathrm{O}_{2}{ }^{-\cdot} \\
& \mathrm{A}^{--}+\mathrm{O}_{2} \longrightarrow \mathrm{A}+\mathrm{O}_{2}{ }^{\circ-} \\
& \mathrm{A} \stackrel{\mathrm{O}_{2}{ }^{\bullet-}}{\longrightarrow} \mathrm{A}_{(\mathrm{ox})} \\
& A^{\cdot+} \stackrel{\mathrm{O}_{2}-/ \mathrm{O}_{2} / \mathrm{H}_{2} \mathrm{O}}{\longrightarrow} A_{(\mathrm{ox})}
\end{aligned}
$$




\subsubsection{Mecanismos Tipo II}

Los mecanismos de Tipo II involucran la producción de ${ }^{1} \mathrm{O}_{2}$ por procesos de transferencia de energía. $\mathrm{El}_{2}$ en estado basal triplete interacciona con el estado triplete excitado del sensibilizador dando lugar a la desactivación de Sens* y la formación de ${ }^{1} \mathrm{O}_{2}$. Luego, esta última especie, altamente reactiva, oxida al sustrato (A) generando productos oxigenados $\left(\mathrm{A}_{(\mathrm{ox})}\right)$ (reacciones 11-12) (Esquema 1.6).

Sin embargo, también puede existir una transferencia de electrones desde el sensibilizador hacia el $\mathrm{O}_{2}$, formando el radical catión del sensibilizador (Sens*+) y $\mathrm{O}_{2}{ }^{*}$. Esta especie reactiva puede, posteriormente, oxidar al sustrato (reacciones 13-14, Esquema 1.6). Si bien en este mecanismo la oxidación del sustrato se produce por reacción con $\mathrm{O}_{2} *$, al igual que en la reacción 7 (Esquema 1.5) de los mecanismos Tipo I, el mismo entra en la categoría de mecanismos de Tipo II, de acuerdo a la clasificación de Foote (Esquema 1.4), debido a que el sensibilizador excitado reacciona directamente con el $\mathrm{O}_{2}$.

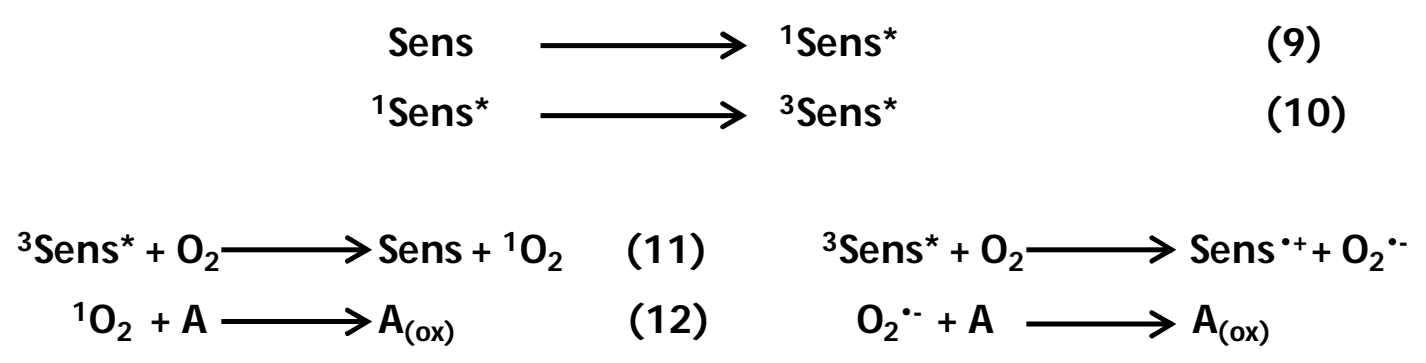

\section{Esquema 1.6 Reacciones posible en fotooxidación de Tipo II}

\subsection{Referencias}

1. J. R. Lakowicz, Principles of Fluorescence Spectroscopy, Springer, 3rd edn., 2006.

2. V. A. Gilbert and J. Baggott, Essentials of Molecular Photochemistry, Blackwell Scientific Publications, Oxford 1991.

3. N.J. Turro, Modern Molecular Photochemistry, Univeristy Science Books, 1991.

4. S. E. Braslavsky, Glossary of terms used in photochemistry, 3rd edition, Pure and Applied Chemistry, 2007, 79, 293-465.

5. J. C. Scaiano, Handbook of Organic Photochemistry, CRC Press, 1989.

6. C. E. Wayne and R. P. Wayne, Photochemistry, Oxford Univerity Press, Oxford, 1996.

7. A. M. Braun, M. T. Maurette and E. Oliveros, Photochemical Technology, John Wiley \& Sons, Chichester, 1991.

8. P. Klán and J. Wirz, Photochemistry of Organic Compounds: From Concepts to Practice, John Wiley \& Sons Ltd., Chichester, 2009.

9. C. S. Foote, Definition of Type I and Type II photosensitized oxidation, Photochemistry and Photobiology, 1991, 54, 659-659. 
12 Capítulo 1 


\section{Capítulo 2}

\section{Propiedades químicas y bioquímicas de pteridinas}

Las pteridinas son una familia de compuestos orgánicos heterocíclicos muy distribuidos en la naturaleza. Aunque se encuentran presentes en muy pequeñas cantidades en los seres vivos, son esenciales en los mamíferos ya que desempeñan diversas funciones. Los primeros trabajos científicos relacionados con las pteridinas, se llevaron a cabo en el año 1889. En estos trabajos, se manifiestan los primeros intentos por aislar algunos pigmentos de diferentes clases de mariposas.1-3 Más tarde, se elucidó la estructura molecular de estos pigmentos, planteándolos como derivados del heterociclo pirazina [2,3-d] pirimidina ${ }^{4}, 5$ al que, posteriormente, se lo denominó pteridina. 6

\subsection{Estructura química}

Las pteridinas son moléculas que poseen un doble anillo de diez átomos, cuatro de los cuales son átomos de nitrógeno. Por tal motivo, pueden definirse como tetra-azonaftalenos. En la Figura 2.1 se observa la estructura heterocíclica común a todas las pteridinas con la correspondiente numeración. El anillo I corresponde a la estructura de la pirimidina, mientras que el anillo II a la pirazina. Asimismo, en la Figura 2.1 se muestran las dos estructuras químicas de las cuales se derivan las "pteridinas naturales" presentes en los seres vivos: 2-aminopterinidin-4(3H)-ona o pterina y pteridin-2,4(1H, 3H)-diona o lumazina.

Los derivados pterínicos se encuentran normalmente sustituidos en la posición 6 del doble anillo. Según el tamaño de este sustituyente las pterinas pueden dividirse en dos grupos:

$\checkmark$ pterinas no conjugadas, cuyos sustituyentes poseen una corta cadena hidrocarbonada o un solo átomo de carbono,

$\checkmark$ pterinas conjugadas, cuyos sustituyentes son de mayor peso molecular e incluyen una molécula de ácido p-aminobenzoico (PABA); un ejemplo es el ácido fólico (PteGlu) que además de PABA posee una molécula de ácido glutámico unidas por una unión peptídica. 
<smiles></smiles>

Pteridina<smiles>Nc1nc2nccnc2c(=O)[nH]1</smiles>

Figura 2.1 Estructura química básica de las pteridinas con sus dos anillos típicos: pirimidínico (I) y pirazínico (II). Estructuras de las pteridinas naturales lumazina y pterina.

En la naturaleza las pterinas pueden encontrarse en diferentes estados de oxidación, donde el anillo pirazina es el que está parcial o totalmente hidrogenado. Las pterinas reducidas más comúnmente encontradas en sistemas biológicos son las 7,8dihidropterinas y las 5,6,7,8-tetrahidropterinas (Figura 2.2).<smiles>[R]c1cnc2c(=O)[nH]c(N)nc2n1</smiles>

pterina aromática<smiles>[R]C1=Nc2c(nc(N)[nH]c2=O)N[Z]1([H])[H]</smiles>

7,8-dihidropterina<smiles>[R]C1CNc2nc(N)[nH]c(=O)c2N1</smiles>

5,6,7,8-tetrahidropterina

Figura 2.2 Estructura química de pterina aromática, 7,8-dihidropterina y 5,6,7,8tetrahidropterina.

En la Figura 2.3 se presentan los distintos isómeros estructurales que es posible encontrar para las dihidropterinas.7 De estos cinco isómeros, solo los 7,8-dihidro han sido sintetizados y caracterizados, ya que son los termodinámicamente más estables gracias a su distribución electrónica. ${ }^{8}$ 
<smiles>[R]C1C=Nc2nc(N)[nH]c(=O)c2N1</smiles>

5,6-dihidropterina<smiles>[R]C1=CNc2nc(N)[nH]c(=O)c2N1</smiles>

5,8-dihidropterina<smiles>[R]C1=Nc2c(nc(N)[nH]c2=O)NC1[2H]</smiles>

7,8-dihidropterina<smiles>[R]C1CN=c2nc(N)[nH]c(=O)c2=N1</smiles>

6,7-dihidropterina<smiles>[R]C1CNC2=NC(N)=NC(=O)C2=N1</smiles>

6,7,8-dihidropterina

Figura 2.3 Isómeros estructurales de dihidropterinas.

En este trabajo de tesis se estudiaron particularmente dos pterinas oxidadas, biopterina (Bip) y neopterina (Nep) y sus respectivos dihidroderivados, 7,8dihidrobiopterina ( $\mathrm{H}_{2} \mathrm{Bip}$ ) y 7,8-dihidroneopterina $\left(\mathrm{H}_{2} \mathrm{Nep}\right)$. Además, se utilizaron otros derivados pterínicos cuyas estructuras se presentan en la Tabla 2.1.<smiles>[R]c1cnc2nc(N)[nH]c(=O)c2n1</smiles><smiles>[R]C1=Nc2c(nc(N)[nH]c2=O)NC1</smiles>

\begin{tabular}{ccc}
\hline $\mathbf{R}$ & Pterina oxidada & Dihidroderiva do \\
\hline$-(\mathrm{CHOH})_{2}-\mathrm{CH}_{2} \mathrm{OH}$ & neopterina (Nep) & 7,8-dihidroneopterina $\left(\mathrm{H}_{2} \mathrm{Nep}\right)$ \\
$-(\mathrm{CHOH})_{2}-\mathrm{CH}_{3}$ & biopterina (Bip) & 7,8-dihidrobiopterina $\left(\mathrm{H}_{2} \mathrm{Bip}\right)$ \\
$-\mathrm{CHO}$ & 6-formilpterina (Fop) & 6-formil-7,8-dihidropterina $\left(\mathrm{H}_{2} \mathrm{Fop}\right)$ \\
$-\mathrm{CH}_{3}$ & 6-metilpterina (Mep) & - \\
$-\mathrm{CH}_{2} \mathrm{OH}$ & 6-hidroximetilpterina (Hmp) & - \\
$-\mathrm{COOH}$ & 6-carboxipterina (Cap) & - \\
$-\mathrm{H}$ & pterina (Ptr) & - \\
\hline
\end{tabular}

7,8-dihidroxantopterina ( $\left.\mathrm{H}_{2} \mathrm{Xap}\right)$<smiles>Nc1nc2c(c(=O)[nH]1)NC(=O)CN2</smiles>

Tabla 2.1 Nomenclatura y estructura química de pterinas oxidadas y 7,8-dihidropterinas utilizadas en este trabajo de tesis.

\subsection{Propiedades ácido-base y espectros de absorción}

En la Figura 2.4 se muestra el equilibrio ácido-base más importante de las pterinas en solución acuosa, considerando los valores de $\mathrm{pH}$ presentes en los sistemas 
biológicos. La forma ácida (amida) de este equilibrio corresponde a la forma neutra, mientras que la forma alcalina (fenolato) posee una carga negativa. Este equilibrio ya ha sido estudiado en solución acuosa, habiéndose publicado valores de $\mathrm{pK}_{\mathrm{a}}$ de alrededor de 8 para las pterinas oxidadas, 9-13 y de alrededor de 10 para las dihidropterinas.7, 9, 14, 15 Este comportamiento general está afectado por la presencia de sustituyentes adicionales en el doble anillo pterínico, los cuales modifican, en mayor o menor medida, los valores de $\mathrm{pK}_{\mathrm{a}}$ y la carga neta de las moléculas. Los valores de $\mathrm{pK}_{\mathrm{a}}$ de los compuestos utilizados en este trabajo de tesis y otros se presentan en la Tabla 2.2.<smiles>[R]c1cnc2nc(N)nc([O-])c2n1</smiles>

forma ácida

forma alcalina

Figura 2.4 Equilibrio ácido-base del anillo pterínico en soluciones acuosas de pH entre 4 y 11.

\begin{tabular}{cccccc}
\hline Compuesto & $\mathbf{p K}_{\mathbf{a}}$ & Ref. & Compuesto & $\mathbf{p K}_{\mathbf{a}}$ & Ref. \\
\hline Bip & 8,1 & 13 & $\mathbf{H}_{2}$ Bip & 10,85 & 14 \\
\hline Nep & 8,0 & 13 & $\mathbf{H}_{2}$ Nep & 10,62 & 16 \\
\hline Fop & 7,33 & 11 & $\mathbf{H}_{2}$ Fop & 9,68 & 16 \\
\hline Mep & 8,3 & 17 & $\mathbf{H}_{2}$ Mep & 10,85 & 7 \\
\hline Ptr & 7,9 & 10 & $\mathbf{H}_{2}$ Xap & 9,91 & 16 \\
\hline Hmp & 8,1 & 18 & & & \\
\hline Cap & 7,9 & 19 & & & \\
\hline
\end{tabular}

Tabla 2.2 Valores de $\mathrm{pK}_{\mathrm{a}}$ del equilibrio amida-fenolato de distintas pterinas.

Cuando el pH es inferior a 3, existen otros equilibrios ácido-base en los que participan otros grupos funcionales de la estructura pterínica, como son los nitrógenos anulares o el grupo amino de la posición 2 del anillo. A medida que disminuye el pH, estos grupos comienzan a protonarse, apareciendo las correspondientes formas catiónicas con carga $+1,+2$ y +3 . Todos estos equilibrios presentan valores de $\mathrm{pK}_{\mathrm{a}}$ inferiores a $4 .{ }^{9}$

Sin embargo, en las condiciones de $\mathrm{pH}$ utilizadas en este trabajo de tesis $\mathrm{pH}=4$ 11), el único equilibrio que se detecta es aquel entre el grupo amida y el fenolato, mencionado anteriormente (Figura 2.4). Es importante mencionar que las constantes de 
este equilibrio entre las dos formas ácido-base de los estados excitados singletes $\left(\mathrm{k}_{\mathrm{a}}{ }^{*}\right)$ fue estudiado para ciertos derivados pterínicos encontrando valores muy similares a los obtenidos para el equilibrio en estado basal.13, 19, 20 Es importante tener en cuenta este equilibrio debido a que, según estudios publicados para otros derivados pterínicos, tanto el comportamiento fotoquímico como fotofísico de las diferentes formas ácidobase de cada compuesto presentan variaciones significativas. ${ }^{21-23}$

Las características espectrales de los derivados pterínicos, por su parte, dependen de diferentes factores, tales como el estado de oxidación del anillo pirazina, la naturaleza química de los sustituyentes unidos al doble anillo pterínico y las condiciones de $\mathrm{pH}$ del medio. El espectro de absorción de la mayoría de la pterinas aromáticas no conjugadas presenta dos bandas principales de absorción, una banda con máximo entre 220 y $290 \mathrm{~nm}$ y la otra, de menor energía, con un máximo cercano a $350 \mathrm{~nm} .{ }^{17}, 24 \mathrm{La}$ banda correspondiente a la transición $\mathrm{S}_{0} \rightarrow \mathrm{S}_{2}$ de la forma ácida presenta un máximo de absorción a $280 \mathrm{~nm}$ aproximadamente, mientras que para la forma alcalina se encuentra en $255 \mathrm{~nm}$. La intensidad de ambas transiciones es menor para la forma ácida que para la alcalina. Por otra parte, la banda de menor energía correspondiente a la transición $\mathrm{S}_{0} \rightarrow \mathrm{S}_{1}$ de la forma ácida presenta un corrimiento hacia el azul $\left(\lambda_{\max } \sim 340-350 \mathrm{~nm}\right) \mathrm{si}$ se la compara con la forma básica $\left(\lambda_{\max } \sim 355-370 \mathrm{~nm}\right.$ ). En la Figura 2.5 se presentan los espectros de absorción en solución acuosa de las formas ácidas y básicas de las cuatro pterinas oxidadas que aparecerán con mayor frecuencia a lo largo de este trabajo de tesis: Bip, Nep, 6-formilpterina (Fop) y 6-carboxipterina (Cap). Cabe destacar que el espectro de Fop posee una forma particular, y distinta al de los otros derivados pterínicos. Esto se debe a que tiene como sustituyente en el C6, un grupo carbonilo cuyo enlace modifica los niveles de energía del doble anillo y genera la aparición de una tercera banda entre las dos descriptas anteriormente. 

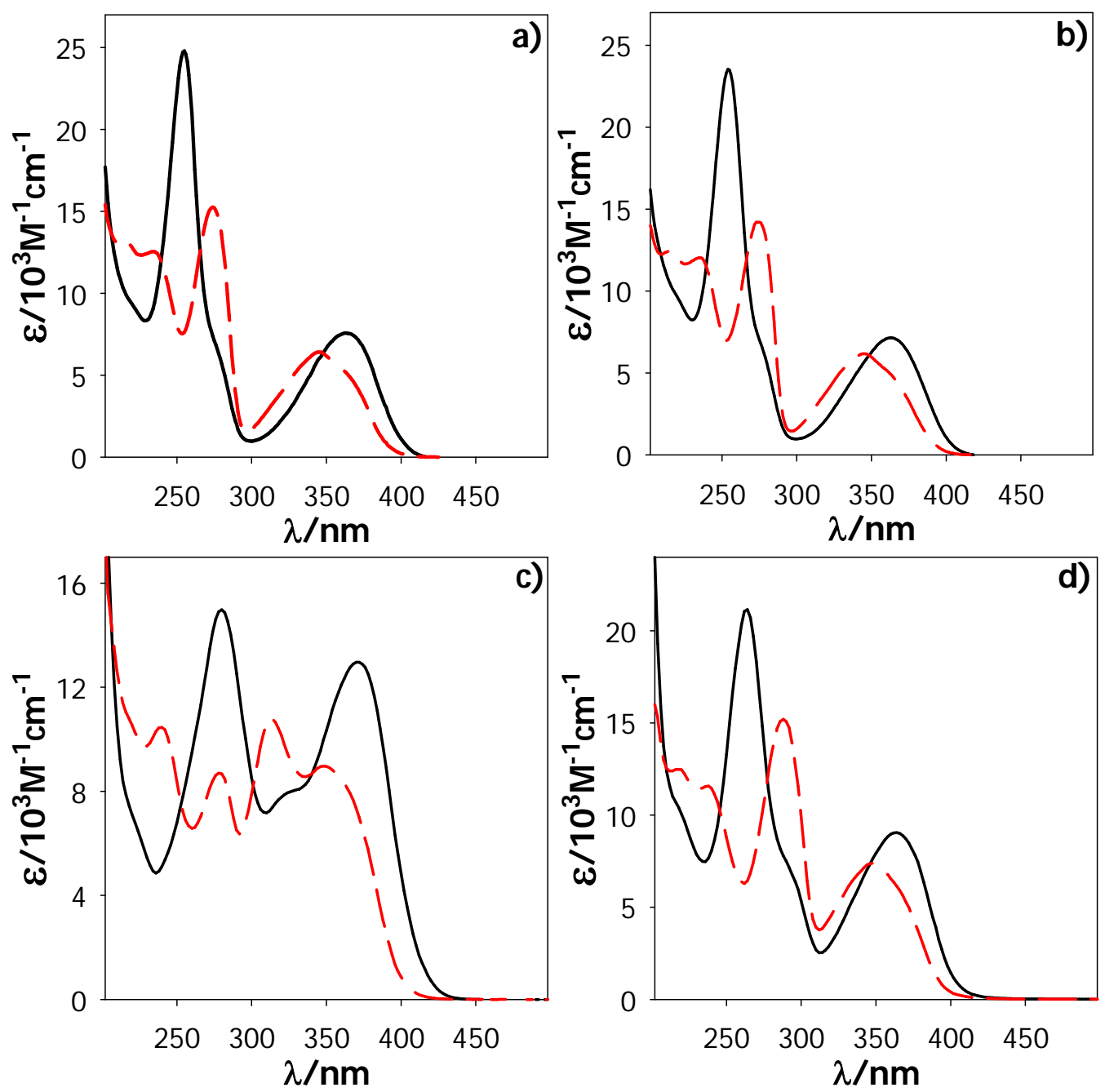

Figura 2.5 Espectros de absorción de pterinas aromáticas en solución acuosa. Formas ácida y alcalina en color rojo y negro, respectivamente. a) Bip, b) Nep, c) Fop, d) Cap.

Con respecto a los espectros de absorción de las dihidropterinas, estos son muy variables y dependientes de la naturaleza química del sustituyente en el C6. En general, poseen tres bandas: la banda correspondiente a la transición $\mathrm{S}_{0} \rightarrow \mathrm{S}_{3}$ con un $\lambda_{\max } \approx 230$ $\mathrm{nm}$, la banda correspondiente a transición $\mathrm{S}_{0} \rightarrow \mathrm{S}_{2}$ con $\lambda_{\max }$ entre 270 y $280 \mathrm{~nm}$, y la banda de menor energía, con $\lambda$ máximo entre $310 \mathrm{~nm}$ y $330 \mathrm{~nm}$. Aquellos derivados que poseen en el Ca del sustituyente de la posición 6, un grupo carbonilo, presentan una banda de gran intensidad en la región visible del espectro. Esto se atribuye a una interacción mesomérica entre el grupo amino de posición 2 que actúa como dador de electrones y el grupo carbonilo del sustituyente de posición 6, quien actúa como aceptor de electrones. Los dos dihidroderivados estudiados en este trabajo de tesis, $\mathrm{H}_{2} \mathrm{Bip}$ y $\mathrm{H}_{2} \mathrm{Nep}$, presentan espectros muy similares, como se observa en la Figura 2.6. 

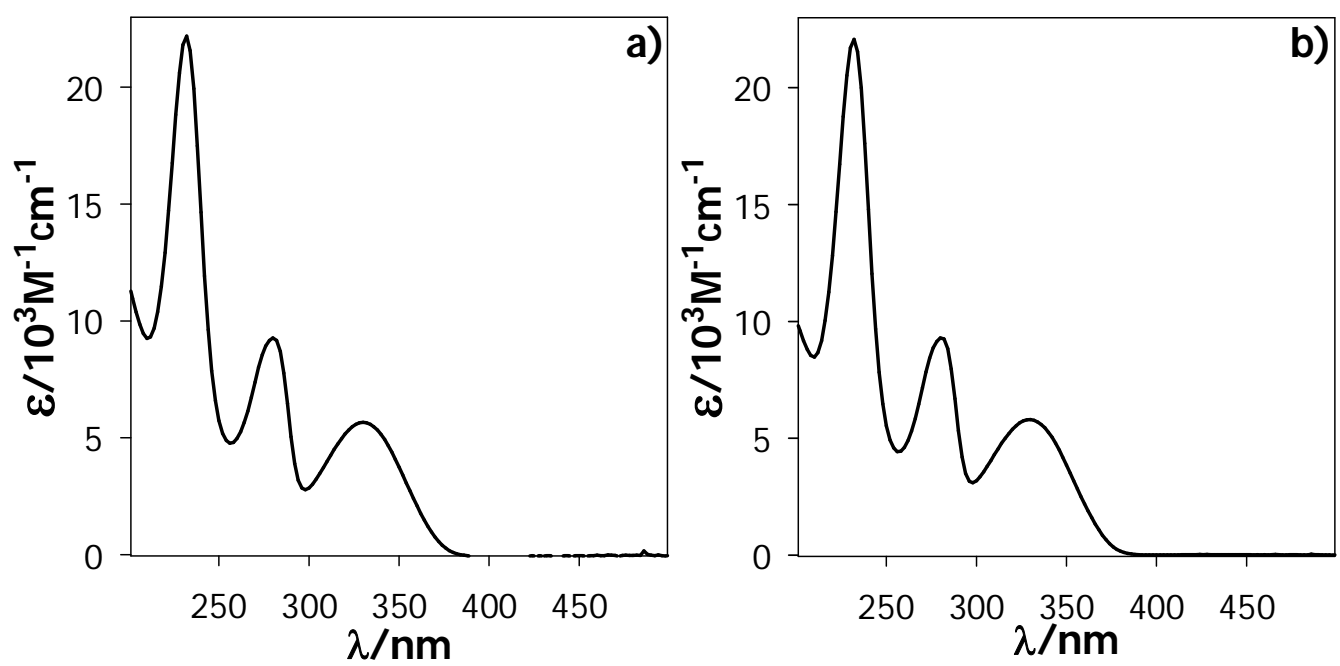

Figura 2.6 Espectros de absorción de las formas ácidas de las 7,8-dihidropterinas en solución acuosa a pH $\sim 7$. a) $\mathrm{H}_{2} \mathrm{Bip}$, b) $\mathrm{H}_{2} \mathrm{Nep}$.

\subsection{Reactividad química de pterinas}

En general, las soluciones acuosas de las pterinas aromáticas son estables al aire, aunque esta propiedad varía ligeramente con el sustituyente presente en la posición 6 . Por ejemplo las soluciones de pterina (Ptr), Cap y 6-metilpterina (Mep) duran varias semanas sin descomponerse, mientras que las soluciones de Bip, Nep y Fop, duran solo algunos días. Por el contrario, las soluciones de las dihidropterinas presentan una inestabilidad significativa habiéndose realizado numerosos estudios para investigar la oxidación que sufren en presencia de $\mathrm{O}_{2}$. En el caso de los dihidroderivados estudiados en este trabajo de tesis, $\mathrm{H}_{2}$ Bip y $\mathrm{H}_{2} \mathrm{Nep}$, su reacción con $\mathrm{O}_{2}$ lleva a la formación de $\mathrm{H}_{2}$ Xap como principal producto de oxidación (> 80 \%) y $\mathrm{H}_{2}$ Fop como producto minoritario (Esquema 2.1). Estos compuestos poseen una constante aparente de velocidad de reacción con $\mathrm{O}_{2}\left(\mathrm{k}_{\mathrm{ap}}\right)$ a $25^{\circ} \mathrm{C}$ de $0,0004 \pm 0,0002$ y 0,012 $\pm 0,003 \mathrm{~h}^{-1}$ para $\mathrm{H}_{2}$ Bip y $\mathrm{H}_{2} \mathrm{Nep}$, respectivamente. ${ }^{16}$ Teniendo en cuenta estos valores de $\mathrm{k}_{\mathrm{ap}} \mathrm{y}$ los correspondientes tiempos de vida media ( $\mathrm{t}_{1 / 2}=1700$ y $60 \mathrm{~h}$, para $\mathrm{H}_{2}$ Bip y $\mathrm{H}_{2} \mathrm{Nep}$, respectivamente), se pudo trabajar sin problemas con las soluciones preparadas en el día, en el tiempo que duraron los experimentos de este trabajo de tesis ( $<7 \mathrm{~h})$. 
<smiles>[R]C1=Nc2c(nc(N)[nH]c2=O)NC1</smiles><smiles>[Y17][Y12]1([H])CC(=O)Nc2c1nc(N)[nH]c2=O</smiles>

$\mathrm{H}_{2} \mathrm{Fop}$

\section{Esquema 2.1 Oxidación de $\mathrm{H}_{2}$ Bip y $\mathrm{H}_{2}$ Nep por $\mathrm{O}_{2}$ en soluciones acuosas neutras.}

Además de la reacción con $\mathrm{O}_{2}$, los dihidroderivados se oxidan fácilmente frente a especies reactivas de oxígeno como $\mathrm{H}_{2} \mathrm{O}_{2}$ y ${ }^{1} \mathrm{O}_{2}$. Cuando $\mathrm{H}_{2} \mathrm{Bip}$ o $\mathrm{H}_{2} \mathrm{Nep}$ se enfrentan con concentraciones apreciables de $\mathrm{H}_{2} \mathrm{O}_{2}$, sufren una rotura del sustituyente y oxidación del átomo de $\mathrm{C}$ en posición 6, para generar como producto principal $\mathrm{H}_{2} \mathrm{Xap}(<90 \%)$ (Esquema 2.2). Estos compuestos presentan constantes bimoleculares de reacción $(\mathrm{k}) \mathrm{a}$ $37^{\circ} \mathrm{C}$ en soluciones equilibradas en aire a $\mathrm{pH} \approx 7$, de $0,027 \pm 0,002$ y $0,037 \pm 0,004 \mathrm{M}^{-1} \mathrm{~S}^{-1}$ para $\mathrm{H}_{2}$ Bip y $\mathrm{H}_{2} \mathrm{Nep}$, respectivamente. ${ }^{25}$<smiles>[R]C1=Nc2c(nc(N)[nH]c2=O)NC1C(C)O</smiles>

\section{Esquema 2.2 Oxidación de $\mathrm{H}_{2} \mathrm{Bip}$ y $\mathrm{H}_{2} \mathrm{Nep}$ por $\mathrm{H}_{2} \mathrm{O}_{2}$ en soluciones acuosas neutras.}

Con respecto al ${ }^{1} \mathrm{O}_{2}$, ambas pterinas reaccionan con altas constantes de quenching total $\left(\mathrm{k}_{\mathrm{t}}\right)$, dada principalmente por una alta eficiencia de reacción química con esta especie reactiva (elevada constante de quenching químico, $\mathrm{k}_{\mathrm{r}}$ ). $\mathrm{H}_{2}$ Bip reacciona con ${ }^{1} \mathrm{O}_{2}$ generando la oxidación del anillo pirazina para dar Bip ( $\approx 45 \%$ ) y $\mathrm{H}_{2} \mathrm{O}_{2}$ en menor cantidad, y otros productos no identificados. Por otro lado, $\mathrm{H}_{2} \mathrm{Nep}$ además de sufrir oxidación del anillo para generar Nep ( $\approx 25 \%$ ) y otros productos no identificados, sufre oxidación del Cen posición 6 para producir $\mathrm{H}_{2}$ Xap (Esquema 2.3) 
a)

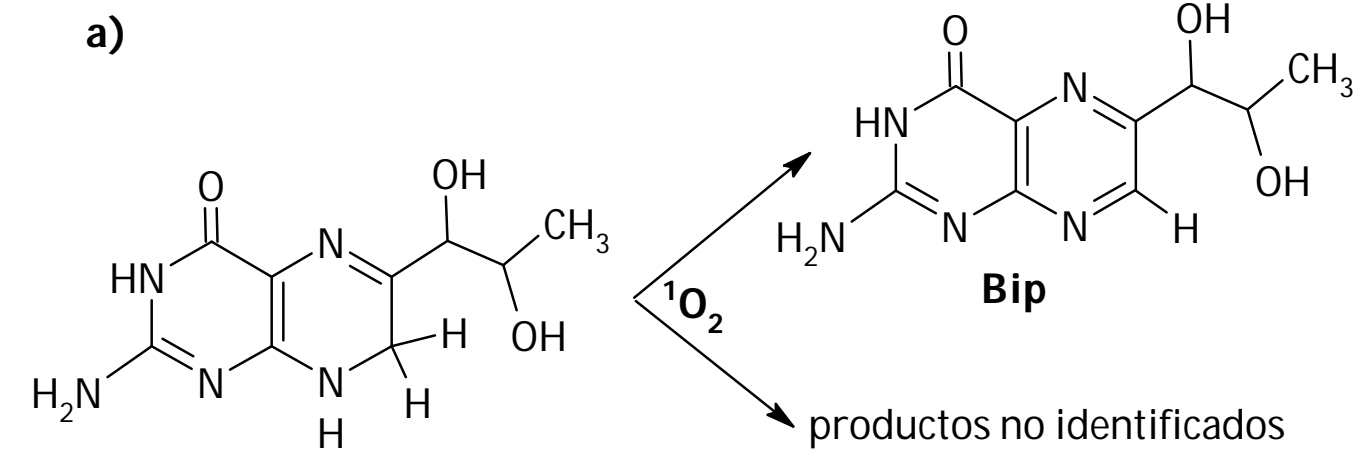
$\mathrm{H}_{2}$ Bip

b)<smiles></smiles>

Esquema 2.3 Oxidación de $\mathrm{H}_{2} \mathrm{Bip}$ (a) y $\mathrm{H}_{2} \mathrm{Nep}$ (b) por ${ }^{1} \mathrm{O}_{2}$ en soluciones acuosas neutras.

\subsection{Bioquímica de biopterina y sus derivados reducidos}

Aunque las pterinas se encuentran en muy pequeñas cantidades en los organismos vivos, participan en importantes reacciones bioquímicas. 5,6,7,8Tetrahidrobiopterina ( $\mathrm{H}_{4} \mathrm{Bip}$, Figura 2.2) es una molécula que actúa como cofactor de varias enzimas. De esta manera, está involucrada en diferentes proceso biológicos de gran importancia para los seres vivos, tales como funciones del sistema nervioso, ${ }^{26,}, 27$ proliferación celular y homeostasis vascular. 28,29

Por un lado, $\mathrm{H}_{4} \mathrm{Bip}$ es cofactor de las tres isoformas de la enzima óxido nítrico sintasa (NOS): endotelial (eNOS), neuronal (nNOS) e inducible (iNOS). Estas enzimas catalizan la formación de óxido nítrico (NO*) a partir de la oxidación de arginina. Además de funcionar como dador de electrones, $\mathrm{H}_{4} \mathrm{Bip}$ estabiliza la estructura dimérica de las NOSs, que es la forma catalíticamente activa, ${ }^{30,31}$ aumenta la afinidad de la enzima por el sustrato ${ }^{32}$ y aumenta la velocidad de consumo de $\mathrm{NADPH}^{33}$ entre otras funciones. 
El NO• formado participa como intermediario en diversos procesos, como la vasodilatación y la regulación del tono vascular normal, la inhibición de la agregación plaquetaria, transmisión neuronal y citostasis. ${ }^{34} \mathrm{H}_{2}$ Bip también se une a la enzima con la misma afinidad y en la eNOS genera desacoplamiento de la enzima que conlleva a una producción de anión superóxido $\left(\mathrm{O}_{2}{ }^{*}\right)$ con la consecuente disfunción endotelial. ${ }^{35}$

Asimismo, este tetrahidroderivado actúa como cofactor de las hidroxilasas de aminoácidos aromáticos. Existen tres tipos de hidroxilasas:

$\checkmark$ Fenilalanina hidroxilasa (PAH), que convierte la fenilalanina en tirosina, reacción muy importante en el catabolismo de este aminoácido y, primer paso de la biosíntesis de melanina, el pigmento natural que protege a los seres humanos de la radiación UV.

$\checkmark$ Tirosina hidroxilasa (TH), que cataliza la conversión de tirosina a 3,4-hidroxiL-fenilalanina (L-DOPA), primer paso en la biosíntesis de catecolaminas (dopamina, noradrenalina y adrenalina).

$\checkmark$ Triptofano hidroxilasa (TPH), necesaria para la síntesis de serotonina y melatonina a partir del triptófano.

Estas tres enzimas son estructuralmente muy similares entre sí. Para catalizar la reacción requieren $\mathrm{H}_{4} \mathrm{Bip}, \mathrm{O}_{2}$ y hierro. El sustrato se hidroxila y el $\mathrm{O}_{2}$ se reduce a $\mathrm{H}_{2} \mathrm{O}$. La $\mathrm{H}_{4} \mathrm{Bip}$ proporciona los electrones para reducir al $\mathrm{O}_{2}$ y forma un intermediario peroxi $\mathrm{H}_{4} \mathrm{Bip}$, el cual cede un átomo de oxígeno a la molécula que se hidroxilará mientras que el átomo de oxígeno remanente forma un hidroxi-derivado de $\mathrm{H}_{4} \mathrm{Bip}$. Este hidroxi-derivado pasa a una forma quinoide parcialmente reducida ( $\mathrm{qH}_{2} \mathrm{Bip}$ ). $\mathrm{La} \mathrm{qH}_{2} \mathrm{Bip}$ por medio de una serie de pasos catalizados por enzimas es reducida totalmente para dar nuevamente $\mathrm{H}_{4} \mathrm{Bip}$ (Esquema 2.4). Además, $\mathrm{H}_{4}$ Bip tiene una segunda función en estas enzimas que es la de reducir el hierro en el sitio activo de la enzima de Fe (III) a Fe (II). ${ }^{36}$ 
<smiles>NC(Cc1ccccc1)C(=O)O</smiles><smiles>NC(Cc1ccc(O)cc1)C(=O)O</smiles><smiles>N[C@@H](Cc1ccc(O)cc1)C(=O)O</smiles>

PAH<smiles>C1CCCCC1</smiles>

tirosina

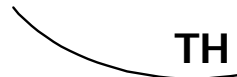

HO

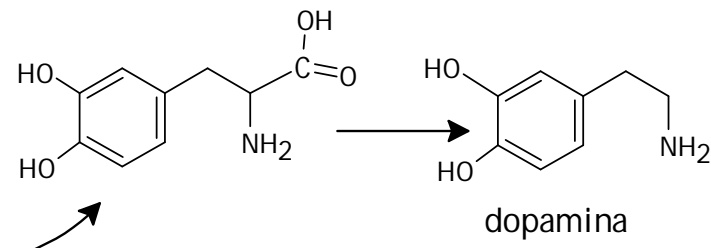<smiles>NC(Cc1c[nH]c2ccccc12)C(=O)O</smiles><smiles>Cc1c[nH]c2ccc(O)cc12</smiles>
triptofano

$\mathrm{O}_{2}$

TPH<smiles>Oc1ccccc1</smiles><smiles>CCCCC(N)C(=O)O</smiles>
serotonina<smiles>CC(O)C(O)C1CNc2nc(N)[nH]c(=O)c2N1</smiles>

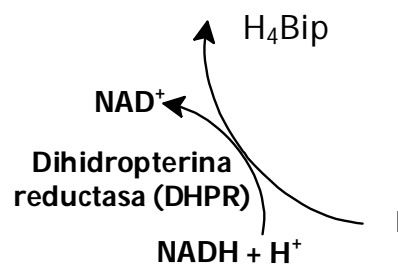<smiles>CC(O)C(O)C1CNC2=NC(N)NC(=O)C2(O)N1</smiles>

$4 \mathrm{a}-\mathrm{OH}-\mathrm{H}_{4} \mathrm{Bip}$<smiles>CCN=C1NCCCC1N</smiles>

$\mathrm{qH}_{2} \mathrm{Bip}$

\section{Esquema 2.4 Participación de $\mathrm{H}_{4} B i p$ en las hidroxilasas}

La reacción catalizada por PAH es la primera de la síntesis de melanina que tiene lugar en las células de la piel (melanocitos). Es sabido que la melanogénesis está activada por la radiación UV. La fotooxidación directa de polímeros de melanina por UVB (290 - $320 \mathrm{~nm}$ ) conduce al aumento de la pigmentación de novo. Además, produce un incremento del número de melanocitos y de la actividad de la tirosinasa, ${ }^{37,38}$ enzima que cataliza la etapa limitante de la melanogénesis, al generar L-DOPA a partir de tirosina. Bajo condiciones fisiológicas normales, los melanocitos tienen la capacidad de sintetizar de novo, reciclar y regular la síntesis de $\mathrm{H}_{4} \mathrm{Bip}^{39}, 40$ Sin embargo, cuando existe un déficit de $\mathrm{H}_{4} \mathrm{Bip}$ se genera una patología llamada vitíligo, la cual será tratada en mayor detalle en la siguiente sección. 
Como ya se mencionó, $\mathrm{H}_{4} B i p$ puede reciclarse a partir de la vía de recuperación. Como se muestra en el Esquema 2.5, la sepiapterina (Sep) que se forma por vía no enzimática a partir de 6-lactoil tetrahidropterina, luego pasa a $\mathrm{H}_{2} \mathrm{Bip}$, en una reacción catalizada por la sepiapterina reductasa (SR). Luego, $\mathrm{H}_{2} \mathrm{Bip}$ pasa a $\mathrm{H}_{4} \mathrm{Bip}$, cuando es catalizada por la dihidrofolato reductasa (DFHR). ${ }^{41}$ Cuando, por ejemplo, existe deficiencia en la actividad de SR en cerebro, se acumula $\mathrm{H}_{2}$ Bip produciendo un desacoplamiento de la nNOS. Esto produce un aumento en las concentraciones de $\mathrm{O}_{2} \cdot \mathrm{y}$ peroxinitrito (ONOO*) que resultan neurotóxicas. Además, altas concentraciones de $\mathrm{H}_{2}$ Bip inhiben la producción de catecolaminas y serotonina en el sistema nervioso central. Por otro lado, $\mathrm{H}_{2} \mathrm{Bip}$ es el derivado capaz de circular e ingresar a la célula cuando se administra oralmente el $\mathrm{H}_{4}$ Bip. ${ }^{42,43}$

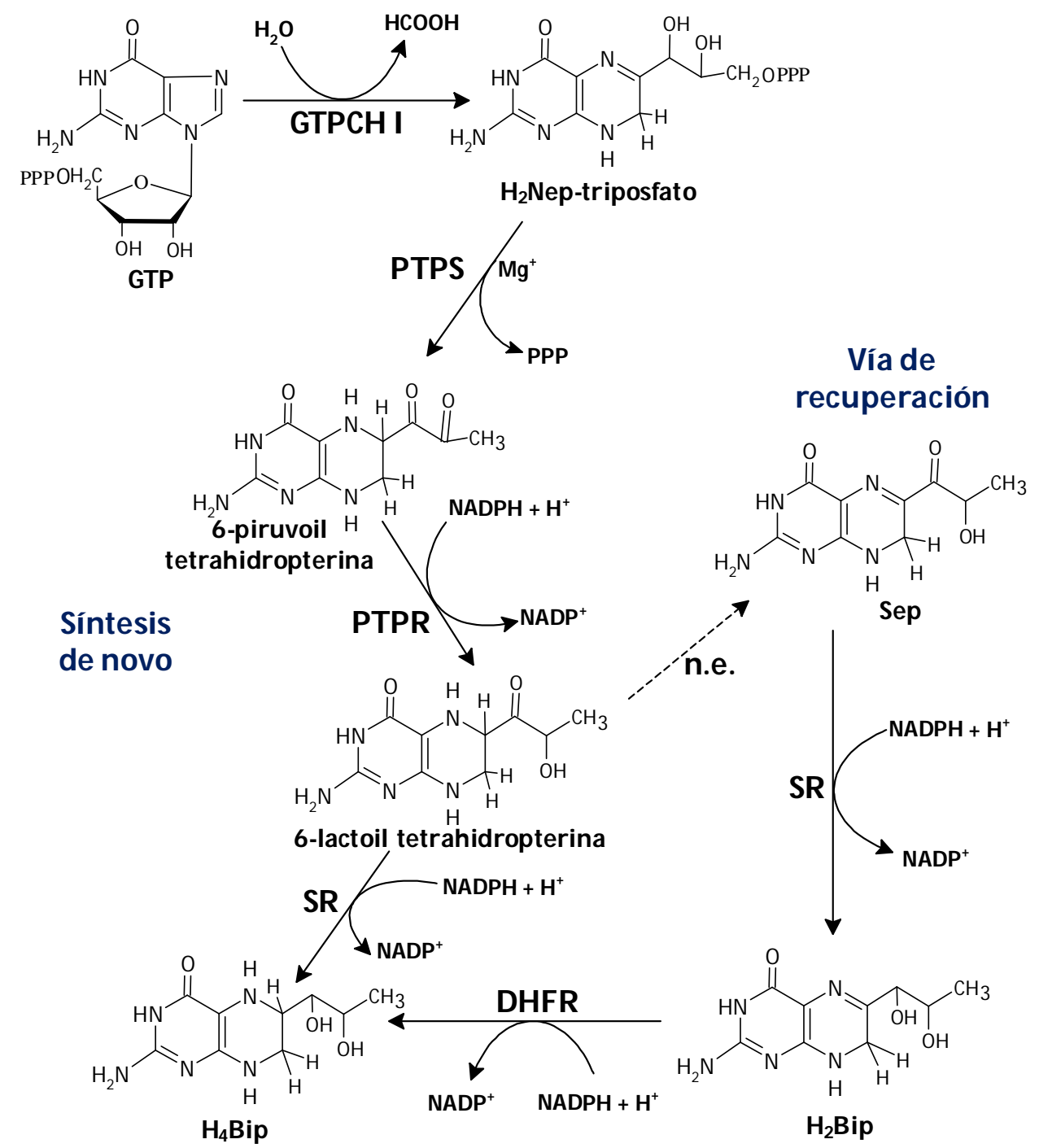

Esquema 2.5 Biosíntesis de $\mathrm{H}_{4} B i p$ : síntesis de novo (izquierda) y vía de recuperación (derecha). GTPCH1: GTP ciclohidroxilasa I; PTPS: 6-piruvoil tetrahidrpterina sintasa; PTPR: 6-piruvoil tetrahidrpterina 2'-reductasa; SR: sepiapterina reductasa; DHFR: dihidrofolato reductasa. 


\subsection{Vitiligo}

El vitiligo es una patología cutánea muy frecuente en los seres humanos. Consiste en un desorden en la pigmentación que produce manchas blancas sobre la piel. Estas manchas muestran una característica fluorescencia cuando son sometidas al denominado examen con luz de Wood (351 nm). Se ha demostrado que este fenómeno ocurre por la acumulación de pterinas oxidadas en las zonas afectadas por la enfermedad, 39,44 ya que son compuestos muy fluorescentes. 45 Por este motivo resulta interesante comentar el papel que juegan varios derivados pterínicos en esta patología.

Los pacientes con vitiligo presentan un gran aumento en la síntesis de novo $\mathrm{H}_{4} \mathrm{Bip}$, y una alteración de la ruta de reciclado de este cofactor. ${ }^{46-48} \mathrm{El}$ aumento de los niveles de este tetrahidroderivado lleva a la acumulación de $\mathrm{H}_{2} \mathrm{Bip}$ y 7- $\mathrm{H}_{4} \mathrm{Bip}$ (un isómero de $\left.\mathrm{H}_{4} \mathrm{Bip}\right){ }^{49}$ Este último es un potente inhibidor de la actividad de la primer enzima en la ruta de síntesis de melanina (PAH). ${ }^{50}$ Como consecuencia de la baja actividad de $\mathrm{PAH}$, aumentan los niveles de fenilalanina en piel, favoreciendo la síntesis de $\mathrm{H}_{4} \mathrm{Bip}$ y aumentando aún más los niveles del tetrahidroderivado. El incremento de los niveles de fenilalanina y de 7- $\mathrm{H}_{4} \mathrm{Bip}$, junto a la disminución de la actividad enzimática lleva a la generación y acumulación de $\mathrm{H}_{2} \mathrm{O}_{2}$ en la piel de estos pacientes (Esquema 2.6). ${ }^{50,51}$ Esta acumulación conduce a la inactivación de la enzima catalasa, ${ }^{52}$ y motivo por el cual en los melanocitos de los pacientes con vitiligo el medio se vuelve muy oxidante, alcanzando concentraciones de $\mathrm{H}_{2} \mathrm{O}_{2}$ del orden de milimolar. El incremento de los niveles de especies oxidantes en las manchas depigmentadas quedó demostrado por biopsias de piel, en las que se encuentra un alto grado de vacuolización y peroxidación lipídica.

Si bien ciertos autores postulan la oxidación de $\mathrm{H}_{4} \mathrm{Bip}$ y $\mathrm{H}_{2} \mathrm{Bip}$ a 6-biopterina (6Bip) y de 7- $\mathrm{H}_{4} \mathrm{Bip}$ a 7-biopterina (7-Bip) por parte de $\mathrm{H}_{2} \mathrm{O}_{2}, 53,54$ Dantola et al. demostraron que, $\mathrm{H}_{2}$ Bip es oxidada por esta especie reactiva de oxígeno dando como principal producto $\mathrm{H}_{2} \mathrm{Xap} .{ }^{25}$ Esto último conduce al interrogante de cuál es el origen de la presencia de pterinas oxidadas en la piel de los pacientes que sufren esta enfermedad. Una vía importante que merece ser investigada es la degradación fotoquímica de $\mathrm{H}_{2} \mathrm{Bip}$. Si bien no es claro cuál es el primer mecanismo que perturba la homeostasis de $\mathrm{H}_{4} \mathrm{Bip}$, se sabe que estas alteraciones metabólicas conducen a una situación en la cual se tiene un tejido expuesto a la luz solar, sin protección contra la misma y donde se acumulan 
metabolitos fotoquímicamente activos. En el próximo capítulo se describirán algunas de las reacciones que pueden estar ocurriendo en el interior de los melanocitos.

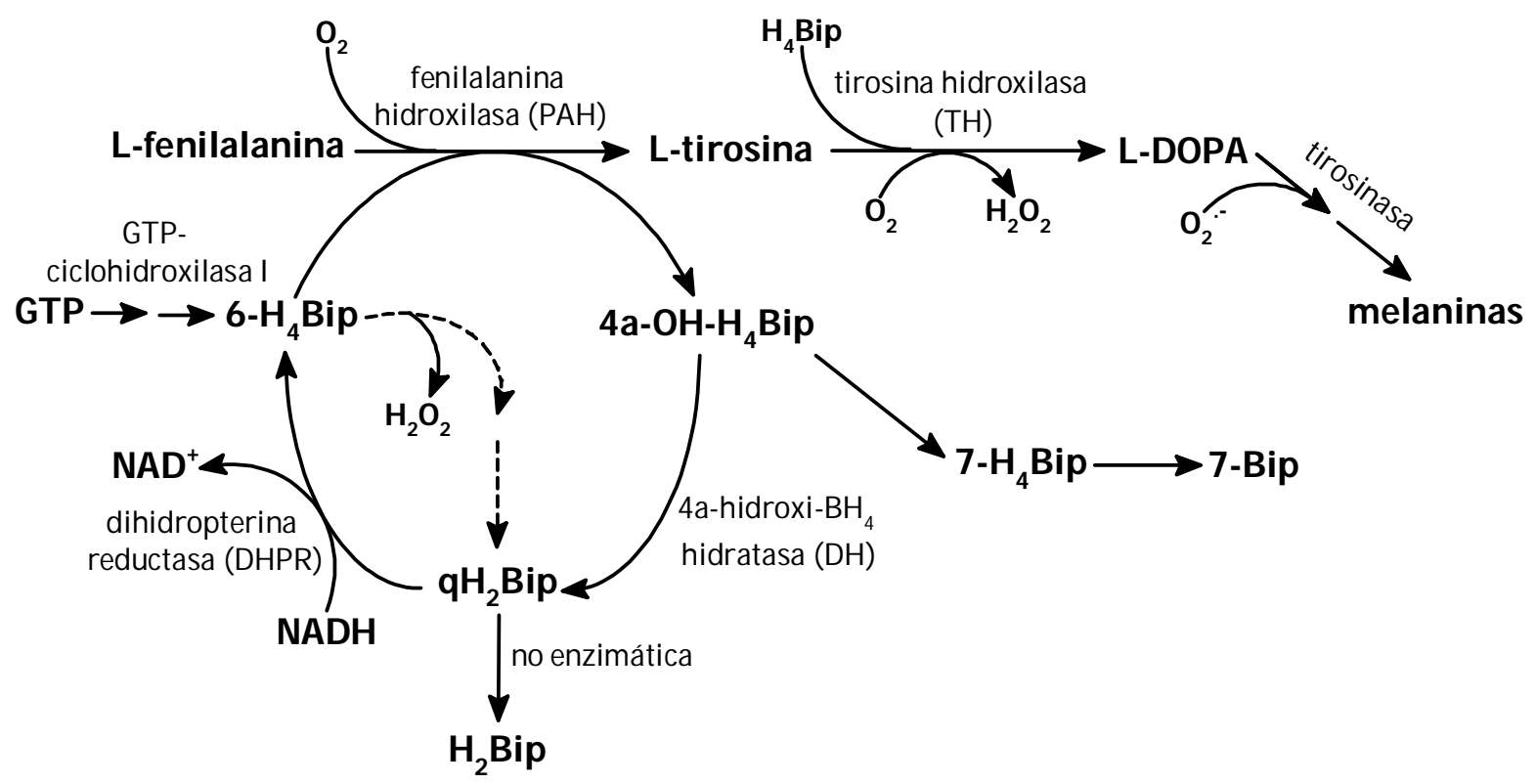

Esquema 2.6 Síntesis de novo, reciclado y regulación de la síntesis de $\mathrm{H}_{4} B i p$ que tiene lugar en la melanogénesis. Las flechas punteadas indican la ruta corta de reciclado de $\mathrm{H}_{4} \mathrm{Bip}$, favorecida en el vitiligo.

\subsection{Neopterina y dihidroneopterina}

Nep y su forma reducida $\mathrm{H}_{2} \mathrm{Nep}$ son ambos intermediarios de la biosíntesis de $\mathrm{H}_{4} \mathrm{Bip}$, sintetizada a partir de guanosina trifosfato (GTP) en una reacción catalizada por la GTP-ciclohidrolasa I (Esquema 2.5). No obstante, su función más importante es la participación en procesos inmunológicos. Los linfocitos y los macrófagos son dos tipos de células del sistema inmune que juegan un papel fundamental cuando se activa la respuesta inmune celular en las distintas enfermedades. Los linfocitos- $T$ reconocen antígenos en la superficie de las células de los tejidos, se activan y producen citoquinas, particularmente interferón $\gamma$ (INF- $\gamma$ ). El INF- $\gamma$ estimula a los macrófagos quienes, a su vez, sintetizan y liberan al medio, entre otras sustancias, especies reactivas de oxígeno (EROs), factor de necrosis tumoral (TNF- $\alpha$ ), $\mathrm{H}_{2}$ Nep y Nep. 55

$\mathrm{Al}$ parecer, los macrófagos sintetizan $\mathrm{H}_{2} \mathrm{Nep}$ como un antioxidante celular. Esto se debe a que, durante el proceso inflamatorio, los macrófagos requieren antioxidantes adicionales para protegerse y poder funcionar en los sitios de inflamación donde hay altas concentraciones de especies reactivas como $\mathrm{H}_{2} \mathrm{O}_{2}$, $\mathrm{ClO}^{-}$y $\mathrm{O}_{2}{ }^{-} .56$ Asimismo, $\mathrm{H}_{2} \mathrm{Nep}$ y Nep participan en otras funciones de interés biológico. Nep favorece la liberación del factor de transcripción NF- $\kappa B^{57}$ el cual participa estimulando a las células para que 
aumenten la liberación de NO• y promueve la transcripción del promotor del virus HIV-1 y, por lo tanto, la expresión del mismo. ${ }^{58}$

Al ser Nep liberada en la respuesta inmune, su concentración en sangre se ve aumentada en infecciones virales (hepatitis A, B y C, HIV y sarampión), así como también, en infecciones por bacterias intracelulares (tuberculosis, lepra, etc.) y parásitos intracelulares (malaria). De esta manera, la determinación de la concentración de Nep en distintos fluidos biológicos (suero, fluido cerebroespinal u orina) es una herramienta muy útil para el monitoreo de la respuesta inmunológica mediada por células. ${ }^{59}$ Estos resultados indican que Nep es un marcador inespecífico de respuesta inmunológica, pero es importante destacar que el aumento en los niveles de Nep, generalmente precede a los síntomas clínicos y correlacionan bien con la actividad de la enfermedad. Consecuentemente, su determinación se utiliza como prueba de screening en bancos de sangre, marcador pronóstico de enfermedades cardiovasculares, 60 sepsis, síndrome de respuesta inflamatoria sistémica, ${ }^{61}$ neoplasias. ${ }^{62}$ Además, Nep es un indicador sensible de las complicaciones inmunológicas tempranas, como rechazos o infecciones en receptores de trasplantes. También puede usarse como indicador de estrés oxidativo, ya que la concentración de Nep se correlaciona con productos de oxidación de proteínas y con la pérdida de antioxidantes (vitamina E).

\subsection{Referencias}

1. F. G. Hopkins, Nature, 1889, 40, 335.

2. F. G. Hopkins, Proceedings of the Chemical Society, London, 1889, 5, 117.

3. F. G. Hopkins, The Pigments of the Pieridae: A Contribution to the Study of Excretory Substances Which Function in Ornament, Philosophical Transactions of the Royal Society of London. B, 1895, 186, 661-682.

4. R. Purrmann, Über die Flügelpigmente der Schmetterlinge. VII. Synthese des Leukopterins und Natur des Guanopterins, Justus Liebigs Annalen der Chemie, 1940, 544, 182-190.

5. R. Purrmann, Konstitution und Synthese des sogenannten Anhydroleukopterins. Über die Flügelpigmente der Schmetterlinge XII, Justus Liebigs Annalen der Chemie, 1941, 548, 284-292.

6. C. Schöpf and R. Reichert, Zur Kenntnis des Leukopterins, Justus Liebigs Annalen der Chemie, 1941, 548, 82-94.

7. W. Pfleiderer, Chemistry and properties of dihydropterins, in Biochemical and Clinical Aspects of Pteridines, ed. W. Pfleiderer, H. Wachter and A. J. Blair, Walter de Gruyter \& Co., Berlin, New York, 1987, pp. 3-21.

8. W. Pfleiderer, Chemistry of dihydropterins and tetrahydropterins, Journal of Inherited Metabolic Disease, 1978, 1, 54-60.

9. A. Albert, Quantitative studies of the avidity of naturally occurring substances for trace metals. III. Pteridines, riboflavin and purines, Biochem. J., 1953, 54, 646-640.

10. V. D. Monópoli, A. H. Thomas and A. L. Capparelli, Kinetics and equilibrium study of nickel(II) complexation by pterin, International Journal of Chemical Kinetics, 2000, 32, 231-237.

11. A. H. Thomas, G. Suárez, F. M. Cabrerizo, R. Martino and A. L. Capparelli, Study of the photolysis of folic acid and 6-formylpterin in acid aqueous solutions, Journal of Photochemistry and Photobiology A: Chemistry, 2000, 135, 147-154. 
12. A. H. Thomas, M. R. Feliz and A. L. Capparelli, Equilibrium and kinetic studies of cobalt(II) complexation by folic acid, Transition Metal Chemistry, 1996, 21, 317-321.

13. C. Lorente, A. L. Capparelli, A. H. Thomas, A. M. Braun and E. Oliveros, Quenching of the fluorescence of pterin derivatives by anions, Photochemical and Photobiological Sciences, 2004, 3, 167-173.

14. G. Maharaj, B. S. Selinsky, J. R. Appleman, M. Perlman, R. E. London and R. L. Blakley, Dissociation constants for dihydrofolic acid and dihydrobiopterin and implications for mechanistic models for dihydrofolate reductase, Biochemistry, 1990, 29, 4554-4560.

15. M. L. Dántola, Reactividad térmica y fotoquímica de 7,8-dihidropterinas frente a especies oxidantes en condiciones fisiológicas, in Departamento de Química - Facultad de Ciencias Exactas, Universidad Nacional de La Plata, La Plata, 2008, p. 250.---

16. M. L. Dántola, M. Vignoni, A. L. Capparelli, C. Lorente and A. H. Thomas, Stability of 7,8-dihydropterins in air-equilibrated aqueous solutions, Helvetica Chimica Acta, 2008, 91, 411-425.

17. F. M. Cabrerizo, C. Lorente, M. Vignoni, R. Cabrerizo, A. H. Thomas and A. L. Capparelli, Photochemical behavior of 6-methylpterin in aqueous solutions: Generation of reactive oxygen species, Photochemistry and Photobiology, 2005, 81, 793-801.

18. F. M. Cabrerizo, A. H. Thomas, C. Lorente, M. L. Dántola, G. Petroselli, R. Erra-Balsells and A. L. Capparelli, Generation of Reactive Oxygen Species during the Photolysis of 6-(Hydroxymethyl)pterin in Alkaline Aqueous Solutions, Helvetica Chimica Acta, 2004, 87, 349-365.

19. C. Lorente, Fotofísica y propiedades fotosensibilizadoras de pterinas en solución acuosa, in Departamento de Química - Facultad de Ciencias Exactas, Universidad Nacional de La Plata, La Plata, 2003, p. 198.---

20. F. M. Cabrerizo, Propiedades fotofísicas y fotoquímicas de pterinas en solución acuosa: fluorescencia, producción de especies reactivas de oxígeno y oxidación, in Departamento de Química - Facultad de Ciencias Exactas, Universidad Nacional de La Plata, La Plata, 2005, p. 335.---

21. R. Baur, M. Kappel, R. Mengel and W. Pfleiderer, Photochemistry of pteridines, in Chemistry and Biology of Pteridines, ed. R. L. Kisliuk and G. M. Brown, Elsevier, New York, 1979, pp. 13-18.

22. W. Pfleiderer, M. Kappel and R. Baur, Biochemical and Clinical Aspects of Pteridines, Walter de Gruyter \& Co., Berlin - New York, 1984.

23. A. H. Thomas, G. Suárez, F. M. Cabrerizo and A. L. Capparelli, Photochemistry of 6-formylpterin in alkaline medium, Helvetica Chimica Acta, 2001, 84, 3849-3860.

24. A. H. Thomas, G. Suárez, F. M. Cabrerizo, F. S. García Einschlag, R. Martino, C. Baiocchi, E. Pramauro and A. L. Capparelli, Photochemical behavior of folic acid in alkaline aqueous solutions and evolution of its photoproducts, Helvetica Chimica Acta, 2002, 85, 2300-2315.

25. M. L. Dántola, T. M. Schuler, M. P. Denofrio, M. Vignoni, A. L. Capparelli, C. Lorente and A. H. Thomas, Reaction between 7,8-dihydropterins and hydrogen peroxide under physiological conditions, Tetrahedron, 2008, 64, 8692-8699.

26. T. Ohue, K. Koshimura, K. Lee, Y. Watanabe and S. Miwa, A novel action of 6R-l-erythro-5,6,7,8tetrahydrobiopterin, a cofactor for hydroxylases of phenylalanine, tyrosine and tryptophan: Enhancement of acetylcholine release in vivo in the rat hippocampus, Neuroscience Letters, 1991, 128, 93-96.

27. R. E. Frye, L. C. Huffman and G. R. Elliott, Tetrahydrobiopterin as a Novel Therapeutic Intervention for Autism, Neurotherapeutics, 2010, 7, 241-249.

28. K. Schmidt, E. R. Werner, B. Mayer, H. Wachter and W. R. Kukovetz, Tetrahydrobiopterin-dependent formation of endothelium-derived relaxing factor (nitric oxide) in aortic endothelial cells, Biochem. J., 1992, 281, 297-300.

29. S. S. Gross and R. Levi, Tetrahydrobiopterin synthesis. An absolute requirement for cytokine-induced nitric oxide generation by vascular smooth muscle, Journal of Biological Chemistry, 1992, 267, 2572225729.

30. C. Sumi-Ichinose, F. Urano, R. Kuroda, T. Ohye, M. Kojima, M. Tazawa, H. Shiraishi, Y. Hagino, T. Nagatsu, T. Nomura and H. Ichinose, Catecholamines and Serotonin Are Differently Regulated by Tetrahydrobiopterin, Journal of Biological Chemistry, 2001, 276, 41150-41160.

31. A. Reif, L. G. Fröhlich, P. Kotsonis, A. Frey, H. M. Bömmel, D. A. Wink, W. Pfleiderer and H. H. H. W. Schmidt, Tetrahydrobiopterin Inhibits Monomerization and Is Consumed during Catalysis in Neuronal NO Synthase, Journal of Biological Chemistry, 1999, 274, 24921-24929.

32. P. Klatt, M. Schmid, E. Leopold, K. Schmidt, E. R. Werner and B. Mayer, The pteridine binding site of brain nitric oxide synthase. Tetrahydrobiopterin binding kinetics, specificity, and allosteric interaction with the substrate domain, Journal of Biological Chemistry, 1994, 269, 13861-13866.

33. C. R. Nishida and P. R. O. de Montellano, Electron Transfer and Catalytic Activity of Nitric Oxide Synthases, Journal of Biological Chemistry, 1998, 273, 5566-5571. 
34. M. A. Marletta, Nitric oxide synthase: Function and mechanism, Advances in Experimental Medicine and Biology, 1993, 338, 281-284.

35. M. J. Crabtree, C. L. Smith, G. Lam, M. S. Goligorsky and S. S. Gross, Ratio of 5,6,7,8-tetrahydrobiopterin to 7,8-dihydrobiopterin in endothelial cells determines glucose-elicited changes in NO vs. superoxide production by eNOS, American Journal of Physiology - Heart and Circulatory Physiology, 2008, 294, H1530-H1540.

36. N. S. Kwon, C. F. Nathan and D. J. Stuehr, Reduced biopterin as a cofactor in the generation of nitrogen oxides by murine macrophages, Journal of Biological Chemistry, 1989, 264, 20496-20501.

37. K. U. Schallreuter, J. M. Wood, C. Körner, K. M. Harle, V. Schulz-Douglas and E. R. Werner, 6Tetrahydrobiopterin functions as a UVB-light switch for de novo melanogenesis, Biochimica et Biophysica Acta (BBA) - Protein Structure and Molecular Enzymology, 1998, 1382, 339-344.

38. K. Jimbow, M. A. Pathak and T. B. Fitzpatrick, Effect of ultraviolet on the distribution pattern of microfilaments and microtubules and on the nucleus in human melanocytes, Yale Journal of Biology and Medicine 1973, 46, 411-426.

39. K. U. Schallreuter, J. M. Wood, M. R. Pittelkow, M. Gütlich, K. R. Lemke, W. Rödl, N. N. Swanson, K. Hitzemann and I. Ziegler, Regulation of melanin biosynthesis in the human epidermis by tetrahydrobiopterin Science, 1994, 263, 1444-1446.

40. K. U. Schallreuter, V. Schulz-Douglas, A. Bünz, W. D. Beazley and C. Körner, Pteridines in the Control of Pigmentation, Journal of Investiga tive Dermatology, 1997, 109, 31-35.

41. C. A. Nichol, G. K. Smith and D. S. Duch, Biosynthesis and metabolism of tetrahydrobiopterin and molybdopterin, Annu. Rev. Biochem., 1985, 54, 729-764.

42. K. Sawabe, Y. Suetake, K. O. Wakasugi and H. Hasegawa, Accumulated BH4 in mouse liver caused by administration of either 6R- or 6SBH4 consisted solely of the 6R-diastereomer: Evidence of oxidation to BH2 and enzymic reduction, Molecular Genetics and Metabolism, 2005, 86, Supplement, 145-147.

43. H. Hasegawa, K. Sawabe, N. Nakanishi and O. K. Wakasugi, Delivery of exogenous tetrahydrobiopterin (BH4) to cells of target organs: Role of salvage pathway and uptake of its precursor in effective elevation of tissue BH4, Molecular Genetics and Metabolism, 2005, 86, Supplement, 2-10.

44. K. U. Schallreuter, J. M. Wood, I. Ziegler, K. R. Lemke, M. R. Pittelkow, N. J. Lindsey and M. Gütlich, Defective tetrahydrobiopterin and catecholamine biosynthesis in the depigmentation disorder vitiligo, Biochimica et Biophysica Acta, 1994, 1226, 181-192.

45. C. Lorente and A. H. Thomas, Photophysics and photochemistry of pterins in aqueous solution, Accounts of Chemical Research, 2006, 39, 395-402.

46. S. Kaufman, A Protein That Stimulates Rat Liver Phenylalanine Hydroxylase, Journal of Biological Chemistry, 1970, 245, 4751-4759.

47. C. Y. Huang, E. E. Max and S. Kaufman, Purification and Characterization of Phenylalanine Hydroxylase-stimulating Protein from Rat Liver, Journal of Biological Chemistry, 1973, 248, 42354241.

48. J. E. Ayling, I. Rebrin, B. Thöny and S. W. Bailey, Mechanism of Dehydration by the Bifunctional Protein, 4a-Hydroxy-tetrahydropterin Dehydratase/ $\mathrm{DCoH}$, in Chemistry and Biology of Pteridines and Folates, ed. W. Pfleiderer and R. Rokos, Blackwell Science, Berlin, 1997, pp. 565-570.

49. H. C. Curtius, A. Matasovic, G. Schoedon, T. Kuster, P. Guibaud, T. Giudici and N. Blau, 7-Substituted pterins. A new class of mammalian pteridines, Journal of Biological Chemistry, 1990, 265, 3923-3930.

50. M. D. Davis, P. Ribeiro, J. Tipper and S. Kaufman, "7-tetrahydrobiopterin," a naturally occurring analogue of tetrahydrobiopterin, is a cofactor for and a potential inhibitor of the aromatic amino acid hydroxylases, Proceedings of the National Academy of Sciences, 1992, 89, 10109-10113.

51. K. U. Schallreuter, J. Moore, J. M. Wood, W. D. Beazley, D. C. Gaze, D. J. Tobin, H. S. Marshall, A. Panske, E. Panzig and N. A. Hibberts, In vivo and in vitro evidence for hydrogen peroxide $\left(\mathrm{H}_{2} \mathrm{O}_{2}\right)$ accumulation in the epidermis of patients with vitiligo and its successful removal by a UVB-activated pseudocatalase, Journal of Investigative Dermatology Symposium Proceedings, 1999, 4, 91-96.

52. S. Aronoff, Catalase: Kinetics of Photooxidation, Science, 1965, 150, 72-73.

53. H. Rokos, W. D. Beazley and K. U. Schallreuter, Oxidative stress in vitiligo: Photo-oxidation of pterins produces $\mathrm{H}_{2} \mathrm{O}_{2}$ and pterin-6-carboxylic acid, Biochem. Biophys. Res. Commun., 2002, 292, 805-811.

54. J. Moore, J. M. Wood and K. U. Schallreuter, $\mathrm{H}_{2} \mathrm{O}_{2}$-mediated oxidation of tetrahydrobiopterin: Fourier transform Raman investigations provide mechanistic implications for the enzymatic utilization and recycling of this essential cofactor, Journal of Raman Spectroscopy, 2002, 33, 610-617.

55. C. Huber, J. R. Batchelor and D. Fuchs, Immune response-associated production of neopterin. Release from macrophages primarily under control of interferon-gamma, J. Exp. Med., 1984, 160, 310-316.

56. S. P. Gieseg, G. Reibnegger, H. Wachter and H. Esterbauer, 7,8 Dihydroneopterin inhibits low density lipoprotein oxidation in vitro. Evidence that this macrophage secreted pteridine is an anti-oxidant, Free Radical Research, 1995, 23, 123-136. 
57. G. Hoffmann, W. Schobersberger, S. Frede, L. Pelzer, J. Fandrey, H. Wachter, D. Fuchs and J. r. Grote, Neopterin activates transcription factor nuclear factor- $\mathrm{\kappa B}$ in vascular smooth muscle cells, FEBS Letters, 1996, 391, 181-184.

58. G. Baier-bitterlich, D. Fuchs and H. Wachter, Chronic immune stimulation, oxidative stress, and apoptosis in HIV infection, Biochemical Pharmacology, 1997, 53, 755-763.

59. D. Fuchs, G. Weiss, G. Reibnegger and H. Wachter, The Role of Neopterin as a Monitor of Cellular Immune Activation in Transplantation, Inflammatory, Infectious, and Malignant Diseases, Critical Reviews in Clinical Laboratory Sciences, 1992, 29, 307-344.

60. S. de Rosa, P. Cirillo, M. Pacileo, G. Petrillo, G. L. D'Ascoli, F. Maresca, F. Ziviello and M. Chiariello, Neopterin: From Forgotten Biomarker to Leading Actor in Cardiovascular Pathophysiology, Current Vascular Pharmacology, 2011, 9, 188-199.

61. G. Girgin, T. T. Sahin, D. Fuchs, O. Yuksel, O. Kurukahvecioglu, M. Sare and T. Baydar, Tryptophan degradation and serum neopterin concentrations in intensive care unit patients, Toxicology Mechanisms and Methods, 2011, 21, 231-235.

62. C. Murr, G. Neurauter, B. Wirleitner and D. Fuchs, Neopterin to predict prognosis of infection and neoplasia, in International EUROGIN-EAST conference Vilnius, 2001.--- 


\section{Capítulo 3}

\section{Fotoquímica de pterinas}

Desde hace varias décadas se sabe que ciertas pterinas oxidadas y también algunos derivados reducidos son fotosensibles cuando los mismos se encuentran disueltos en solución acuosa. Por lo tanto, en este capítulo se resumen los conocimientos existentes, hasta el momento previo al inicio de este trabajo de tesis, sobre el comportamiento fotofísico y fotoquímico de distintos derivados pterínicos.

\subsection{Estados excitados singletes y tripletes de pterinas}

Existen diversos estudios sobre los estados excitados de los distintos compuestos pterínicos, ya sea pterinas oxidadas o los dihidroderivados. Las características fluorescentes de las pterinas aromáticas (espectros de emisión y excitación, rendimiento cuántico de emisión $\left(\Phi_{\mathrm{F}}\right)$ y tiempo de vida $\left(\tau_{\mathrm{F}}\right)$ ) dependen de varios factores. ${ }^{1-3}$ Por un lado, varían según sea la naturaleza química del sustituyente unido en la posición 6 del anillo pterínico. Mientras las denominadas pterinas conjugadas (Capítulo 2) poseen una fluorescencia despreciable, las no conjugadas presentan, por el contrario, una emisión importante. Por otra parte, la emisión depende del pH, es decir, de cuál sea la forma ácido-base del derivado pterínico presente en el medio de reacción. Típicamente, los espectros de emisión de las formas alcalinas presentan un corrimiento de 10 - $15 \mathrm{~nm}$ hacia el visible respecto de los espectros de emisión de las correspondientes formas ácidas, lo cual es esperable considerando los espectros de absorción (Sección 2.2). Además, estos espectros normalizados de las pterinas no conjugadas son independientes de la longitud de onda de excitación, sugiriendo que la emisión fluorescente solo tiene lugar desde el estado excitado singlete de menor energía (S1).

En la Tabla 3.1 se exponen los valores publicados de $\Phi_{\mathrm{F}} \mathrm{y} \tau_{\mathrm{F}}$ de varias pterinas aromáticas. Puede apreciarse que, en todos los casos, los valores de $\Phi_{\mathrm{F}}$ correspondientes a las formas ácidas son mayores que los valores de las formas alcalinas. Además, estos valores presentan una fuerte dependencia con la naturaleza del sustituyente. Ciertamente, la presencia de la porción p-aminobenzoico provoca en las pterinas conjugadas una drástica reducción de los valores de $\Phi_{\mathrm{F}}$. Asimismo, los decaimientos de 
fluorescencia de las pterinas siguen un comportamiento exponencial de primer de orden y puede observarse que los $\tau_{\mathrm{F}}$ para las formas ácidas son mayores que los de sus respectivas formas alcalinas y que también tiene una clara dependencia con el tipo de sustituyente en la posición 6. En la Tabla 3.2 puede observarse que los valores de $\Phi_{\mathrm{F}} \mathrm{y} \tau_{\mathrm{F}}$ obtenidos para distintos dihidroderivados son, ciertamente, muchos menores que los observados para los análogos oxidados. Este hecho da cuenta de la existencia para los dihidroderivados, de vías adicionales de desactivación no radiativa de los estados excitados singletes muy eficientes.

\begin{tabular}{|c|c|c|c|c|c|}
\hline Compuesto & forma & $\lambda_{\mathrm{F}} / \mathrm{nm}( \pm 5)$ & $\Phi_{\mathrm{F}}$ & $\tau_{\mathrm{F}} / \mathrm{ns}( \pm 0,4)$ & $\Phi_{\Delta}$ \\
\hline \multirow{2}{*}{ Bip } & Ácida & 441 & $0,36 \pm 0,01$ & 9,1 & $0,34 \pm 0,01$ \\
\hline & Básica & 455 & $0,29 \pm 0,01$ & 7,6 & $0,40 \pm 0,03$ \\
\hline \multirow{2}{*}{ Nep } & Ácida & 440 & $0,38 \pm 0,01$ & 8,9 & $0,23 \pm 0,01$ \\
\hline & Básica & 454 & $0,31 \pm 0,01$ & 7,4 & $0,34 \pm 0,04$ \\
\hline \multirow{2}{*}{ Ptr } & Ácida & 439 & $0,33 \pm 0,01$ & 7,6 & $0,18 \pm 0,02$ \\
\hline & Básica & 456 & $0,27 \pm 0,01$ & 5,0 & $0,30 \pm 0,02$ \\
\hline \multirow{2}{*}{ Fop } & Ácida & 446 & $0,12 \pm 0,01$ & 7,9 & $0,45 \pm 0,05$ \\
\hline & Básica & 454 & $0,07 \pm 0,01$ & 2,2 & $0,47 \pm 0,02$ \\
\hline \multirow{2}{*}{ Cap } & Ácida & 439 & $0,28 \pm 0,01$ & 5,8 & $0,27 \pm 0,03$ \\
\hline & Básica & 451 & $0,18 \pm 0,01$ & 4,1 & $0,37 \pm 0,02$ \\
\hline \multirow{2}{*}{ Mep } & Ácida & 448 & $0,61 \pm 0,01$ & 13,6 & $0,10 \pm 0,02$ \\
\hline & Básica & 460 & $0,61 \pm 0,04$ & 13,2 & $0,14 \pm 0,02$ \\
\hline \multirow{2}{*}{ Hmp } & Ácida & 449 & $0,53 \pm 0,02$ & 11,0 & $0,15 \pm 0,02$ \\
\hline & Básica & 457 & $0,46 \pm 0,01$ & 8,4 & $0,21 \pm 0,01$ \\
\hline \multirow{2}{*}{ PteGlu } & Ácida & 445 & $<0,005$ & - & $\varangle 0,02$ \\
\hline & Básica & 455 & $<0,005$ & - & $\varangle 0,02$ \\
\hline
\end{tabular}

Tabla 3.1 Longitud de onda del máximo de emisión $\left(\lambda_{\mathrm{F}}\right)$, rendimiento cuántico de fluorescencia $\left(\Phi_{\mathrm{F}}\right)$ tiempo de vida de fluorescencia $\left(\tau_{\mathrm{F}}\right)$, y rendimientos cuánticos de producción de ${ }^{1} \mathrm{O}_{2}\left(\Phi_{\Delta}\right)$ de las formas ácidas y alcalinas de algunos derivados pterínicos aromáticos. Datos extraídos de Ref. 1-3.

\begin{tabular}{cccc}
\hline Compuesto & $\lambda_{\mathrm{F}} / \mathbf{n m}(\mathbf{\pm})$ & $\Phi_{\mathrm{F}}$ & $\tau_{\mathrm{F}} / \mathbf{n s} \mathbf{( \pm \mathbf { 0 , 0 7 } )}$ \\
\hline $\mathrm{H}_{2}$ Bip & 425 & $0,009 \pm 0,002$ & 0,30 \\
$\mathrm{H}_{2} \mathrm{Nep}$ & 425 & $0,005 \pm 0,001$ & 0,31 \\
$\mathrm{H}_{2} \mathrm{Hmp}$ & 425 & $0,003 \pm 0,001$ & 0,21 \\
$\mathrm{H}_{2}$ Mep & 410 & $0,003 \pm 0,001$ & 0,18 \\
$\mathrm{H}_{2} \mathrm{Fop}$ & 528 & $0,0087 \pm 0,0003$ & 0,34 \\
Sep & 533 & $0,0070 \pm 0,0006$ & 0,28
\end{tabular}

Tabla 3.2 Rendimiento cuántico $\left(\Phi_{\mathrm{F}}\right)$ y tiempo de vida $\left(\tau_{\mathrm{F}}\right)$ de fluorescencia de dihidroderivados. Datos extraídos de Ref. 4. 
También se conoce que las pterinas aromáticas son eficientes fotosensibilizadores de ${ }^{1} \mathrm{O}_{2} \cdot{ }^{5-7}$ En solución acuosa y bajo irradiación UV-A, todas las pterinas aromáticas poseen una elevada eficiencia de producción de ${ }^{1} \mathrm{O}_{2}$. Por el contrario, las pterinas conjugadas como el ácido fólico (PteGlu), no generan esta especie reactiva. Los valores de rendimientos cuánticos de producción de ${ }^{1} \mathrm{O}_{2}\left(\Phi_{\Delta}\right)$ se detallan en la Tabla 3.1. Para explicar el comportamiento diferencial observado con PteGlu se propuso que la cadena lateral de este compuesto actúa como desactivador interno, aumentando la velocidad de desexcitación no radiativa (conversión interna) del estado excitado singlete. De esta manera, se observa emisión muy débil y el entrecruzamiento de sistemas es ineficiente, por lo cual, no genera ${ }^{1} \mathrm{O}_{2}$. Se aprecia, además, que tanto la naturaleza del sustituyente en la posición 6 del anillo pterínico como el pH, afectan significativamente los valores de $\Phi_{\Delta}$, siendo mayor en medio alcalino para la mayoría de las pterinas estudiadas.

Asimismo, existen algunos estudios sobre los transientes de absorción de pterinas por técnicas de laser flash photolysis. En estos trabajos se postula que al excitar una solución de pterina (Ptr) a 355 nm se observan, en simultáneo, especies tripletes y radicales. Chahidi et al. ${ }^{8}$ asignaron dos de estos transientes a estados tripletes de Ptr. Uno de ellos tiene un máximo de absorción a $550 \mathrm{~nm}$ y un tiempo de vida $\left(\tau_{\mathrm{T}}\right)$ de 2,3 \pm 0,2 $\mu \mathrm{s}$. El otro transiente posee dos máximos de absorción a 415 y $600 \mathrm{~nm}$ y un $\tau_{\mathrm{T}}$ de 0,3 $\mu$ s. La existencia de dos estados tripletes se debe, según estos autores, al equilibrio tautomérico presente en el estado fundamental de Ptr (Capítulo 2). Ledbetter et al. ${ }^{9}$ propusieron una hipótesis similar para los resultados obtenidos con biopterina (Bip), encontrando dos estados excitados tripletes con $\tau_{\mathrm{T}}$ de $2,0 \mu$ s y $0,3 \mu \mathrm{s}$.

Por otra parte, los dihidroderivados, a diferencia de las pterinas aromáticas, poseen capacidad extremadamente baja o nula para generar fotoquímicamente ${ }^{1} \mathrm{O}_{2} \cdot{ }^{10}$ Teniendo en cuenta además que poseen $\tau_{\mathrm{T}}$ muy cortos, se concluyó que los estados excitados singletes de los dihidroderivados se desactivan muy rápidamente mediante vías no radiativas, impidiendo de esa forma la generación de estados excitados tripletes.

Es interesante comentar que las dihidropterinas que se encuentran presentes en los organismos en condiciones fisiológicas, incluso en zonas expuestas a la radiación como la piel del ser humano, cuando sus estados excitados pierden el exceso de energía lo hacen muy rápidamente, evitando así la formación de especies reactivas capaces de 
reaccionar con otros compuestos presentes en el medio. Por el contrario, las pterinas aromáticas no conjugadas que solo aparecen en piel bajo condiciones patológicas (Sección 2.5), generan fotoquímicamente especies como los estados excitados tripletes que viven lo suficiente como para reaccionar y, además, formar la especie altamente reactiva ${ }^{1} \mathrm{O}_{2}$.

\subsection{Reactividad fotoquímica de las pterinas aromáticas}

En general, en soluciones aireadas y bajo irradiación UV-A, las pterinas aromáticas sufren reacciones de oxidación que involucran modificaciones en el sustituyente localizado en la posición 6, mientras que sus anillos aromáticos permanecen inalterados. Los mecanismos implicados en la fotooxidación de estos compuestos dependen de la naturaleza del sustituyente, como así también del pH del medio. De esta manera, la fotoquímica de la forma ácida y básica de un determinado derivado pterínico puede ser muy diferente.

\subsubsection{Pterinas sin sustituyente oxidable}

Aquellos compuesto pterínicos que no poseen sustituyente oxidable en la posición 6, como Ptr, 6-metilpterina (Mep) o 6-carboxipterina (Cap), son estables cuando se los irradia en solución acuosa en ausencia de $\mathrm{O}_{2}$. En cambio, cuando la irradiación es en presencia de $\mathrm{O}_{2}$ se produce la oxidación de la molécula con la consecuente ruptura de anillo, generando productos no pterínicos y $\mathrm{H}_{2} \mathrm{O}_{2} \cdot{ }^{11}, 12$ No obstante, estas reacciones ocurren muy lentamente, con valores de rendimientos cuánticos mucho menores que los correspondientes a los derivados que sufren oxidación en sus sustituyentes (Tabla 3.3).

\begin{tabular}{cccc}
\hline & \multicolumn{2}{c}{$\Phi$-R $\left(\mathbf{1 0}^{-3}\right)$} & \multirow{2}{*}{ Ref. } \\
\hline medio ácido & medio alcalino & \\
\hline Cap & $0,82 \pm 0,06$ & $1,2 \pm 0,2$ & 11 \\
Mep & $5,1 \pm 0,5$ & $1,3 \pm 0,2$ & 11 \\
Fop & $0,24 \pm 0,05$ & $0,81 \pm 0,08$ & 12 \\
Hmp & $40 \pm 2$ & $35 \pm 2$ & 13,14 \\
PteGlu & $2,3 \pm 0,2$ & $18 \pm 2$ & 6,7 \\
\hline
\end{tabular}

Tabla 3.3 Rendimiento cuántico de consumo $\left(\Phi_{-\mathrm{R}}\right)$ de pterinas aromáticas en soluciones aireadas. 


\subsubsection{Biopterina y neopterina}

En la década del '70, W. Pfleiderer et al. estudiaron la fotoquímica de Bip y neopterina (Nep) en solución buffer de pH 10. En estas condiciones y, bajo irradiación UV-A, los autores observaron que en presencia de $\mathrm{O}_{2}, 6$-formilpterina (Fop) era el producto mayoritario de la reacción. En ausencia de $\mathrm{O}_{2}$, en cambio, detectaron que las soluciones se tornaban de color rojo. Al airear estas soluciones, el compuesto rojo desaparece, detectando Fop como producto final. A este intermediario, con máximo de absorción de su banda de menor energía centrado en $480 \mathrm{~nm}$, se le asignó la estructura de 6-formil-5,8-dihidropterina, un derivado reducido poco estable (Esquema 3.1).16 De esta manera, la reacción involucra la reducción del anillo pterínico y la oxidación del sustituyente a través de una reacción redox intramolecular. En los mencionados trabajos de W. Pfleiderer, si bien se describe que 6-formil-5,8-dihidropterina se oxida rápidamente en presencia de $\mathrm{O}_{2}$, no existe ningún estudio cinético sobre estas reacciones. Más aún, no se encontró en la literatura reportes de constantes de velocidad de reacciones de este tipo.

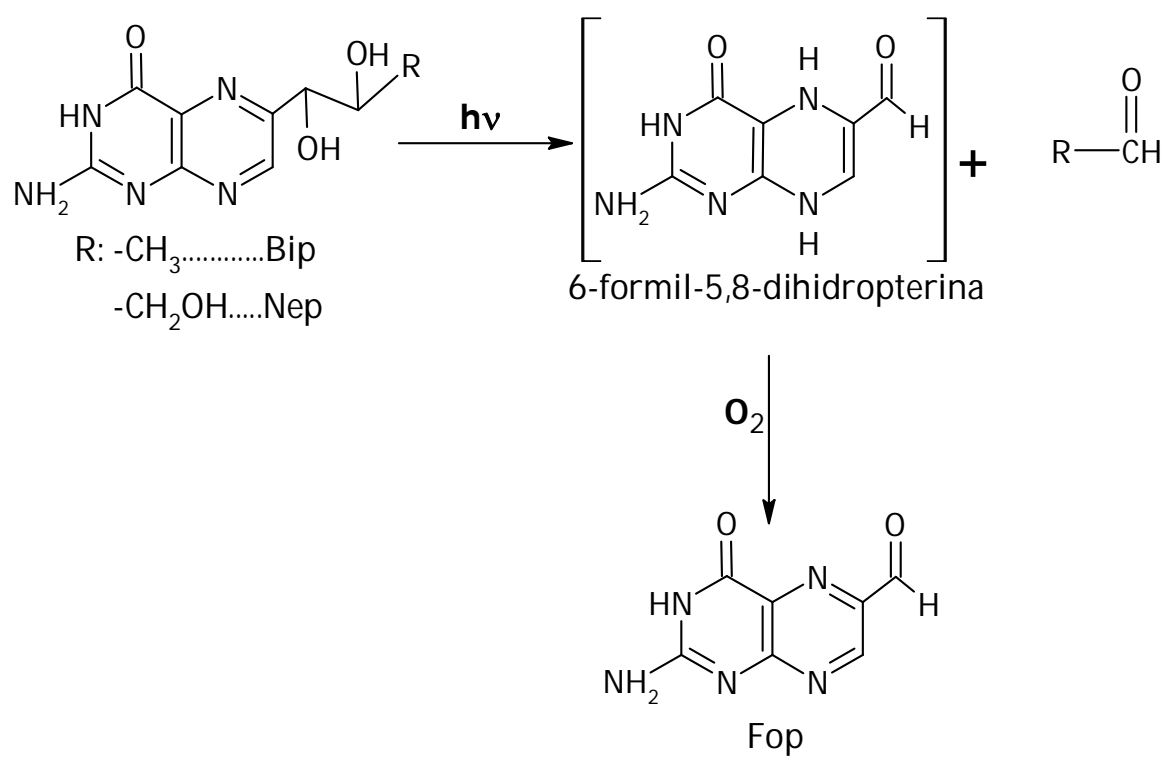

Esquema 3.1 Mecanismo de fotooxidación propuesto por Pfleiderer et al. para Bip y Nep.

\subsubsection{Formilpterina e hidroximetilpterina}

Fop y 6-hidroximetilpterina ( $\mathrm{Hmp}$ ) son otras dos pterinas con sustituyente oxidable cuya reactividad fotoquímica (UV-A) ha sido estudiada. Brevemente, se conoce que Hmp en solución acuosa alcalina genera Fop cuando la misma es irradiada ya sea en presencia o en ausencia de $\mathrm{O}_{2}$. Sin embargo, en condiciones de anaerobiosis se observa 
la formación de un intermediario de características similares a los de Bip y Nep. Este intermediario es oxidado rápidamente por $\mathrm{O}_{2}$, generando Fop y $\mathrm{H}_{2} \mathrm{O}_{2}{ }^{6}$ (Esquema 3.2). Cuando se irradia una solución de Hmp en medio ácido, además de Fop se detecta Cap.7 La fotoquímica de Fop es muy similar a la descripta para Hmp. En presencia de $\mathrm{O}_{2}$, ya sea en medio ácido o alcalino, se oxida el sustituyente del anillo pterínico dando lugar a la formación de Cap y $\mathrm{H}_{2} \mathrm{O}_{2}$. En ausencia de $\mathrm{O}_{2}$ se genera también un intermediario, con características similares a las descriptas para Bip y Nep, que reacciona con $\mathrm{O}_{2}$ para generar como productos finales Cap y $\mathrm{H}_{2} \mathrm{O}_{2} \cdot{ }^{13}, 14$ Los rendimientos cuánticos de consumo de reactivo de Fop y Hmp se detallan en la Tabla 3.3.<smiles>Nc1nc2ncc(CO)nc2c(=O)[nH]1</smiles>
$\mathrm{Hmp}$

b)<smiles>Nc1nc2ncc(C(=O)c3ccccc3)nc2c(=O)[nH]1</smiles>

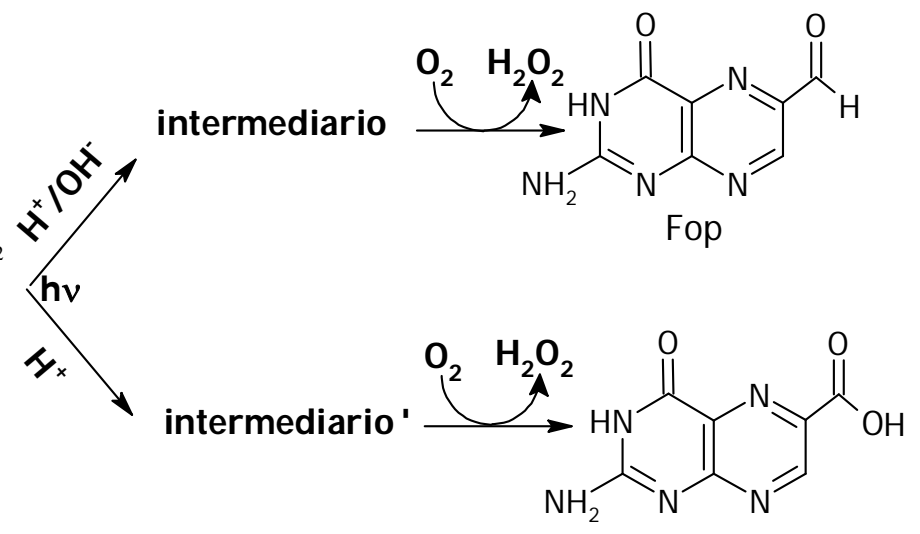

Cap<smiles>CC(=O)C[C@@H](O)[C@H](O)Nc1nc2ncc(C(=O)O)nc2c(=O)[nH]1</smiles>

Esquema 3.2 Mecanismo de fotooxidación propuesto para a) Hmp y b) Fop.

\subsection{4 Ácido fólico}

Cuando PteGlu es irradiado, en medio ácido en presencia de $\mathrm{O}_{2}$, sufre una ruptura generando como fotoproductos Fop y ácido p-aminobenzoilglutámico (PABA-Glu), además de producir $\mathrm{H}_{2} \mathrm{O}_{2} \cdot{ }^{13}$ La velocidad de esta reacción aumenta con el tiempo de irradiación, debido a la existencia de un proceso fotosensibilizado en el cual Fop fotoinduce la oxidación PteGlu. ${ }^{17}$ Posteriormente, Fop se consume generando Cap. En cambio, en la fotooxidación de la forma básica del PteGlu existen dos mecanismos de fotooxidación independientes. El primero es el mismo que sucede en medio ácido. El segundo, es una ruta adicional que no involucra la ruptura del grupo folato y conduce a la formación de un producto que hasta el momento no ha sido identificado (Esquema 
3.3). ${ }^{15}$ Por otro lado, en ausencia de $\mathrm{O}_{2}$ no se observa reacción en ninguna de las dos condiciones de $\mathrm{pH}$.

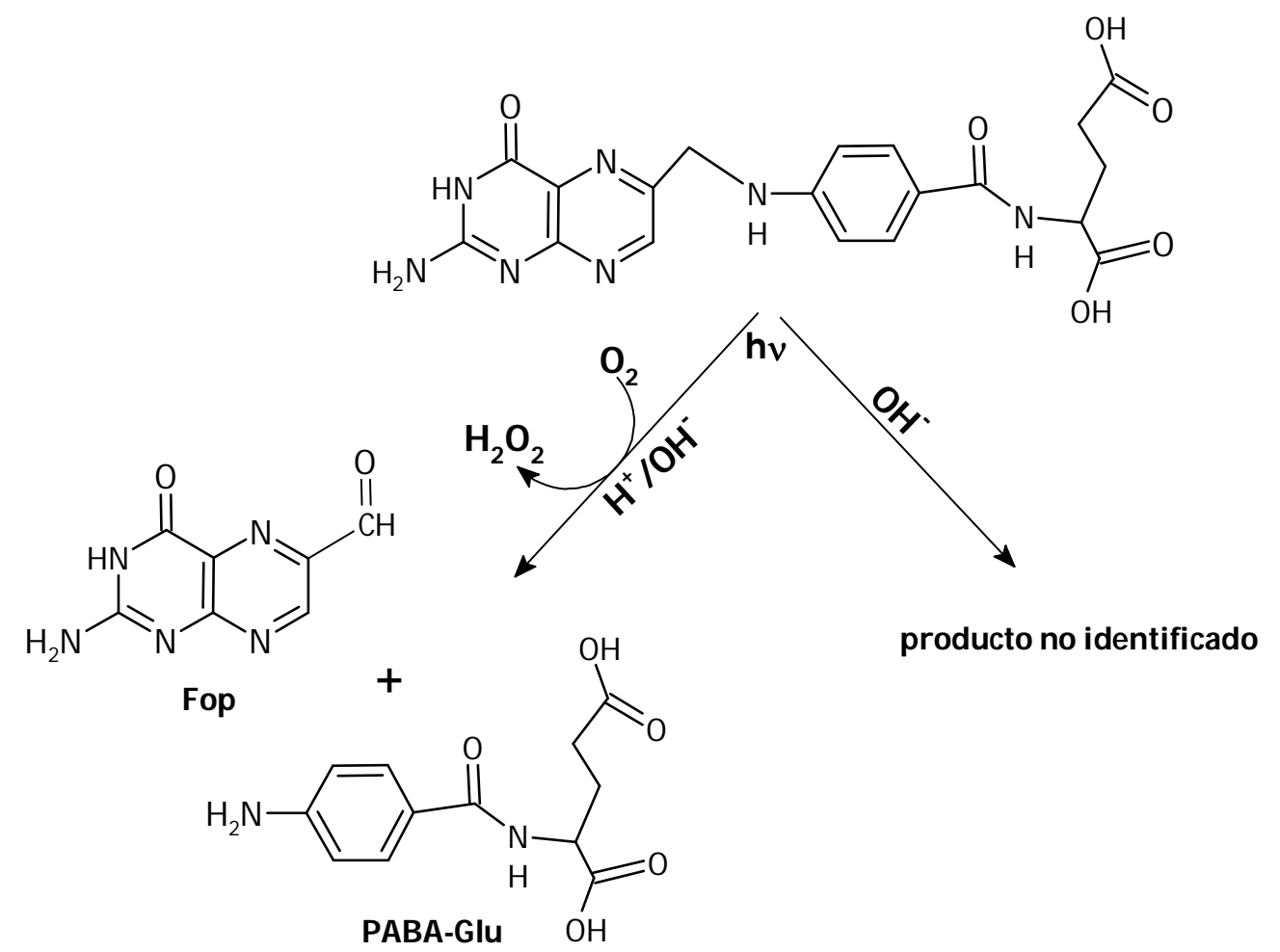

Esquema 3.3 Fotooxidación de PteGlu en solución acuosa.

\subsection{Reactividad fotoquímica de los derivados reducidos}

No existe demasiada información acerca de la fotoquímica de los dihidro y tetrahidroderivados. Una de las pocas reacciones de este tipo de pterinas que han sido descriptas en la literatura, es la reacción fotoquímica de 5,6,7,8-tetrahidrobiopterina ( $\mathrm{H}_{4} \mathrm{Bip}$ ), que posee una marcada dependencia con la longitud de onda de excitación. Bajo irradiación UV-B y en condiciones de $\mathrm{pH}$ fisiológico, $\mathrm{H}_{4} \mathrm{Bip}$ se fotooxida generando como único producto 7,8-dihidroxantopterina ( $\mathrm{H}_{2} \mathrm{Xap}$ ). Sin embargo, las soluciones acuosas aireadas de este tetrahidroderivado son fotoestables cuando se las expone a la radiación UV-A. ${ }^{18}$

Otro derivado pterínico reducido que ha sido estudiado es sepiapterina (Sep). Al contrario de lo observado para $\mathrm{H}_{4} \mathrm{Bip}$, la fotoquímica de Sep es independiente de la longitud de onda de irradiación. En soluciones aireadas irradiadas tanto con UV-A como con UV-B, Sep se oxida generando Cap y $\mathrm{H}_{2} \mathrm{O}_{2}{ }^{19}$. Por otra parte, cuando la radiación incide sobre soluciones anaeróbicas de Sep, tiene lugar la formación de un intermediario que luego, en contacto con $\mathrm{O}_{2}$, genera Cap y $\mathrm{H}_{2} \mathrm{O}_{2}{ }^{20}$ 
Cuando se expone a radiación visible $(438 \mathrm{~nm})$ una solución aireada de 6 -formil7,8-dihidropterina ( $\mathrm{H}_{2} \mathrm{Fop}$ ), ésta sufre una fotooxidación en el anillo pterínico, generando su análogo oxidado Fop y $\mathrm{H}_{2} \mathrm{O}_{2}$, con un rendimiento cuántico de consumo de reactivo de 0,0113 $\pm 0,0009$. Por otro lado, cuando se irradia $\mathrm{H}_{2} \mathrm{Fop}$ en ausencia de $\mathrm{O}_{2}$, se observa la formación de un intermediario que luego de airearse la solución, se transforma en Fop (Esquema 3.4).21 Este intermediario tiene características similares a los encontrados cuando se irradian en anaerobiosis las pterinas aromáticas mencionadas anteriormente, con lo cual podría ser un 5,8 dihidroderivado.

Por otro lado, cuando se irradia una solución aireada de $\mathrm{H}_{2}$ Xap con $\lambda_{\operatorname{exc}}=312 \mathrm{~nm}$ a pH fisiológico, se observa un muy bajo consumo del reactivo, con un rendimiento cuántico similar a los de Ptr o Mep. ${ }^{10}$ Este resultado es importante ya que, como se dijo anteriormente, $\mathrm{H}_{2}$ Xap es el producto de oxidación de $\mathrm{H}_{2} \mathrm{Bip}$ por $\mathrm{O}_{2} \mathrm{y}_{2} \mathrm{O}_{2}$, con lo cual, cuando se acumula $\mathrm{H}_{2}$ Bip en los tejidos de pacientes con vitíligo, este se oxidaría a $\mathrm{H}_{2}$ Xap, generando así un compuesto fotoestable con capacidad para reaccionar con especies oxidantes como $\mathrm{H}_{2} \mathrm{O}_{2} \mathrm{y}^{1} \mathrm{O}_{2}$.<smiles>Nc1nc2c(c(=O)[nH]1)N=C(C=O)C(C=O)N2C(=O)O</smiles>

\section{Esquema 3.4 Mecanismo de fotooxidación de $\mathrm{H}_{2}$ Fop.}

\subsection{Referencias}

1. A. H. Thomas, C. Lorente, A. L. Capparelli, M. R. Pokhrel, A. M. Braun and E. Oliveros, Fluorescence of pterin, 6-formylpterin, 6-carboxypterin and folic acid in aqueous solution: $\mathrm{pH}$ effects, Photochemical and Photobiological Sciences, 2002, 1, 421-426.

2. F. M. Cabrerizo, G. Petroselli, C. Lorente, A. L. Capparelli, A. H. Thomas, A. M. Braun and E. Oliveros, Substituent effects on the photophysical properties of pterin derivatives in acidic and alkaline aqueous solutions, Photochemistry and Photobiology, 2005, 81, 1234-1240.

3. C. Lorente, A. L. Capparelli, A. H. Thomas, A. M. Braun and E. Oliveros, Quenching of the fluorescence of pterin derivatives by anions, Photochemical and Photobiological Sciences, 2004, 3, 167-173.

4. M. P. Serrano, M. Vignoni, M. L. Dántola, E. Oliveros, C. Lorente and A. H. Thomas, Emission properties of dihydropterins in aqueous solutions, Physical Chemistry Chemical Physics, 2011, 13, 7419-7425.

5. A. H. Thomas, C. Lorente, A. L. Capparelli, C. G. Martínez, A. M. Braun and E. Oliveros, Singlet oxygen $\left({ }^{1} \Delta_{\mathrm{g}}\right)$ production by pterin derivatives in aqueous solutions, Photochemical and Photobiological Sciences, 2003, 2, 245-250.

6. F. M. Cabrerizo, A. H. Thomas, C. Lorente, M. L. Dántola, G. Petroselli, R. Erra-Balsells and A. L. Capparelli, Generation of Reactive Oxygen Species during the Photolysis of 6-(Hydroxymethyl)pterin in Alkaline Aqueous Solutions, Helvetica Chimica Acta, 2004, 87, 349-365. 
7. A. H. Thomas, R. Cabrerizo, M. Vignoni, R. Erra-Balsells, F. M. Cabrerizo and A. L. Capparelli, Photoinduced formation of reactive oxygen species from the acid form of 6-(hydroxymethyl)pterin in aqueous solution, Helvetica Chimica Acta, 2006, 89, 1090-1104.

8. C. Chahidi, M. Aubailly, A. Momzikoff, M. Bazin and R. Santus, Photophysical and photosensitizing propierties of 2-amino-4 pteridinone: a natural pigment, Photochemistry and Photobiology, 1981, 33, 641-649.

9. J. W. Ledbetter, W. Pfleiderer and J. H. Freisheim, Photosensitized reduction of l-biopterin in the active ternary complex of dihydrofolate reductase, Photochemistry and Photobiology, 1995, 62, 71-81.

10. M. L. Dántola, Reactividad térmica y fotoquímica de 7,8-dihidropterinas frente a especies oxidantes en condiciones fisiológicas, in Departamento de Química - Facultad de Ciencias Exactas, Universidad Nacional de La Plata, La Plata, 2008, p. 250.--

11. G. Suárez, F. M. Cabrerizo, C. Lorente, A. H. Thomas and A. L. Capparelli, Study of the photolysis of 6carboxypterin in acid and alkaline aqueous solutions, Journal of Photochemistry and Photobiology A: Chemistry, 2000, 132, 53-57.

12. F. M. Cabrerizo, C. Lorente, M. Vignoni, R. Cabrerizo, A. H. Thomas and A. L. Capparelli, Photochemical behavior of 6-methylpterin in aqueous solutions: Generation of reactive oxygen species, Photochemistry and Photobiology, 2005, 81, 793-801.

13. A. H. Thomas, G. Suárez, F. M. Cabrerizo, R. Martino and A. L. Capparelli, Study of the photolysis of folic acid and 6-formylpterin in acid aqueous solutions, Journal of Photochemistry and Photobiology A: Chemistry, 2000, 135, 147-154.

14. A. H. Thomas, G. Suárez, F. M. Cabrerizo and A. L. Capparelli, Photochemistry of 6-formylpterin in alkaline medium, Helvetica Chimica Acta, 2001, 84, 3849-3860.

15. A. H. Thomas, G. Suárez, F. M. Cabrerizo, F. S. García Einschlag, R. Martino, C. Baiocchi, E. Pramauro and A. L. Capparelli, Photochemical behavior of folic acid in alkaline aqueous solutions and evolution of its photoproducts, Helvetica Chimica Acta, 2002, 85, 2300-2315.

16. R. Mengel, W. Pfleiderer and W. R. Knappe, Norrish type II analogous photodegradation of neopterin and biopterin to 5,8 -dihydropterin-6-aldehyde, Tetrahedron Lett., 1977, 18, 2817-2820.

17. M. L. Dántola, M. P. Denofrio, B. Zurbano, C. S. Gimenez, P. R. Ogilby, C. Lorente and A. H. Thomas, Mechanism of photooxidation of folic acid sensitized by unconjugated pterins, Photochemical and Photobiological Sciences, 2010, 9, 1604-1612.

18. K. U. Schallreuter, J. M. Wood, C. Körner, K. M. Harle, V. Schulz-Douglas and E. R. Werner, 6Tetrahydrobiopterin functions as a UVB-light switch for de novo melanogenesis, Biochimica et Biophysica Acta (BBA) - Protein Structure and Molecular Enzymology, 1998, 1382, 339-344.

19. H. Rokos and K. Schallreuter, Photo-oxidation of sepiapterin produces pterin-6-carbolxylic acid and $\mathrm{H}_{2} \mathrm{O}_{2}$ in vitro, in Chemistry and biology of pteridines and folates, ed. S. Milstien, G. Kapatos, R. A. Levine and B. Shane, Kluwe Academic, 2002, pp. 55-59.

20. H. Rokos, W. D. Beazley and K. U. Schallreuter, Oxidative stress in vitiligo: Photo-oxidation of pterins produces $\mathrm{H}_{2} \mathrm{O}_{2}$ and pterin-6-carboxylic acid, Biochem. Biophys. Res. Commun., 2002, 292, 805-811.

21. M. L. Dántola, A. H. Thomas, E. Oliveros and C. Lorente, Visible-light photochemistry of 6-formyl-7,8dihydropterin in aqueous solution, Journal of Photochemistry and Photobiology A: Chemistry, 2010, 209, 104-110. 
40 Capítulo 3 


\section{PARTE II}

\section{MATERIALES Y MÉTODOS EXPERIMENTALES}





\section{Capítulo 4}

\section{Preparación de soluciones y metodología de trabajo}

\subsection{Diseño general de los experimentos}

En este trabajo de tesis se realizaron principalmente dos tipos de experimentos. Por un lado, se estudiaron distintas reacciones fotoquímicas mediante irradiaciones continuas. La irradiación o fotólisis continua implica la iluminación de una muestra con radiación procedente de una lámpara de intensidad constante y por períodos de tiempo relativamente largos. La duración es en referencia a los tiempos de técnicas como fotólisis de flash, donde el pulso de luz es del orden de micro, mili o nanosegundos.

Para los experimentos de fotólisis se empleó la metodología siguiente: primero se prepararon las soluciones, siguiendo el protocolo descripto en la siguiente sección. A modo de control, a cada solución se le registró el correspondiente espectro de absorción. Luego, se irradiaron las muestras por diferentes períodos de tiempo, y se estudiaron las soluciones irradiadas analizando consumo de reactivo, generación de productos e intermediarios y producción de distintas especies reactivas. Ésto se realizó utilizando diversas técnicas analíticas que se describirán en el siguiente capítulo. Por último, se analizaron los resultados con el fin de calcular parámetros cinéticos y fotoquímicos, y dilucidar mecanismos de reacción.

Por otra parte, se realizaron estudios de estabilidad de los productos generados durante las distintas reacciones fotoquímicas. Para ésto, se utilizó la siguiente metodología: las soluciones se irradiaron durante un determinado intervalo de tiempo e inmediatamente después de interrumpir la irradiación, se mantuvieron en oscuridad a una temperatura y condición de $\mathrm{O}_{2}$ (presencia o ausencia) determinada. Al período de tiempo transcurrido en estas condiciones se lo denominó, arbitrariamente, tiempo de oscuridad. Entonces, a distintos tiempos de oscuridad se estudiaron las soluciones con diferentes técnicas, analizando consumo de fotoproducto y generación de otros productos, así como también la generación de especies reactivas. Luego, se analizaron los resultados para esclarecer mecanismos de reacción. 
El siguiente esquema resume el diseño experimental seguido en este trabajo de tesis descripto en los párrafos anteriores:

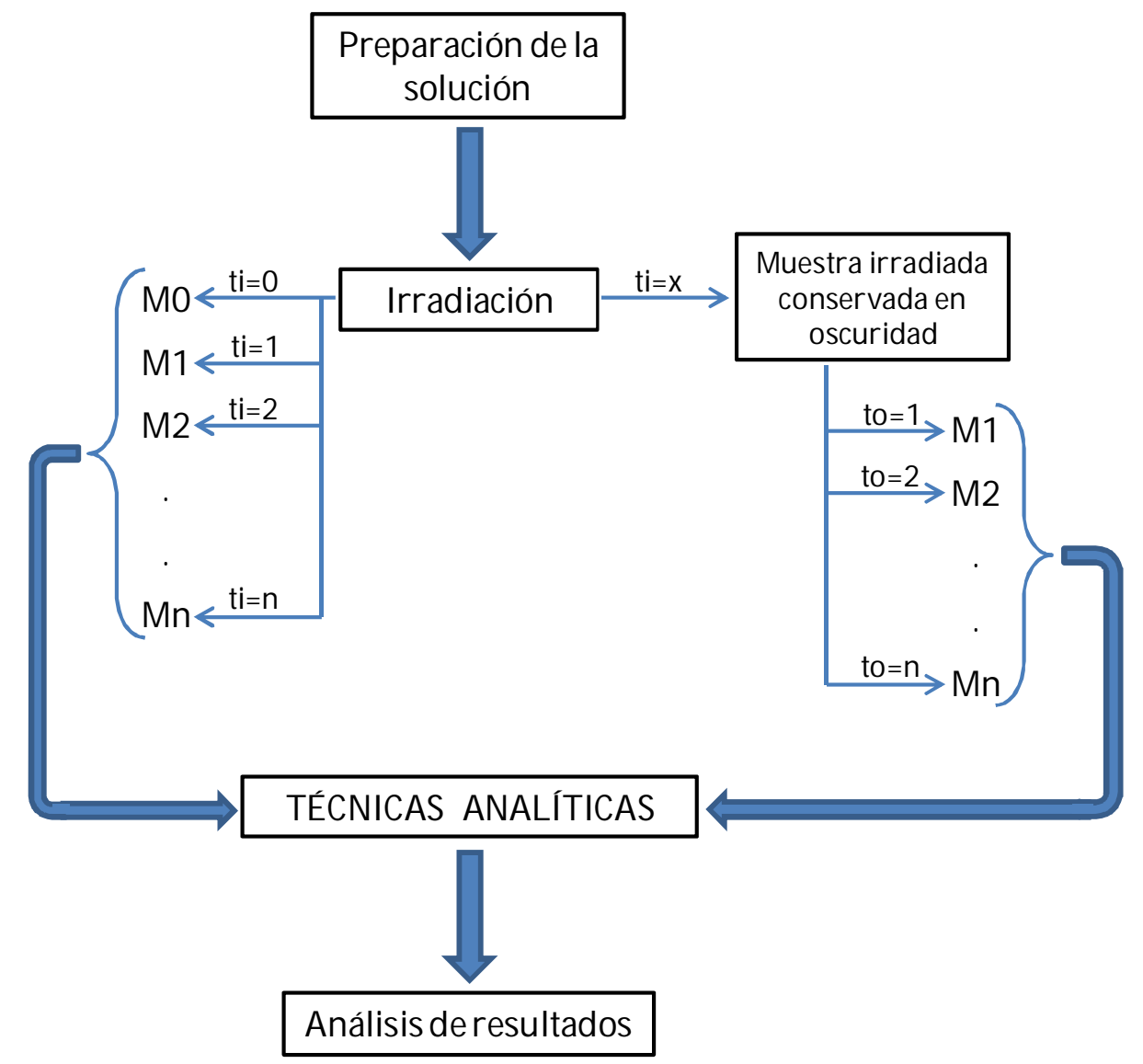

Esquema 4.1 Diseño experimental utilizado en este trabajo de tesis.

\subsection{Reactivos y preparación de soluciones de pterinas}

\subsubsection{Reactivos}

Las pterinas oxidadas y 7,8-dihidropterinas utilizadas en este trabajo de tesis fueron sintetizadas y provistas por el Laboratorio Schircks (Suiza). Todas fueron usadas sin posterior purificación, debido a que presentan una pureza mayor al 99\%. En particular, los compuestos usados en este trabajo fueron: pterina (Ptr), biopterina (Bip), neopterina (Nep), 6-hidroximetilpterina ( $\mathrm{Hmp}$ ), 6-formilpterina (Fop), 6-carboxipterina (Cap), 6-metilpterina (Mep), 7,8-dihidrobiopterina ( $\left.\mathrm{H}_{2} \mathrm{Bip}\right)$, 7,8-dihidroneopterina ( $\left.\mathrm{H}_{2} \mathrm{Nep}\right)$ y 7,8-dihidroxantopterina ( $\left.\mathrm{H}_{2} \mathrm{Xap}\right)$.

A continuación se listan otros reactivos utilizados: $\mathrm{NaOH}, \mathrm{HCl}, \mathrm{H}_{2} \mathrm{O}_{2}$ (30 \%), (Merck), $\mathrm{D}_{2} \mathrm{O}$ (>99,9 \%, Sigma-Aldrich), hidróxido de sodio deuterado (NaOD, CEA, > 99 \%) y ácido clorhídrico deuterado ( $\mathrm{DCl}$, Aldrich, 99,5 \%). Tris-hidroximetil-aminometano clorhídrico (Trizma, $\left.\mathrm{NH}_{2} \mathrm{C}\left(\mathrm{CH}_{2} \mathrm{OH}\right)_{3} \cdot \mathrm{HCl}\right)$ y acetato de amonio $\left(\mathrm{CH}_{3} \mathrm{COONH}_{4}\right.$ ) (Sigma- 
Aldrich); fosfato diácido de potasio $\left(\mathrm{KH}_{2} \mathrm{PO}_{4}\right)$ (Anedra) e ioduro de potasio (IK) (Laboratorios Cicarelli). Metanol y acetonitrilo calidad HPLC (J. T. Baker). Además, se empleó superóxido dismutasa de eritrocito bovino (SOD), citocromo c de corazón equino (Cyt), 5,5-dimethil-1-pirrolin-N-óxido (DMPO) (Sigma-Aldrich).

El agua utilizada para preparar las distintas soluciones fue de calidad milliQ (agua destilada purificada en un equipo Milli Q Reagent Water System (resistividad $\sim 10 \mathrm{M} \Omega$ $\left.\mathrm{cm}^{-1}\right)$ ).

\subsubsection{Soluciones acuosas}

En general, las pterinas reducidas son más inestables que los derivados aromáticos cuando se encuentran en soluciones acuosas aireadas a temperatura ambiente. ${ }^{1}$ La oxidación de $\mathrm{H}_{2}$ Bip y $\mathrm{H}_{2} \mathrm{Nep}$ genera, principalmente, $\mathrm{H}_{2} \mathrm{Xap}$, sin embargo la velocidad de esta reacción es diferente para ambos dihidroderivados. Mientras que $\mathrm{H}_{2}$ Bip tiene un tiempo de vida media $\left(\mathrm{t}_{1 / 2}\right)$ de $\approx 1700 \mathrm{~h}, \mathrm{H}_{2} \mathrm{Nep}$ es más inestable, con un $\mathrm{t}_{1 / 2}=60 \mathrm{~h}$ (Sección 2.3). En consecuencia, la degradación que sufren estos dihidroderivados durante la preparación de las soluciones es despreciable. No obstante, las soluciones de $\mathrm{H}_{2} \mathrm{Nep}$ fueron utilizadas dentro de las $12 \mathrm{~h}$ después de su preparación, mientras que las soluciones de $\mathrm{H}_{2}$ Bip pudieron utilizarse hasta 48 - $72 \mathrm{~h}$ posteriores a la preparación.

Para preparar soluciones de pterinas aromáticas o dihidroderivados se disolvió el sólido en agua libre de iones metálicos. Se agregaron pequeños volúmenes (10 - $100 \mu \mathrm{l})$ de $\mathrm{NaOH}$ 0,5 - 1 M para facilitar la disolución, ya que las pterinas presentan grupos ionizados en medio alcalino. El sistema se mantuvo en agitación por alrededor de $1 \mathrm{~h}$ hasta lograr la completa disolución del reactivo. Luego, mediante el uso de matraces, se llevó la solución a volumen final y se transvasó a frascos de color caramelo para evitar la degradación que pudieran sufrir estos derivados pterínicos por exposición a la luz.

El pH de trabajo dependió del compuesto utilizado y de cada experimento en particular. Para las pterinas aromáticas se trabajó, generalmente, en dos condiciones de pH: 5,5 y 10,5. De esta manera, en las soluciones de trabajo sólo se encontró presente una única especie ácido-base del compuesto en estudio ya que, como se explicó en el Capítulo 2, el pKa de la mayoría de las pterinas aromáticas es cercano a 8. En el caso de las pterinas reducidas, se trabajó en condiciones de pH comprendidas entre 7,0 y 7,4, 
condición en la cual se encuentra presente únicamente la especie neutra de estos compuestos que presentan valores de $\mathrm{pK}_{\mathrm{a}}$ cercanos a 10.

Las soluciones de ácido y álcali utilizadas para llevar a pH se prepararon a partir de una solución concentrada del reactivo comercial, en el caso del $\mathrm{HCl}$ y, por pesada de granallas, en el caso del $\mathrm{NaOH}$. Las concentraciones variaron entre 0,1 y 2 M. Las medidas de pH se realizaron en un pH-metro PHM22O (Radiometer Copenhagen) en combinación con un electrodo de pHC3001-9 (Radiometer Analytical). La calibración de los equipos se realizó empleando soluciones amortiguadoras comerciales con valores de pH 4,00; 7,00 y 10,00. Es necesario destacar que no se utilizaron soluciones reguladoras para ajustar el pH de las soluciones debido a que se sabe que ciertos aniones, como por ejemplo fosfato y acetato, son capaces de desactivar los estados excitados de las pterinas. $^{2}$

Con respecto a las concentraciones de las soluciones de pterinas estudiadas, las mismas variaron, generalmente, entre 50 y $200 \mu \mathrm{M}$. Para determinar la concentración exacta de las soluciones empleadas en los distintos experimentos se utilizaron distintos métodos: a) a partir de la cantidad de sólido pesado, el volumen de solución y el peso molecular del compuesto; b) a partir del factor de dilución y la concentración (calculada según a) de una solución más concentrada y c) a partir de medidas de absorbancia y los correspondientes valores de los coeficientes de absorción molar $(\varepsilon) .^{3}$

\subsubsection{Soluciones en agua deuterada}

Para las medidas de Resonancia Magnética Nuclear (RMN) se utilizó agua deuterada $\left(\mathrm{D}_{2} \mathrm{O}\right)$ como solvente. Las concentraciones de pterinas variaron entre 0,7 y 2 $\mathrm{mM}$, siendo más elevadas que en $\mathrm{H}_{2} \mathrm{O}$ debido a su mayor solubilidad en el solvente deuterado. Se partió de $\mathrm{D}_{2} \mathrm{O}$ previamente alcalinizada a $\mathrm{pD} \sim 11$ a la cual se agregó el sólido a disolver y se mantuvo bajo agitación magnética por un período de 45 a 60 min, sellando los envases con parafilm, para evitar el intercambio de $\mathrm{D}_{2} \mathrm{O}$ con $\mathrm{H}_{2} \mathrm{O}$ proveniente de la humedad ambiente. Luego, las soluciones se traspasaron a envases color caramelo para su disposición final y almacenamiento. La concentración se determinó mediante el cálculo del sólido pesado y el volumen de $\mathrm{D}_{2} \mathrm{O}$ agregado.

El pD (=-log $\left.\left[\mathrm{D}^{+}\right]\right)$fue medido con el pH-metro previamente nombrado y los valores de pD se calcularon aplicando la siguiente corrección a cada medida4: 


$$
\mathrm{pD}=\mathrm{pH}+0,4
$$

donde $\mathrm{pH}$ es el valor leído en el instrumento. El valor final de pD se alcanzó mediante el agregado con micropipeta de pequeños volúmenes de soluciones relativamente concentradas de $\mathrm{NaOD}$ y $\mathrm{DCl}$ en $\mathrm{D}_{2} \mathrm{O}$.

\subsubsection{Condiciones anaeróbicas y saturación de $\mathrm{O}_{2}$}

Para realizar ciertos experimentos fue necesario eliminar el $\mathrm{O}_{2}$ disuelto en la solución. Para tal fin, se prosiguió de la siguiente manera: mililitros de solución preparada tal como se mencionó anteriormente, se colocaron en celdas de cuarzo con dimensiones de $1 \mathrm{~cm}$ x $1 \mathrm{~cm}$ o de $1 \mathrm{~cm} \mathrm{x} \mathrm{0,4} \mathrm{cm,} \mathrm{con} \mathrm{tapa} \mathrm{a} \mathrm{rosca} \mathrm{y} \mathrm{septum} \mathrm{de} \mathrm{silicona.} \mathrm{Se}$ insertaron dos agujas atravesando el septum superior, una de ellas se introdujo hasta el fondo de la celda y operó como vía de entrada del gas ( $\mathrm{Ar}$ o $\mathrm{N}_{2}$ ), mientras que la otra se dejó por encima de la superficie de la solución actuando como vía de salida. Así, se burbujearon las soluciones con el gas inerte durante aproximadamente 20 minutos. La disposición de las agujas permite que la presión alcanzada en el interior de la celda sea idéntica a la presión externa (atmosférica), evitando entonces que se genere una condición de sobre-presión en el interior de la celda. Luego, se extrajeron las agujas y se cubrió la parte superior de la celda con parafilm, para evitar el intercambio de gases y la consecuente pérdida de anaerobiosis.

Es necesario aclarar que durante el burbujeo de Ar, además de $\mathrm{O}_{2}$ también se elimina el $\mathrm{CO}_{2}$ presente en la solución, el cual está en equilibrio con $\mathrm{H}_{2} \mathrm{CO}_{3}$ (ácido débil que, en solución acuosa, se encuentra parcialmente disociado) (reacciones 1 a 3). Consecuentemente, cuando se elimina el $\mathrm{CO}_{2}$, los equilibrios de las reacciones 1 a 3 se desplazan hacia la izquierda, disminuyendo así la concentración de protones, con la consecuente alcalinización de la solución. Por lo tanto, se tomaron los espectros de absorción de las soluciones antes y después de burbujear el gas inerte, para asegurar la conservación de la forma ácido-base del derivado pterínico deseada. El espectro también sirve para controlar que no se haya evaporado el solvente y concentrado la solución, ya que un burbujeo por tiempos prolongados puede provocar la pérdida de cantidades substanciales de solvente por arrastre de moléculas de $\mathrm{H}_{2} \mathrm{O}$ con la corriente de gas (los gases inertes utilizados están libres de $\mathrm{H}_{2} \mathrm{O}$ ). 


$$
\begin{aligned}
\mathrm{CO}_{2}(\mathrm{~g}) & \rightleftarrows \mathrm{CO}_{2}(\mathrm{aq}) \\
\mathrm{CO}_{2}(\mathrm{aq})+\mathrm{H}_{2} \mathrm{O} & \rightleftarrows \mathrm{H}_{2} \mathrm{CO}_{3} \\
\mathrm{H}_{2} \mathrm{CO}_{3} & \rightleftarrows \mathrm{HCO}_{3}{ }^{-}+\mathrm{H}^{+}
\end{aligned}
$$

En el caso de los experimentos de RMN, por cuestiones de tamaño y geometría de los tubos utilizados, se modificó ligeramente el procedimiento de burbujeo y eliminación de $\mathrm{O}_{2}$ disuelto. Concretamente, las soluciones se burbujearon con $\mathrm{N}_{2}$ directamente en los tubos de RMN, insertando una única aguja (actuando de canal de ingreso de $\mathrm{N}_{2}$ ) y dejando levemente abierta la tapa del tubo para eliminar el aire como vía de escape 0 salida del gas.

Por otro lado, para estudiar el efecto de la variación de la concentración de $\mathrm{O}_{2}$ en diversos procesos, algunos experimentos se realizaron en soluciones saturadas en $\mathrm{O}_{2}$. En estas condiciones la concentración de $\mathrm{O}_{2}$ es, aproximadamente, 5 veces superior a la de las soluciones equilibradas en aire. Para esto, se procedió al burbujeo de dicho gas de la misma manera que se realizó con el Ar mencionado en los párrafos previos.

\subsection{Irradiación de soluciones}

Las soluciones fueron irradiadas con dos fuentes distintas:

- Sistema de irradiación I: lámparas Rayonet RPR (Southern N.E. Ultraviolet Co.) que presentan un máximo de emisión en 350 y un ancho de banda de alrededor de 20 nm.

- Sistema de irradiación II: lámpara de Xe/ Hg Osram de 1000W, cuyo haz de luz, antes de incidir en la celda que contiene la muestra, se hace pasar por un filtro de agua y un monocromador (ISA Jobin-Yvon B204) de $6 \mathrm{~nm}$ de ancho de banda. Se utilizaron distintas longitudes de onda de excitación de la muestra $\left(\lambda_{\text {exc }}\right)$, según el reactivo a irradiar y el espectro de emisión de la lámpara. Por ejemplo, se utilizó una $\lambda_{\text {exc }}$ de $335 \mathrm{~nm}$ cuando se trabajó con los dihidroderivados, mientras que, una mezcla de Bip y $\mathrm{H}_{2}$ Bip se irradió a $380 \mathrm{~nm}$, condición experimental que permite excitar selectivamente el compuesto aromático Bip.

En el Esquema 4.2 se muestran los sistemas de irradiación utilizados con la geometría específica empleada. Exceptuando los experimentos de RMN, donde las soluciones se irradiaron directamente en los tubos de RMN, en general, se utilizaron 
celdas de cuarzo Hellma de dimensiones $1 \mathrm{~cm} \times 1 \mathrm{~cm}$ o de $1 \mathrm{~cm}$ x 0,4 cm. Estas celdas tienen una capacidad volumétrica de 3 y 1,4 ml, respectivamente. Las soluciones se homogeneizaron con un agitador magnético durante todo el tiempo de fotólisis, evitando así la acumulación de fotoproductos en la cara de la celda más próxima a la lámpara.

a)

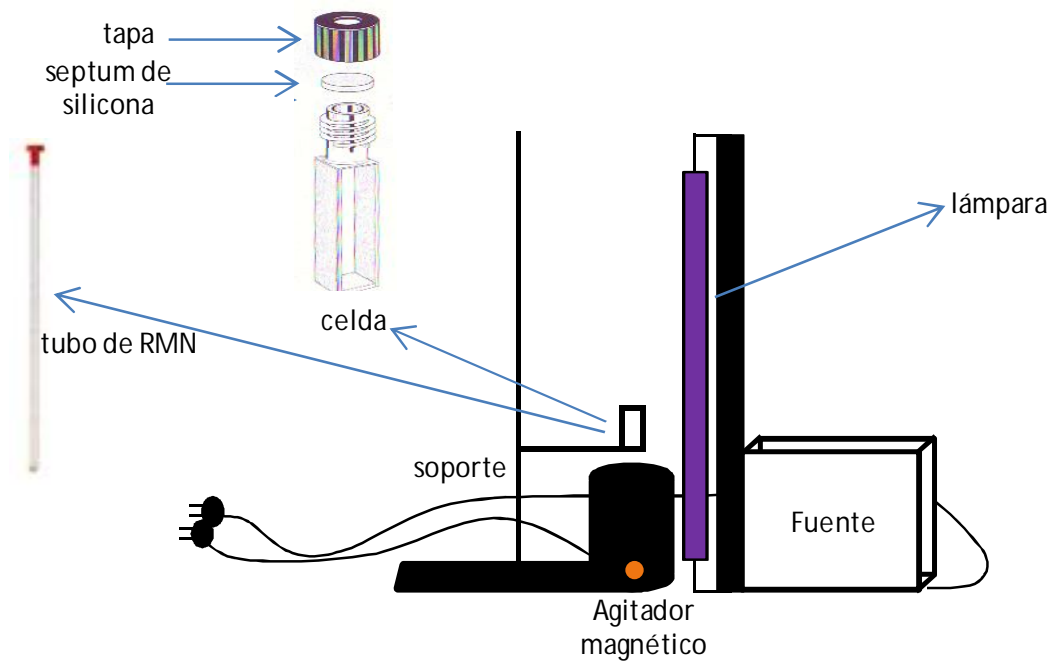

b)
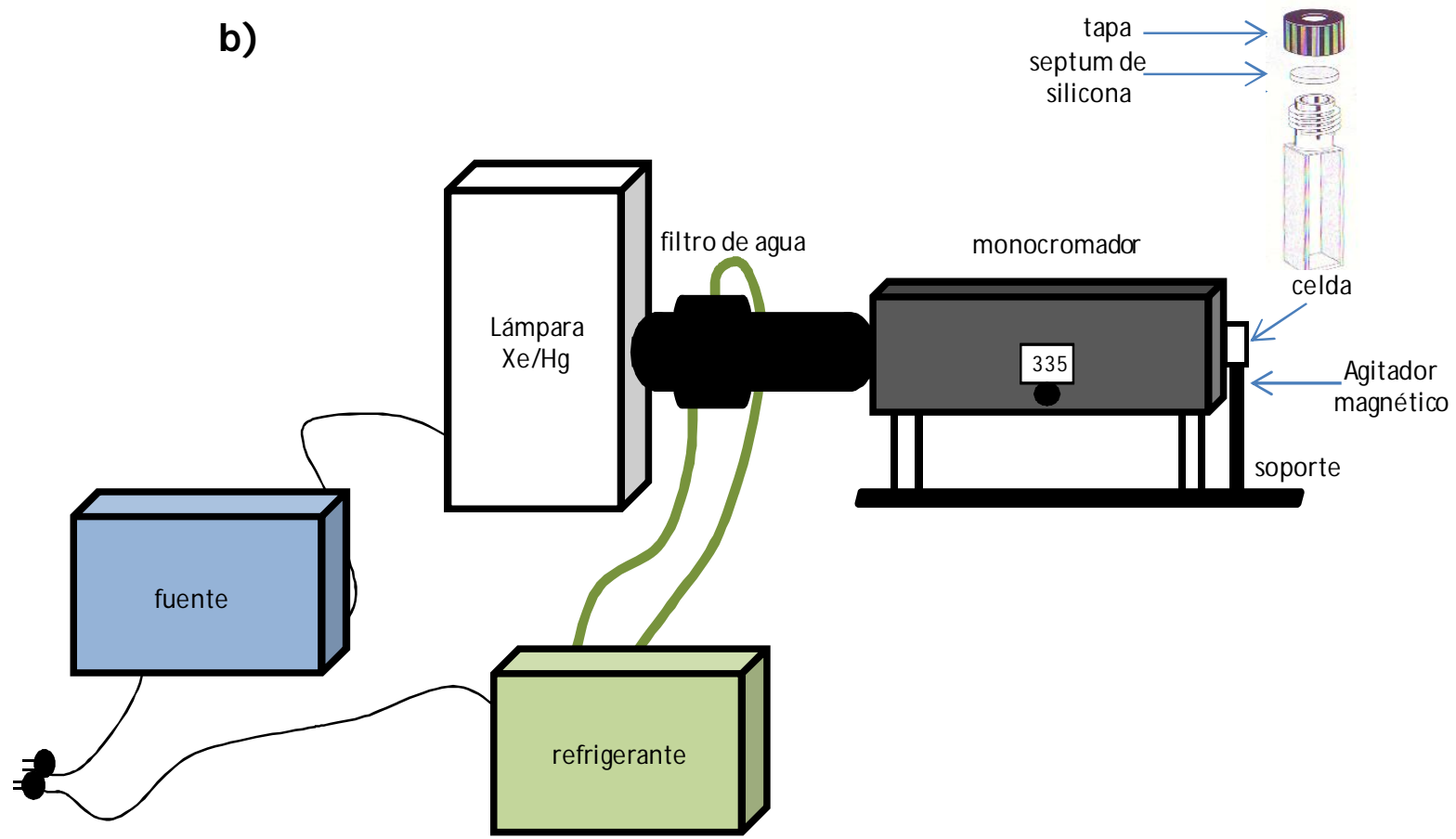

Esquema 4.2 Esquema de los dos sistemas de irradiación utilizados. a) Sistema de irradiación I, b) Sistema de irradiación II.

En cada experimento de fotólisis se extrajeron muestras a distintos tiempos para su posterior análisis. En la mayoría de los casos, las soluciones irradiadas se analizaron con técnicas "destructivas" que modifican su composición o implican un consumo neto de la misma (detección de $\mathrm{H}_{2} \mathrm{O}_{2}, \mathrm{HPLC}$ ). En estos casos, se introdujo en la celda una cantidad determinada de solución, se irradió y luego se retiró por completo la totalidad 
del volumen para su análisis. A continuación, para estudiar la reacción en otro tiempo de irradiación, se volvió a cargar la celda con solución madre y se irradió nuevamente.

En otros casos, se utilizaron técnicas de análisis "no destructivas", por ejemplo, cuando sólo fue necesario observar los cambios espectrales o cuando se determinó el consumo de $\mathrm{O}_{2}$. En estos casos, se irradió la solución un tiempo dado, luego se tomó el espectro y, posteriormente, se continuó irradiando la misma solución, hasta finalizar el experimento.

\subsection{Determinación de rendimientos cuánticos}

Si se tiene una única especie capaz de absorber la radiación, la ley de LambertBeer se puede expresar de la siguiente manera:

$$
q_{p, \lambda}=q_{p, \lambda}^{0}\left(1-10^{-\varepsilon(\lambda) l C}\right)
$$

donde $\mathrm{q}_{p, \lambda}$ es la intensidad de la luz absorbida por el soluto en la solución con una concentración molar $\mathrm{C}$, a una longitud de onda $\lambda$, l es el camino óptico $y, \mathrm{q}^{0} \mathrm{p}, \lambda \mathrm{y} \varepsilon(\lambda)$ son la intensidad de la luz incidente y el coeficiente de absorción molar a la longitud de onda $\lambda$. $q_{p, \lambda} \mathrm{y} \mathrm{q}^{0} \mathrm{p}, \lambda$ se expresan en fotones por unidad de tiempo.

$\mathrm{q}^{\mathrm{v}} \mathrm{p}, \lambda$ representa el número de fotones por unidad de tiempo y por unidad de volumen que son absorbidos por una muestra. Tomando $\mathrm{V}$ como el volumen de la solución, entonces la relación entre $\mathrm{q}_{\mathrm{p}, \lambda}^{\mathrm{v}} \mathrm{y} \mathrm{q}_{\mathrm{p}, \lambda}$ es:

$$
\mathbf{q}_{\mathbf{p}, \lambda}^{\mathbf{v}}=\mathbf{q}_{\mathbf{p}, \lambda} / \mathbf{V}
$$

Se define el rendimiento cuántico de una reacción fotoquímica como el cociente entre el número de moléculas que reaccionan y el número de fotones absorbidos. Este parámetro será representado por la letra griega “ $\Phi$ ”.

$$
\Phi_{(\lambda) R}=\frac{-\mathbf{d}[\mathbf{R}] / \mathbf{d t}}{\mathbf{q}_{\mathbf{p}, \lambda}^{\mathbf{v}}} \quad \Phi_{(\lambda) \mathrm{P}}=\frac{\mathbf{d}[\mathbf{P}] / \mathbf{d t}}{\mathbf{q}_{p, \lambda}^{\mathbf{v}}}
$$

(6)

Para calcular un rendimiento cuántico, basta con medir la velocidad de desaparición de un reactivo $(\mathrm{d}[\mathrm{R}] / \mathrm{dt})$ o la velocidad de formación de un producto $(\mathrm{d}[\mathrm{P}] / \mathrm{dt})$, las cuales pueden determinarse, por ejemplo, por HPLC. Además, se debe 
determinar el valor de $\mathrm{q}_{\mathrm{p}, \lambda}^{\mathrm{v}}$, para lo cual es necesario determinar el valor de $\mathrm{q}_{\mathrm{p}, \lambda}^{0} \circ \mathrm{q}^{0, \mathrm{v}_{\mathrm{p}, \lambda}}$ que se define como el número de moles de fotones (Einstein) que alcanzan la celda por unidad de tiempo y por unidad de volumen $\left(\mathrm{q}^{0} \mathrm{p}, \lambda / \mathrm{V}\right)$. Esto se realizó mediante una actinometría, la cual se describe en la siguiente sección. $\mathrm{q}^{0, \mathrm{v}} \mathrm{p}, \lambda$ depende siempre de factores geométricos, como el tamaño de la celda, la distancia comprendida entre ella a la lámpara, etc. Consecuentemente, para cada geometría de irradiación utilizada es necesario realizar una actinometría.

Existen dos condiciones de trabajo en las cuales se pueden determinar los rendimientos cuánticos:

1. Condición de absorción total de luz, sabiendo el $\varepsilon$ de la sustancia a irradiar a la $\lambda_{\text {exc }}$, se trabaja en condiciones tales que $\mathrm{A}=\varepsilon$ l C $\gg 1$, conduciendo a que $\mathrm{q}_{\mathrm{p}, \lambda} \cong \mathrm{q}_{\mathrm{p}, \lambda}^{0}$ (según

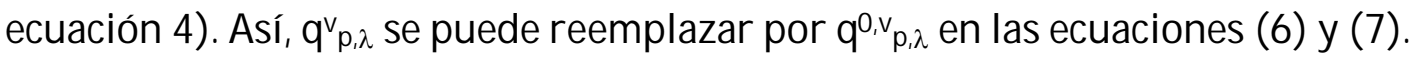

2. Condición en la que no se puede trabajar con absorción total de luz, por ende, se debe utilizar $\mathrm{q}_{\mathrm{p}, \lambda}^{\mathrm{v}}$ para determinar el valor de $\Phi$.

Al obtener las velocidades se deben cumplir dos condiciones: por un lado, la absorbancia del reactivo no debe cambiar significativamente en el tiempo de trabajo. Es decir, que la luz absorbida por unidad de tiempo debe permanecer aproximadamente constante. Por otra parte, la absorbancia de los productos a la $\lambda_{\text {exc }}$ debe ser despreciable con respecto a la del reactivo. De esta manera, la luz incidente es absorbida únicamente por el reactivo.

Cuando estas condiciones dejan de cumplirse, en general, a medida que avanza la reacción, se observa una disminución en la velocidad de consumo de reactivo. Aunque esto puede variar dependiendo del mecanismo de reacción del proceso en estudio. Para obtener estos rendimientos cuánticos se diseñó una geometría en la cual los intervalos de tiempo en los que se cumplen estas condiciones sean lo suficientemente largos como para realizar varias medidas de concentración.

\subsection{Actinometría}

Para determinar la intensidad de la radiación incidente en una celda de reacción $\left(\mathrm{q}^{0} \mathrm{p}, \lambda\right)$ necesaria para calcular el rendimiento cuántico, se utilizó Aberchrome 540 (Aberchromics Ltd.) ${ }^{5}$ como actinómetro químico. Este compuesto es el anhídrido del 
ácido (E) $\alpha$-(2,5-dimetil-3-furiletiliden) (isopropiliden) succínico (DFIS), que al ser irradiado con una longitud de onda entre 310 y $370 \mathrm{~nm}$ adquiere un color rojo intenso correspondiente al 7,7a-dihidro-2,4,7,7a-pentametilbenzo[b]furano (7,7a-DHBF). Este producto posee una banda de absorción entre 430 y $550 \mathrm{~nm}$ y, si es excitado en este intervalo, la reacción ocurre en sentido inverso. En la Figura 6.1 se exponen las estructuras químicas de las dos especies y sus respectivos espectros de absorción.
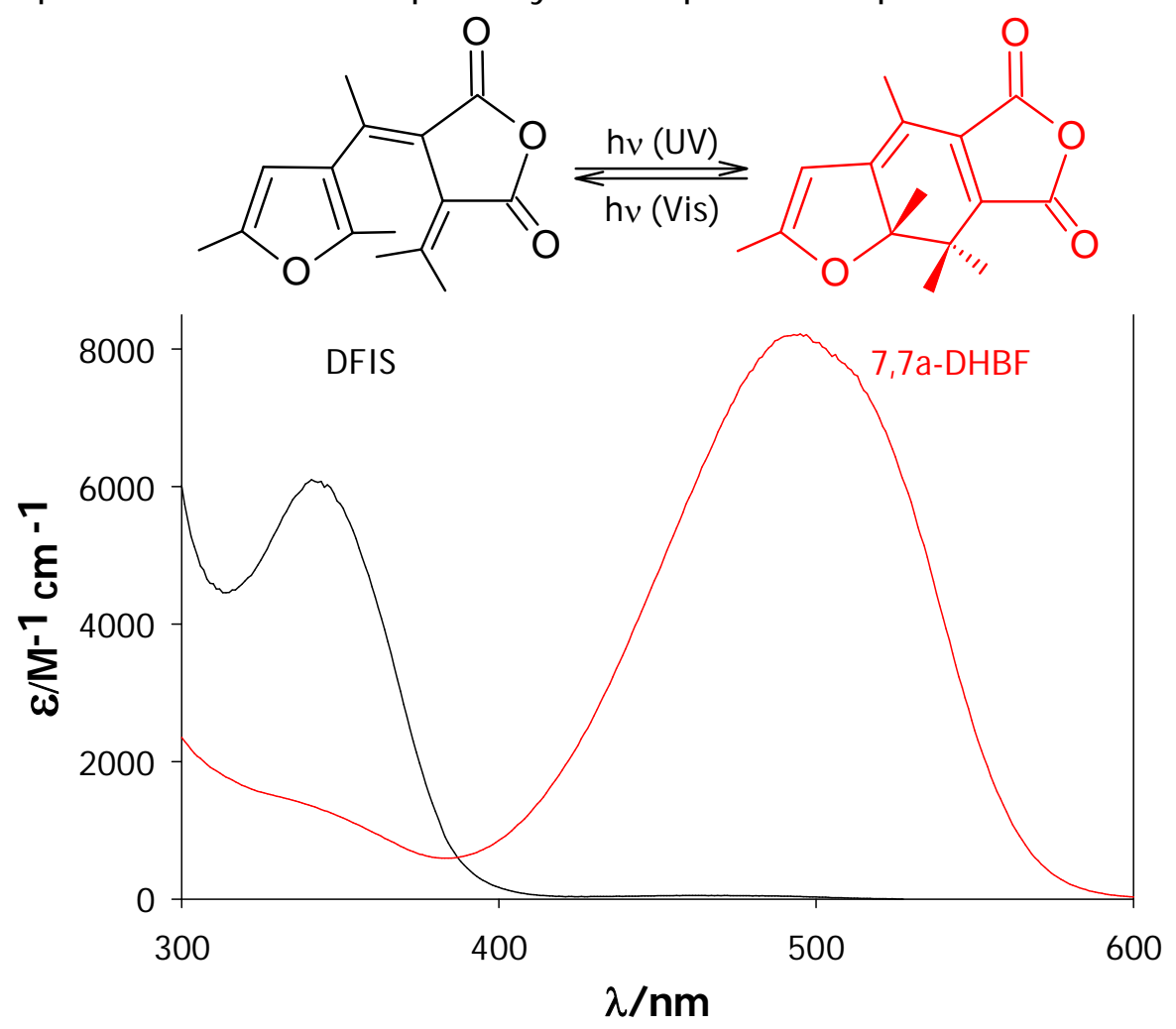

Figura 6.1 Estructura química y espectros de absorción en tolueno de los dos isómeros del Aberchrome 540.

El Aberchrome 540 puede utilizarse en distintos solventes, en este caso se utilizaron soluciones de tolueno. La $\lambda$ del máximo de absorción del DFIS no depende de la naturaleza del solvente $\left(\lambda_{\max }{ }^{\mathrm{DFIS}}=343 \mathrm{~nm} ; \varepsilon_{343}{ }^{\mathrm{DFIS}}=6077 \mathrm{M}^{-1} \mathrm{~cm}^{-1}\right)$, por el contrario, la $\lambda_{\max }$ de absorción del 7,7a-DHBF varía considerablemente según el solvente en el que se encuentra disuelto $\left(\lambda_{\max }{ }^{\mathrm{DHBF}}=494 \mathrm{~nm} ; \varepsilon_{494} \mathrm{DHBF}^{\mathrm{H}}=8200 \mathrm{M}^{-1} \mathrm{~cm}^{-1}\right.$ en tolueno).

En el ultravioleta, la actinometría se realiza preparando una solución de DFIS, irradiando a distintos tiempos y siguiendo la absorbancia del 7,7a-DHBF a $494 \mathrm{~nm}\left(\lambda_{\max }\right)$ en función del tiempo. En cambio, a $\lambda$ mayores (región del espectro correspondiente al visible), el isómero que se irradia es el 7,7a-DHBF. La intensidad de radiación absorbida por el actinómetro $\left(\mathrm{q}_{\mathrm{p}, \lambda}^{\mathrm{v}}\right)$ puede calcularse a partir de la siguiente ecuación: 


$$
\mathbf{q}_{\mathrm{p}, \lambda}^{\mathrm{v}}=\frac{\mathrm{d}[7,7 \mathrm{a}-\mathrm{DHBF}]}{\mathrm{dt}} \frac{1}{\Phi_{\mathrm{Ac}}}
$$

donde $\Phi_{\mathrm{Ac}}$ es el rendimiento cuántico del actinómetro, cuyo valor es 0,2 para la conversión de DFIS a 7,7a-DHBF por radiación UV y es independiente del solvente usado.

Según la Ley de Lambert-Beer:

$$
[7,7 \mathrm{a}-\mathrm{DHBF}]=\mathrm{A}^{494} / \varepsilon 494 \mathrm{l}
$$

Por lo tanto, la ecuación (8) puede expresarse de la siguiente manera:

$$
q_{p, \lambda}^{v}=\frac{d_{A}^{494} / d t}{\varepsilon_{494} l \Phi_{A c}}
$$

donde $\varepsilon_{494}{ }^{\mathrm{DHBF}}=8200 \mathrm{M}^{-1} \mathrm{~cm}^{-1}$ (en tolueno) y l es el camino óptico de la celda en la cual se mide la absorbancia $(\mathrm{l}=1 \mathrm{~cm})$.

Para determinar el q $\mathrm{q}_{\mathrm{p}, \lambda}$, en el intervalo de 310 - $370 \mathrm{~nm}$, se irradió el actinómetro en las mismas celdas utilizadas en los experimentos y a distintos intervalos de tiempo se midió la absorbancia a $494 \mathrm{~nm}\left(\mathrm{~A}^{494}\right)$. Luego se graficó $\mathrm{A}^{494}$ vs. t, se realizó la regresión lineal y se obtuvo la pendiente de la recta. Utilizando la ecuación (10) se calculó qvp la Figura 6.2 se muestran, a modo de ejemplo, los espectros de absorción obtenidos al irradiar el DFIS a $350 \mathrm{~nm}$ en una actinometría típica.

Cuando más del 99,9\% de la luz es absorbida por el actinómetro, se puede suponer que $\mathrm{q}_{\mathrm{p}, \lambda}^{\mathrm{v}}$ es aproximadamente igual a $\mathrm{q}_{\mathrm{p}, \lambda}^{0}$. De lo contrario, debe realizarse el cálculo empleando la ecuación (4). 

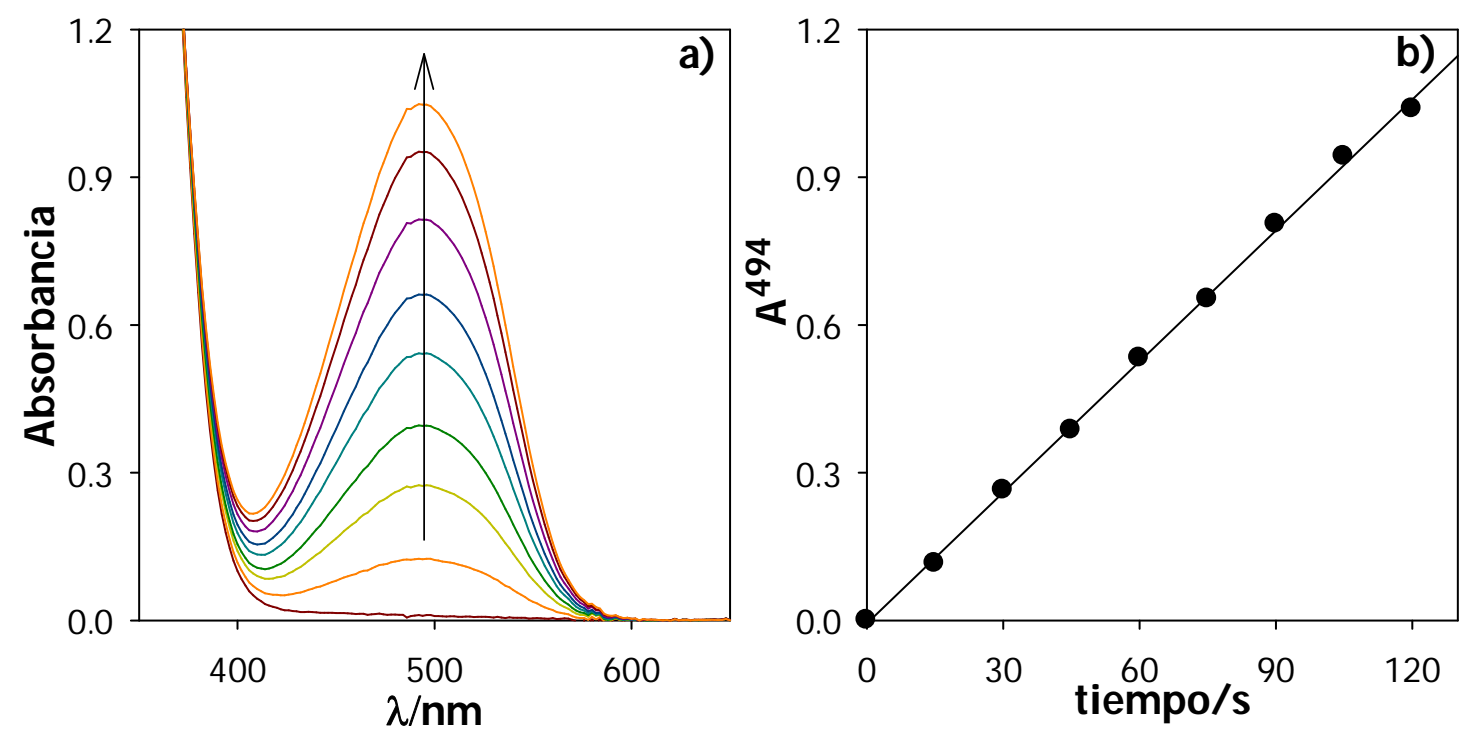

Figura 6.2 a) Evolución de los espectros de absorción de una solución de Aberchrome 540 irradiada a $350 \mathrm{~nm}$. Intervalo de irradiación comprendido entre 0 y 90 s (cada 15 s); l = 1 cm. b) Variación de $\mathbf{A}^{494}$ en función del tiempo de irradiación.

\subsection{Referencias}

1. M. L. Dántola, M. Vignoni, A. L. Capparelli, C. Lorente and A. H. Thomas, Stability of 7,8-dihydropterins in air-equilibrated aqueous solutions, Helvetica Chimica Acta, 2008, 91, 411-425.

2. C. Lorente, A. L. Capparelli, A. H. Thomas, A. M. Braun and E. Oliveros, Quenching of the fluorescence of pterin derivatives by anions, Photochemical and Photobiological Sciences, 2004, 3, 167-173.

3. A. H. Thomas, G. Suárez, F. M. Cabrerizo and A. L. Capparelli, Photochemistry of 6-formylpterin in alkaline medium, Helvetica Chimica Acta, 2001, 84, 3849-3860.

4. P. Salomaa, L. L. Schaleger and F. A. Long, Solvent deuterium isotope effects on acid-base equilibria, Journal of the American Chemical Society, 1964, 86, 1-7.

5. A. M. Braun, M. T. Maurette and E. Oliveros, Photochemical Technology, John Wiley \& Sons, Chichester, 1991. 


\section{Capítulo 5}

\section{Técnicas analíticas}

En el presente trabajo de tesis se utilizaron distintas técnicas de análisis: espectrofotometría UV-visible, espectrofluorometría, cromatografía líquida de alto rendimiento (HPLC), espectrometría de masa, resonancia magnética nuclear (RMN), resonancia paramagnética electrónica $(\mathrm{RPE})$, determinación colorimétrica de peróxido de hidrógeno $\left(\mathrm{H}_{2} \mathrm{O}_{2}\right)$, medida electroquímica del $\mathrm{O}_{2}$ disuelto, investigación de anión superóxido $\left(\mathrm{O}_{2}{ }^{\circ}\right)$. Estas técnicas se utilizaron para obtener información sobre los mecanismos de reacción involucrados, como así también en la identificación y caracterización de productos de reacción y en la cuantificación de reactivos y productos.

\subsection{Espectrofotometría UV-visible}

Esta técnica analítica está basada en la medida directa de la absorción de radiación electromagnética por parte de una muestra, cuantificable a través de la absorbancia y la correlación de esta variable con la concentración de la especie de interés en dicha muestra. Un espectro de absorción UV-visible no proporciona una clara identificación de un compuesto, pero es muy útil para observar cambios en los grupos funcionales de una molécula, debido a que es muy sensible a ellos. Por ejemplo, las distintas formas ácido-base de una misma sustancia pueden claramente ser distinguidas por las diferencias en sus espectros de absorción.

En este trabajo de tesis, los espectros de absorción UV-visible se obtuvieron con los siguientes espectrofotómetros: UV-1800 (Shimadzu), Cary 3 (Varian), HP 8453 y HP 8452A (Hewlett Packard). Estos equipos permiten obtener espectros en el intervalo de longitudes de onda comprendido entre 190 y $900 \mathrm{~nm}$. Los dos primeros equipos mencionados son espectrofotómetros de barrido de doble haz, con alta sensibilidad; mientras que los equipos HP tienen detección por arreglo de diodos de simple haz. Estos equipos son menos sensibles y precisos que el primero, pero tienen la ventaja de ser muy rápidos. Todos poseen programas adecuados para registrar y almacenar los espectros. Estos programas también promedian señales que permiten suavizar los espectros cuando la relación señal/ruido es desfavorable. Los espectros se realizaron utilizando $\mathrm{H}_{2} \mathrm{O}$ como blanco. Para las medidas, se emplearon celdas de cuarzo (Hellma) 
de 0,4 ó 1,0 cm de camino óptico, que se eligieron según la absorbancia de la muestra.

Durante los experimentos se registraron los espectros de absorción, cuyo análisis directo permite decidir si existen cambios espectrales o no. Si hay cambios, se puede afirmar que, según sea el experimento realizado, efectivamente tiene lugar una reacción química o fotoquímica. Si no se observan modificaciones en el espectro, es muy probable que el reactivo sea estable debido a que las pterinas poseen espectros de absorción muy sensibles a los cambios químicos en sus estructuras moleculares.

Con los datos obtenidos por esta técnica se realizaron distintos tipos de gráficos y análisis:

- Absorbancia vs. tiempo, para una dada longitud de onda $(\lambda)$, que permite evaluar aspectos cinéticos del proceso.

- Espectros diferencia experimentales (EDE), donde a cada espectro absoluto obtenido para cada tiempo de reacción $(E(t=t n))$ se le resta el espectro absoluto obtenido a tiempo "cero" $(E(t=0))$. Por ejemplo: $E D E(t=1)=E(t=1)-E(t=0)$. Esta operación implica restar para cada longitud de onda la absorbancia de $E(t=0)$ a la absorbancia de $E(t=11)$; por lo tanto, para cada reacción se obtiene un espectro por cada tiempo distinto de cero en el que fue analizada la solución ( $\operatorname{EDE}(\mathrm{t}=\mathrm{tn}), \operatorname{con} \mathrm{n}=1,2,3, \ldots)$. En este análisis se tuvo en cuenta la presencia y constancia en el tiempo de puntos isosbésticos, ya que si éstos se mantienen, se puede inferir que un único proceso químico tiene lugar. Además, son muy útiles para evaluar el momento en el cual las reacciones secundarias se tornan importantes. Así, se determina el tiempo máximo de reacción dentro del cual se harán otros tipos de análisis espectrofotométricos o se aplicarán otras técnicas en los cuales se desea aislar el primer proceso.

- Espectros diferencia experimentales normalizados (EDEN), que se obtuvieron dividiendo la absorbancia a cada $\lambda$ por un número elegido arbitrariamente según la conveniencia de cada caso, generalmente donde se observan cambios espectrales importantes. Si en una reacción existe un único proceso, los EDEN a distintos tiempos serán iguales dentro del error experimental, mientras que serán diferentes si hay más de un proceso. Este criterio también se utilizó para determinar durante cuánto tiempo, podía considerarse la existencia de un único proceso (primario).

- Espectros diferencia teóricos (EDT) y los respectivos espectros normalizados (EDTN). Se utilizaron principalmente en la identificación de productos. Para esto, primero se propone una o más sustancias como posibles productos de una reacción, basándose en literatura o trabajos previos. Luego se registran los espectros del reactivo 
y de los supuestos productos. Si la estequiometria propuesta es 1:1 (caso más común), las soluciones preparadas deberían ser de igual concentración, de lo contrario deben corregirse los espectros. Posteriormente, se resta el espectro de los productos al espectro del reactivo obteniéndose el correspondiente EDT. Finalmente, dividiendo EDT por un determinado valor se obtiene el EDTN.

\subsection{Espectrofluorometría}

Según lo expuesto en el Capítulo 3, las pterinas aromáticas emiten radiación cuando son excitadas a ciertas longitudes de onda. En este caso, se registraron espectros de emisión en un rango dado de longitudes de onda $\left(\lambda_{\text {em }}\right)$ excitando a una longitud de onda fija $\left(\lambda_{\text {exc }}\right)$. El máximo de emisión se encuentra cercano a $450 \mathrm{~nm}$, aunque se observa un pequeño corrimiento batocrómico de la banda de emisión de las formas alcalinas respecto de las formas ácidas. Estos compuestos aunque generan estados tripletes, poseen emisión de fosforescencia despreciable en $\mathrm{H}_{2} \mathrm{O}$ a temperatura ambiente..$^{1,2}$

En este trabajo, se realizaron medidas de emisión de fluorescencia en estado estacionario empleando un espectrofluorómetro Perkin-Elmer LS50B. El equipo cuenta con una lámpara de Xenón, un monocromador de excitación que focaliza el haz sobre la cubeta, un monocromador de emisión y como detector, un fotomultiplicador R928. Además, el equipo utiliza el programa de adquisición de datos FL WinLab v. 2.7.0. El espectro de emisión obtenido debe corregirse por lo que se denomina respuesta del instrumento, dado que la sensibilidad del detector y la eficiencia de los monocromadores varían con la longitud de onda. Esta corrección se realiza dividiendo la señal de emisión de la muestra por la respuesta del instrumento. En todos los experimentos realizados con este equipo, se utilizaron celdas para fluorescencia de cuarzo de $1 \mathrm{~cm}$ x $1 \mathrm{~cm}$ y de $1 \mathrm{~cm} \mathrm{x} \mathrm{0,4} \mathrm{cm.} \mathrm{Se} \mathrm{utilizaron} \mathrm{soluciones} \mathrm{acuosas} \mathrm{a} \mathrm{temperatura}$ ambiente. La longitud de onda de excitación utilizada fue la del máximo de absorción de la banda de menor energía de cada compuesto, por lo cual fue variando entre 330 y 365 $\mathrm{nm}$.

\subsection{Cromatografía Líquida de Alto Rendimiento}

High performance liquid chromatography (HPLC), es una técnica que se utiliza para separar componentes de una mezcla basándose en distintos tipos de interacciones 
químicas o físicas generadas entre las sustancias analizadas y la columna cromatográfica. También puede utilizarse como criterio de pureza, para cuantificar e identificar compuestos.

En esta técnica, el compuesto atraviesa la columna cromatográfica (fase estacionaria) mediante el bombeo del solvente (fase móvil). Como la fase estacionaria está compuesta por partículas pequeñas densamente empaquetadas, ofrece gran resistencia al flujo de la fase móvil, por lo cual se requiere una alta presión para que el solvente fluya a una velocidad adecuada. El tiempo que tarda un compuesto en ser eluido de la columna se llama tiempo de retención $\left(t_{r}\right)$, el cual depende de la naturaleza misma del compuesto, de la composición de la fase estacionaria y de la fase móvil. Se denomina cromatograma a la gráfica de la señal en función del tiempo de corrida. Esta señal es un valor dado en unidades arbitrarias (u. a.). En el caso de utilizar detector con arreglo de diodo UV/Vis, la señal es proporcional a la absorbancia de la solución a la longitud de onda seleccionada.

En este trabajo se utilizó esta técnica con dos objetivos fundamentales: para obtener los perfiles de concentración de consumo de reactivo y formación de productos que tienen lugar a lo largo de una reacción, y para identificar productos de reacción. Para esto se utilizaron:

* EQUIPOS HPLC: Para estas medidas se emplearon dos equipos diferentes:

Equipo HPLC I: Cromatógrafo Shimadzu Prominence LC-20A. Este equipo cuenta con un sistema de detección formado por un detector con arreglo de diodo UV/ VIS (SPD-M20A, Shimadzu), el cual permite hacer un monitoreo a todas las $\lambda$ entre 200 y $800 \mathrm{~nm}$. El equipo posee un programa de adquisición de datos (LC Solution) que permite registrar y analizar los espectros de absorción de cada uno de los productos (Figura 5.1).

Equipo HPLC II: Cromatógrafo Waters 2695 Separation Module (Alliance) con un sistema de detección de arreglo de diodos (Waters 996 PDA Detector) y un programa de adquisición de datos Empower 3 (Chromatography Data System, CDS).

* COLUMNAS: En general se utilizaron columnas de fase reversa C-18 de diferentes marcas comerciales: Pinnacle-II C18 (250 mm x 4,6 mm, $5 \mu \mathrm{m}$; Restek), LiChro CART RP 18 (250 mm x 4 mm, $5 \mu \mathrm{m}$, Merck), XTerra RP18 (250 mm x 4,6 mm, $5 \mu \mathrm{m}$, Waters) y Synergi Polar-RP (150 mm $\times 4,6 \mathrm{~mm}, 4 \mu \mathrm{m}$; Phenomenex). Esta última, posee un fenilo 
unido con función éter a la fase fija con protección hidrofílica diseñada para maximizar la retención y la selectividad de los analitos polares y aromáticos.

* SOLVENTES: Se utilizaron distintas mezclas según los compuestos a analizar. A continuación se expresa la composición de dichas mezclas como relación de volúmenes y el pH corresponde a la solución acuosa antes mezclarse con el solvente orgánico:

- 95 - 96 \% 15 mM TRIS-HCl - 1 mM NaCl y 4 - 5 \% metanol, $\mathrm{pH}=6,8$ a flujo 1 $\mathrm{ml} / \mathrm{min}$

- 96 - $100 \% 10 \mathrm{mM} \mathrm{NH}_{4} \mathrm{Ac}$ y 0 - $4 \%$ metanol, $\mathrm{pH}=7,0$ a flujo $0,6 \mathrm{ml} / \mathrm{min}$

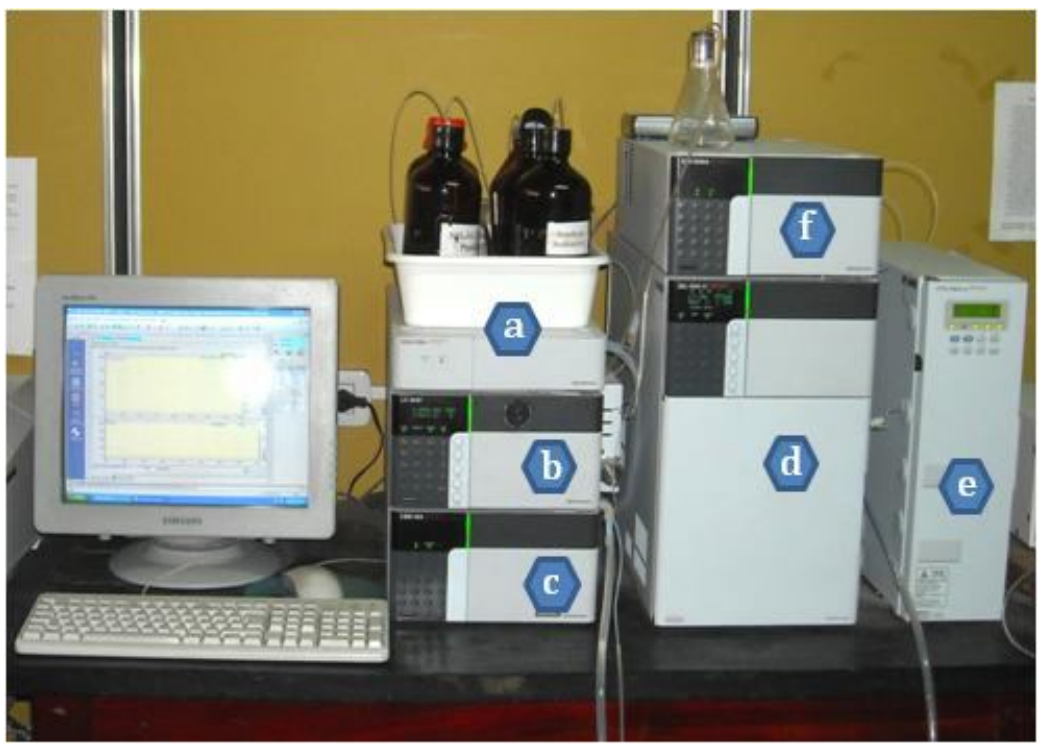

Figura 5.1 Fotografía del Equipo HPLC I. a) Solventes y desgasificador, b) bomba, c) controlador, d) autosampler, e) horno y columna, f) detector UV-Vis.

Para la identificación de los productos se realizaron corridas cromatográficas con soluciones de patrones comerciales. Se compararon los valores de $t_{R}$ y los espectros de absorción con los de los productos obtenidos en los distintos experimentos.

Para la cuantificación de las sustancias separadas por esta técnica, se realizaron curvas de calibración empleando soluciones patrón. Se prepararon las soluciones de mayor concentración por pesada y luego se prosiguió de dos maneras diferentes: (i) se prepararon soluciones de menor concentración por dilución con $\mathrm{H}_{2} \mathrm{O}$ y se inyectó siempre el mismo volumen en el HPLC, o (ii) se realizaron inyecciones de distintos volúmenes de la misma solución inicial concentrada. A partir de los cromatogramas se integró el área de los picos y se construyeron curvas de área en función de la concentración. A estas se le aplicó una regresión lineal de la cual se obtuvo una pendiente, cuyo valor sería posteriormente utilizado como factor de conversión En la 
Figura 5.2 se muestran a modo de ejemplo un conjunto de corridas cromatográficas y la correspondiente curva obtenida. Estas curvas de calibración se realizaron para todas las sustancias que se necesitaba cuantificar y en las mismas condiciones de corrida en las cuales se realizaron luego los distintos experimentos.
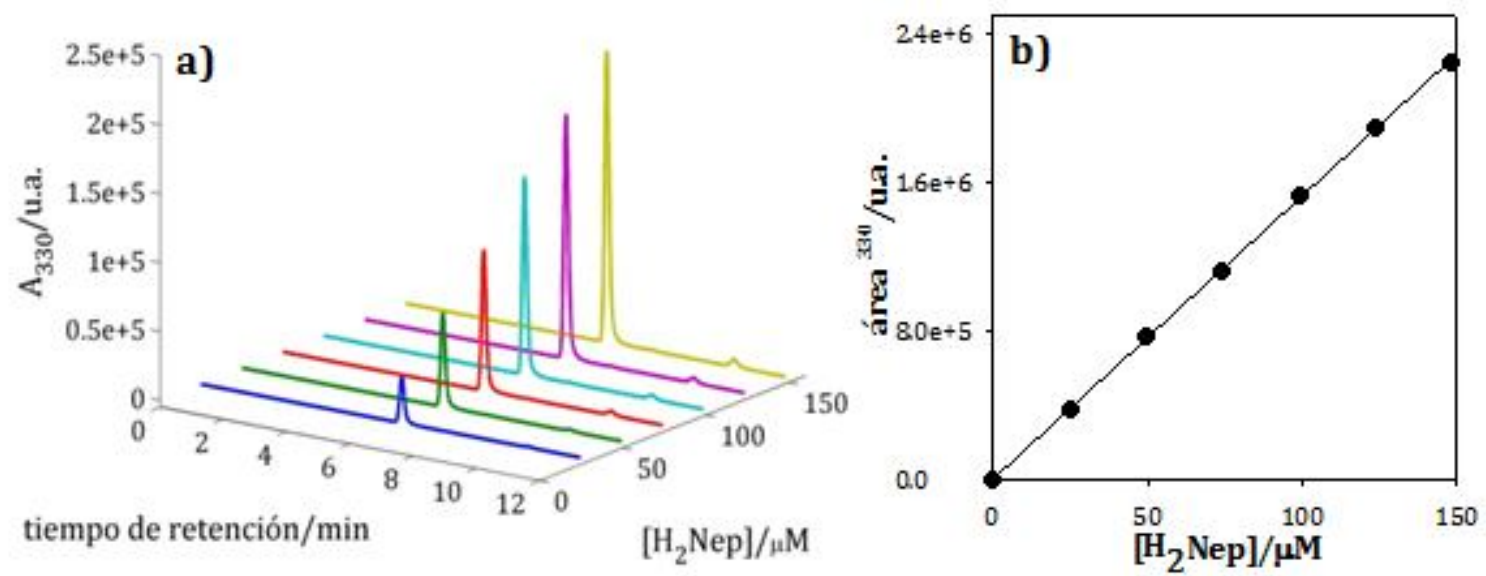

Figura 5.2 Ejemplo de curva de calibración: a) cromatogramas de distintas concentraciones de $\mathrm{H}_{2} \mathrm{Nep}$, realizadas con el Equipo HPLC I, columna Synergi Polar-RP, solvente de corrida (a); b) Gráfico área vs. [ $\left.\mathrm{H}_{2} \mathrm{Nep}\right], \lambda_{\mathrm{an}}=330 \mathrm{~nm}$.

Debido a su gran similitud estructural, muchos de los 7,8-dihidroderivados poseen tiempos de retención similares a los de sus análogos oxidados, lo cual genera una gran dificultad para lograr una buena separación. En algunos casos fue necesario utilizar un método matemático para obtener los perfiles de concentración de reactivos y productos. Primero, se obtienen las curvas de calibración a dos $\lambda$ distintas en las cuales se observen diferencias significativas en los espectros de absorción de ambos compuestos. Si se considera que un pico del cromatograma corresponde sólo a dos compuestos, y si se asume aditividad de las áreas, la concentración de ambos puede ser calculada usando un sistema de dos ecuaciones con dos incógnitas, como se muestra a continuación:

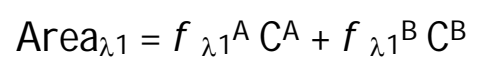

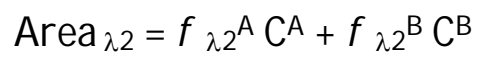

donde Area $_{\lambda 1}$ y Area $\lambda_{22}$ son los valores de las áreas que resultan de la integración de los cromatogramas a las longitudes de onda $\lambda 1$ y $\lambda 2, \mathrm{C}^{\mathrm{A}}$ y $\mathrm{C}^{\mathrm{B}}$ son las concentraciones de $\mathrm{A}$ y de $\mathrm{B}, \mathrm{y}_{\lambda 1^{\mathrm{A}}}, \mathrm{f}_{\lambda 1^{\mathrm{B}}}, \mathrm{f}_{\lambda 2^{\mathrm{A}}}$ y $\mathrm{f}_{\lambda 2^{\mathrm{B}}}$ son los factores obtenidos de las curvas de calibración de $\mathrm{A} y$ $\mathrm{B}$ a $\lambda 1$ y $\lambda 2$. Varias ecuaciones fueron utilizadas para controlar los resultados obtenidos. Para esto se trabajo a diferentes $\lambda$, encontrando que los resultados obtenidos son 
consistentes independientemente del par de $\lambda$ seleccionadas.

\subsection{Espectrometría de Masa}

Esta técnica se utiliza para determinar con gran precisión la masa de ciertos compuestos y por consiguiente, ayudar a su identificación. El espectrómetro de masas genera la ionización de moléculas que luego, mediante un campo eléctrico y/o magnético, se separan en función de su relación masa/ carga $(\mathrm{m} / \mathrm{z})$. Cuando este haz de iones formado alcanza el detector produce un patrón específico, que permite caracterizar el compuesto bajo análisis.

La cámara o fuente de ionización es el elemento que convierte los componentes de una muestra (analito) en iones, obteniéndose así un haz de iones positivos o negativos. Existen distintos tipos de fuentes. En este caso, los dos equipos utilizados poseen ionización por electrospray (ESI), donde a partir de una solución de analito, se genera una nube formada por muy pequeñas gotas cargadas eléctricamente. Estas gotas, al ser sometidas a un campo eléctrico, disminuyen su tamaño y pasan a la fase gaseosa cuando las fuerzas coulómbicas de repulsión entre los iones con múltiple carga generados en su interior vencen la tensión superficial (evaporación iónica).

Luego, los iones pasan al analizador de masas, donde su trayectoria o velocidad son afectadas mediante un campo eléctrico o magnético (Figura 5.3). El dispositivo más utilizado para tal fin es el cuadrupolo, que posee un conjunto de cuatro barras cilíndricas de metal que sirven de electrodos del filtro de masas. Los iones de la fuente son acelerados por un potencial de 5 a $15 \mathrm{~V}$ e introducidos en el espacio entre las barras. Cada par de barras opuestas se conectan a un polo positivo y a un polo negativo y se aplica un potencial de voltaje variable de corriente alterna. Esto hace que los iones dentro de un intervalo limitado de valores de $\mathrm{m} / \mathrm{z}$, circulen con una trayectoria rectilínea y lleguen al detector. El centro de esta banda puede variarse ajustando los potenciales de corriente. Otro analizador utilizado es el TOF (time of flight), donde la separación se genera fundamentalmente por las distintas velocidades de vuelo de los iones y el tiempo que tardan en llegar al detector.

Finalmente, el detector registra la carga inducida o la corriente producida cuando un ión pasa cerca o golpea una superficie. Generalmente, se utiliza un cierto tipo de multiplicador de electrones (electromultiplicador), cuyo funcionamiento se basa en el efecto cascada producido al impactar un determinado ion (o iones) en el mismo. 


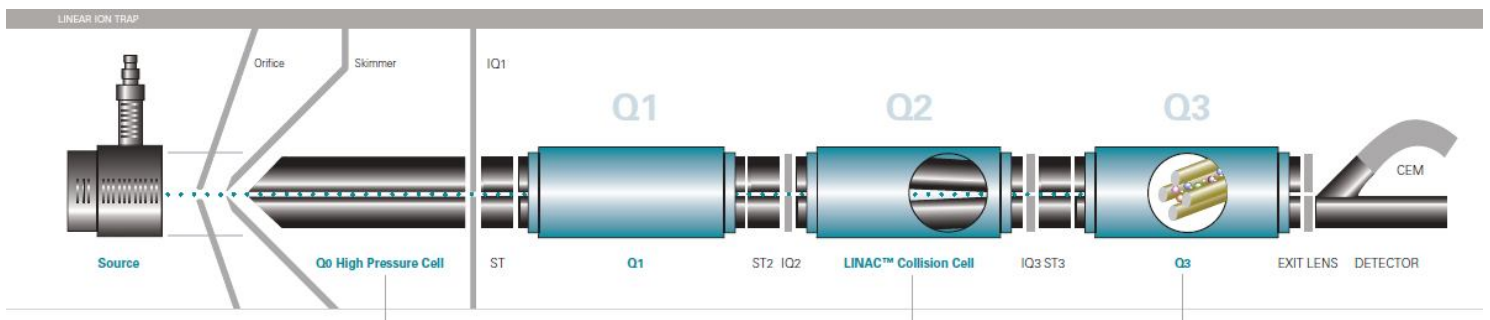

Figura 5.3 Esquema de un espectrómetro de masa Q TRAP Applied Biosystems (tomado de AB Sciex).

En este trabajo se utilizaron dos espectrómetros de masa, ambos acoplados a HPLC:

Equipo MS I: Q TRAP Applied Biosystems (AB Sciex Instruments 027130) acoplado a un HPLC Agilent 1100 con columna XTerra RP18, previamente descripta y solvente de corrida (b). La fuente de ionización es por electrospray ESI (Turbo Ion Spray (TIS)) operada en modo positivo y negativo. Se utilizó nitrógeno como gas nebulizador y la temperatura del capilar de espray fue de $450^{\circ} \mathrm{C}$. El potencial declaustering (DP) fue de 60 V. El rango de masas analizado fue de 150 a 500 umas con una resolución de 0,1 uma. Cuando fue posible, se realizó la identificación de compuestos comparando con los espectros de masa y tiempos de retención de patrones. Previamente a la inyección de la muestra, ésta se filtró empleando filtros de nylon $(0,22 \mu \mathrm{m})$. Se utilizó el programa de adquisición de datos de análisis Analyst 1.4.2. (Figura 5.4)

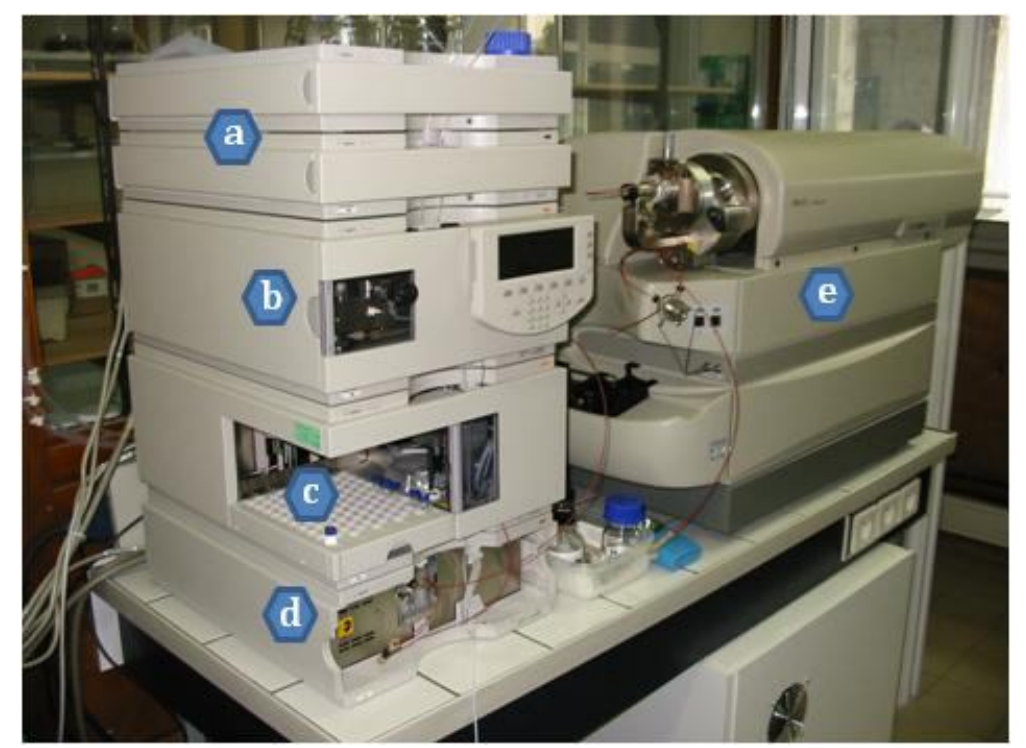

Figura 5.4 Fotografía del Equipo MS I. a) Solventes y desgasificador, b) bomba, c) autosampler, d) horno y columna, e) espectrómetro de masa.

Equipo MS II: Q TOF Ultima API (Waters) acoplado al Equipo HPLC-2 (Waters) con la columna XTerra RP18 y solvente de corrida (b). Posee un electrospray ESI, utilizado en modo positivo (ESI+). Los voltajes del capilar, cono y lentes $\mathrm{RF}$ fueron de 3,5 kV, $35 \mathrm{~V} \mathrm{y}$ $40 \mathrm{~V}$, respectivamente. La temperatura de la fuente fue de $120^{\circ} \mathrm{C}$ y la de desolvatación 
fue de $150{ }^{\circ} \mathrm{C}$. La energía del gas de colisión (Ar) se fijó en 10 eV en el modo TOF MS. Se utilizó $\mathrm{N}_{2}$ como gas nebulizador. Se adquirieron los datos con el programa Masslynx 4.1 (Waters).

\subsection{Resonancia Magnética Nuclear}

La espectroscopia de resonancia magnética nuclear (RMN) se utiliza principalmente para identificar y analizar compuestos orgánicos. Esta técnica sólo sirve para estudiar núcleos atómicos con un número impar de protones o neutrones (o de ambos), como por ejemplo de ${ }^{1} \mathrm{H},{ }^{13} \mathrm{C},{ }^{19} \mathrm{~F}$ y ${ }^{31} \mathrm{P}$. Estos núcleos poseen un espín y son activos magnéticamente, por lo cual poseen un movimiento de rotación sobre su eje, comportándose como pequeños imanes. En ausencia de campo magnético estos espines se orientan al azar, pero al aplicar un campo, los núcleos con espines positivos se orientan en dirección del campo (con mínima energía, espín $\alpha$ ), mientras que los núcleos con espín negativo se orientan en dirección opuesta a la del campo magnético (en estado de mayor energía, espín $\beta$ ). La diferencia $\Delta E$ entre estos dos estados de energía depende de la fuerza del campo magnético aplicado $\left(\mathrm{H}_{0}\right)$ : a mayor campo, mayor diferencia de energía entre los dos estados.

Cuando una muestra es irradiada con radiación electromagnética de radiofrecuencia apropiada, es decir la energía aplicada es exactamente igual a la energía existente entre los dos estados de energía, los núcleos absorben la radiación y pasan del estado de menor al de mayor energía. Así, los núcleos están en resonancia con el campo aplicado. Luego, debe restablecerse la condición de equilibrio entre los niveles de energía, es decir debe producirse la relajación. Este fenómeno es detectado por el equipo (Figura 5.5) y las señales son registradas en un gráfico de frecuencias vs. intensidad, llamado transformada de Fourier (FT-RMN).

Los núcleos se encuentran rodeados de electrones que se mueven generando un campo magnético inducido que se opone al campo magnético externo. De esta manera, el campo magnético que realmente llega al núcleo es más débil que el campo externo aplicado y, por lo tanto, se dice que el núcleo está protegido o apantallado. Esto hace que los protones con entornos electrónicos diferentes, se encuentran desigualmente protegidos o apantallados, resuenen a distintas frecuencias, y por lo tanto, tengan distintos desplazamientos químicos (con unidades de $\delta$ ó partes por millón (ppm)). Como resultado se obtiene un espectro de diversas frecuencias donde cada conjunto de núcleos específicos genera una señal de RMN, según el distinto apantallamiento de los 
núcleos.

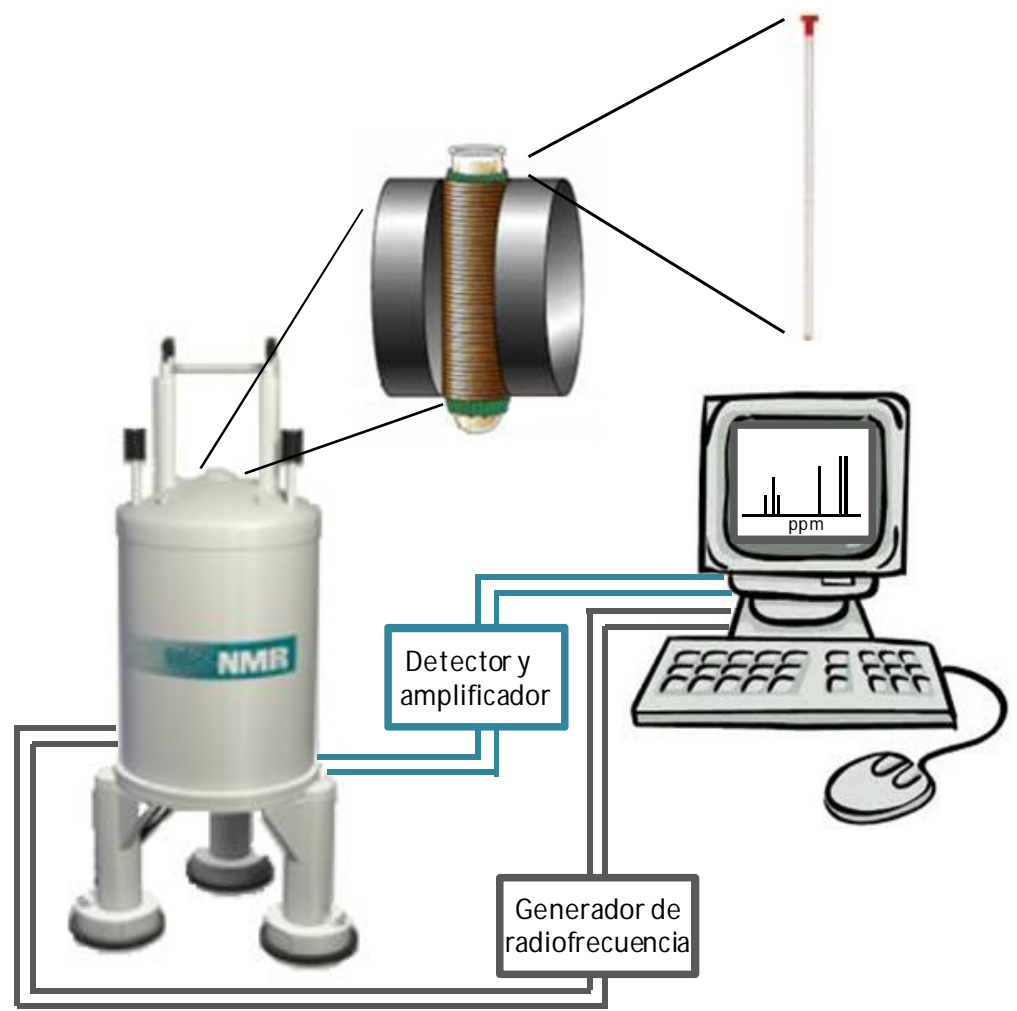

Figura 5.5 Esquema de un equipo de resonancia magnética nuclear.

Es difícil medir con exactitud el campo magnético al que un protón absorbe y distinguir protones individuales, ya que las absorciones varían muy poco. Por lo tanto, se utiliza un compuesto de referencia y se determina la diferencia de intensidades de campo magnético necesario para la resonancia de los protones de la muestra y de la referencia. Para esto se utiliza tetrametilsilano (TMS, $\left.\left(\mathrm{CH}_{3}\right)_{4} \mathrm{Si}\right)$, donde al ser el silicio menos electronegativo que el carbono, los metilos del TMS están fuertemente apantallados. De esta manera, estos protones resuenan a mayores campos que el resto de los protones unidos a carbono u otro elemento y así las señales de RMN aparecen a campos más bajos. Generalmente se utiliza la escala $\delta$ (delta) para el desplazamiento químico, en la que la absorción del TMS se define como $0.00 \delta$. La mayoría de los protones absorben a menores campos y la escala $\delta$ aumenta hacia campos menores. Las señales de Hvarían entre 0 y $12 \delta$.

En este trabajo se realizaron sólo experimentos de RMN protónico ( $\left.{ }^{1} \mathrm{H}-\mathrm{RMN}\right)$. Para esto se utilizó agua deuterada $\left(\mathrm{D}_{2} \mathrm{O}\right)$ como solvente. Es necesario aclarar, que el $\mathrm{D}_{2} \mathrm{O}$ siempre posee una pequeña cantidad de $\mathrm{H}_{2} \mathrm{O}$, la cual puede incrementarse cuando la solución está en contacto con la humedad del ambiente. Se disolvió el compuesto a estudiar (Sección 4.2.3) y se ajustó el pH de la misma según lo deseado (es decir, $\approx 7,0$ 
para compuestos dihidroderivados $\mathrm{y} \approx 10,5$ para las pterinas oxidadas). Luego se colocaron 0,7 $\mathrm{ml}$ de la solución en un tubo de $\mathrm{RMN}$ de $5 \mathrm{~mm}$ de diámetro y se tomaron los respectivos espectros de ${ }^{1} \mathrm{H}-\mathrm{RMN}$. Cabe destacar que al utilizar $\mathrm{D}_{2} \mathrm{O}$ como solvente, solo se detectan los protones no intercambiables con D (deuterio), es decir los unidos a $\mathrm{C}$ (no así, los unidos a $\mathrm{N} \mathrm{u} \mathrm{O).} \mathrm{Para} \mathrm{estudiar} \mathrm{la} \mathrm{fotoquímica} \mathrm{de} \mathrm{los} \mathrm{compuestos,} \mathrm{algunos}$ tubos fueron irradiados con luz UV-A. Cuando fue necesario, se eliminó el $\mathrm{O}_{2}$ de la solución burbujeando los tubos con $\mathrm{N}_{2}$ (Sección 4.2.4).

Se utilizó un espectrómetro Bruker AM-500. El número de acumulaciones varió entre 8 y 64 en función de la concentración de la muestra. Los experimentos se realizaron a un campo magnético de $11.743 \mathrm{~T}$, es decir, a una frecuencia de $500 \mathrm{MHz}$ para el protón, y a una temperatura de $300 \mathrm{~K}$. Se utilizó el pico correspondiente al $\mathrm{H}_{2} \mathrm{O}$ para calibrar los espectros $(\delta=4,79)$.

\subsection{Resonancia Paramagnética Electrónica}

La técnica de resonancia paramagnética electrónica (RPE) se utiliza generalmente para investigar sistemas moleculares que involucren fragmentos con electrones desapareados. La técnica se basa en los mismos principios que la resonancia magnética nuclear, analizando la interacción entre radiación electromagnética y momentos magnéticos, pero en el caso de RPE, el momento magnético proviene del electrón y no del núcleo (Figura 5.6).

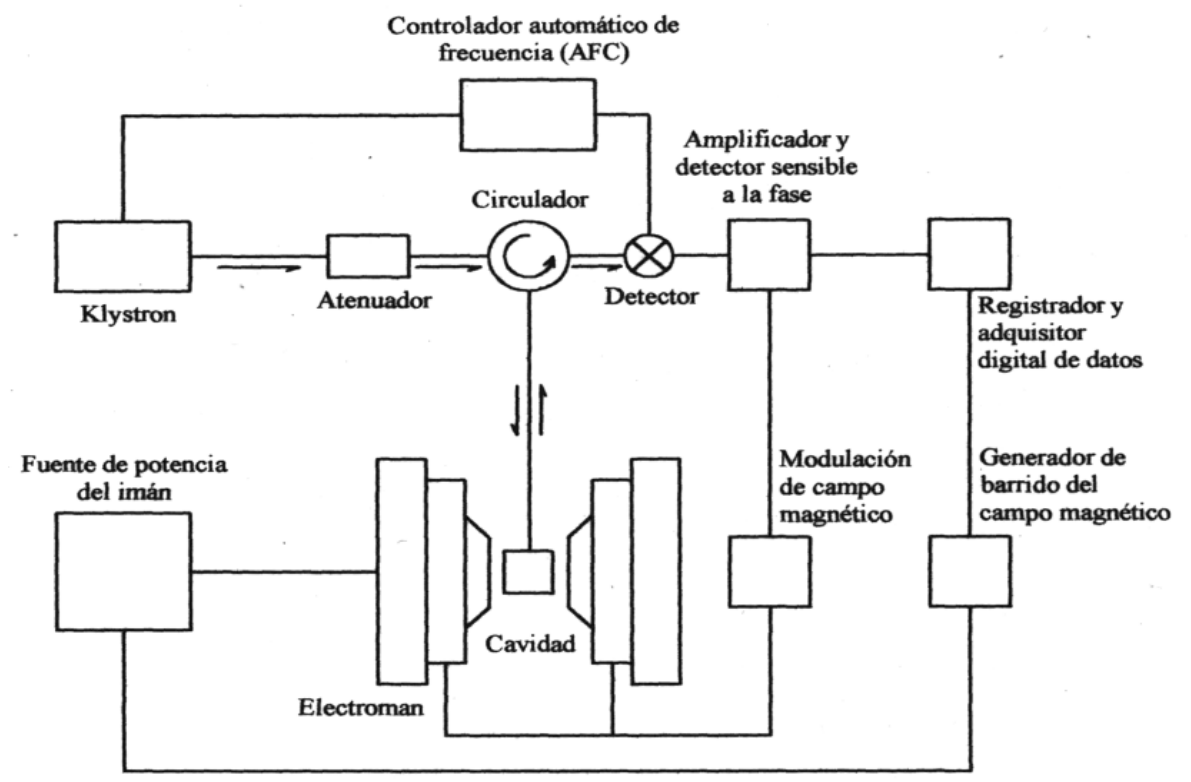

Figura 5.6 Esquema de un espectrómetro básico de RPE ${ }^{3,4}$

Se utilizó un espectrómetro Elexys E500 BRUKER. Los parámetros se ajustaron 
utilizando soluciones estándar. La potencia de microondas fue de $20 \mathrm{~mW}$; la amplitud de modulación de campo de 0,1 mT; la frecuencia de modulación de campo de $100 \mathrm{kHz}$ y la frecuencia de microondas de 9,77 GHz. Las irradiaciones se realizaron dentro de la cavidad resonante del espectrómetro utilizando una lámpara de mercurio Oriel (Palaiseau, France). La luz fue remitida mediante fibra óptica, directo a la cavidad. En este caso la irradiación fue de luz policromática.

En este trabajo de tesis, la técnica se utilizó con el objetivo de investigar la generación de $\mathrm{O}_{2}{ }^{-}$. Para esto, se utilizó la nitrona 5,5-dimetil-1-pirrolin-N-óxido $(\mathrm{DMPO})^{5}$ que actúa como secuestrador (spin trapping). Es decir, DMPO se une a radicales transientes formando aductos radicales más estables (de mayor vida media) y que generan señales características, los cuales pueden ser detectados por RPE con mayor facilidad.6,7 (Figura 5.7)

a)<smiles>CC1(C)CCC=[N+]1[O-]</smiles>

DMPO<smiles>[O-]</smiles>

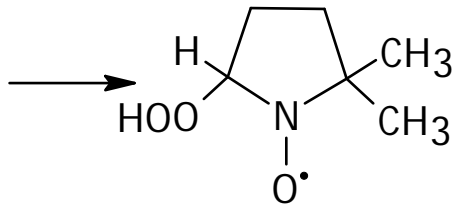

DMPO $-00 H$

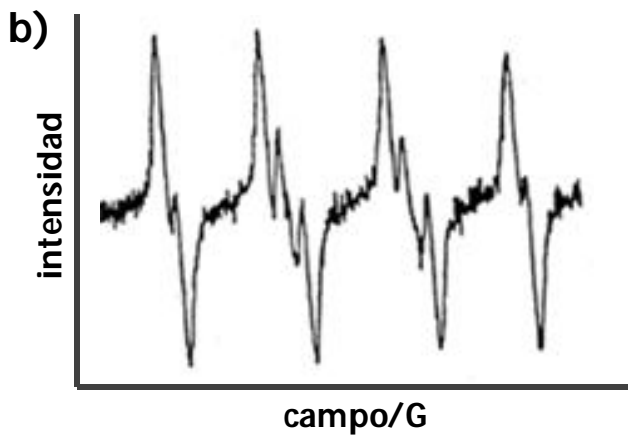

Figura 5.7 a) Reacción química entre el DMPO y $\mathrm{O}_{2}{ }^{-}$. b) Señal de RPE correspondiente al aducto DMPO-OOH.

Las muestras consistieron en soluciones acuosas conteniendo (volumen final de $500 \mu \mathrm{l}) 100 \mu \mathrm{M}$ de pterina oxidada y $50 \mathrm{mM}$ DMPO en buffer Tris-HCl $1 \mathrm{mM}$ y NaCl 0,5 $\mathrm{mM}(\mathrm{pH}=7,2)$. Las muestras fueron transferidas a celdas planas de cuarzo de $0,2 \mathrm{~cm}$ de camino óptico y fueron irradiadas a temperatura ambiente. Los espectros fueron tomados a distintos tiempos desde el comienzo de la irradiación y hasta llegar a un plateau en la intensidad de la señal. Para confirmar la formación de $\mathrm{O}_{2}{ }^{-{ }^{-}}$, se realizaron experimentos similares en presencia de SOD $(22 \mathrm{U} / \mathrm{ml}),{ }^{8}, 9$ la cual elimina el $\mathrm{O}_{2}{ }^{-}$ catalizando su conversión a $\mathrm{H}_{2} \mathrm{O}_{2}$ y $\mathrm{O}_{2}$. Además, se realizaron experimentos control en presencia de SOD desnaturalizada por calor por 30 min a $90{ }^{\circ} \mathrm{C}^{10}$

\subsection{Detección de peróxido de hidrógeno}

Para estudiar la formación de esta especie reactiva, se utilizó un kit comercial "COLESTAT enzimático" provisto por laboratorios Wiener (Argentina). Este kit se 
emplea para determinar colesterol en sangre y se basa en las siguientes reacciones:

$$
\begin{array}{rlrl}
\text { éster de colesterol }+\mathrm{H}_{2} \mathrm{O} & \stackrel{\text { Lipasa }}{\longrightarrow} \text { colesterol }+\mathrm{ROOH} \\
\text { colesterol }+\mathrm{O}_{2} & \stackrel{\mathrm{CHOD}}{\longrightarrow} \text { colest-4-en-3-ona }+\mathrm{H}_{2} \mathrm{O}_{2} \\
\text { 4- } \mathrm{AF}+\text { fenol }+2 \mathrm{H}_{2} \mathrm{O}_{2} & \stackrel{\mathrm{POD}}{\longrightarrow} & 4 \text {-(p-benzoquinona monoimino)-fenazona }+4 \mathrm{H}_{2} \mathrm{O}
\end{array}
$$

donde Lipasa es, en particular lipasa fungal, CHOD representa la enzima colesterol oxidasa, mientras que POD la peroxidasa. Entonces, el colesterol se libera de sus ésteres y luego se oxida para dar una cetona y $\mathrm{H}_{2} \mathrm{O}_{2}$. Finalmente, el $\mathrm{H}_{2} \mathrm{O}_{2}$ se hace reaccionar con 4-aminofenazona y fenol para dar una quinona coloreada que puede cuantificarse por espectrofotometría.

Esta última reacción es la que se emplea para medir $\mathrm{H}_{2} \mathrm{O}_{2}$ en solución. Por ende, se modificó el método de determinación de colesterol para cuantificar $\mathrm{H}_{2} \mathrm{O}_{2}$, agregando el reactivo comercial a la muestra que contiene $\mathrm{H}_{2} \mathrm{O}_{2}$ y la cantidad de quinona coloreada obtenida se la compara con la de una curva de calibración llevada a cabo con solución patrón de $\mathrm{H}_{2} \mathrm{O}_{2}$. Las otras dos enzimas no interfieren dado que son específicas para los sustratos indicados previamente. Igualmente, se realizaron controles para descartar interferencias, por ejemplo aplicando el método a soluciones no irradiadas de pterinas para descartar la existencia de reacciones inespecíficas.

El reactivo de trabajo se prepara colocando en una probeta 50 partes de agua destilada, 5 partes de reactivo 4 -aminofenazona (4-AF) y 5 partes de reactivo fenol y se lleva a 100 partes con agua destilada. Luego se agregan 2 partes de las enzimas previamente homogeneizadas. Se mezcla por inversión, sin agitar. Se pueden preparar distintas cantidades respetando las proporciones establecidas, además de respetar el orden de agregado de los reactivos y asegurar una perfecta homogeneización de los mismos, a fin de evitar que el reactivo fenol deteriore las enzimas, provocando una alteración en la calidad del reactivo de trabajo. Las cantidades y concentraciones de los componentes son las siguientes: 1,25 mM 4-aminofenazona, 2,75 $\mathrm{mM}$ fenol, lipasa $\geq$ $6000 \mathrm{U} / \mathrm{l}, \mathrm{CHOD} \geq 60 \mathrm{U} / \mathrm{l}, \mathrm{POD} \geq 400, \mathrm{pH} 7,4 \pm 0,1$. Este reactivo, puede almacenarse en frasco de vidrio color caramelo y conservar en refrigerador por aproximadamente 1 mes a partir del momento de su preparación. Los reactivos provistos son estables en refrigerador $\left(2-10^{\circ} \mathrm{C}\right)$ hasta la fecha de vencimiento indicada por el fabricante.

Para la mezcla de reacción, se manipularon distintos volúmenes según la cantidad de $\mathrm{H}_{2} \mathrm{O}_{2}$ sospechada en la muestra. Normalmente se utilizaron $0,2 \mathrm{ml}$ de muestra y 0,9 
$\mathrm{ml}$ de reactivo; mientras que para concentraciones bajas de $\mathrm{H}_{2} \mathrm{O}_{2}$ se emplearon $0,5 \mathrm{ml}$ de muestra y 0,6 $\mathrm{ml}$ de reactivo. Luego de adicionar la muestra al reactivo, se deja a temperatura ambiente $\left(25^{\circ} \mathrm{C}\right)$ por 30 minutos y se leen las absorbancias a $505 \mathrm{~nm}\left(\mathrm{~A}^{505}\right)$. Estos valores, como también los obtenidos para las soluciones de $\mathrm{H}_{2} \mathrm{O}_{2}$ preparadas para realizar la curva de calibración, fueron corregidos por el valor de absorbancia a la misma longitud de onda del blanco de reacción, el cual se preparó mezclando el reactivo colorimétrico de trabajo con la solución de pterina antes del inicio de la reacción a estudiar (en idénticas proporciones a las usadas para la determinación de $\mathrm{H}_{2} \mathrm{O}_{2}$ a los distintos tiempos de reacción).

Para crear la curva de calibración, se prepararon soluciones acuosas de $\mathrm{H}_{2} \mathrm{O}_{2}$ de distinta concentración. Primero, se hicieron diluciones de la solución concentrada $\left(\mathrm{H}_{2} \mathrm{O}_{2}\right.$ $30 \%, \sim 10$ M) hasta obtener una solución cuya absorbancia a $254 \mathrm{~nm}$ esté comprendida entre 0,3 - 1, donde el error en la medida de la absorbancia es muy pequeño. Luego, se tomaron los espectros y registraron los valores de absorbancia a $254 \mathrm{~nm}$ con los cuales se calcularon las concentraciones empleando la ecuación de Lambert-Beer $\left(\varepsilon^{254 \mathrm{~nm}}=18,4\right.$ $\mathrm{M}^{-1} \mathrm{~cm}^{-1}$ ). Para preparar soluciones de menor concentración se realizaron distintas diluciones $(1 / 100,1 / 1000$, etc.) de las soluciones anteriores. Estas soluciones se trataron de igual forma que las muestras previamente descriptas. Se realizaron 2 curvas, una para 0,2 ml y otra para 0,6 ml de volumen de muestra. Se midió la $\mathrm{A}^{505}$ (Figura 5.8.a), se graficó la absorbancia medida como una función de la concentración de $\mathrm{H}_{2} \mathrm{O}_{2}(\mu \mathrm{M})$ y se obtuvo una correlación lineal entre ambos parámetros en el intervalo de trabajo (valores de absorbancia hasta 0,7$)$. De la curva de calibración $\left(\Delta \mathrm{A}^{505}\right.$ vs. $\left[\mathrm{H}_{2} \mathrm{O}_{2}\right]$ ) (Figura 5.8.b) se obtuvo el valor de la pendiente (F), el cual se utilizó para transformar los valores de $\mathrm{A}^{505}$ de las muestras estudiadas en valores de concentración molar de $\mathrm{H}_{2} \mathrm{O}_{2}$. 

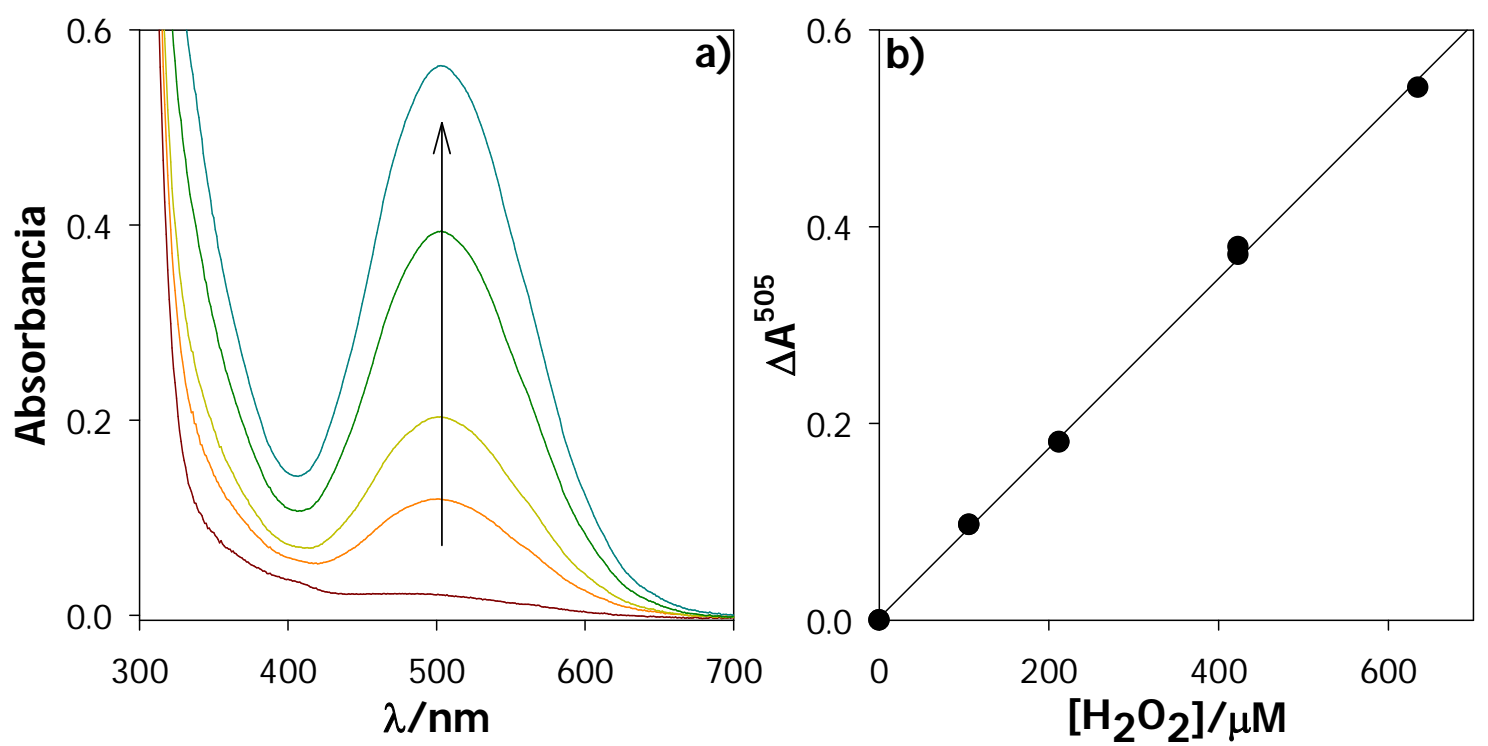

Figura 5.8 a) Espectros de absorción del complejo coloreado correspondiente a soluciones de $\mathrm{H}_{2} \mathrm{O}_{2}$ crecientes $(0,106,212,423,635 \mu \mathrm{M})$. b) Curva de calibración $\left(\Delta A^{505}\right.$ vs. $\left.\left[\mathrm{H}_{2} \mathrm{O}_{2}\right]\right)$.

\subsection{Medida electroquímica de oxígeno}

Para estudiar si el $\mathrm{O}_{2}$ participa en las reacciones fotoquímicas estudiadas, se realizaron determinaciones electroquímicas de concentración del $\mathrm{O}_{2}$ disuelto en las soluciones irradiadas. Para esto, se utilizó un electrodo sensible a $\mathrm{O}_{2}$ (Orion, modelo 37 08-99). La solución a irradiar se coloca en una celda de vidrio pyrex que contiene $120 \mathrm{ml}$ de solución y se cierra hidráulicamente al colocar el electrodo. Este dispositivo permite irradiar la solución y cuantificar el $\mathrm{O}_{2}$ disuelto en la solución al mismo tiempo. El electrodo posee en su extremo un buzo magnético, para homogeneizar la solución y se puede colocar otro buzo en el fondo de la celda. Por debajo se coloca un agitador magnético, para que las soluciones sean agitadas durante todo el experimento. Además, la membrana del electrodo está protegida de la luz UV durante la irradiación por un dispositivo plástico (Figura 5.9.a).

Antes de empezar la irradiación se realiza la medida de la concentración de $\mathrm{O}_{2}$ disuelto en la solución. Cabe destacar que, en todos los experimentos, se emplearon soluciones iniciales saturadas en aire. Finalmente, se inicia el experimento de fotólisis estacionaria y, sin apagar la lámpara, se efectúan las medidas a distintos tiempos de irradiación. Estos experimentos no pueden compararse cuantitativamente con las demás fotólisis realizadas en celdas de cuarzo, debido a que las condiciones y la geometría son diferentes.

La solubilidad del $\mathrm{O}_{2}$ en agua tiene una gran dependencia con la temperatura. Por lo tanto, para descartar que la disminución de la concentración de $\mathrm{O}_{2}$ sea debido al 
aumento en la temperatura por calentamiento provocado por la lámpara, se realizaron experimentos control irradiando $\mathrm{H}_{2} \mathrm{O}$. El resultado se muestra en la Figura 5.9.b, donde se observa un leve descenso de la señal al principio y luego la misma se mantiene. Esta disminución es despreciable frente a los resultados obtenidos en las distintas fotólisis.
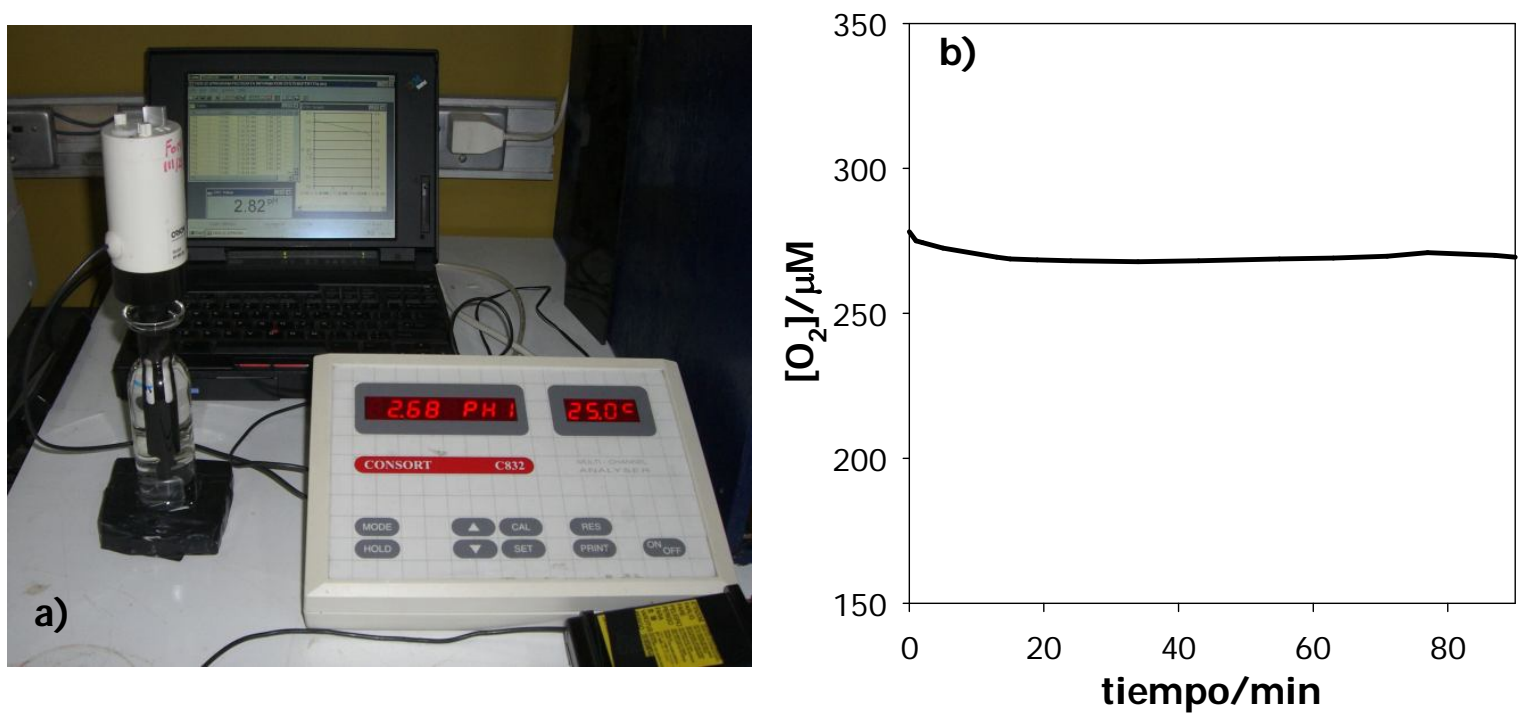

Figura 5.9 a) Fotografía del equipamiento utilizado para la medida electroquímica de $\mathrm{O}_{2}$ b) Medida de la concentración de $\mathrm{O}_{2}$ en $\mathrm{H}_{2} \mathrm{O}$ vs. tiempo de irradiación. Las condiciones y geometría fueron las mismas que en las fotólisis realizadas.

\subsection{Detección de anión superóxido por Citocromo c}

Para investigar la formación del $\mathrm{O}_{2}{ }^{\cdot-}$ a partir de ciertas reacciones fotoquímicas, se utilizó el método de reducción del Citocromo c (Cyt) inhibible por Superóxido Dismutasa (SOD). ${ }^{11}$ Durante este procedimiento, el Cyt captura un electrón del $\mathrm{O}_{2}{ }^{*-}$ y se reduce de ferricitocromo $\mathrm{c}\left(\mathrm{Fe}^{+3}\right)$ a ferrocitocromo $\mathrm{c}\left(\mathrm{Fe}^{+2}\right)$, modificándose el espectro de absorción, en el cual aumenta la absorbancia a $550 \mathrm{~nm}\left(\mathrm{~A}^{550}\right)$ tal como se observa en la Figura 5.10.a. A partir de estos espectros se obtiene un espectro diferencia típico que se encuentra publicado en literatura (Figura 5.10.b). ${ }^{12}$ En aquellos sistemas en los cuales se observa reducción del Cyt, se repite el ensayo en exactamente las mismas condiciones experimentales pero agregando SOD. Esta enzima es capaz de dismutar el $\mathrm{O}_{2}{ }^{\bullet-}$ en $\mathrm{H}_{2} \mathrm{O}_{2}$ y $\mathrm{O}_{2}$, eliminando el radical que reduce al Cyt, e inhibiendo así la reacción. ${ }^{13}$ Por lo tanto, se confirma la existencia de $\mathrm{O}_{2}{ }^{*-}$ solo si se observa la inhibición o disminución de la reducción del Cyt.

De este modo, el ensayo es muy útil para determinar reacciones de transferencia de electrones generadas a partir de $\mathrm{O}_{2}{ }^{*-}$ (en soluciones aireadas). ${ }^{14}, 15$ Sin embargo, también se puede observar la reducción del Cyt en condiciones anaeróbicas, donde la transferencia de electrones es directamente a partir del sensibilizador, y no se aprecia 
inhibición cuando se agrega SOD. Esto fue observado en el caso de benzo[a]pireno,, ${ }^{16}$ antraceno, ${ }^{17}$ entre otros.

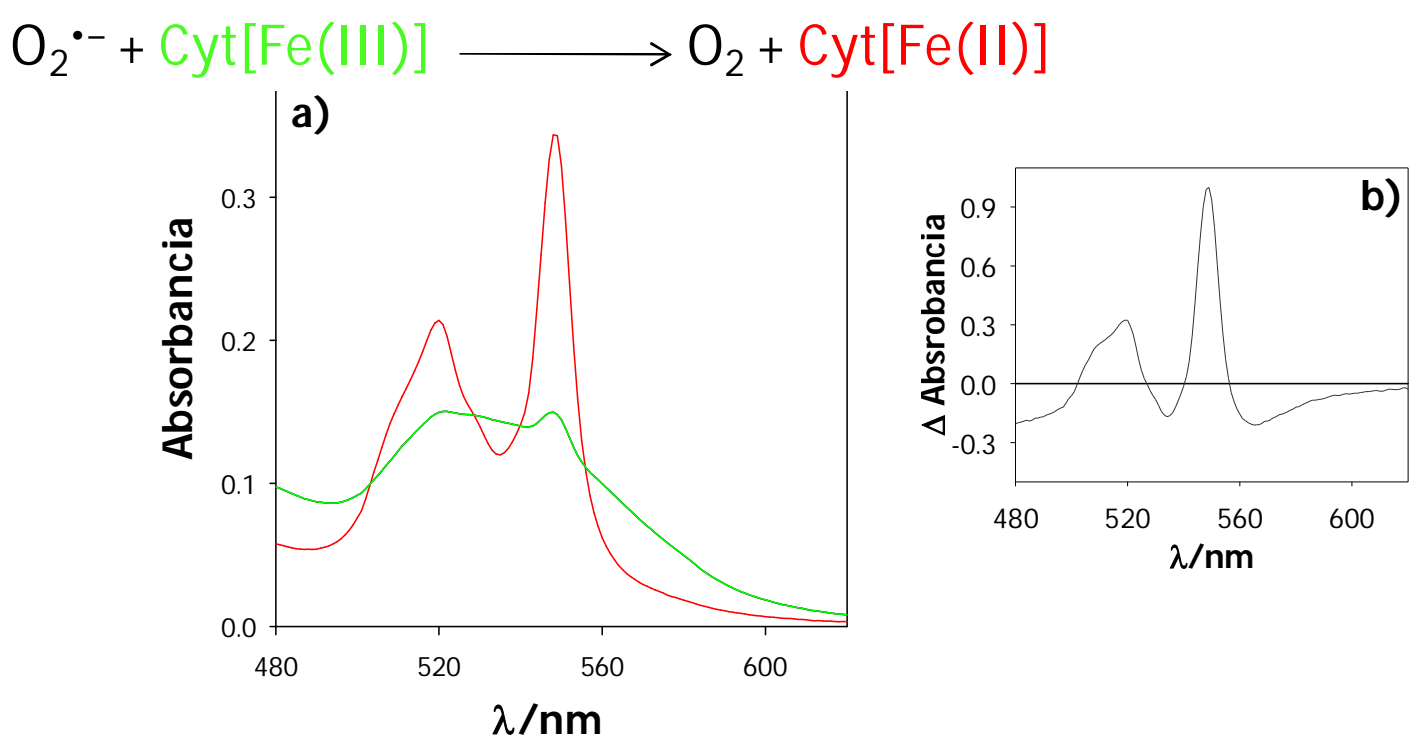

Figura 5.10 a) Espectros de absorción de Cyt c oxidado (línea verde) y reducido (línea roja), b) Espectro diferencia típico entre las formas reducida y oxidada del Cyt.

Los experimentos se realizaron irradiando soluciones aireadas de las pterinas oxidadas (75 $\mu \mathrm{M})$ en presencia de $14 \mu \mathrm{M}$ de Cyt, a pH ácido y alcalino. Se registraron los espectros de absorción a distintos intervalos de tiempo y luego se graficó la $\mathrm{A}^{550}$ en función del tiempo de irradiación. Para confirmar que el $\mathrm{O}_{2}{ }^{-}$era el responsable de la reducción del Cyt se agregó SOD (200 U/ml). Además, se llevaron a cabo experimentos en condiciones de anaerobiosis para detectar si el Cyt era reducido directamente por el sensibilizador, como se explicó anteriormente.

\subsection{Referencias}

1. C. Chahidi, M. Aubailly, A. Momzikoff, M. Bazin and R. Santus, Photophysical and photosensitizing propierties of 2-amino-4 pteridinone: a natural pigment, Photochemistry and Photobiology, 1981, 33, 641-649.

2. K. V. Neverov, E. A. Mironov, T. A. Lyudnikova, A. A. Krasnovskij and M. S. Kritskij, Phosphorescence analysis of triplet state of pterins in connection with their photoreceptor function in biochemical systems, Biokhimiya, 1996, 61, 1627-1636.

3. J. A. Weil, J. R. Bolton and J. E. Wertz, Electron Paramagnetic Resonance. Elementary Theory and Practical Applications, John Wiley \& Sons, New York 1994.

4. A. Rizzi, Estudio de Interacciones Magnéticas y Estructura Electrónica en Complejos de Metales de Transición con Moléculas de Interés Biológico, Universidad Nacional del Litoral, Santa Fe, 2003.---

5. E. G. Janzen, Spin trapping, Accounts of Chemical Research, 1971, 4, 31-40.

6. F. A. Villamena and J. L. Zweier, Detection of Reactive Oxygen and Nitrogen Species EPR Spin Trapping, Antioxidants and Redox Signaling, 2004, 6, 619-629.

7. F. A. Villamena, Superoxide radical anion adduct of 5,5-dimethyl-1-pyrroline N-Oxide. 5. thermodynamics and kinetics of unimolecular decomposition, Journal of Physical Chemistry A, 2009, 113, 6398-6403.

8. I. Fridovich, Superoxide radicals, superoxide dismutases and the aerobic lifestyle, Photochemistry and Photobiology, 1978, 28, 733-741. 
9. E. Finkelstein, G. M. Rosen and E. J. Rauckman, Spin trapping. Kinetics of the reaction of superoxide and hydroxyl radicals with nitrones, Journal of American Chemistry Society, 1980, 102, 4994-4999.

10. C. Hadjur, G. Wagnières, F. Ihringer, P. Monnier and H. Van Den Bergh, Production of the free radicals $\mathrm{O}_{2}$ *- and ' $\mathrm{OH}$ by irradiation of the photosensitizer zinc (II) phthalocyanine, Journal of Photochemistry and Photobiology B: Biology, 1997, 38, 196-202.

11. D. E. Moore and J. Wang, Electron-transfer mechanisms in photosensitization by the antiinflammatory drug benzydamine, J. Photochem. Photobiol. B, 1998, 43, 175-180.

12. R. Kuciel and A. Mazurkiewicz, Formation and detoxification of reactive oxygen species, Biochemistry and Molecular Biology Education, 2004, 32, 183-186.

13. J. M. McCord and I. Fridovich, The Utility of Superoxide Dismutase in Studying Free Radical Reactions. I. Radiclas generated by the interaction of sulfite, dimethyl sulfoxide, and oxygen, J. Biol. Chem., 1969, 244, 6056-6063.

14. S. D. Picker and I. Fridovich, On the mechanism of production of superoxide radical by reaction mixtures containing $\mathrm{NADH}$, phenazine methosulfate, and nitroblue tetrazolium, Archives of Biochemistry and Biophysics, 1984, 228, 155-158.

15. T. W. Kirby and I. Fridovich, A picomolar spectrophotometric assay for superoxide dismutase, Analytical Biochemistry, 1982, 127, 435-440.

16. J. Kagan, M. Bazin and R. Santus, Photosensitization with [alpha]-terthienyl: The formation of superoxide ion in aqueous media, Journal of Photochemistry and Photobiology B: Biology, 1989, 3, 165-174.

17. R. W. Tuveson, G.-R. Wang, T. P. Wang and J. Kagan, Light-dependent Cytotoxic Reactions of Anthracene, Photochemistry and Photobiology, 1990, 52, 993-1002. 


\section{PARTE III}

\section{RESULTADOS}





\section{Capítulo 6}

\section{Fotoquímica de biopterina y neopterina}

En este capítulo se presentarán los resultados del estudio de la fotoquímica de las pterinas oxidadas biopterina (Bip) y neopterina (Nep). Ambas exhiben un comportamiento muy similar, motivo por el cual se discutirán de manera conjunta. En particular, se obtuvieron los rendimientos cuánticos de consumo de reactivo, se identificaron y caracterizaron los productos de reacción, se estudió la producción de especies reactivas de oxígeno (EROs) y se analizaron los mecanismos de reacción, en distintas condiciones experimentales.

Se trabajó en dos condiciones de $\mathrm{pH}$ : medio ácido $(\mathrm{pH}=5,5)$ y medio alcalino ( $\mathrm{pH}$ $=10,5)$, para contar con una especie predominante según el equilibrio ácido-base, tal como se explicó en el Capítulo 2. Además, se evaluó el comportamiento de Bip y Nep en distintas atmósferas (soluciones aireadas, saturadas en oxígeno o saturadas en argón) con el objeto de investigar la participación del oxígeno en las vías de fotooxidación.

\subsection{Fotólisis en presencia de oxígeno}

Soluciones acuosas de Bip y Nep (100 - $160 \mu \mathrm{M}, \mathrm{pH}=5,5$ y 10,5) fueron irradiadas con el Sistema de irradiación I (Sección 4.3) durante distintos intervalos de tiempo (tiempo de irradiación, ti). Como se observa en la Figura 6.1, se produjeron cambios característicos en los espectros de absorción, los cuales fueron muy similares para ambas pterinas. Esencialmente, la banda de mayor energía disminuye su intensidad, mientras que la de menor energía aumenta. Además, para ambas pterinas se detecta la presencia de los mismos puntos isosbésticos (a $285 \mathrm{~nm}$ en medio ácido y a $265 \mathrm{~nm}$ medio alcalino) que se mantienen invariables por más de 5 min de irradiación, sugiriendo la existencia de un único proceso fotoquímico. Esto se aprecia mejor al observar los espectros diferencias experimentales (EDE) (insets en la Figura 6.1). Es importante destacar que no se observaron cambios en los espectros cuando las soluciones irradiadas se almacenaron en la oscuridad por varias horas. Es decir, que los fotoproductos, en principio, son estables en ausencia de irradiación. 


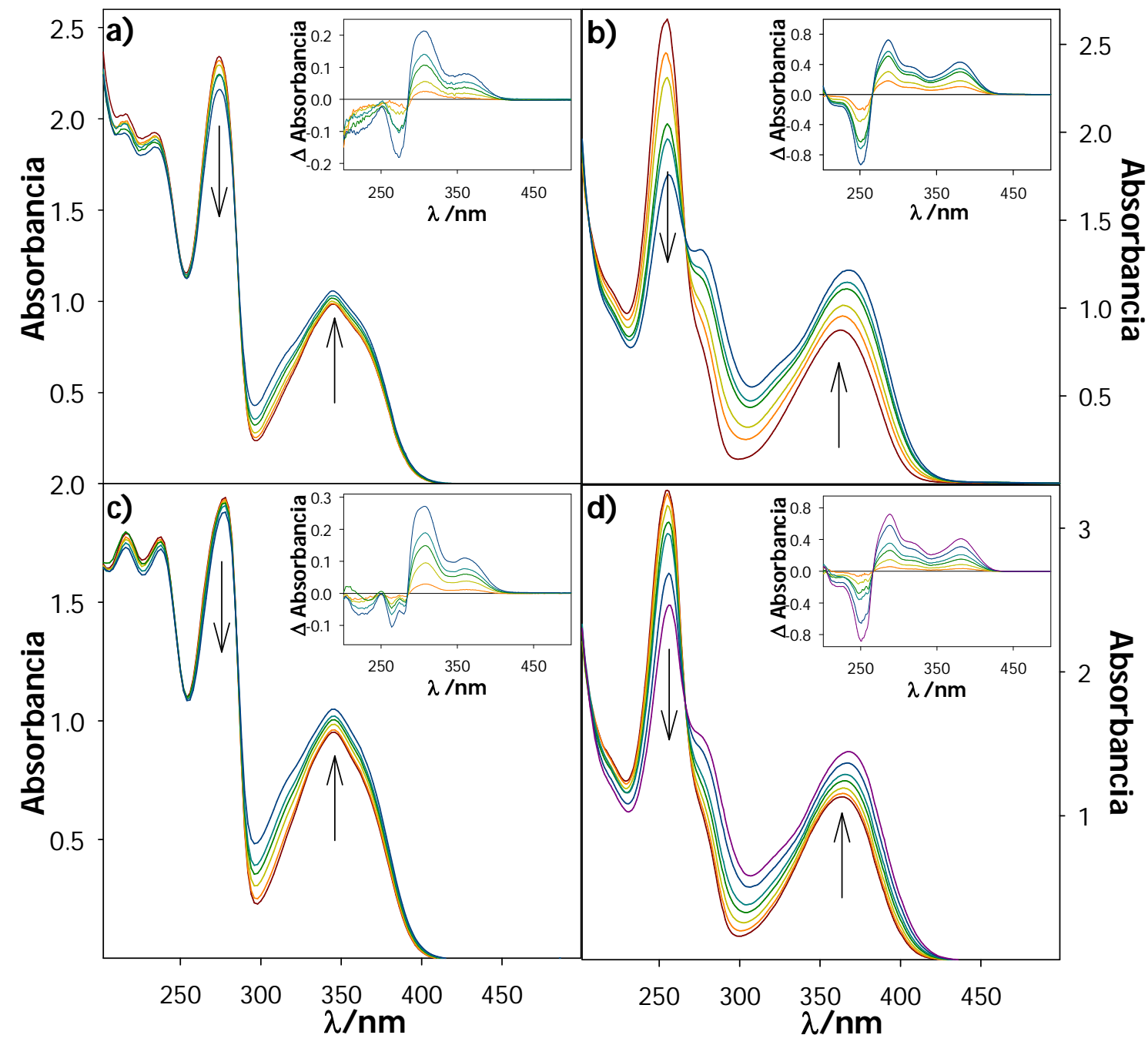

Figura 6.1 Evolución de los espectros de absorción en función del tiempo de irradiación en soluciones aireadas. a) [Bip] $]_{0}=150 \mu \mathrm{M}, \mathrm{pH}=5,5, \mathrm{ti}=0,30,60,90,120,180 \mathrm{~s} ; \mathrm{b}$ ) $[\mathrm{Bip}]_{0}$ $=118 \mu \mathrm{M}, \mathrm{pH}=10,6, \mathrm{ti}=0,30,60,90,120,150 \mathrm{~s}$; $\mathrm{c}$ [ $[\mathrm{Nep}]_{0}=150 \mu \mathrm{M}, \mathrm{pH}=5,3, \mathrm{ti}=0,30$, $60,90,120,180 \mathrm{~s} ; \mathrm{d}$ ) $[\mathrm{Nep}]_{0}=148 \mu \mathrm{M}, \mathrm{pH}=10,6, \mathrm{ti}=0,15,30,45,60,90,120 \mathrm{~s}$. Camino óptico $=1 \mathrm{~cm}$. Sistema de irradiación I. Insets: EDE.

Como se detalló en el Capítulo 3, trabajos previos demostraron que cuando Bip o Nep son irradiadas en presencia de $\mathrm{O}_{2}$, generan, como producto estable, 6-formilpterina (Fop).1-4 Teniendo en cuenta esta reacción fotoquímica, se comparó el espectro diferencia experimental normalizado (EDEN) obtenido a partir de la diferencia entre el espectro de absorción de una solución de Bip irradiado un ti determinado y el espectro sin irradiar, con el espectro diferencia teórico normalizado (EDTN) obtenido restando el espectro patrón de Bip al espectro patrón de Fop, ambos registrados en las mismas condiciones de pH. De la misma manera, se compararon los espectros EDEN de las fotólisis de Nep con los correspondientes espectros EDTN obtenidos a partir de soluciones patrón de Fop y Nep. Los detalles del procedimiento empleado para obtener los espectros EDEN y EDTN se explicaron en el Capítulo 5. En la Figura 6.2, se observa 
claramente que los EDEN se aproximan a los correspondientes EDTN, sugiriendo que, en ambas condiciones de $\mathrm{pH}$ y para las dos pterinas oxidadas estudiadas, el principal producto de fotólisis es Fop.
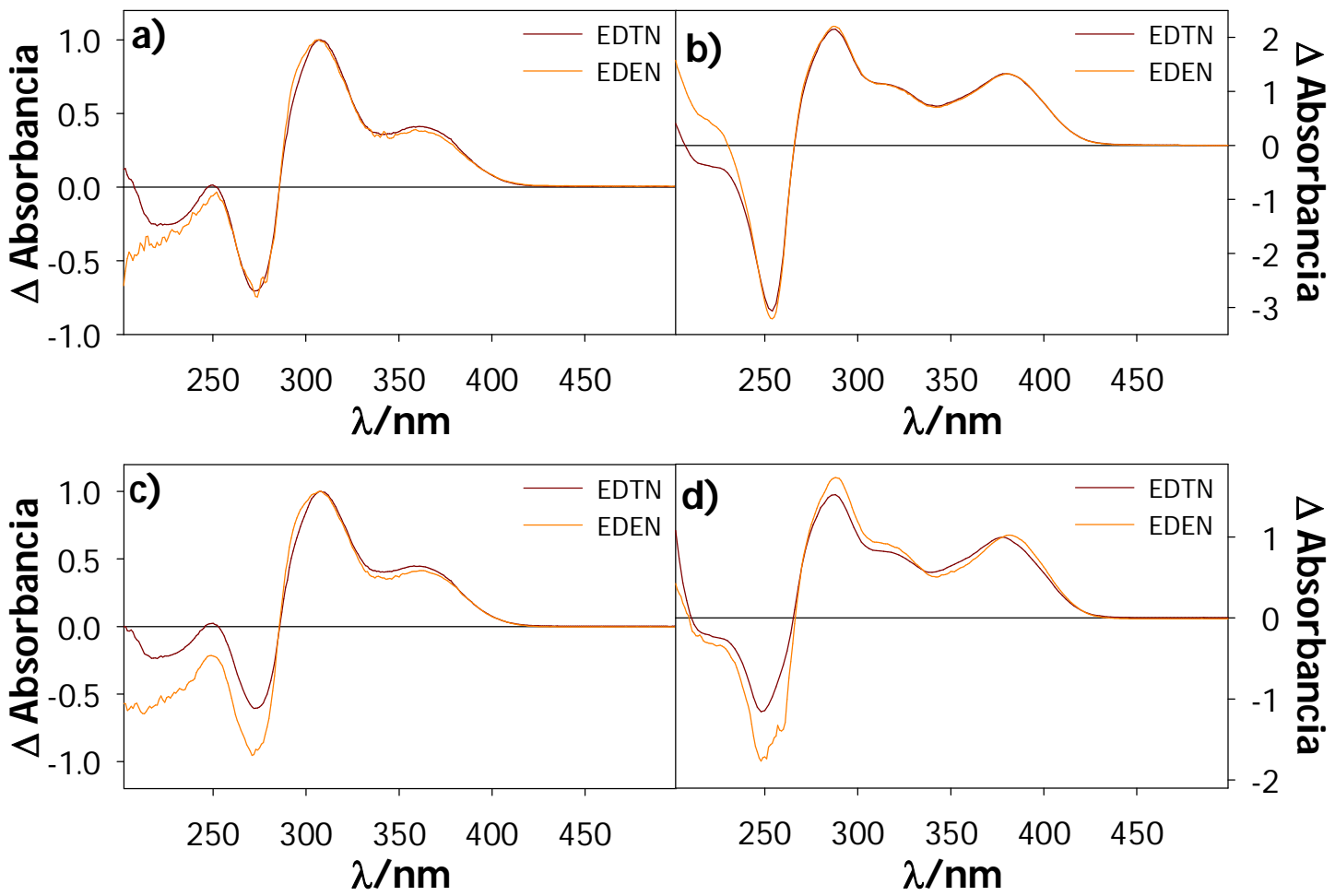

Figura 6.2 Comparación de espectros EDEN de soluciones irradiadas en aire con espectros EDTN correspondientes a reacciones en los cuales Fop es el producto de fotólisis. a) Bip, $\mathrm{pH}=5,4$; b) Bip, $\mathrm{pH}=10,5$; c) Nep, $\mathrm{pH}=5,5$; d) Nep, $\mathrm{pH}=10,6$.

Las soluciones irradiadas fueron luego analizadas por HPLC. En la Figura 6.3 se muestran los cromatogramas (longitud de onda de análisis, $\lambda_{\text {an }}=340 \mathrm{~nm}$ ) correspondientes a una solución de Bip $(118 \mu \mathrm{M}, \mathrm{pH}=10,6)$ irradiada distintos intervalos de tiempo. El pico principal en la solución no irradiada corresponde al reactivo (tiempo de retención, tr $=4,7 \mathrm{~min}$ ), el cual va disminuyendo su área a medida que aumenta el tiempo de irradiación. A su vez, aparece, en primera instancia, un pico ancho con $\operatorname{tr}=7,2$ min y luego, otro de menor área con $\operatorname{tr}=2,1$ min. Este último producto se genera recién después de que se acumula el primer producto. Comparando los tiempos de retención y los espectros de absorción, (registrados con el detector del HPLC) con el de los compuestos patrones, se concluyó que los productos formados son Fop y 6-carboxipterina (Cap). Resultados similares fueron obtenidos al irradiar soluciones de Nep en ambas condiciones de $\mathrm{pH}$. 

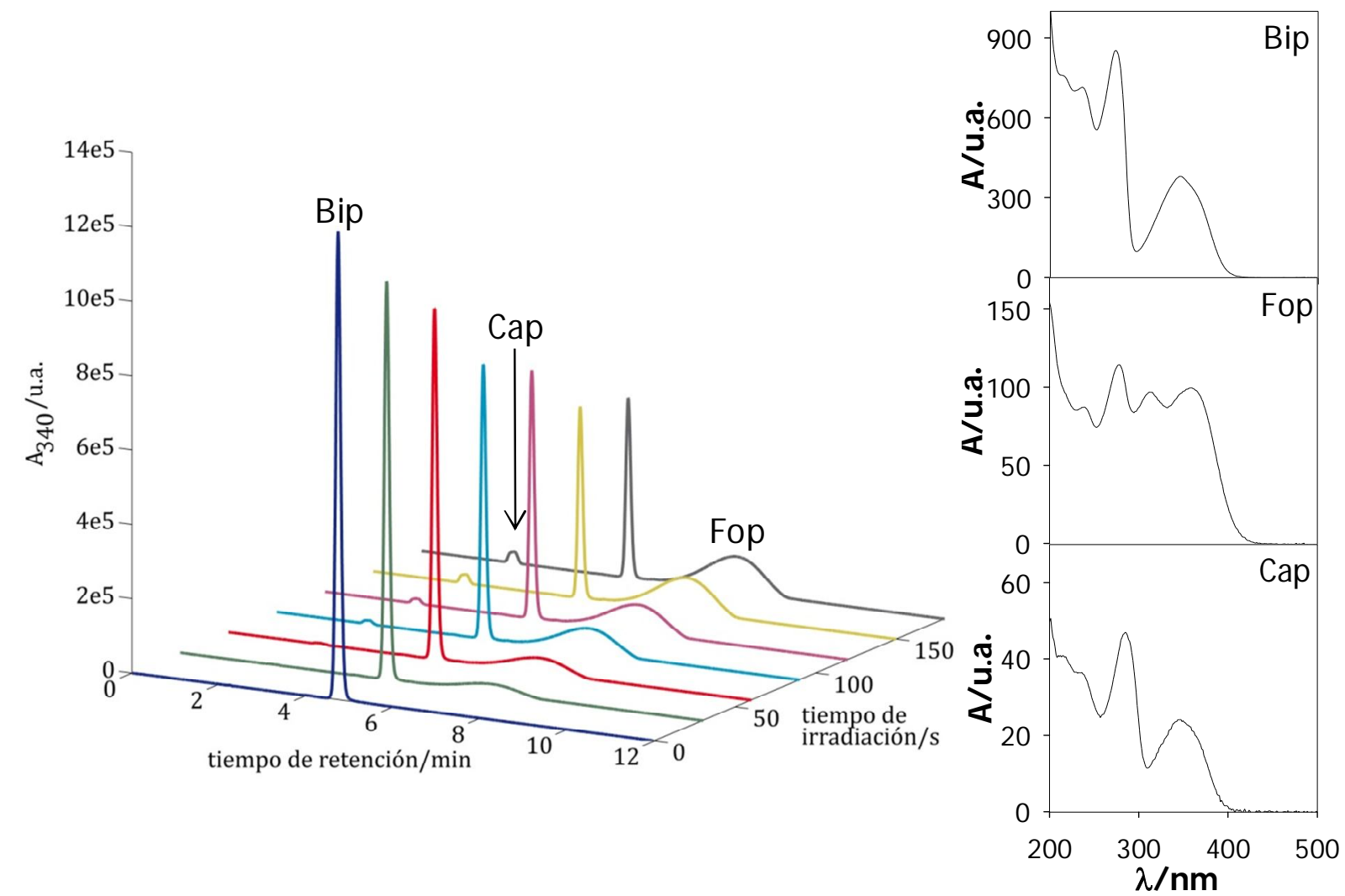

Figura 6.3 Cromatogramas $\left(\lambda_{\mathrm{an}}=340 \mathrm{~nm}\right)$ obtenidos durante la irradiación de una solución de Bip en aire $\left([B i p]_{0}=118 \mu \mathrm{M}, \mathrm{pH}=10,6\right)$. Espectros de absorción del reactivo (Bip) y los fotoproductos (Fop y Cap) obtenidos con el detector del HPLC. Sistema de irradiación I y Equipo HPLC I.

Con los valores de las áreas obtenidas a partir de los cromatogramas y las correspondientes curvas de calibración (Capítulo 5) se realizaron los perfiles de concentración de reactivos y productos en función del tiempo de irradiación (Figura 6.4). Se puede observar que el consumo de los reactivos y la formación del producto principal siguen una ley de velocidad de orden cero, al menos durante los primeros 3 min de irradiación. Esto es importante para determinar el rendimiento cuántico, como se detalla en la Sección 4.4. Además, se aprecia que en todos los casos, la suma de las concentraciones del reactivo y los productos, a cada tiempo de irradiación, permanecen constantes durante todo el experimento, indicando que Fop y Cap son los principales fotoproductos.

De acuerdo a los antecedentes en literatura expuestos en el Capítulo 3, Cap es el producto de la oxidación fotoquímica de Fop. ${ }^{5}$ Por lo tanto, a tiempos largos de fotólisis de Bip o Nep, cuando se acumula Fop, este compuesto también comienza a consumirse, generando Cap como producto final. Esto se aprecia más claramente en la Figura 6.5.a, donde se exponen como ejemplo, los perfiles de concentración de una solución de Bip en una ventana de tiempo más prolongado (10 min) a las expuestas anteriormente. 


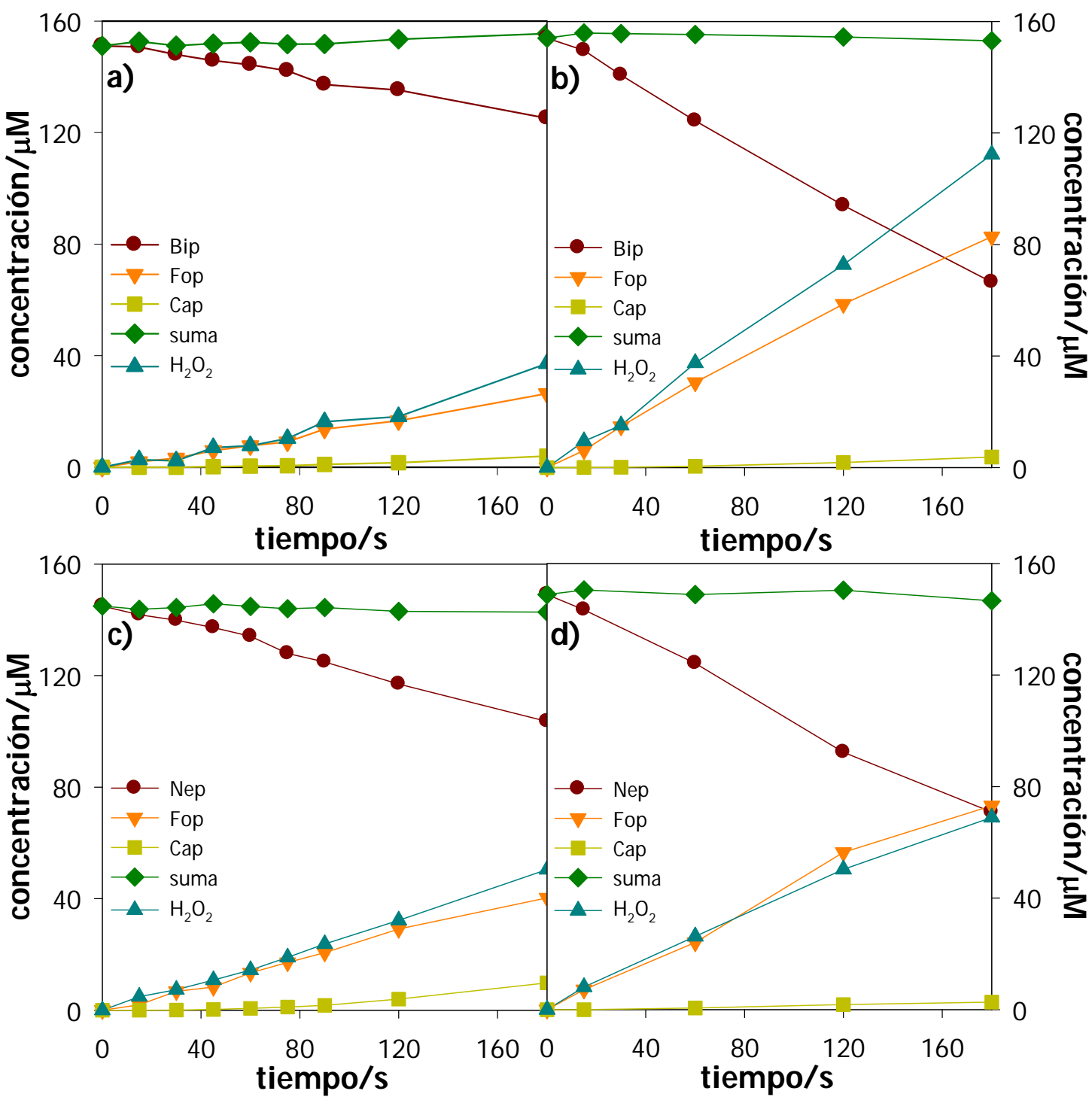

Figura 6.4 Perfiles de concentración de reactivos y productos para soluciones irradiadas en presencia de $\mathrm{O}_{2}$. a) $\left.[\mathrm{Bip}]_{0}=150 \mu \mathrm{M}, \mathrm{pH}=5,5 ; \mathrm{b}\right)[\mathrm{Bip}]_{0}=155 \mu \mathrm{M}, \mathrm{pH}=10,4 ;$ c) $[\mathrm{Nep}]_{0}=$ $145 \mu \mathrm{M}, \mathrm{pH}=5,6 ;$ d) $[\mathrm{Nep}]_{0}=150 \mu \mathrm{M}, \mathrm{pH}=10,5$. Suma, corresponde a la suma de concentraciones de reactivo (Bip o Nep), Fop y Cap, a cada tiempo de irradiación. Sistema de irradiación I y Equipo HPLC I.

Asimismo se evaluó la producción de $\mathrm{H}_{2} \mathrm{O}_{2}$ (Sección 5.7) en las soluciones irradiadas de Bip y Nep. Como se observa en la Figura 6.4, la concentración de $\mathrm{H}_{2} \mathrm{O}_{2}$ se incrementa a medida que avanza el tiempo de irradiación. Se observa que, a tiempos de irradiación relativamente cortos, la concentración de $\mathrm{H}_{2} \mathrm{O}_{2}$ encontrada fue similar a la concentración de Fop producida. Sin embargo, a tiempos más largos, $\mathrm{H}_{2} \mathrm{O}_{2}$ formado fue mayor que la concentración de Fop e, incluso, mayor que la concentración de reactivo consumido. Esto se debe a la fotooxidación de Fop para dar Cap y $\mathrm{H}_{2} \mathrm{O}_{2}{ }^{6}$ comentada previamente. Es decir que, a un tiempo determinado, $\left[\mathrm{H}_{2} \mathrm{O}_{2}\right]=[\mathrm{Fop}]+2[\mathrm{Cap}]$. Esto se aprecia claramente en la Figura 6.5.b. 

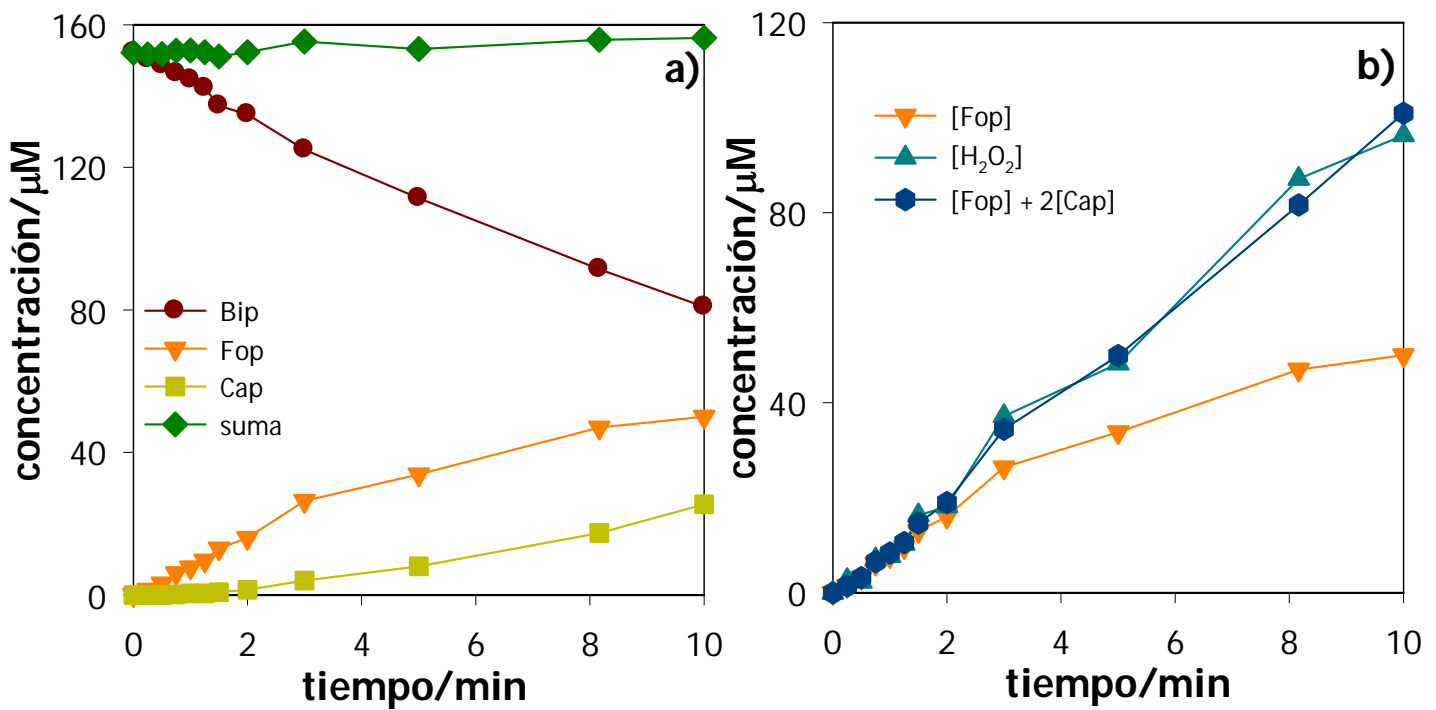

Figura 6.5 a) Perfiles de concentración de reactivo y productos para soluciones irradiadas en presencia de $\mathrm{O}_{2}$. [Bip $]_{0}=153 \mu \mathrm{M}, \mathrm{pH}=5,6$. Suma, corresponde a la suma de concentraciones de Bip, Fop y Cap, a cada tiempo de irradiación. b) Evolución de la concentración de $\mathrm{H}_{2} \mathrm{O}_{2}$ en el mismo sistema de reacción que a). Sistema de irradiación I y Equipo HPLCI.

Para continuar con el estudio, se analizó la evolución de la concentración de $\mathrm{O}_{2}$ durante la irradiación de una solución de Bip o Nep, monitoreada con un electrodo de $\mathrm{O}_{2}$ en celda cerrada. Estos experimentos fueron realizados con una geometría diferente a la de los experimentos descriptos hasta el momento, explicada en la Sección 5.8. Como se aprecia en la Figura 6.6, en ambas condiciones de $\mathrm{pH}$ y para las dos pterinas estudiadas, se observó una disminución significativa de la concentración de $\mathrm{O}_{2}$ con el tiempo de irradiación. Asimismo, se realizó un control en $\mathrm{H}_{2} \mathrm{O}$ y el consumo de dicho gas es despreciable frente a lo observado en las soluciones de Bip y Nep.

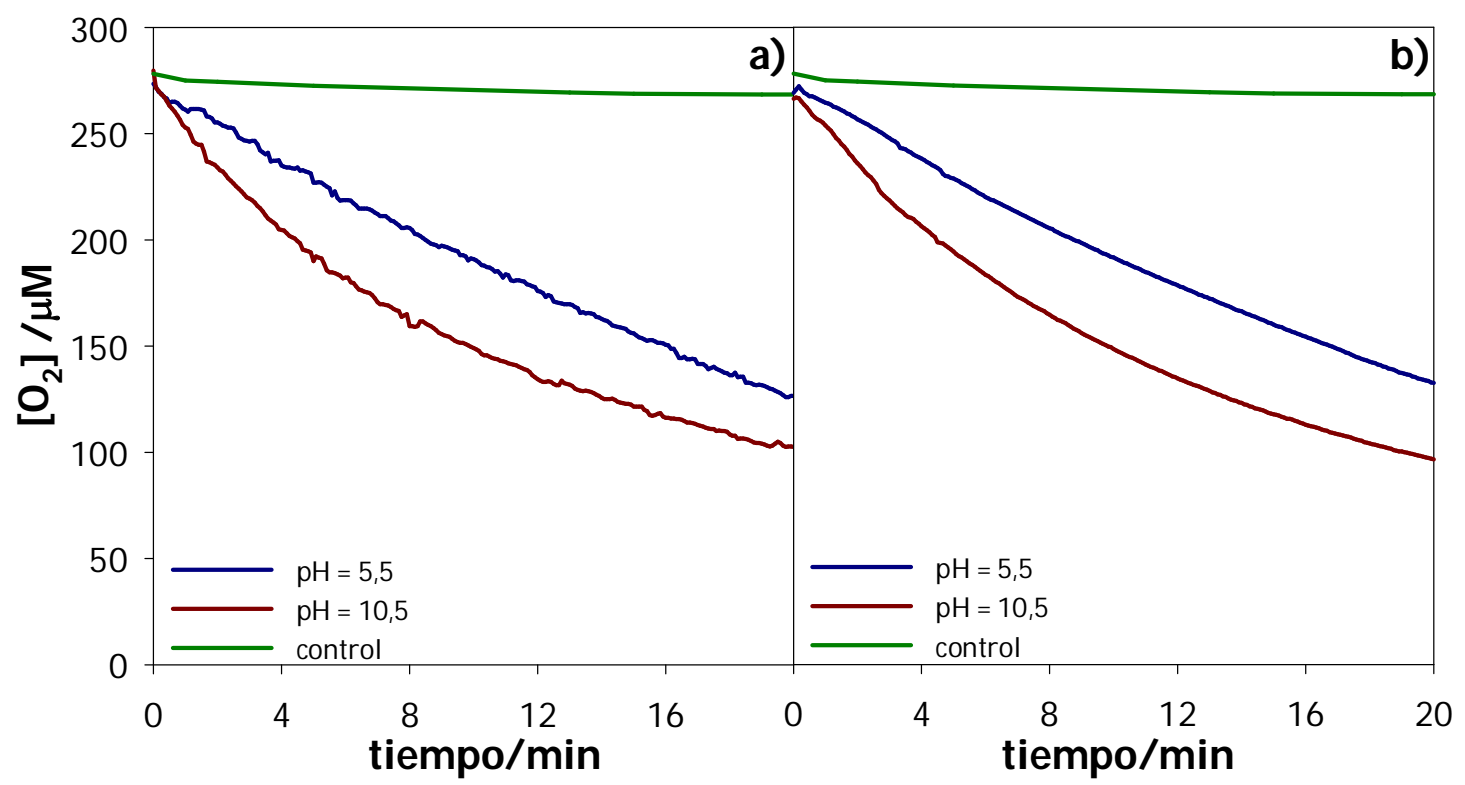

Figura 6.6 Evolución de la concentración de $\mathrm{O}_{2}$ disuelto con el tiempo de irradiación. a) $\left[\mathrm{Bip}_{0}=153 \mu \mathrm{M}\right.$; b) $[\mathrm{Nep}]_{0}=150 \mu \mathrm{M}$. Sistema de irradiación $\mathrm{I}$. 
En los experimentos de determinación de $\mathrm{O}_{2}$ consumido, al finalizar la irradiación de cada solución las muestras se analizaron por HPLC y se determinaron las concentraciones finales de reactivos y productos. Con estos datos se realizó un balance de masa que se muestra en la Tabla 6.1. Se observa que la concentración de $\mathrm{O}_{2}$ consumida es igual, dentro del error experimental, a la suma de la concentración de Fop generada más dos veces la concentración de Cap. Por lo tanto, esto sugiere que por cada molécula del reactivo fotooxidado se consume una molécula de $\mathrm{O}_{2}$, y que en la fotooxidación secundaria también se consume $\mathrm{O}_{2}$ con una estequiometría 1:1.

\begin{tabular}{|c|c|c|c|c|c|c|}
\hline & $\begin{array}{c}\text { ti } \\
(\min )\end{array}$ & $\begin{array}{l}\Delta[\mathrm{Pt}] \\
(\mu \mathrm{M})\end{array}$ & $\begin{array}{l}\text { [Fop] } \\
(\mu \mathrm{M})\end{array}$ & $\begin{array}{l}\text { [Cap] } \\
(\mu \mathrm{M})\end{array}$ & $\begin{array}{c}\text { Fop]+2[Cap] } \\
(\mu \mathrm{M})\end{array}$ & $\begin{array}{l}\Delta\left[\mathrm{O}_{2}\right] \\
(\mu \mathrm{M})\end{array}$ \\
\hline Bip & 18 & 100 & 57 & 33 & 123 & 124 \\
\hline pH5 & 27 & 134 & 58 & 62 & 182 & 188 \\
\hline Bip & 10 & 125 & 107 & 10 & 127 & 133 \\
\hline pH 10 & 22 & 147 & 110 & 25 & 160 & 175 \\
\hline Nep & 15 & 84 & 52 & 28 & 108 & 114 \\
\hline pH5 & 28 & 117 & 51 & 59 & 169 & 176 \\
\hline Nep & 9 & 98 & 77 & 6 & 89 & 111 \\
\hline pH 10 & 20 & 131 & 99 & 15 & 129 & 172 \\
\hline
\end{tabular}

Tabla 6.1 Comparación de consumo de reactivos y formación de productos. Para cada reactivo se muestran dos experimentos distintos.

Teniendo en cuenta los productos detectados, los perfiles de concentración y los balances de masa, se puede plantear la secuencia de reacciones que se detalla en el Esquema 6.1.

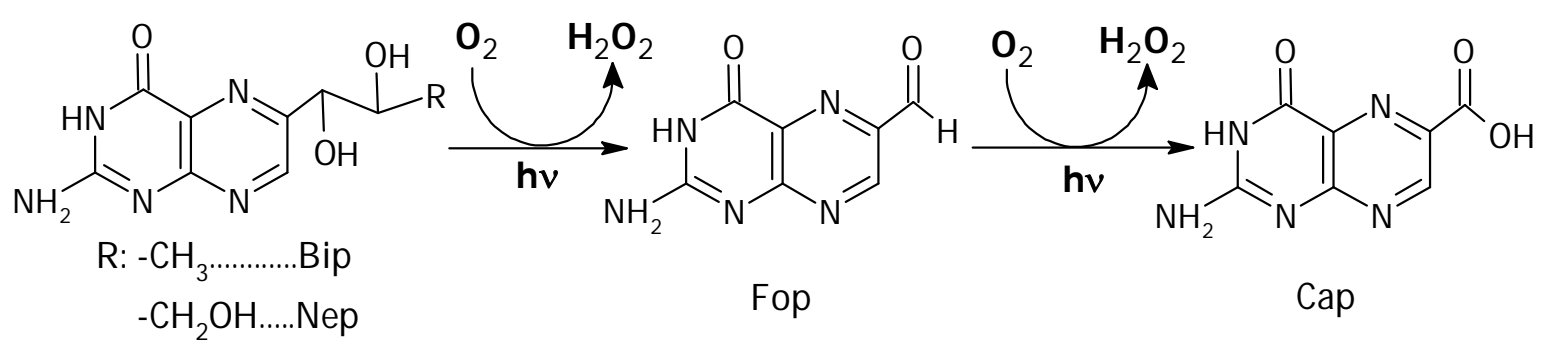

\section{Esquema 6.1 Fotooxidación de Bip y Nep.}

Por otro lado, se realizaron experimentos análogos a los ya descriptos, pero en soluciones saturadas en $\mathrm{O}_{2}$ (Sección 4.2.4). Las reacciones observadas fueron similares a las obtenidas en soluciones equilibradas en aire, donde Fop es el producto principal, Cap se forma en la reacción secundaria a partir de Fop, y $\mathrm{H}_{2} \mathrm{O}_{2}$ se forma estequiométricamente en las dos fotooxidaciones. Sin embargo, para cada reactivo (Bip o Nep) y en ambas condiciones de pH, la velocidad de oxidación fue menor en las soluciones saturadas en $\mathrm{O}_{2}$ que las observadas en las soluciones equilibradas en aire 
descriptas en los párrafos anteriores. En la Figura 6.7, se muestran los perfiles de concentración obtenidos a partir de las irradiaciones de soluciones de Bip y Nep saturadas en $\mathrm{O}_{2}$.
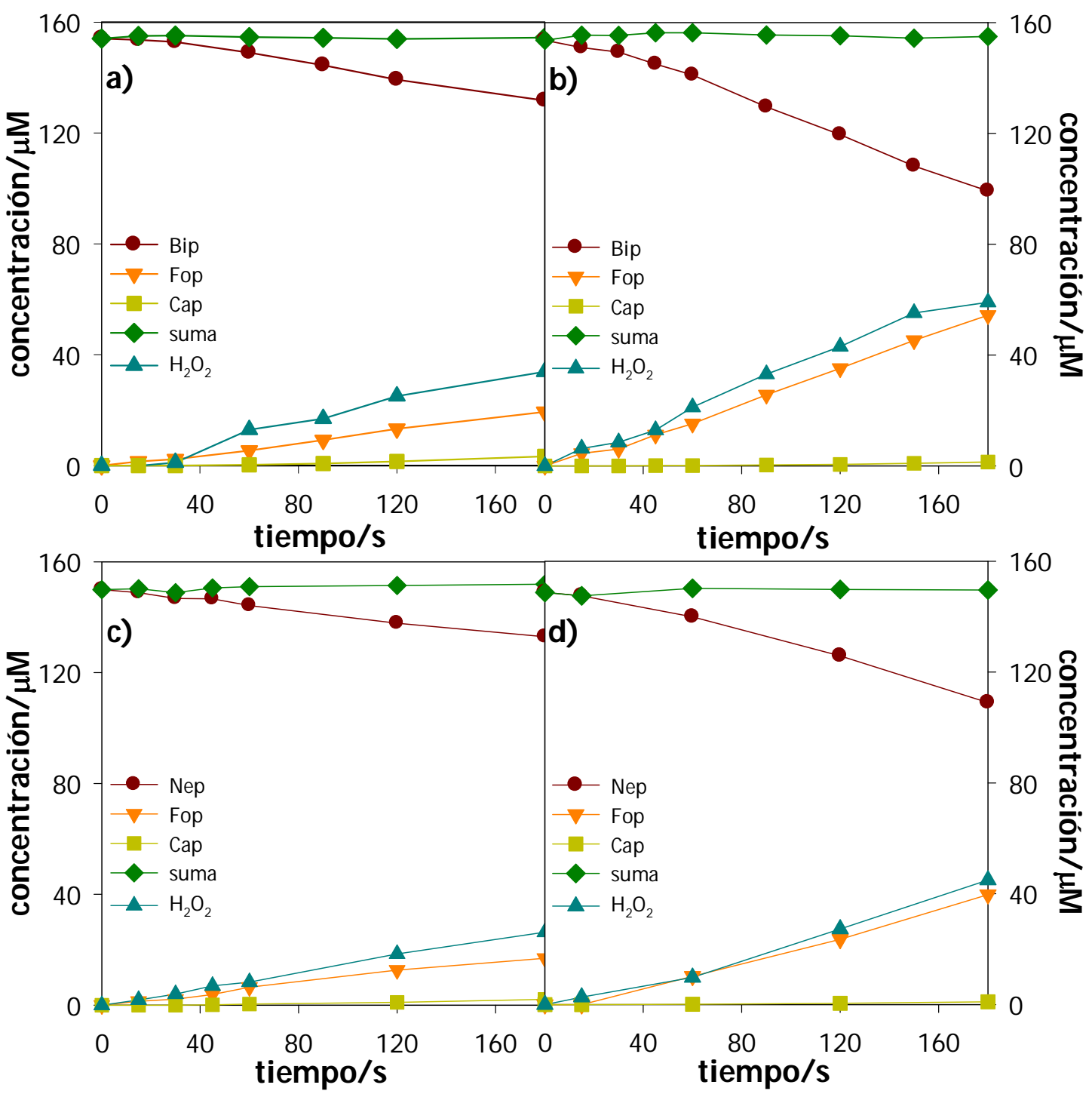

Figura 6.7 Perfiles de concentración de reactivos y productos para soluciones saturadas en $\mathrm{O}_{2}$. a) $\left.\left.[\mathrm{Bip}]_{0}=149 \mu \mathrm{M}, \mathrm{pH}=5,5 ; \mathrm{b}\right)[\mathrm{Bip}]_{0}=150 \mu \mathrm{M}, \mathrm{pH}=10,6 ; \mathrm{c}\right)[\mathrm{Nep}]_{0}=149 \mu \mathrm{M}, \mathrm{pH}=$ 5,$7 ;$ d) $[\mathrm{Nep}]_{0}=149 \mu \mathrm{M}, \mathrm{pH}=10,6$. Suma, corresponde a la suma de concentraciones de reactivo (Bip o Nep), Fop y Cap, a cada tiempo de irradiación. Sistema de irradiación I y Equipo HPLCI.

Las reacciones estudiadas son fotooxidaciones que, tal como se mostró anteriormente, consumen $\mathrm{O}_{2}$ disuelto. Sin embargo, al aumentar la concentración de $\mathrm{O}_{2}$ la velocidad de fotólisis disminuye. Esto se aprecia claramente en la Figura 6.8, donde se compara una solución aireada de Bip en medio ácido con otra en condiciones de saturación de $\mathrm{O}_{2}$, ambas irradiadas en las mismas condiciones. Este comportamiento inesperado, observado para ambos compuestos en los dos medios, puede deberse a la participación de estados excitados tripletes los cuales son, generalmente, desactivados 
con gran eficiencia por el $\mathrm{O}_{2}$ disuelto. Este punto se discutirá con mayor detalle más adelante en este capítulo.

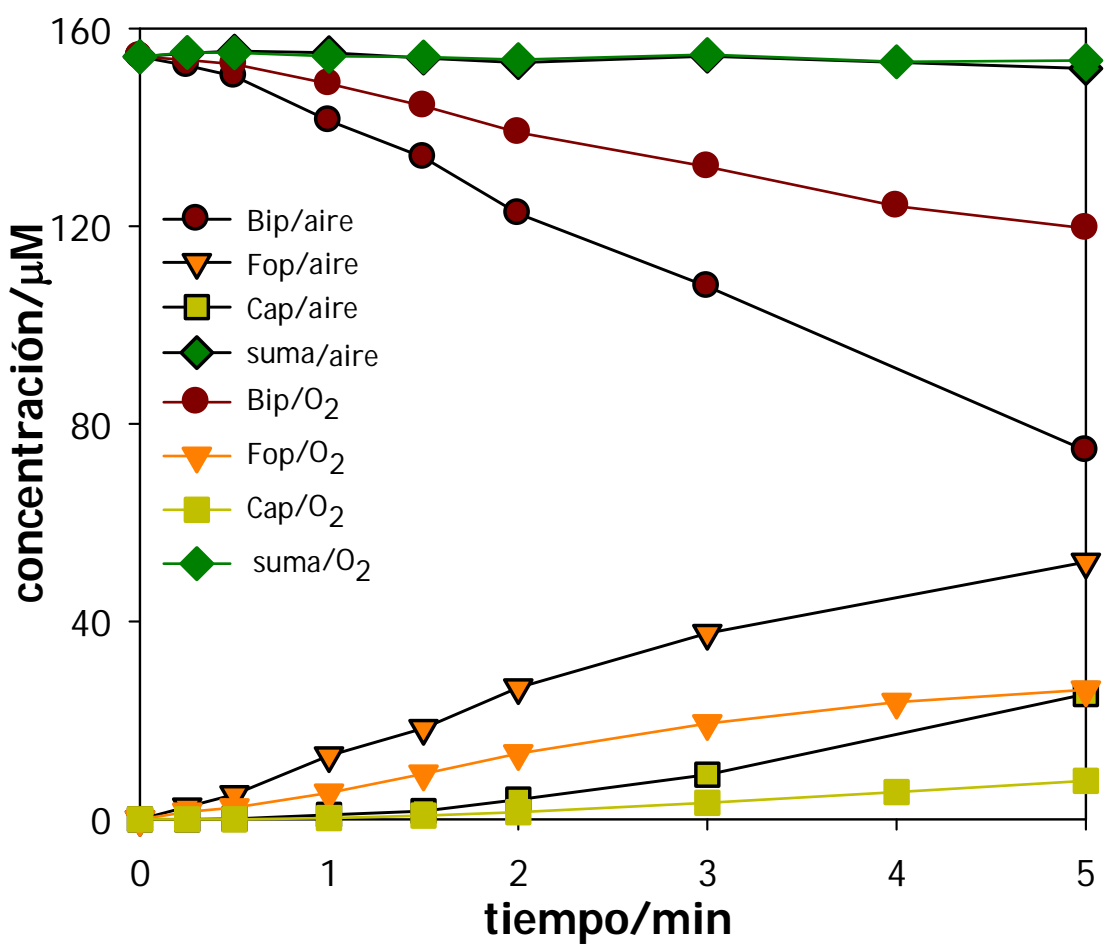

Figura 6.8 Perfiles de concentración de reactivos y productos para soluciones de Bip irradiadas en aire y saturadas en $\mathrm{O}_{2}$. [Bip $]_{0}=154 \mu \mathrm{M}, \mathrm{pH}=5,5$. Suma, corresponde a la suma de concentraciones de Bip, Fop y Cap, a cada tiempo de irradiación. Sistema de irradiación I y Equipo HPLC I.

\subsection{Fotólisis en ausencia de oxígeno}

Se realizaron irradiaciones en medio ácido y alcalino de soluciones acuosas de Bip y Nep, previa saturación en Ar, eliminando así el $\mathrm{O}_{2}$ disuelto (Sección 4.2.4). Como se observa en la Figura 6.9, los espectros de absorción varían con el tiempo de irradiación. Disminuye la banda de menor energía y aparece una banda de absorción, ancha y de baja intensidad, centrada en $480 \mathrm{~nm}$. Esto se percibe a simple vista ya que la solución se torna de color rojo. Al airear las soluciones, mediante el burbujeo con aire usando una pipeta Pasteur, tiene lugar una rápida reacción que sucede en oscuridad donde se observa la desaparición del color rojo y, por consiguiente, de la banda de absorción antes mencionada. Este comportamiento se observa para Bip y Nep en ambas condiciones de pH. Además, es la misma conducta que describieron Pfleiderer et al. para la reacción de estos derivados pterínicos que tiene lugar en medio alcalino.3 Mediante evidencia empírica indirecta, los autores postulan que la banda de absorción en el visible correspondería al fotoproducto 6-formil-5,8-dihidropterina, compuesto que será analizado con mayor detalle en el siguiente capítulo. 


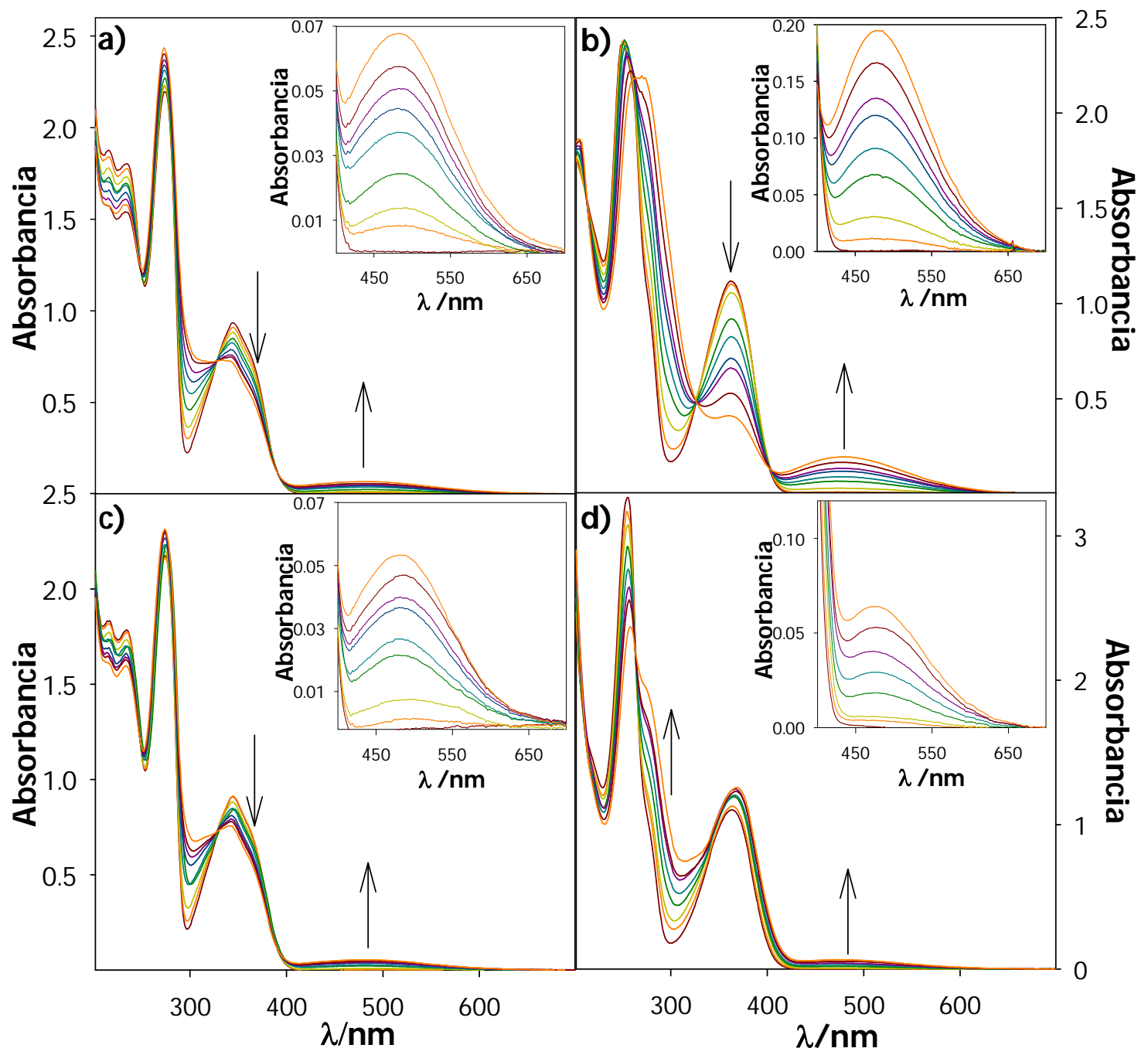

Figura 6.9 Evolución del espectro de absorción en función del tiempo de irradiación en ausencia de $\mathrm{O}_{2}$. a) $[\mathrm{Bip}]_{0}=142 \mu \mathrm{M}, \mathrm{pH}=5,0, \mathrm{ti}=0,15,30,45,60,75,90,105,120 \mathrm{~s}$; b) $\left[\mathrm{Bip}_{0}=158 \mu \mathrm{M}, \mathrm{pH}=10,6, \mathrm{ti}=0,15,30,45,60,75,90,120,180 \mathrm{~s} ; \mathrm{c}\right)[\mathrm{Nep}]_{0}=146 \mu \mathrm{M}, \mathrm{pH}$ $=5,1, \mathrm{ti}=0,15,30,45,60,75,90,105,120 \mathrm{~s}$; d) $[\mathrm{Nep}]_{0}=148 \mu \mathrm{M}, \mathrm{pH}=10,5, \mathrm{ti}=0,15,30$, 45, 60, 90, 105, $120 \mathrm{~s}$. Camino óptico $=1 \mathrm{~cm}$. Sistema de irradiación I.

Para determinar si el producto final de reacción era nuevamente Fop, se procedió a comparar los correspondientes EDEN y EDTN, como se describió anteriormente. En la Figura 6.10, se observan para Bip como los EDEN obtenidos de la resta del espectro de la solución sin irradiar al espectro de la solución irradiada (en ausencia de $\mathrm{O}_{2}$ ) e inmediatamente aireada, son comparables con los EDTN obtenidos restando el espectro de reactivo al de una solución estándar de Fop. Por lo tanto, Fop parece ser el producto final de la reacción luego de entrar $\mathrm{O}_{2}$ a la solución irradiada. Las pequeñas diferencias que se observan sugieren la formación de otros productos en menor proporción. Para Nep se observó el mismo comportamiento. 

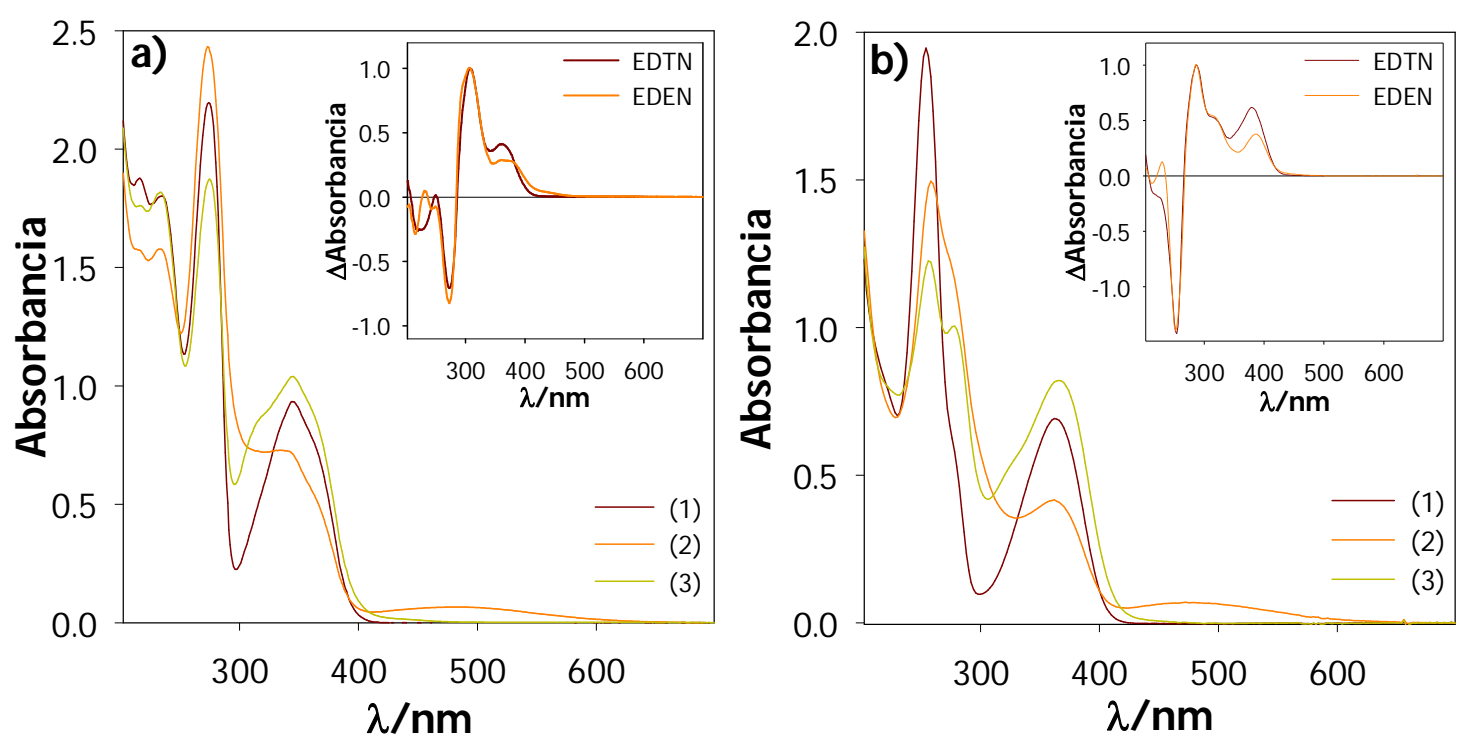

Figura 6.10 a) $[B i p]_{0}=150 \mu \mathrm{M}, \mathrm{pH}=5,5 ;$ b) $[B i p]_{0}=100 \mu \mathrm{M}, \mathrm{pH}=10,5$. Los números corresponden a (1) espectro de una solución no irradiada, (2) espectros de una solución irradiada 120 s en ausencia de $\mathrm{O}_{2}$, (3) espectro de la solución anterior aireada inmediatamente después de interrumpir la irradiación. Insets: EDEN obtenido de la diferencia entre el espectro (1) y el (3); y EDTN obtenido de la substracción de un espectro de Bip a un espectro de Fop en iguales condiciones de pH. Camino óptico $=1 \mathrm{~cm}$. Sistema de irradiación I.

Es importante mencionar que el estudio por HPLC de los productos de la fotólisis en condiciones anaeróbicas (previa a la entrada de aire en la celda) no fue posible, debido a que durante el proceso de inyección de las muestras en el cromatógrafo el contacto de los productos con el aire es inevitable. Por consiguiente, se analizaron las muestras irradiadas en ausencia de $\mathrm{O}_{2} \mathrm{y}$, posteriormente aireadas. El análisis por HPLC demostró que Fop es el producto principal de la fotólisis de Bip y Nep en el proceso global. Cabe aclarar que también se detectaron concentraciones muy bajas de Cap como fotoproducto. Esto puede deberse a la presencia de trazas de $\mathrm{O}_{2}$ no eliminado en el proceso de burbujeo previo a la irradiación. En los cromatogramas de la Figura 6.11, obtenidos para la fotólisis de Nep, se aprecia, además de los picos ya observados en las irradiaciones en aire, otros picos con áreas relativas muy pequeñas que corresponden a productos minoritarios. 

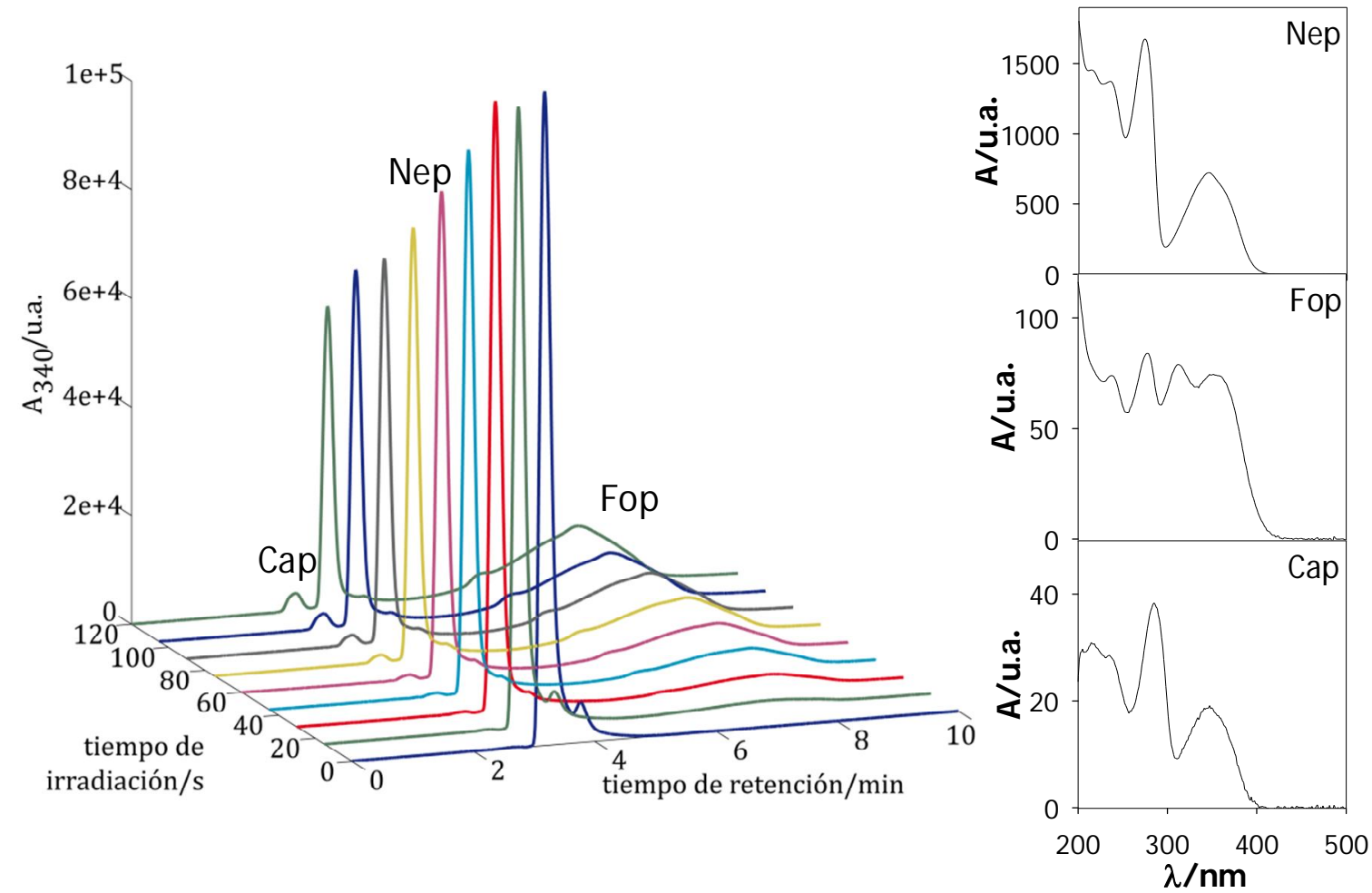

Figura 6.11 Cromatogramas $\left(\lambda_{\text {an }}=340 \mathrm{~nm}\right)$ obtenidos de la irradiación de una solución de Nep en ausencia de $\mathrm{O}_{2}\left([\mathrm{Nep}]_{0}=153 \mu \mathrm{M}, \mathrm{pH}=10,5\right)$. Espectros de absorción de Nep, Fop y Cap. Sistema de irradiación I y Equipo HPLC I.

Los perfiles de concentración se muestran en la Figura 6.12. Como puede observarse, la suma de concentraciones del reactivo y de los productos se mantiene casi constante a lo largo de todo el tiempo de irradiación. Esto confirma que el producto principal de reacción es nuevamente Fop y los productos mencionados en el párrafo anterior, se forman en proporciones muy pequeñas. Asimismo, se evaluó la generación de $\mathrm{H}_{2} \mathrm{O}_{2}$ una vez aireadas las soluciones irradiadas. En todos los casos, excepto para Nep en medio alcalino, la cantidad de $\mathrm{H}_{2} \mathrm{O}_{2}$ generado fue similar a la cantidad de reactivo consumido, dentro del error experimental (Figura 6.12). Curiosamente, para Nep en medio alcalino, la cantidad de $\mathrm{H}_{2} \mathrm{O}_{2}$ producido a cada tiempo de irradiación fue significativamente menor que la cantidad de reactivo consumido y también menor que la cantidad de Fop formada. Este comportamiento diferente fue confirmado en experimentos realizados partiendo de distintas concentraciones de Nep.

Es importante destacar que la velocidad de la fotólisis en ausencia de $\mathrm{O}_{2}$ es significativamente mayor que la observada para soluciones en presencia de dicho gas. A modo de ejemplo, en la Figura 6.13 se compara la irradiación de una solución de Bip en medio ácido, en presencia de aire con otra irradiada en ausencia de $\mathrm{O}_{2}$. Este 
comportamiento se observa para ambos compuestos, ya sea en medio ácido como en medio alcalino.
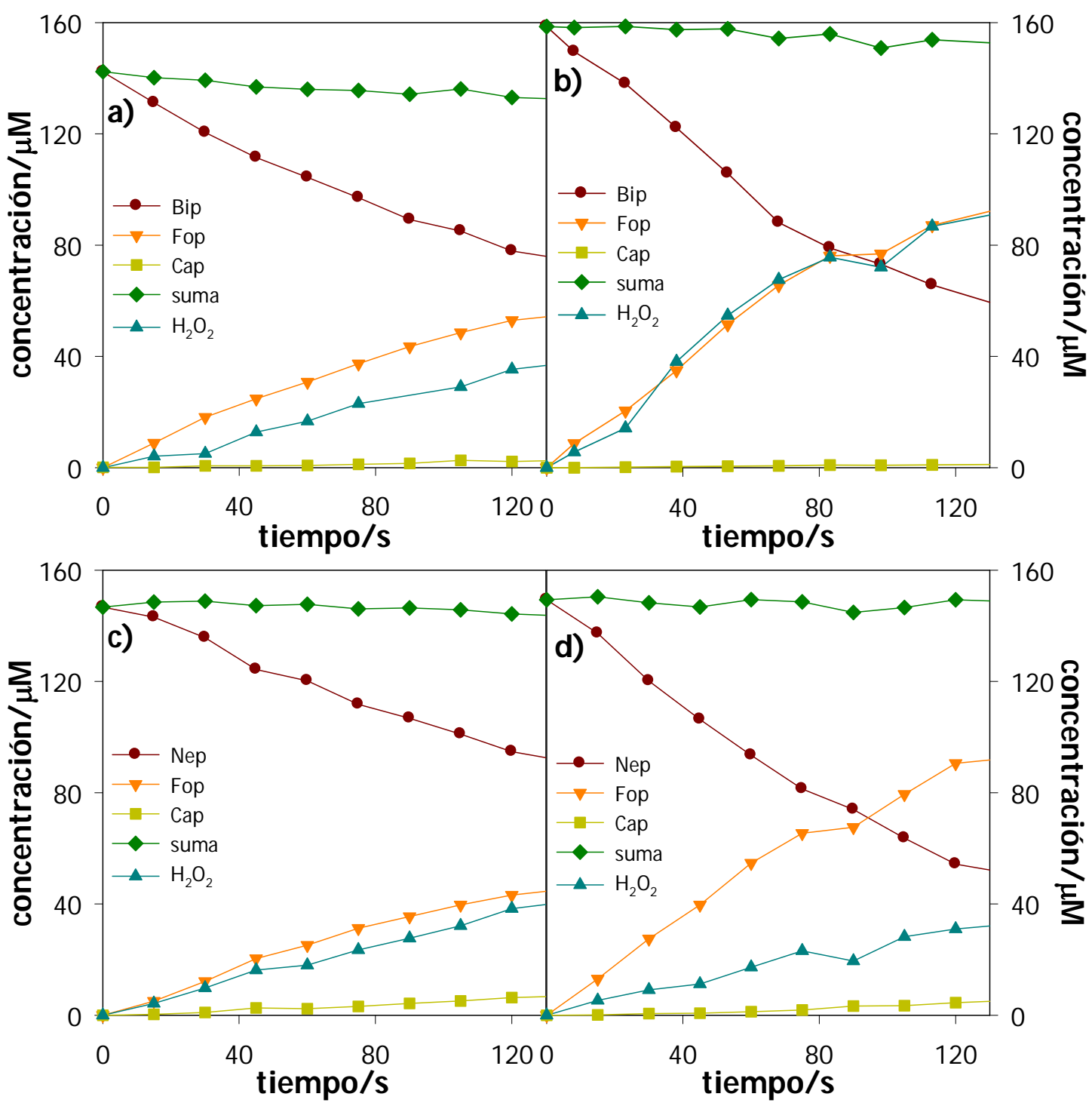

Figura 6.12 Perfiles de concentración de reactivos y productos para soluciones irradiadas en condiciones anaeróbicas y luego aireadas antes de la inyección en el cromatógrafo. a) $\left[\right.$ Bip $_{0}=142 \mu \mathrm{M}, \mathrm{pH}=5,0$; b) $\left.[\mathrm{Bip}]_{0}=158 \mu \mathrm{M}, \mathrm{pH}=10,6 ; \mathrm{c}\right)[\mathrm{Nep}]_{0}=146 \mu \mathrm{M}, \mathrm{pH}=5,1$; d) $[\mathrm{Nep}]_{0}=153 \mu \mathrm{M}, \mathrm{pH}=10,5$. Suma, corresponde a la suma de concentraciones de reactivo (Bip o Nep), Fop y Cap, a cada tiempo de irradiación. Sistema de irradiación I y Equipo HPLCI.

Como el $\mathrm{H}_{2} \mathrm{O}_{2}$ no puede ser generado durante la fotólisis en ausencia de $\mathrm{O}_{2}$, los resultados muestran que esta especie reactiva se forma en la reacción en oscuridad entre $\mathrm{O}_{2}$ y el intermediario rojo (IR). Además, es claro que en estas condiciones el oxígeno singlete $\left({ }^{1} \mathrm{O}_{2}\right)$ no puede participar como agente oxidante debido a que las soluciones se encuentran en anaerobiosis. Por lo tanto, es muy probable que el mecanismo de reacción sea el mismo para ambas condiciones estudiadas (presencia y ausencia de $\mathrm{O}_{2}$ ), con la única diferencia que en el último caso se observa el IR que, una 
vez que ingresa $\mathrm{O}_{2}$ en la celda de reacción, es capaz de reaccionar rápidamente con dicho gas, dando lugar a la consecuente oxidación.

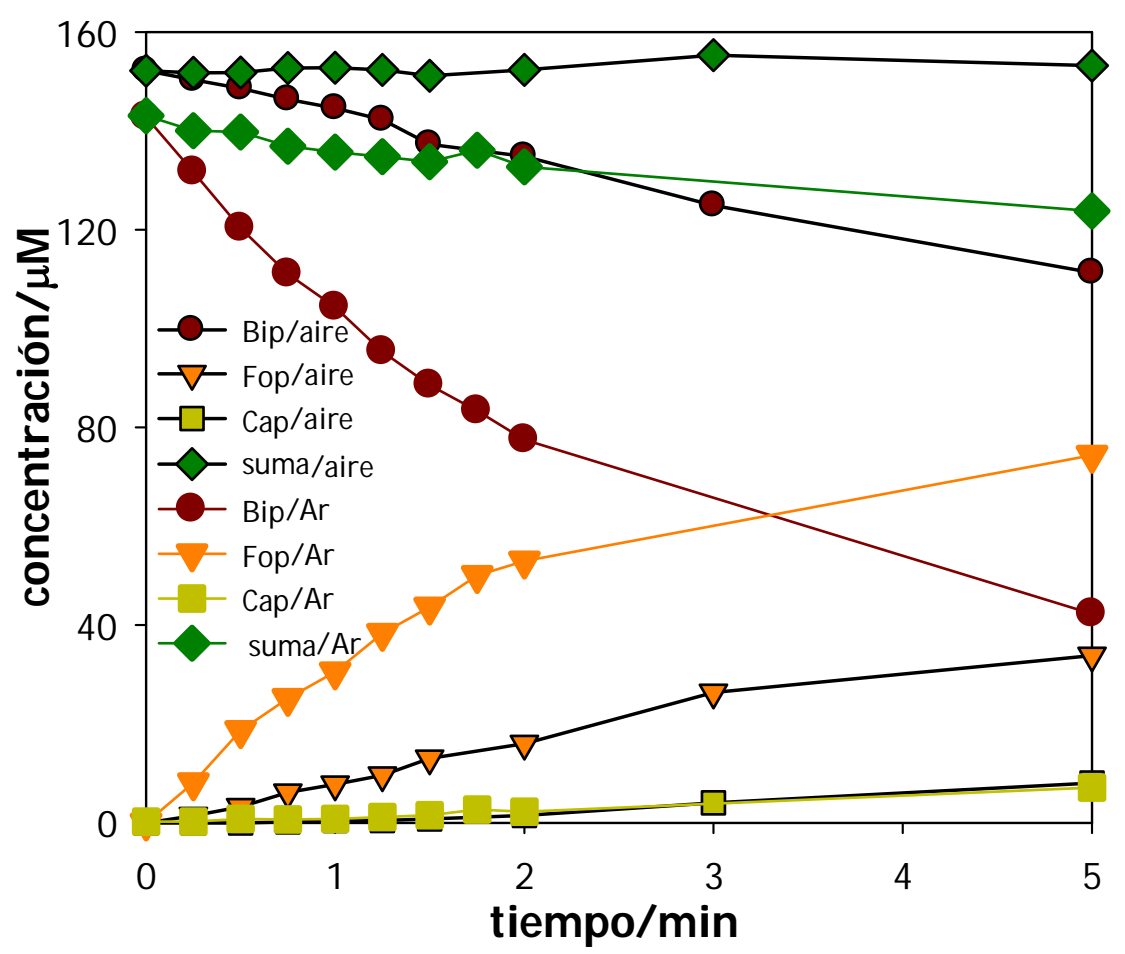

Figura 6.13 Perfiles de concentración de reactivos y productos para soluciones de Bip irradiadas en aire $\left([\mathrm{Bip}]_{0}=152 \mu \mathrm{M}, \mathrm{pH}=5,6\right)$ y en ausencia de $\mathrm{O}_{2}\left([\mathrm{Bip}]_{0}=143 \mu \mathrm{M}, \mathrm{pH}=\right.$ $5,0)$. Suma, corresponde a la suma de concentraciones de Bip, Fop y Cap, a cada tiempo de irradiación. Sistema de irradiación Iy Equipo HPLC I.

\subsection{Rendimientos cuánticos}

Se determinaron los rendimientos cuánticos de consumo de los reactivos ( $\Phi_{-\mathrm{R}}$, Bip y Nep) y de formación de productos $\left(\Phi_{\mathrm{P}}\right.$, Fop y $\left.\mathrm{H}_{2} \mathrm{O}_{2}\right)$ en medio ácido y alcalino, y a las distintas concentraciones de $\mathrm{O}_{2}$ ensayadas: ya sea en ausencia de $\mathrm{O}_{2}$, en aire y saturadas en $\mathrm{O}_{2}$. En el primer caso, las soluciones irradiadas fueron aireadas inmediatamente luego de finalizada la irradiación.

Para calcular los rendimientos cuánticos (Sección 4.4) es necesario tener en cuenta dos puntos. Primero, para determinar las velocidades iniciales $\left(-(d[R] / d t)_{0}\right.$ y $\left.(\mathrm{d}[\mathrm{P}] / \mathrm{dt})_{0}\right)$, los perfiles de concentración vs. tiempo de irradiación deben ser lineales. Generalmente, esta condición es real solo si la radiación absorbida por el reactivo $\left(\mathrm{q}_{\mathrm{p}, \lambda}\right)$ no disminuye significativamente $(<15 \%)$. Por lo tanto, para calcular $-(d[R] / d t)_{0}$ y $(\mathrm{d}[\mathrm{P}] / \mathrm{dt})_{0}$ en cada experimento se consideró solo el período de tiempo en que se cumplía esta condición. Para medio alcalino fue 60 s y $40 \mathrm{~s}$ para medio ácido. El otro punto a tener en cuenta es la fotólisis secundaria. Es decir, la reacción fotoquímica del producto, en este caso la fótolisis oxidativa de Fop a Cap, en los experimentos realizados 
en presencia de $\mathrm{O}_{2}$. Si esta reacción fuera significativa, en el intervalo de tiempo analizado, el valor calculado para (d[P]/dt) $)_{0}$ ) sería entonces incorrecto porque parte de Fop formada se habría consumido. Por lo tanto, en todos los experimentos, se prestó particular atención a la concentración de Cap, es decir el consumo de Fop, controlando que la misma fuera despreciable en el período de tiempo bajo análisis.

En la Tabla 6.2 se muestran los valores obtenidos para Bip y Nep bajo diferentes condiciones experimentales. En todos los casos, los rendimientos cuánticos de consumo de reactivo $\left(\Phi\right.$-sip y $\left.\Phi_{\text {-Nep }}\right)$ fueron iguales, dentro del error experimental, a los de formación de productos ( $\Phi_{\text {Fop }}$ ). Lo mismo se observó para la formación de $\mathrm{H}_{2} \mathrm{O}_{2}\left(\Phi_{\mathrm{H} 2 \mathrm{O} 2} \approx\right.$ $\left.\Phi_{-\mathrm{R}}\right)$ excepto para la fotólisis anaeróbica de Nep en medio alcalino. Esto indica que por cada molécula de reactivo consumido se genera una molécula de Fop y una de $\mathrm{H}_{2} \mathrm{O}_{2}$.

\begin{tabular}{|c|c|c|c|c|c|c|}
\hline & \multicolumn{3}{|c|}{$\mathrm{pH}=5,5$} & \multicolumn{3}{|c|}{$\mathrm{pH}=10,5$} \\
\hline & $\mathrm{Ar}$ & Aire & $\mathrm{O}_{2}$ & $\mathrm{Ar}$ & Aire & $\mathrm{O}_{2}$ \\
\hline \multicolumn{7}{|c|}{ Fotólisis de Bip } \\
\hline$\Phi$-Bip & $0,10 \pm 0,01$ & $0,037 \pm 0,003$ & $0,024 \pm 0,003$ & $0,18 \pm 0,02$ & $0,12 \pm 0,01$ & $0,07 \pm 0,01$ \\
\hline$\Phi_{\mathrm{Fop}}$ & $0,10 \pm 0,01$ & $0,037 \pm 0,003$ & $0,024 \pm 0,003$ & $0,18 \pm 0,02$ & $0,12 \pm 0,01$ & $0,07 \pm 0,01$ \\
\hline$\Phi_{\text {Н202 }}$ & $0,07 \pm 0,01$ & $0,037 \pm 0,005$ & $0,031 \pm 0,005$ & $0,15 \pm 0,05$ & $0,13 \pm 0,02$ & $0,08 \pm 0,01$ \\
\hline
\end{tabular}

Fotólisis de Nep

$\begin{array}{ccccccc}\Phi_{\text {-Nep }} & 0,11 \pm 0,01 & 0,044 \pm 0,003 & 0,018 \pm 0,002 & 0,16 \pm 0,03 & 0,11 \pm 0,01 & 0,069 \pm 0,005 \\ \Phi_{\text {Fop }} & 0,10 \pm 0,01 & 0,043 \pm 0,004 & 0,019 \pm 0,002 & 0,16 \pm 0,03 & 0,10 \pm 0,01 & 0,070 \pm 0,005 \\ \Phi_{\text {H202 }} & 0,08 \pm 0,01 & 0,05 \pm 0,01 & 0,023 \pm 0,004 & 0,06 \pm 0,01 & 0,09 \pm 0,02 & 0,07 \pm 0,01\end{array}$

Tabla 6.2 Rendimientos cuánticos de consumo de reactivo ( $\Phi$.r) y generación de producto $\left(\Phi_{\mathrm{P}}\right)$ para las fotólisis de Bip y Nep en distintas condiciones.

De la Tabla 6.2 se deduce que, a una condición de pH y concentración de $\mathrm{O}_{2}$ determinada, Bip y Nep tienen $\Phi_{-\mathrm{R}}$ muy similares entre sí. Además, si se comparan las dos condiciones de $\mathrm{pH}$, ambas pterinas tienen menor $\Phi_{-\mathrm{R}}$ en medio ácido que en medio alcalino. Asimismo, para ambos reactivos y en las dos condiciones de $\mathrm{pH}$, los $\Phi \cdot \mathrm{R}$ dependen de la concentración de $\mathrm{O}_{2}$ : a mayor concentración de $\mathrm{O}_{2}$, menor es el rendimiento cuántico. Este resultado sugiere que el comportamiento general de ambas pterinas es el mismo y que el estado excitado del reactivo involucrado en la reacción fotoquímica es desactivado por el $\mathrm{O}_{2}$ disuelto. Este punto será discutido con mayor detalle en la siguiente sección. 


\subsection{Análisis de los estados excitados}

De acuerdo a lo descripto previamente, en las tres condiciones de concentración de $\mathrm{O}_{2}$ utilizadas, se observa que las reacciones globales son las mismas. Sin embargo los rendimientos cuánticos dependen fuertemente de la concentración de $\mathrm{O}_{2}$. Esto sugiere, como se mencionó anteriormente, que el $\mathrm{O}_{2}$ desactiva eficientemente el estado excitado del reactivo a partir del cual se generan los fotoproductos.

Para investigar la desactivación del estado excitado singlete de Bip y Nep por $\mathrm{O}_{2}$ se realizaron experimentos de emisión de fluorescencia (Sección 5.2). Se registraron los espectros de emisión de soluciones ácidas y alcalinas de ambas pterinas, bajo diferentes concentraciones de $\mathrm{O}_{2}$ ( $\mathrm{Ar}$, aire y en condiciones de saturación de $\mathrm{O}_{2}$ ). En la Figura 6.14 se aprecian los espectros de emisión para soluciones de Bip en medio ácido y alcalino. Se encontró que la emisión de fluorescencia es independiente de la concentración de $\mathrm{O}_{2}$ en la solución. Estos resultados indican que el estado excitado singlete del reactivo no es desactivado por $\mathrm{O}_{2} \mathrm{y}$, por consiguiente, no participa en el proceso fotoquímico. Para Nep se obtuvieron resultados muy similares.
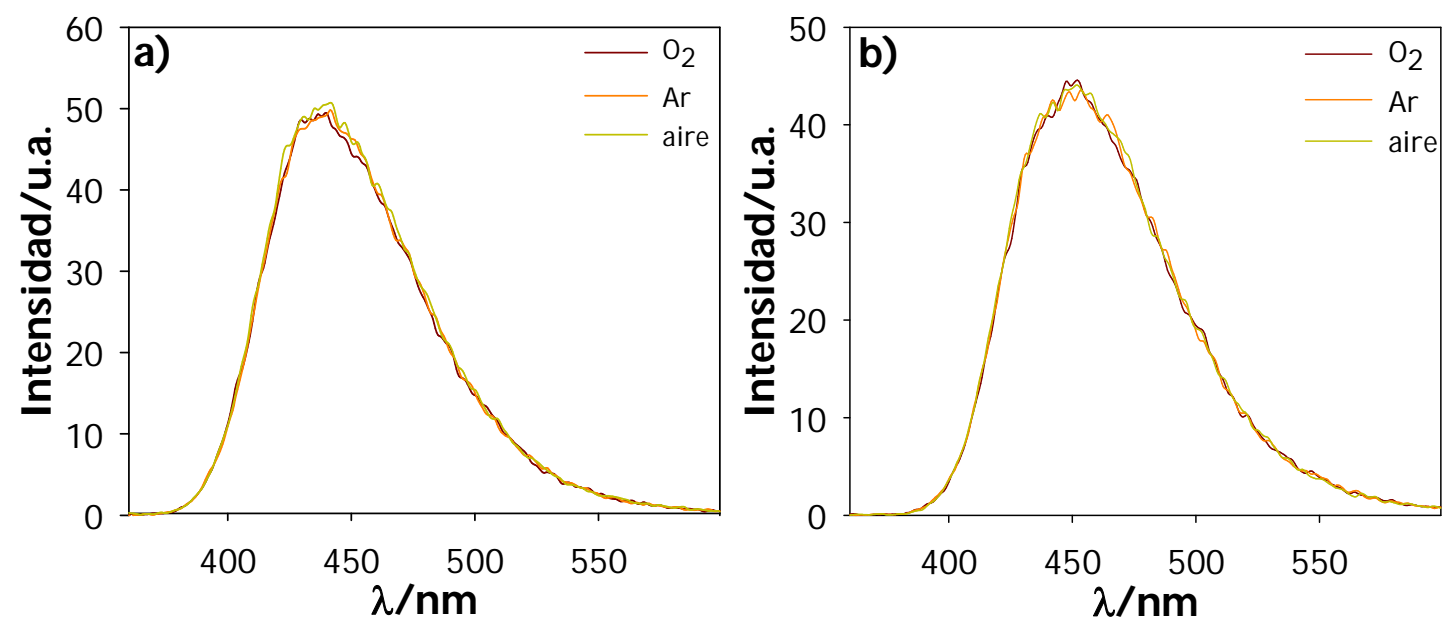

Figura 6.14 Espectros de fluorescencia de Bip en soluciones con distintas concentraciones de $\mathrm{O}_{2}$. a) $\left.\mathrm{pH}=5,6 ; \mathrm{b}\right) \mathrm{pH}=10,5$.

Consecuentemente, se propone que el estado excitado involucrado en la reacción fotoquímica es el triplete. Esto es esperable teniendo en cuenta que Bip, Nep y otros derivados pterínicos producen eficientemente estados excitados tripletes, ${ }^{7,8}$ que son desactivados por $\mathrm{O}_{2}$ para generar ${ }^{1} \mathrm{O}_{2} .{ }^{9}$ Por ejemplo, para Bip se conocen los valores de constante de velocidad de desactivación del estado excitado triplete por $\mathrm{O}_{2}\left(\mathrm{k}_{\mathrm{et}}\right)$, siendo de $1,4 \times 10^{9} \mathrm{M}^{-1} \mathrm{~s}^{-1}$ y 3,2 x $10^{9} \mathrm{M}^{-1} \mathrm{~s}^{-1}$ en medio ácido y alcalino, respectivamente. ${ }^{8}$ De esta manera, se puede describir un mecanismo de reacción parcial donde el reactivo absorbe 
energía para pasar al estado excitado singlete, que luego por entrecruzamiento de sistemas pasa al estado excitado triplete, el cual genera finalmente el IR. Este mecanismo se resume en el Esquema 6.2.

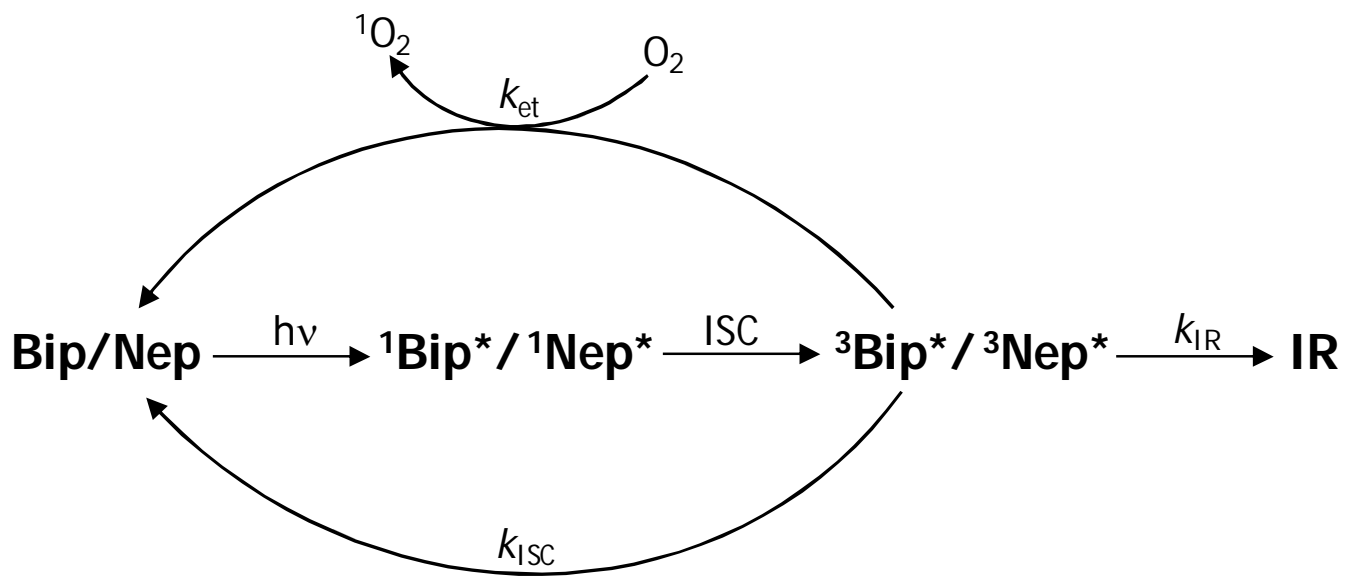

Esquema 6.2 Mecanismo parcial con intervención de estados excitados.

Si este mecanismo fuese correcto, planteando la hipótesis de estado estacionario para el estado excitado triplete de las pterinas estudiadas, $\Phi_{-\mathrm{R}}$ vendría dado por la siguiente ecuación:

$$
\Phi_{-\mathrm{R}}=\Phi_{\mathrm{T}} \frac{\mathrm{k}_{\mathrm{IR}}}{\mathrm{k}_{\mathrm{IR}}+\mathrm{k}_{\mathrm{ISC}}+\mathrm{k}_{\mathrm{et}}\left[\mathrm{O}_{2}\right]}
$$

donde $\Phi_{\mathrm{T}}$ es el rendimiento cuántico de formación del estado excitado triplete; $\mathrm{k}_{\mathrm{IR}}$ es la constante de velocidad de reacción de formación del IR $\left(\mathrm{s}^{-1}\right)$; $\mathrm{k}_{\mathrm{ISC}}$ es la constante de velocidad de desactivación por entrecruzamiento de sistemas $\left(\mathrm{s}^{-1}\right) ; \mathrm{k}_{\text {et }}$ es la constante de velocidad de desactivación del estado excitado triplete por $\mathrm{O}_{2}\left(\mathrm{M}^{-1} \mathrm{~S}^{-1}\right)$.

La relación $\Phi_{-\mathrm{A}}^{\mathrm{Ar}} / \Phi \cdot \mathrm{R}$ está dada por:

$$
\frac{\Phi_{-\mathrm{R}}^{\mathrm{Ar}}}{\Phi_{-\mathrm{R}}}=\frac{\mathrm{k}_{\mathrm{IR}}+\mathrm{k}_{\mathrm{ISC}}+\mathrm{k}_{\mathrm{et}}\left[\mathrm{O}_{2}\right]}{\mathrm{k}_{\mathrm{IR}}+\mathrm{k}_{\mathrm{ISC}}}=1+\mathrm{k}_{\mathrm{et}} \tau_{\mathrm{T}}\left[\mathrm{O}_{2}\right]=1+\mathrm{k}_{\mathrm{SV}}\left[\mathrm{O}_{2}\right]
$$

Entonces, realizando una gráfica de Stern-Volmer de $\Phi_{-\mathrm{R}} \mathrm{Ar} / \Phi_{-\mathrm{R}}$ en función de la concentración de $\mathrm{O}_{2}$ (Figura 6.15) y a partir de la ecuación anterior, donde $\mathrm{ksv}_{\mathrm{sv}}=\mathrm{k}_{\mathrm{et}} \tau_{\mathrm{T}}, \mathrm{y}$ $\tau_{\mathrm{T}}$ es alrededor de $2 \mu$ s (Capítulo 3), se puede calcular ket. Para ambas pterinas en medio ácido y alcalino, se obtienen valores de entre 0,5 y $2,0 \times 10^{9} \mathrm{M}^{-1} \mathrm{~s}^{-1}$. Estos valores son del orden de las constantes publicadas por Ledbetter et al., lo cual sugiere nuevamente que el estado excitado triplete estaría involucrado en la reacción de formación del IR. 


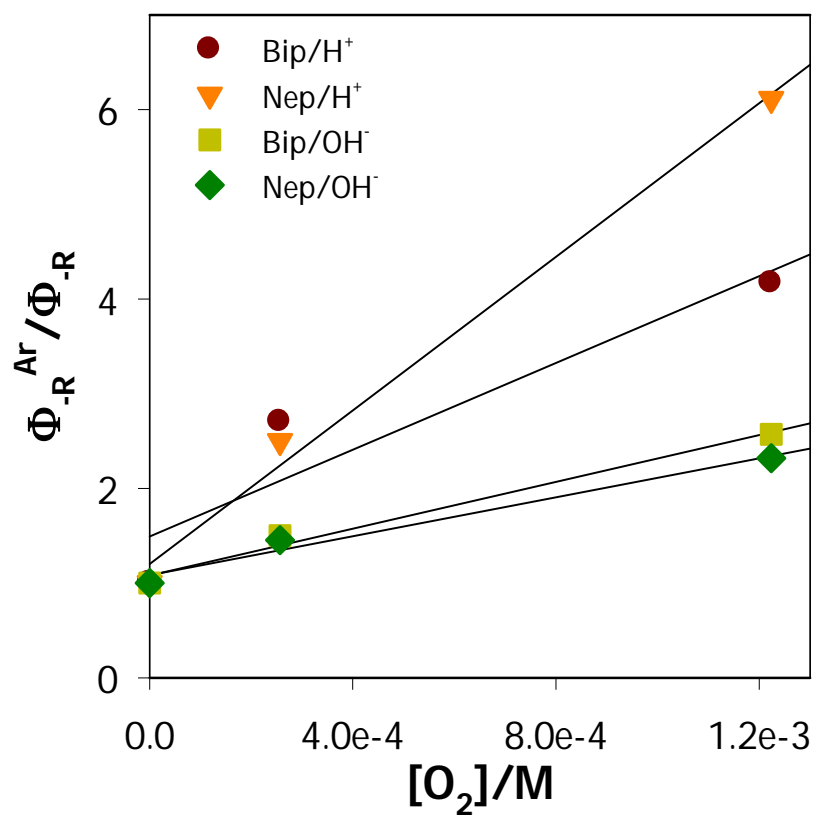

Figura 6.15 Gráfica de Stern-Volmer: variación de $\Phi_{-\mathrm{R}} \mathrm{Ar} / \Phi_{-\mathrm{R}}$ con la concentración de $\mathrm{O}_{2}$.

\subsection{Investigación de anión superóxido}

Dado que $\mathrm{H}_{2} \mathrm{O}_{2}$ puede generarse a partir del anión superóxido $\left(\mathrm{O}_{2}{ }^{--}\right),{ }^{10}$ se decidió investigar la existencia de esta especie reactiva al irradiar soluciones de Bip y Nep. Para esto, se utilizaron dos métodos de detección: (i) método reducción de citocromo c (Cyt) inhibible por superóxido dismutasa (SOD) (Sección 5.9) y (ii) resonancia paramagnética electrónica (Sección 5.6).

\subsubsection{Método de Cyt}

Soluciones equilibradas en aire de Bip o Nep $(75 \mu \mathrm{M})$ se mezclaron con una solución de Cyt (14 $\mu \mathrm{M})$, en medio ácido o alcalino. Dichas mezclas fueron irradiadas con el Sistema de irradiación I, registrando los espectros de absorción cada 10 s durante un tiempo de irradiación total de $2 \mathrm{~min}$. En la Figura 6.16.a se muestran los espectros de absorción en función del tiempo de irradiación, donde claramente se aprecia el incremento de la absorbancia en el rango 500 - $600 \mathrm{~nm}$, consistente con la reducción de Cyt. Los EDEN en ese rango de longitudes de onda, detallados en la Figura 6.16.b, son similares a los reportados en literatura para la reducción de Cyt. ${ }^{11}$ En la Figura 6.17 se muestra el aumento de absorbancia a $550 \mathrm{~nm}\left(\mathrm{~A}^{550}\right)$, en función del tiempo de irradiación $(--)$, para la solución mezcla antes mencionada. Para confirmar que la reducción de Cyt es debido al $\mathrm{O}_{2}{ }^{*}$, se realizaron experimentos similares agregando SOD $(200 \mathrm{U} / \mathrm{ml})(\bullet-)$, enzima que cataliza la reacción de dismutación de $\mathrm{O}_{2}{ }^{--}$para dar $\mathrm{O}_{2} \mathrm{y}$ 
$\mathrm{H}_{2} \mathrm{O}_{2}$. Se observó una clara inhibición de la fotorreducción de Cyt, sugiriendo que bajo las condiciones experimentales empleadas, el $\mathrm{O}_{2}{ }^{*}$ generado fotoquímicamente por Bip o Nep le transfiere un electrón a Cyt para reducirlo de ferricitocromo c $\left(\mathrm{Fe}^{+3}\right)$ a ferrocitocromo c $\left(\mathrm{Fe}^{+2}\right)$.

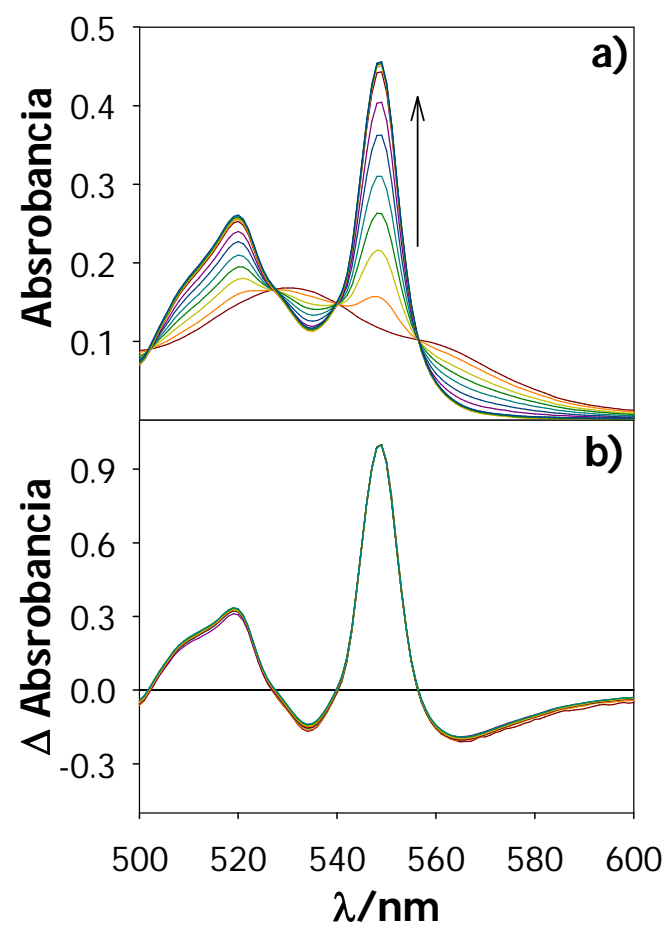

Figura 6.16 a) Espectros de absorción de una solución mezcla en presencia de $\mathrm{O}_{2}\left([\mathrm{Bip}]_{0}=\right.$ $75 \mu \mathrm{M}$, [Cyt] $=14 \mu \mathrm{M}, \mathrm{pH}=5,6$ ) registrados cada $10 \mathrm{~s}$, hasta 2 min totales de irradiación; b) EDEN. Camino óptico $=1 \mathrm{~cm}$. Sistema de irradiación $\mathrm{I}$.

Cuando se realizaron los mismos experimentos pero en ausencia de $\mathrm{O}_{2}$, es decir, en condiciones donde no tiene lugar la formación de $\mathrm{O}_{2}{ }^{*}$, la fotorreducción de Cyt también fue observada $(-\bullet)$, incluso en presencia de SOD $(\rightarrow-)$. Más aún, el grado de reducción de Cyt fue mayor que en presencia de aire, alcanzando un plateau a menores tiempos de irradiación. Esto sugiere que el proceso que ocurre en condiciones anaeróbicas es una transferencia de electrones directa desde el compuesto pterínico al Cyt. Esto ya ha sido observado para otros compuestos heterociclos en solución acuosa.12, 13

Para descartar la existencia de procesos térmicos o fotoquímicos que puedan interferir en el ensayo, se realizaron diversos experimentos de control. En primer lugar, no se observó reducción de Cyt en ninguno de los dos medios, cuando se mantuvieron en oscuridad soluciones de Bip o Nep (75 $\mu \mathrm{M})$ y Cyt (14 $\mu \mathrm{M})$ por más de 10 min. Asimismo, se irradiaron soluciones conteniendo sólo Cyt y tampoco se observaron cambios espectrales. 

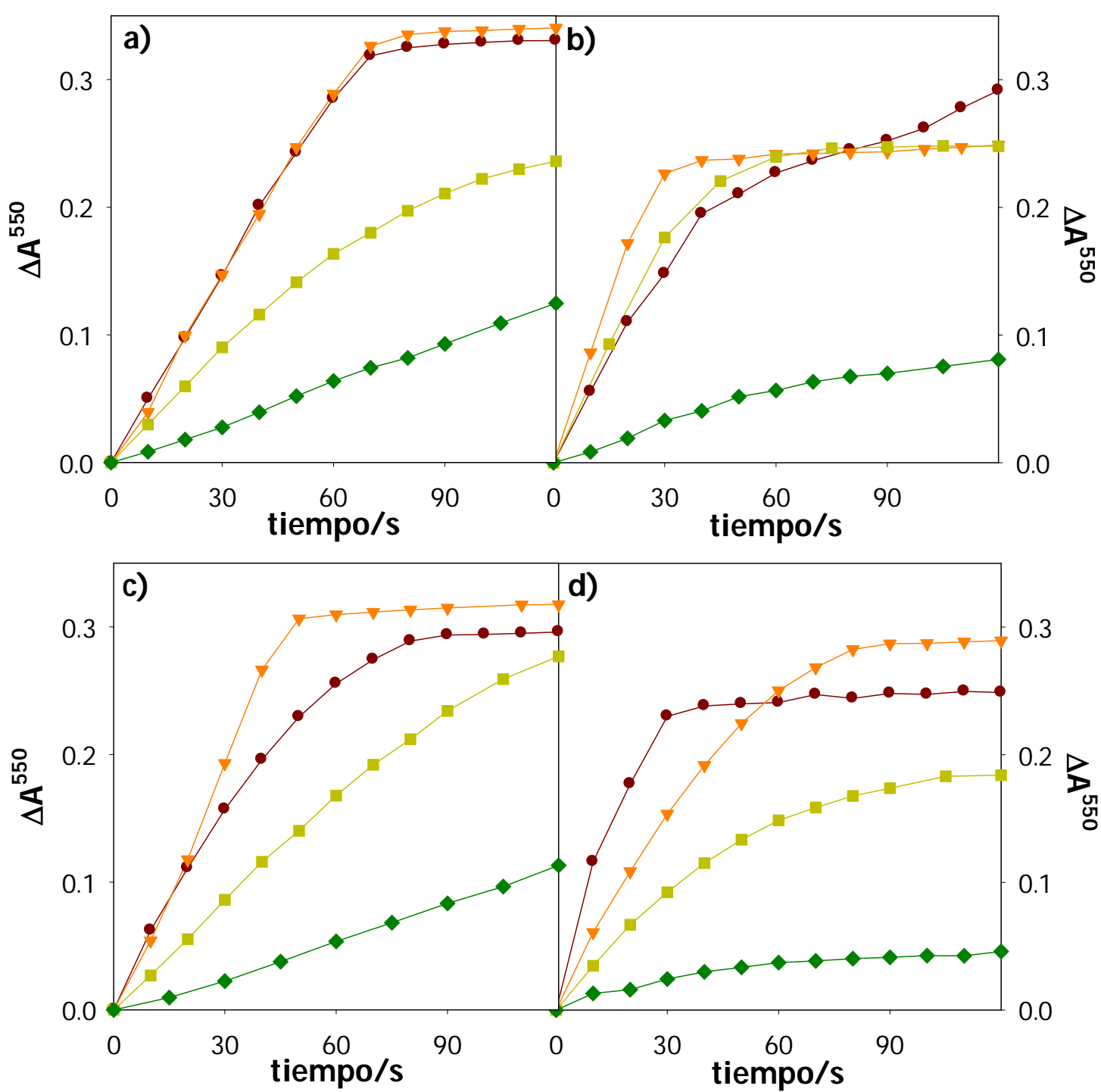

Figura 6.17 Evolución de $\Delta \mathrm{A}^{550}$ de solución mezcla de Bip o Nep (75 $\left.\mu \mathrm{M}\right)$ y Cyt (14 $\left.\mu \mathrm{M}\right)$ con el tiempo de irradiación, en presencia (aire) y ausencia de $\mathbf{O}_{2}$ (Ar). $\rightarrow \mathrm{Pt}+$ Cyt (Ar), $\rightarrow \mathrm{Pt}+\mathrm{Cyt}+\mathrm{SOD}(\mathrm{Ar}),-\mathrm{Pt}+\mathrm{Cyt}$ (aire), $\rightarrow \mathrm{Pt}+\mathrm{Cyt}+\mathrm{SOD}$ (aire). a) Bip, $\mathrm{pH}=5,1$; b) Bip, $\mathrm{pH}=10,4$; c) Nep, $\mathrm{pH}=5,0$; d) Nep, $\mathrm{pH}=10,3$. Sistema de irradiación I.

Consecuentemente, teniendo en cuenta los estudios previos reportados en literatura, sobre la fotooxidación de distintos derivados pterínicos en combinación con los resultados descriptos en este capítulo, se puede concluir que el intermediario generado en la irradiación de Bip y Nep es capaz de transferir un electrón al $\mathrm{O}_{2}$ para generar $\mathrm{O}_{2}{ }^{-} \mathrm{o}$, directamente, al Cyt para reducirlo. En soluciones aireadas, donde hay exceso de $\mathrm{O}_{2}$ con respecto a Cyt, el primer proceso tiene lugar. Sin embargo, en ausencia de $\mathrm{O}_{2}$, el segundo proceso será el que predomine. Las reacciones que ocurren en presencia y ausencia de $\mathrm{O}_{2}$ se detallan en el Esquema 6.3. 


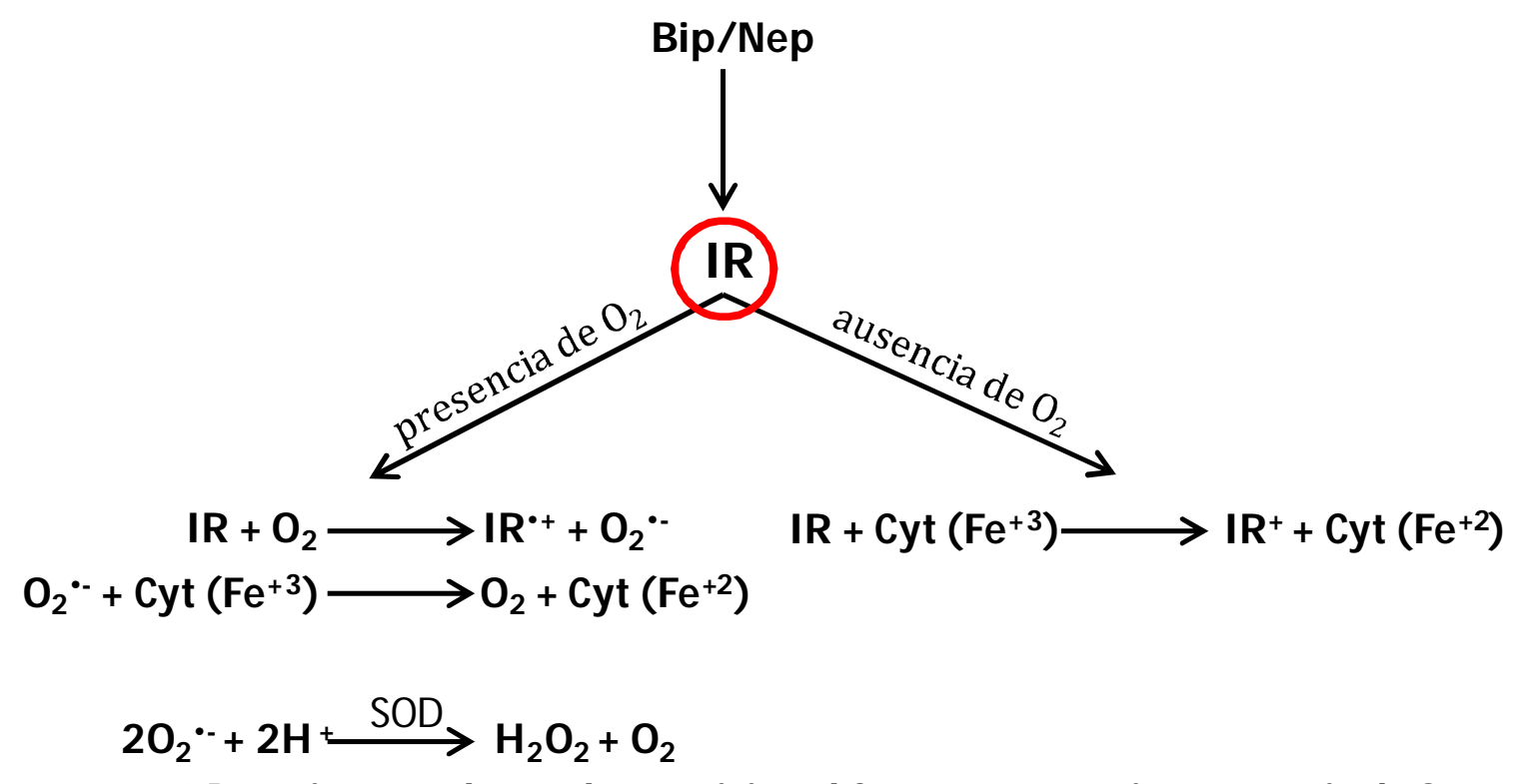

Esquema 6.3 Reacciones en las cuales participa el Cyt c en presencia o ausencia de $\mathrm{O}_{2}$.

\subsubsection{Resonancia Paramagnética Electrónica}

El método de Cyt inhibible por SOD, aunque es muy utilizado, no es confirmatorio de la generación de $\mathrm{O}_{2}{ }^{-}$, motivo por el cual se recurrió a la técnica Resonancia Paramagnética Electrónica (RPE). Esta técnica, explicada en la Sección 5.6, permite detectar diferentes compuestos radicales. En el caso de $\mathrm{O}_{2}{ }^{*}$, si bien por su carácter paramagnético podría ser detectado directamente, el hecho de tener un tiempo de vida media muy corto $\left(\sim 10^{-5} \mathrm{~s}\right)$ hace muy difícil esta tarea. Por lo tanto, se recurrió al método de secuestro selectivo utilizando 5,5-dimetil-1-pirrolin-N-óxido (DMPO). ${ }^{14}$ Este compuesto forma un aducto con $\mathrm{O}_{2}{ }^{*-}$ de mayor vida media y, por lo tanto, resulta más viable su detección por RPE.

Se mezclaron soluciones de Bip o Nep $(100 \mu \mathrm{M})$ con DMPO $(50 \mathrm{mM})$ y buffer Tris/ $\mathrm{HCl}(1 \mathrm{mM})-\mathrm{NaCl}(0,5 \mathrm{mM})(\mathrm{pH}=7,2)$. Las mezclas se introdujeron en celdas de cuarzo de paredes planas y se irradiaron directamente dentro de la cavidad del espectrómetro, tal como se explica en la Sección 5.6. Se irradió por $40 \mathrm{~s}$, se tomó el espectro donde se observó la señal característica del aducto DMPO-OOH. Para confirmar la formación de $\mathrm{O}_{2} \cdot$ - durante la irradiación de Bip y Nep se realizaron experimentos similares en presencia de SOD (22 U/ ml). Como se aprecia en la Figura 6.18, la señal del aducto disminuye significativamente. Además, se realizaron experimentos control irradiando solo DMPO, o registrando el espectro a la mezcla PtDMPO pero sin irradiar y en ninguno de los caso se observó la señal del aducto. 


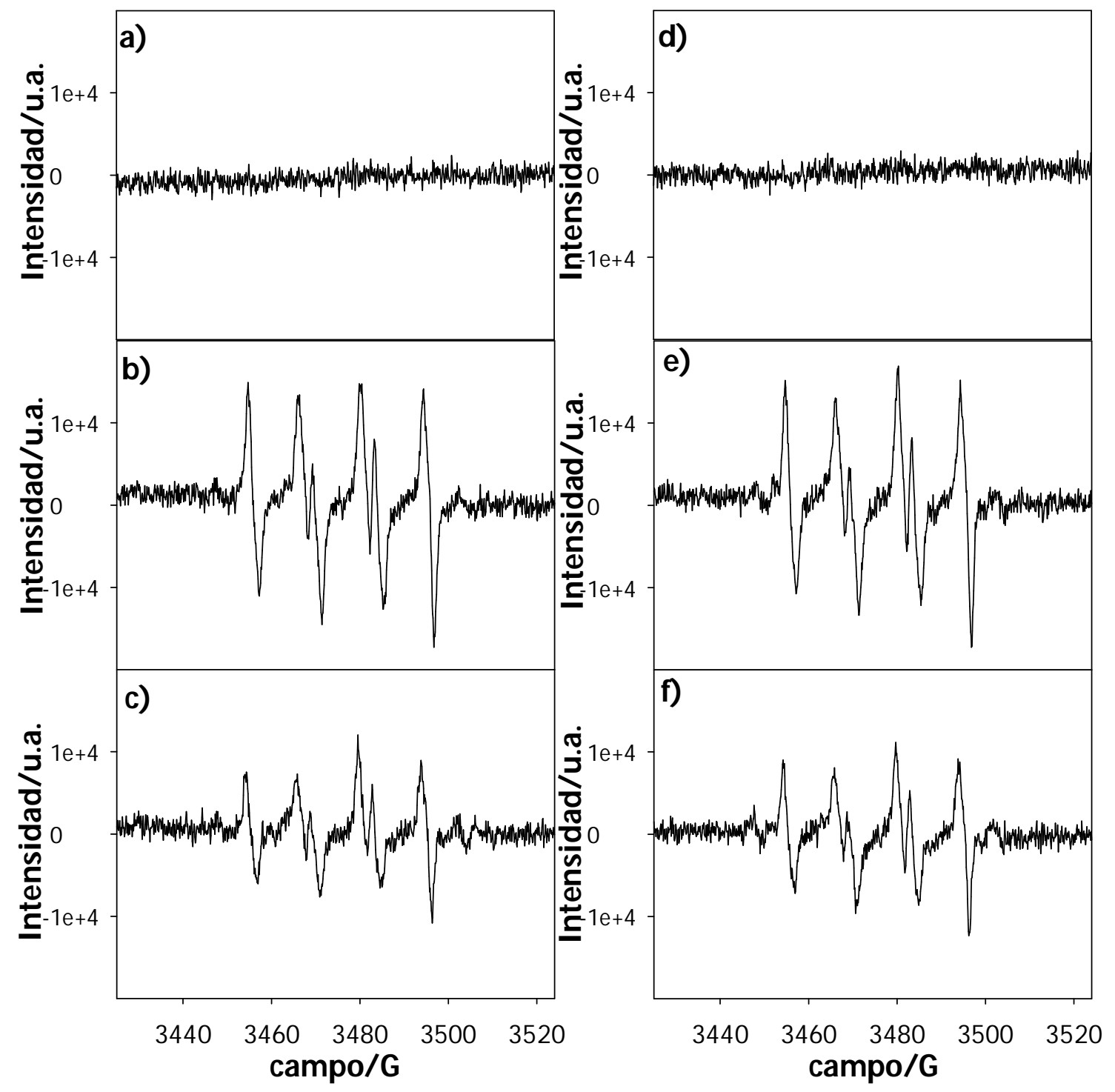

Figura 6.18 Detección del aducto DMPO•-OOH por RPE. Solución mezcla de Pt (100 $\mu \mathrm{M})$ y DMPO (50 mM) a pH = 7,0. a) Bip; b) Bip, ti = $40 \mathrm{~s}$; c) Bip + SOD (22 U/ ml), ti = 40 s; d) Nep; e) Nep, ti = $40 \mathrm{~s}$; f) Nep + SOD (22 U/ ml), ti = $40 \mathrm{~s}$.

Es importante destacar que ciertas pterinas, como Ptr y Mep, generan $\mathrm{O}_{2}{ }^{-}-$por un mecanismo completamente diferente al mencionado en este capítulo. Es decir, cuando Ptr es excitada y, por entrecruzamiento de sistemas pasa al estado excitado triplete (3Ptr*), puede reaccionar con otra molécula de Ptr u otro dador de electrones, generando el anión radical $\operatorname{Ptr}\left(\mathrm{Ptr}^{*-}\right)$. Este, a su vez, reacciona con $\mathrm{O}_{2}$ para formar $\mathrm{O}_{2}{ }^{\circ-}$, regenerando así Ptr. ${ }^{15}$ Este mecanismo es completamente diferente al que sucede en el caso de Bip y Nep donde $\mathrm{O}_{2}{ }^{*-}$ es generado a partir del IR, tal como se explicó previamente y se detalla en las reacciones siguientes: 


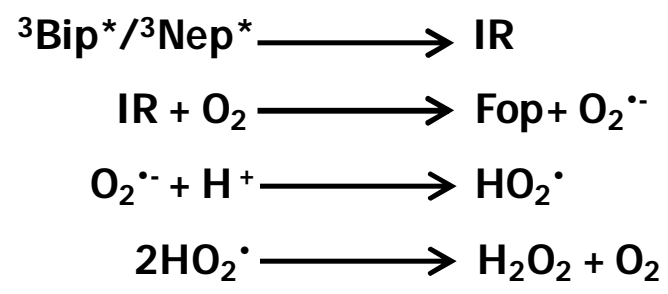

\subsection{Conclusiones}

En este capítulo se estudiaron diversos aspectos de la fotoquímica de Bip y Nep en solución acuosa cuyo esclarecimiento aún quedaban pendientes. Cuando Bip o Nep son expuestas a la radiación UV-A, un intermediario rojo (IR) es formado en un proceso independiente del $\mathrm{O}_{2}$. Luego, este producto es rápidamente oxidado por $\mathrm{O}_{2}$ para generar Fop y $\mathrm{H}_{2} \mathrm{O}_{2}$. Este mecanismo es válido para las formas ácidas y básicas de Bip y Nep. Además, la cuantificación de los reactivos y productos reflejan que al realizar la fotólisis en ausencia de $\mathrm{O}_{2}$ no existen vías adicionales de consumo de reactivo.

Otra especie reactiva de oxígeno, el anión superóxido $\left(\mathrm{O}_{2}{ }^{*}\right)$, es también generada durante la fotooxidación de Bip y Nep. Esto tiene lugar a través de un proceso de transferencia de electrones desde el intermediario rojo al $\mathrm{O}_{2}$ disuelto. Este hecho es muy importante desde el punto de vista biomédico porque este radical está implicado en la etiología de varias patologías. Además, considerando que Bip se acumula en las manchas de pacientes con vitíligo, estos resultados sugieren que la producción fotoinducida de EROs por Bip podría contribuir al estrés oxidativo involucrado en la patogénesis de esta enfermedad.

Para ambos reactivos y a cada condición de $\mathrm{pH}$, el rendimiento cuántico de consumo del reactivo depende de la concentración de $\mathrm{O}_{2}$ : a mayor concentración de $\mathrm{O}_{2}$, menor es el rendimiento cuántico. Este comportamiento puede explicarse en base la desactivación del estado excitado triplete del reactivo por parte del $\mathrm{O}_{2}$ disuelto. Teniendo en cuenta los resultados de los experimentos de fluorescencia a diferentes concentraciones de $\mathrm{O}_{2}$ y trabajos previos, se puede proponer que los estados electrónicos excitados que participan de la reacción son de naturaleza triplete.

Por lo tanto, se puede proponer un mecanismo general para las dos pterinas estudiadas, como se detalla en el Esquema 6.4. 


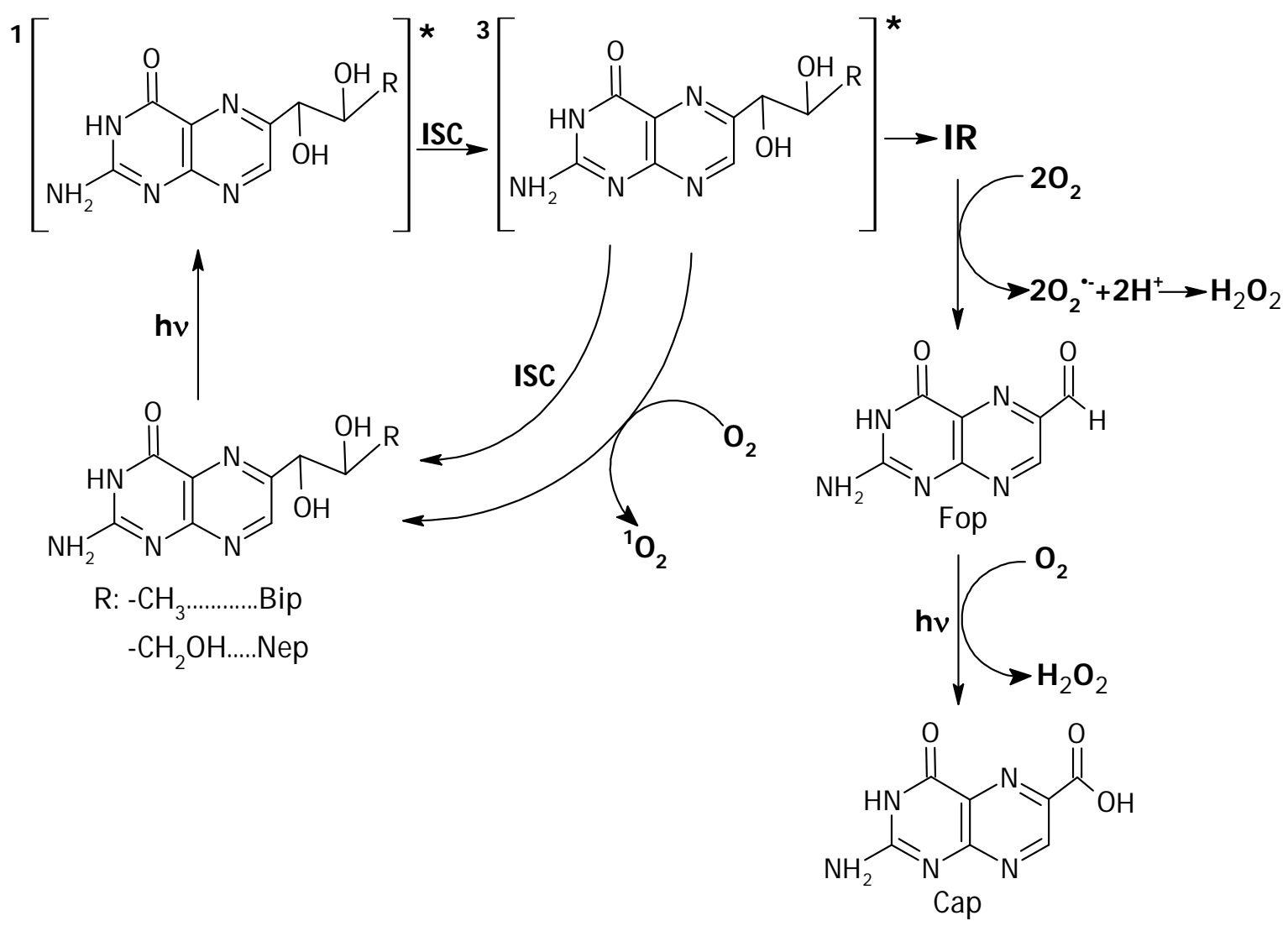

Esquema 6.4 Mecanismo general de fotooxidación para Bip y Nep

\subsection{Referencias}

1. H. S. Forrest and H. K. Mitchell, Pteridines from Drosophila. III. Isolation and Identification of Three More Pteridines, J. Am. Chem. Soc., 1955, 77, 4865-4869.

2. M. Viscontini and H. Raschig, Fluoreszierende Stoffe aus Drosophila melanogaster. 7th Mitteilung, Helv. Chim. Acta, 1958, 41, 108-113.

3. R. Mengel, W. Pfleiderer and W. R. Knappe, Norrish type II analogous photodegradation of neopterin and biopterin to 5,8 -dihydropterin-6-aldehyde, Tetrahedron Lett., 1977, 18, 2817-2820.

4. R. Baur, M. Kappel, R. Mengel and W. Pfleiderer, Photochemistry of pteridines, in Chemistry and Biology of Pteridines, ed. R. L. Kisliuk and G. M. Brown, Elsevier, New York, 1979, pp. 13-18.

5. A. H. Thomas, G. Suárez, F. M. Cabrerizo and A. L. Capparelli, Photochemistry of 6-formylpterin in alkaline medium, Helvetica Chimica Acta, 2001, 84, 3849-3860.

6. G. Petroselli, J. M. Bartsch and A. H. Thomas, Photoinduced generation of $\mathrm{H}_{2} \mathrm{O}_{2}$ and $\mathrm{O}_{2} *$ by 6formylpterin in aqueous solutions, Pteridines, 2006, 17, 82-89.

7. C. Chahidi, M. Aubailly, A. Momzikoff, M. Bazin and R. Santus, Photophysical and photosensitizing propierties of 2-amino-4 pteridinone: a natural pigment, Photochemistry and Photobiology, 1981, 33, 641-649.

8. J. W. Ledbetter, W. Pfleiderer and J. H. Freisheim, Photosensitized reduction of l-biopterin in the active ternary complex of dihydrofolate reductase, Photochemistry and Photobiology, 1995, 62, 71-81.

9. A. H. Thomas, C. Lorente, A. L. Capparelli, C. G. Martínez, A. M. Braun and E. Oliveros, Singlet oxygen $\left({ }^{1} \Delta_{\mathrm{g}}\right)$ production by pterin derivatives in aqueous solutions, Photochemical and Photobiological Sciences, 2003, 2, 245-250.

10. I. Fridovich, Superoxide radicals, superoxide dismutases and the aerobic lifestyle, Photochemistry and Photobiology, 1978, 28, 733-741.

11. R. Kuciel and A. Mazurkiewicz, Formation and detoxification of reactive oxygen species, Biochemistry and Molecular Biology Education, 2004, 32, 183-186.

12. J. Kagan, M. Bazin and R. Santus, Photosensitization with [alpha]-terthienyl: The formation of superoxide ion in aqueous media, Journal of Photochemistry and Photobiology B: Biology, 1989, 3, 165-174. 
13. R. W. Tuveson, G.-R. Wang, T. P. Wang and J. Kagan, Light-dependent Cytotoxic Reactions of Anthracene, Photochemistry and Photobiology, 1990, 52, 993-1002.

14. F. A. Villamena, Superoxide radical anion adduct of 5,5-dimethyl-1-pyrroline N-Oxide. 5. thermodynamics and kinetics of unimolecular decomposition, Journal of Physical Chemistry A, 2009, 113, 6398-6403.

15. M. L. Dántola, M. Vignoni, C. González, C. Lorente, P. Vicendo, E. Oliveros and A. H. Thomas, Electrontransfer processes induced by the triplet state of pterins in aqueous solutions, Free Radical Biology and Medicine, 2010, 49, 1014-1022. 
96 Capítulo 6 


\section{Capítulo 7}

\section{Caracterización del Intermediario Rojo}

Como ya se describió en el capítulo anterior, ciertos compuestos pterínicos aromáticos generan, bajo irradiación UV-A y en ausencia de $\mathrm{O}_{2}$, un compuesto color rojo y muy reactivo al cual se lo ha denominado arbitrariamente intermediario rojo (IR). En este capítulo se resumen los resultados del estudio de dicho compuesto, dirigidos a establecer su estructura química y a determinar su papel en el mecanismo de reacción de biopterina (Bip) y neopterina (Nep).

Todas las irradiaciones se realizaron en atmósfera de argón (Sección 4.2.4), condición necesaria para poder obtener el intermediario. Además de Bip y Nep, se presentan resultados de 6-hidroximetilpterina (Hmp), ya que este compuesto también genera un intermediario de características espectrales y reactividad similares a los que se generan a partir de Bip y Nep.

\subsection{Análisis de la estructura del intermediario por RMN}

Debido a que el IR es muy reactivo frente al $\mathrm{O}_{2}$, fue imposible utilizar técnicas como HPLC y espectrometría de masa para estudiarlo, ya que con estas técnicas y en las condiciones de trabajo utilizadas, es imposible evitar la oxidación de este compuesto. Por lo tanto, se recurrió a la resonancia magnética nuclear (RMN), técnica que permite el análisis de la muestra en condiciones de anaerobiosis. Para esto, se prepararon soluciones alcalinas de Bip y Nep en $\mathrm{D}_{2} \mathrm{O}$ (Sección 4.2.3) de concentración $\sim 2$ mM y se registraron los espectros de ${ }^{1} \mathrm{H}-\mathrm{RMN}$. Los estudios de $\mathrm{RMN}$ se realizaron solo en medio alcalino, debido a que en estas condiciones se logró una concentración de Bip y Nep adecuada para obtener espectros con relación señal/ruido aceptable, lo cual no fue logrado para soluciones ácidas.

Como se aprecia en la Figura 7.1, el espectro de ${ }^{1} \mathrm{H}-\mathrm{RMN}$ de una solución patrón de Bip muestra 4 señales de protones con intensidades relativas, medidas como las integrales de los picos, de 1:1:1:3. Estas señales se observan a distintos campos $(\delta, \mathrm{ppm})$ : $\delta=8,65$ (s) correspondiente al hidrógeno aromático de $\mathrm{C} 7, \mathrm{y} \delta=4,75$ (d, J $\mathrm{J}_{\alpha \beta}=5,5 \mathrm{~Hz}$ ), 4,19 (m), 1,19 (d, $\left.\mathrm{J}_{\beta \gamma}=6,5 \mathrm{~Hz}\right)$, correspondientes a los hidrógenos alifáticos $\mathrm{H} \alpha, \mathrm{H} \beta$ y $\mathrm{H} \gamma$, 
respectivamente. En el caso de Nep, también se aprecia la señal del hidrógeno aromático a $\delta=8,66$ (s) y las señales de los hidrógenos de la cadena aparecen a $\delta=4,87\left(\mathrm{~d}, \mathrm{~J}_{\alpha, \beta}=7\right.$

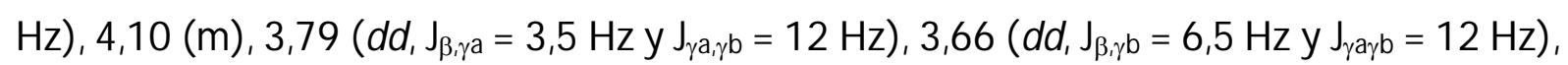
correspondientes a los hidrógenos alifáticos $\mathrm{H} \alpha, \mathrm{H} \beta, \mathrm{H} \gamma \mathrm{a}$ y $\mathrm{H} \gamma \mathrm{b}$, respectivamente. Cabe destacar que, debido a la presencia de una fuerte señal a $\delta=4,79$ correspondiente a $\mathrm{H}_{2} \mathrm{O}$, las señales a $\delta=4,75$ y $\delta=4,87$, de Bip y Nep, respectivamente, aparecen superpuestas con la señal de $\mathrm{H}_{2} \mathrm{O}$.

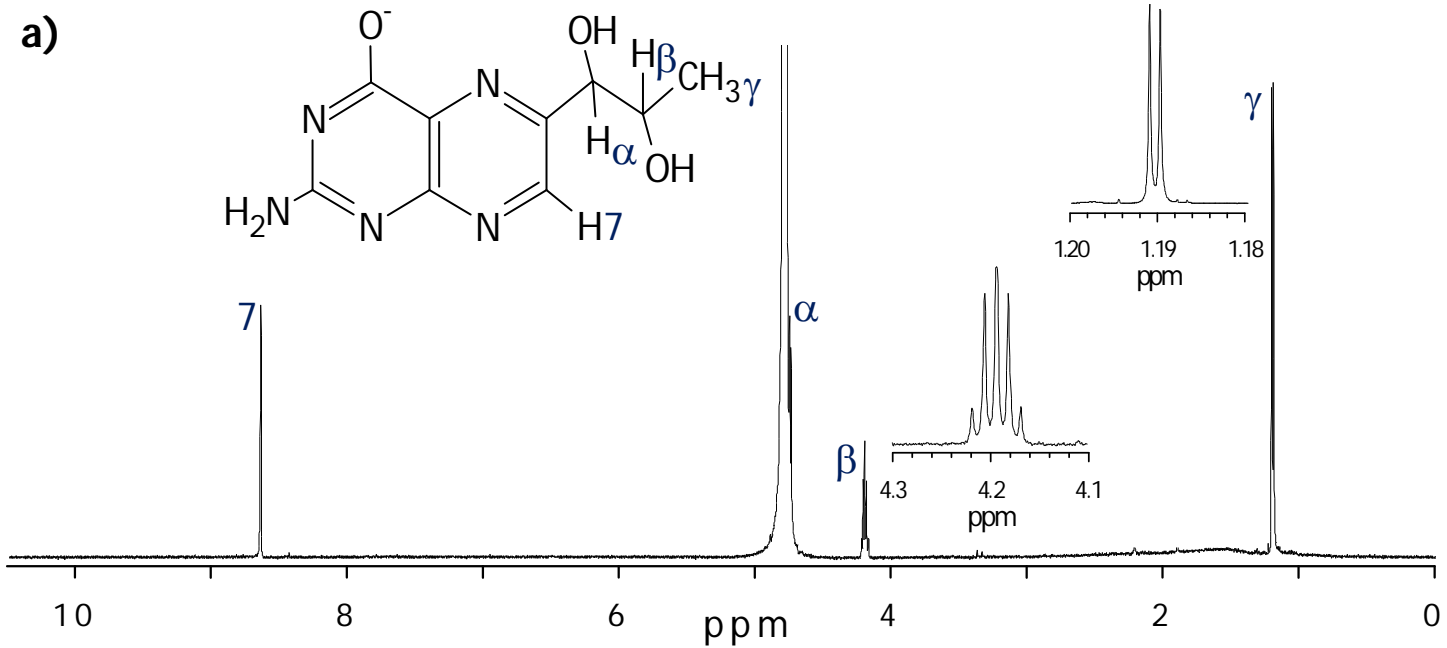

b)

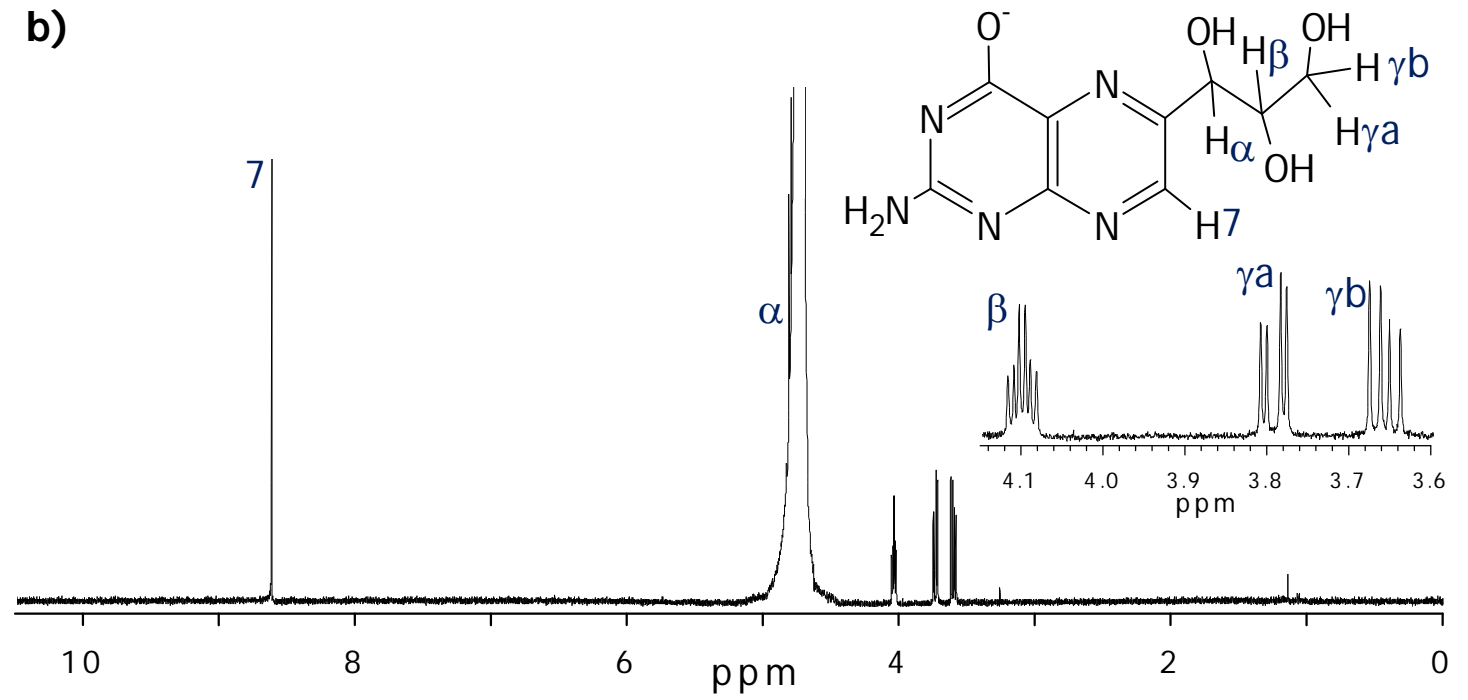

Figura 7.1 Espectros de ${ }^{1} \mathrm{H}-\mathrm{RMN}$. a) $\left[\mathrm{Bip}_{0}=2170 \mu \mathrm{M}, \mathrm{pD}=10,9 \mathrm{~b}\right)[\mathrm{Nep}]_{0}=2000 \mu \mathrm{M}, \mathrm{pD}=$ 10,2 .

Para analizar el IR, las soluciones de Bip y Nep fueron saturadas en Ar y luego irradiadas por 20 min (Sistema de irradiación I, $350 \mathrm{~nm}$ ). Al interrumpir la irradiación se observó un color rojo intenso en las soluciones, indicando la formación del compuesto buscado. Rápidamente, se registraron los espectros ${ }^{1} \mathrm{H}-\mathrm{RMN}$ donde se observó la aparición de nuevas señales, además de las residuales correspondientes al remanente de 
reactivo, las cuales se aprecian en la Figura 7.2. Es importante aclarar que de las nuevas señales, dos son exactamente las mismas para Bip y Nep, mientras que las restantes varían levemente.

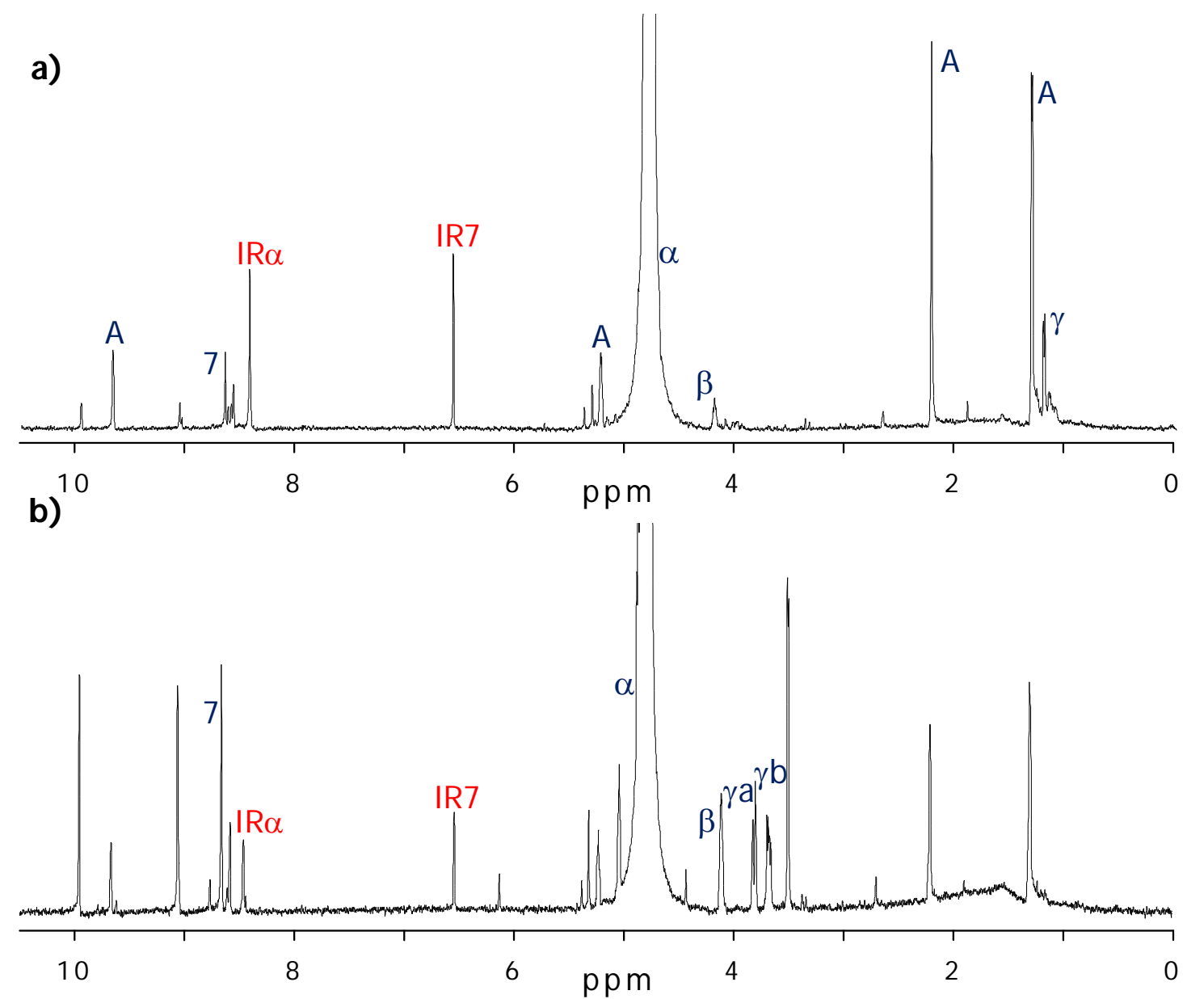

Figura 7.2 Espectros de 1H-RMN de las soluciones expuestas en la Figura 7.1, irradiadas por 20 min en atmósfera de Ar. a) Bip, b) Nep

A continuación, estas mismas soluciones se pusieron en contacto con el aire. Esto condujo a la desaparición del color rojo, debido al consumo del IR (ver reacción térmica descripta en la Sección 6.2), tornándose incoloras nuevamente. Los espectros ${ }^{1} \mathrm{H}-\mathrm{RMN}$ registrados después de airear las soluciones irradiadas, muestran claramente la desaparición de las dos señales intensas equivalentes para Bip y $\operatorname{Nep}(\delta=8,42$ (s) y 6,58 (s)). Asimismo, se observa que no varían las otras señales que aparecen con la irradiación y que, además, aumenta la intensidad de tres señales débiles en los espectros tomados en ausencia de $\mathrm{O}_{2}$. Estas tres señales son las mismas para Bip y Nep, y aparecen a $\delta=9,95$ (s) y 9,05 (s), las dos de mayor intensidad, mientras que a $\delta=9,03$ (s) se detecta la tercera que es prácticamente despreciable (Figura 7.3). 


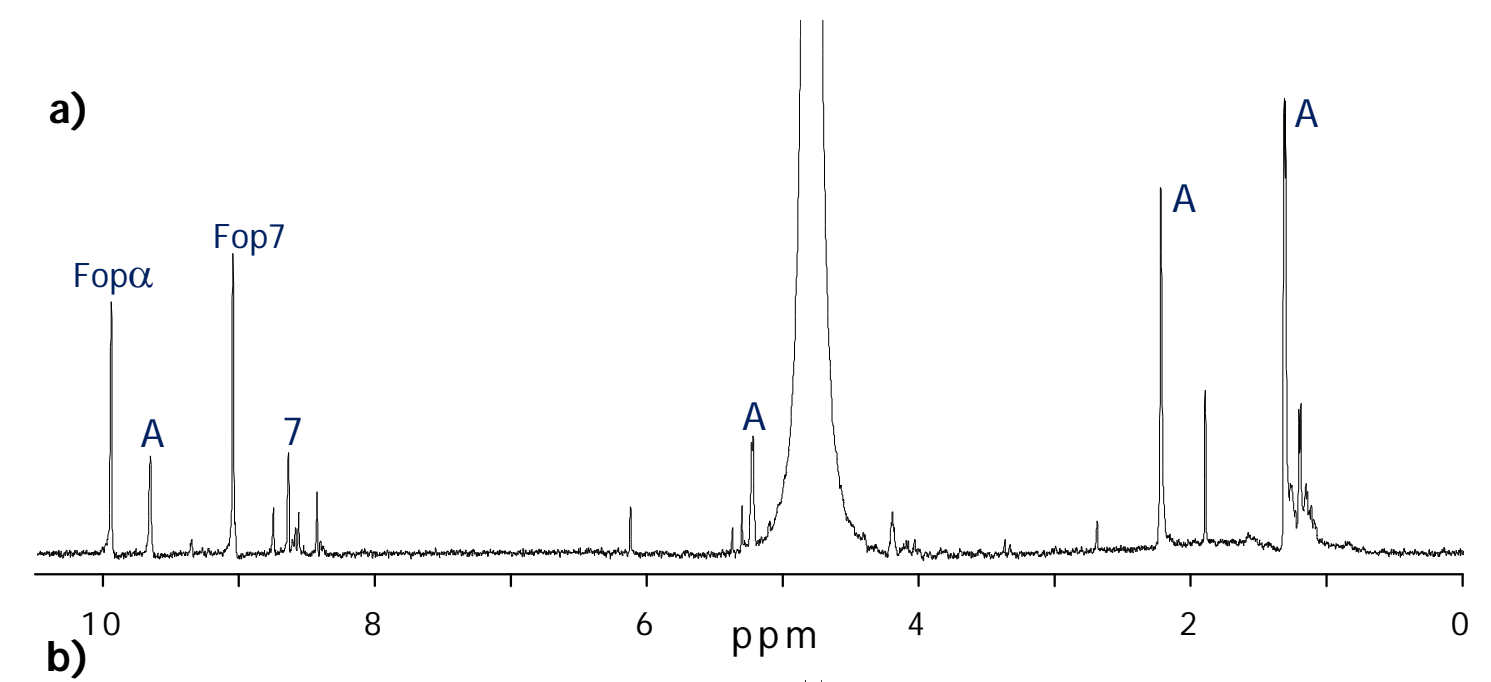

b)

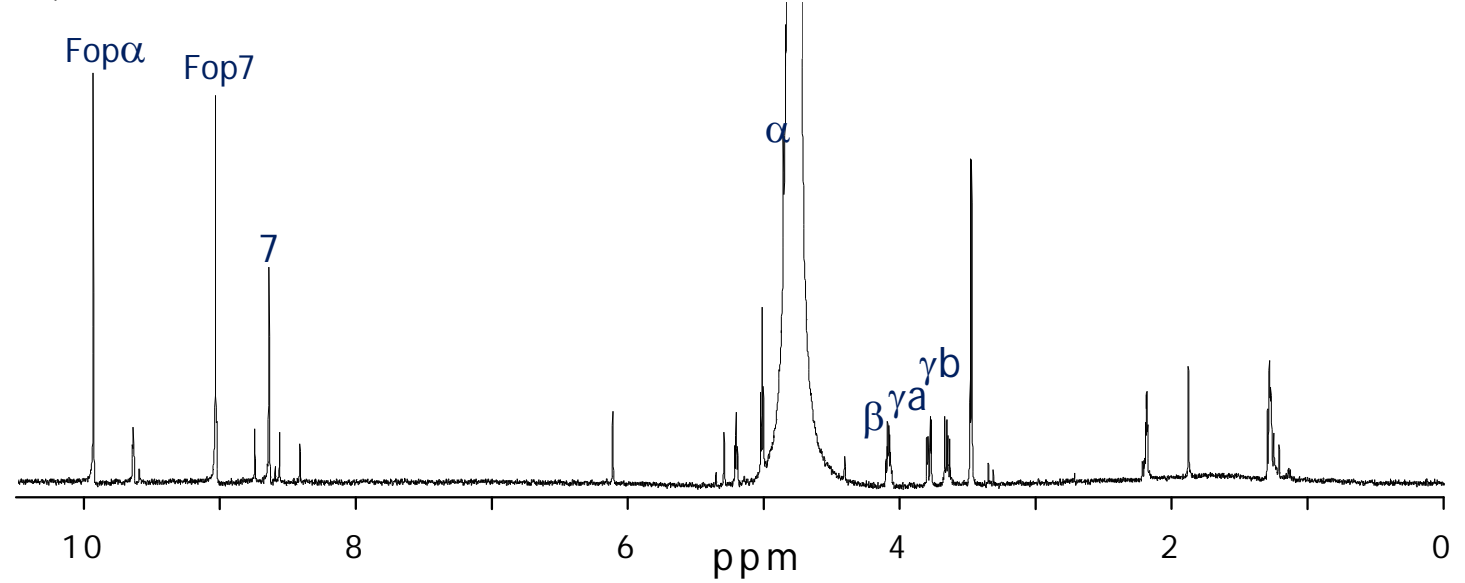

Figura 7.3 Espectros de 1H-RMN de las soluciones expuestas en la Figura 7.1, irradiadas por 20 min en atmósfera de Ar y luego aireadas. a) Bip, b) Nep

Teniendo en cuenta todo lo expuesto en el capítulo anterior, las dos señales que desaparecen deben corresponder al intermediario que fue oxidado durante la reacción en oscuridad por el $\mathrm{O}_{2}$. Por otro lado, las señales que aparecen durante la fotólisis y no desaparecen con la admisión de aire, ya sea para el caso de Bip como para Nep, deben corresponder a fotoproductos estables en presencia de $\mathrm{O}_{2}$. Finalmente, los picos que aparecen o se intensifican al airear las soluciones irradiadas en anaerobiosis deben corresponder a 6-formilpterina (Fop) y 6-carboxipterina (Cap), que son los productos de oxidación del intermediario, como se explicó en el capítulo anterior.

Esto fue confirmado realizando los espectros ${ }^{1} \mathrm{H}-\mathrm{RMN}$ de soluciones en $\mathrm{D}_{2} \mathrm{O}$ de estas dos pterinas aromáticas, donde a Fop le corresponden 2 señales a $\delta=9,95$ (s) y 9,05 (s) y a Cap una señal a $\delta=9,03$ (s) (Figura 7.4.a y b). Por lo tanto, y en concordancia con el esquema de reacción propuesto por Pfleiderer et al., estos resultados demuestran que Fop es el producto de la reacción del IR con $\mathrm{O}_{2} \mathrm{y}$, por ende, proveen evidencia adicional que sostiene el mecanismo propuesto en el Capítulo 6 (Esquema 6.4). La señal 
observada para Cap fue muy pequeña, incluso, hasta en algunos casos, no fue detectada. La formación de Cap corresponde a la presencia de trazas de $\mathrm{O}_{2}$ en el tubo de reacción durante la irradiación, y se genera en una muy baja concentración, como ya se observó por HPLC en la Sección 6.2.

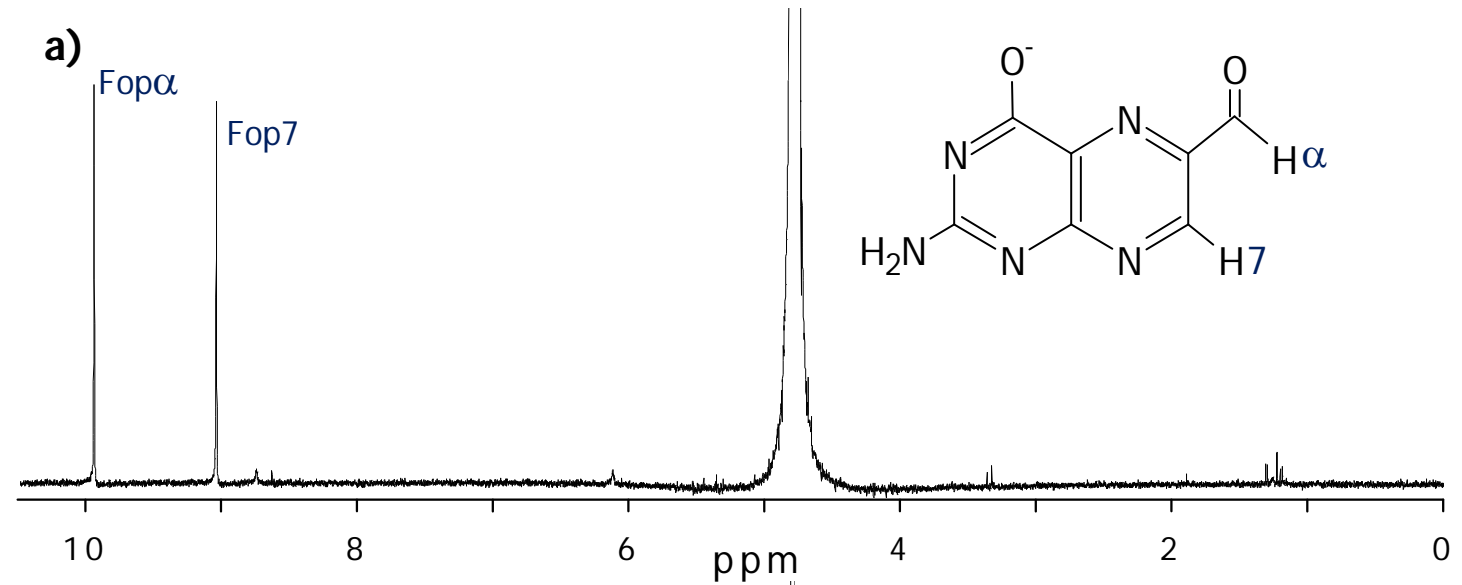

b)

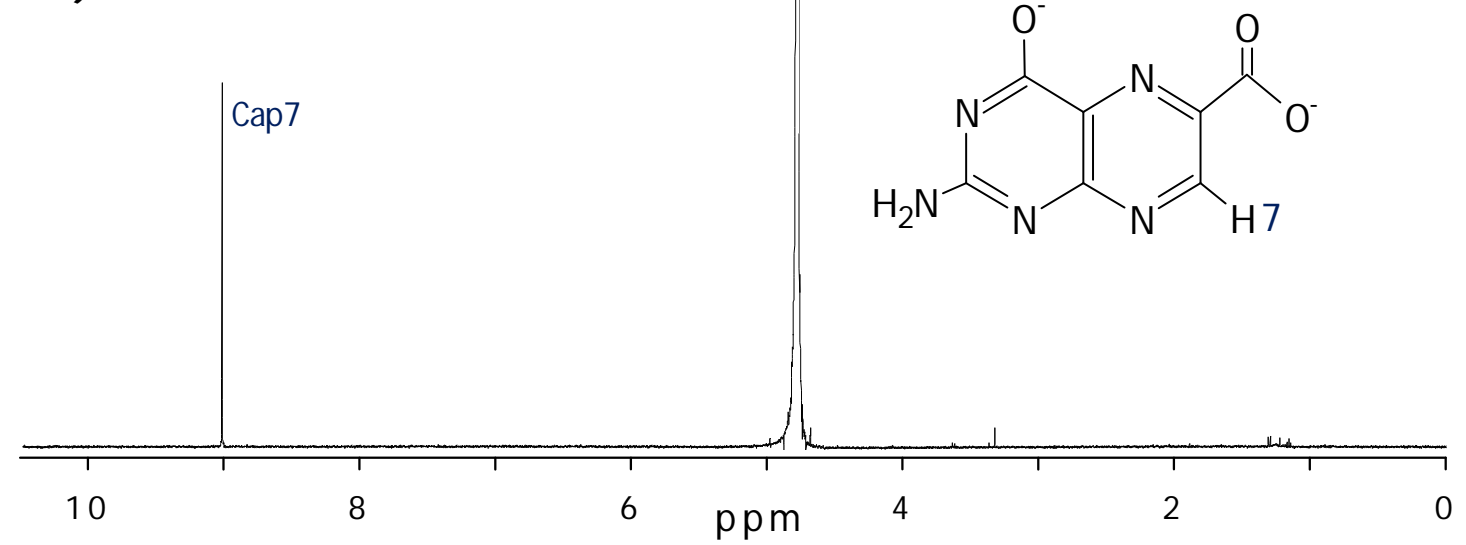

c)<smiles>CC=CCC(C)O</smiles>

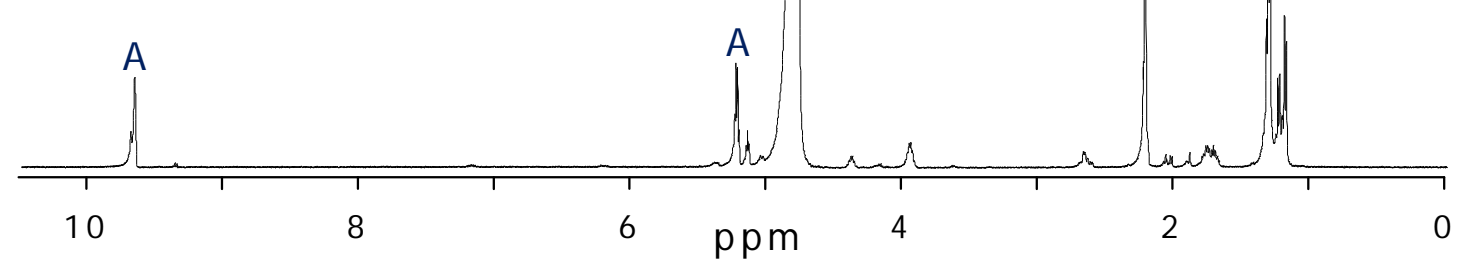

Figura 7.4 Espectros de ${ }^{1} \mathrm{H}-\mathrm{RMN}$ de las soluciones patrones de a) Fop, b) Cap, c) acetaldehído.

Se realizaron estudios adicionales para investigar cuáles son los productos generados en la irradiación y estables en presencia de $\mathrm{O}_{2}$. Suponiendo que la molécula de Bip se fragmenta en el enlace entre $\mathrm{C} \alpha$ y $\mathrm{C} \beta$ como consecuencia de la reacción 
fotoquímica que produce el IR, el otro producto formado debe ser un compuesto con dos átomos de carbono. El compuesto más probable sería el acetaldehído, del cual se registró el espectro ${ }^{1} \mathrm{H}-\mathrm{RMN}$ en $\mathrm{D}_{2} \mathrm{O}$. Como se muestra en la Figura 7.4.C, las señales principales que corresponden a este compuestos se observan a $\delta=9,66$ y 2,22 mientras que también se pueden apreciar los picos correspondientes al producto originado de la adición aldólica, el 3-hidroxibutanal $(\delta=5,23$ y 1,32$) .{ }^{1}$ Estas señales están presentes en el espectro de la solución irradiada de Bip, tanto antes (Figura 7.2.a) como después de la ingreso de aire en el tubo de reacción (Figura 7.3.a). De esta manera, se confirma la rotura y separación de la cadena unida al C6 de Bip, con la consecuente formación de acetaldehído. Vale la pena insistir que la escisión de la cadena sucede durante la irradiación y previamente a la incorporación de $\mathrm{O}_{2}$, lo cual explica por qué la intensidad de dichas señales no se modifica al airear la solución.

En el caso de Nep, también se observaron señales en el espectro de ${ }^{1} \mathrm{H}-\mathrm{RMN}$ que se forman durante la irradiación anaeróbica y luego su intensidad no se modifica con la entrada de $\mathrm{O}_{2}$. Más aún, estas señales son compatibles con compuestos de dos átomos de carbono pero los valores de $\delta$ en este espectro, difieren de los de valores obtenidos para acetaldehído y 3-hidroxibutanal. Esto es esperable considerando que las estructuras químicas de los sustituyentes de Bip y Nep son diferentes. La identificación del/ de los compuesto/s responsable/s de estas señales no se pudo llevar a cabo porque los patrones correspondientes no estuvieron disponibles.

Por lo tanto, sabiendo entonces que la reacción fotoquímica de Bip y Nep en ausencia de $\mathrm{O}_{2}$ genera un producto de dos átomos de carbono, el otro producto formado, es decir el IR, debe poseer la estructura base de las pterinas (doble anillo heterocíclico) con un sustituyente de un solo átomo de carbono. Los valores de las señales correspondientes al intermediario, muestran que este compuesto ya no posee hidrógenos aromáticos, debido a que estos aparecen a bajos campos ( $\delta \sim 8)$, con lo cual la pérdida de aromaticidad sucede en el anillo pirazínico. Por lo tanto, la señal detectada a $\delta=6,58$ puede atribuirse a la presencia de un hidrógeno olefínico en el C7. Si el anillo se reduce entonces, considerando la falta de $\mathrm{O}_{2}$, el sustituyente con un solo átomo de $\mathrm{C}$ debería estar oxidado. Esto concuerda con la señal observada a $\delta=8,42$, que puede asignarse al hidrógeno del carbono aldehído que extiende la conjugación con el sistema olefínico. Por lo tanto, estos resultados se correlacionan correctamente con la estructura 
química del 6-formil-5,8-dihidropterina previamente propuesta por Pfleiderer (Figura $7.5)^{2}$

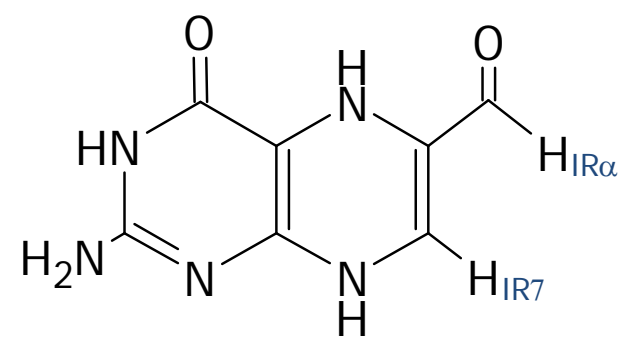

Figura 7.5 Estructura del intermediario: 6-formil-5,8-dihidropterina.

\subsection{Análisis de los fotoproductos de 6-hidroximetilpterina por RMN}

Como bien se mencionó en el Capítulo 3, el comportamiento fotoquímico en solución acuosa de Hmp es similar al observado para Bip y Nep. Es decir, cuando se irradia una solución de $\mathrm{Hmp}$ en ausencia de $\mathrm{O}_{2}$, se genera un intermediario que es oxidado rápidamente al ponerse en contacto con el $\mathrm{O}_{2}$, obteniéndo Fop como producto final. 3 Dicho intermediario tiene las mismas características espectrales que el IR descripto para Bip y Nep. Sabiendo que Bip y Nep también generan Fop como producto final y que, por ende, el mecanismo de reacción podría ser el mismo para las tres pterinas, el intermediario formado a partir de Hmp debería ser el IR. Consecuentemente, se decidió estudiar soluciones de Hmp por ${ }^{1} \mathrm{H}-\mathrm{RMN}$ haciendo un análisis similar a lo descripto en la sección anterior. Es importante destacar que como Hmp posee como sustituyente un solo átomo de C, las señales correspondientes al fragmento de la cadena escindida no deberían observarse, simplificando así el análisis.

Primero se obtuvo el espectro de una solución de Hmp en medio alcalino sin irradiar (Figura 7.6.a). Este espectro posee una sola señal a $\delta=8,64$ que corresponde al H7 del anillo. La señal correspondiente a los Ha del sustituyente no se observa debido a que aparece superpuesta con la señal del $\mathrm{H}_{2} \mathrm{O}$. Luego, la solución se irradió por 60 min con el Sistema de irradiación I y se registró el espectro ${ }^{1} \mathrm{H}-\mathrm{RMN}$, donde se observa la aparición de las dos señales correspondientes al intermediario con valores de $\delta=8,42$ y $\delta=6,58$ (Figura 7.6.b). Posteriormente, se aireó la solución y se registró nuevamente el espectro. Se pudo apreciar entonces la desaparición de estas dos señales con la consecuente aparición de las señales correspondientes a Fop y Cap, siendo esta última casi despreciable (Figura 7.6.c). Dos puntos importantes se desprenden de este análisis: por un lado, los valores de $\delta$ de las señales del intermediario de Hmp son exactamente los mismos que para Bip y Nep. Por otro lado, no se observan señales adicionales al 
irradiar la solución, indicando que, como era de esperar, no se forma ningún otro producto proveniente de la ruptura del sustituyente.

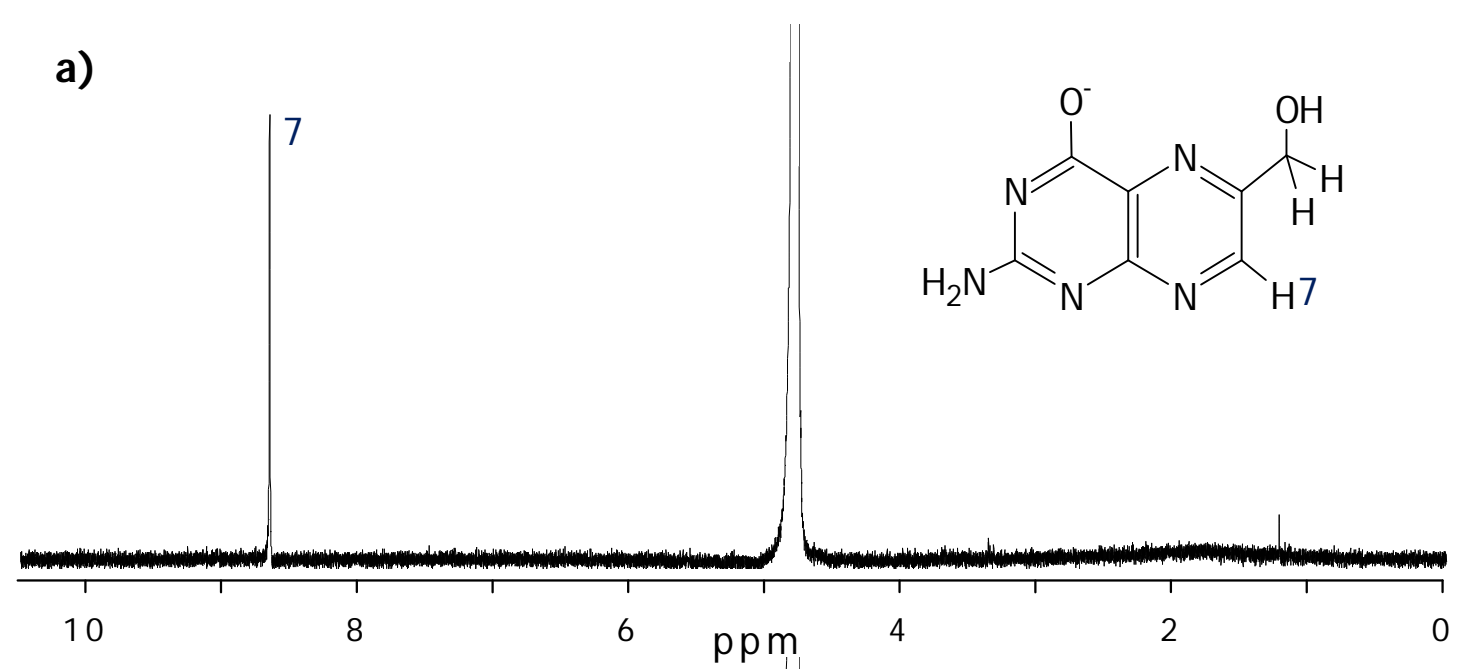

b)

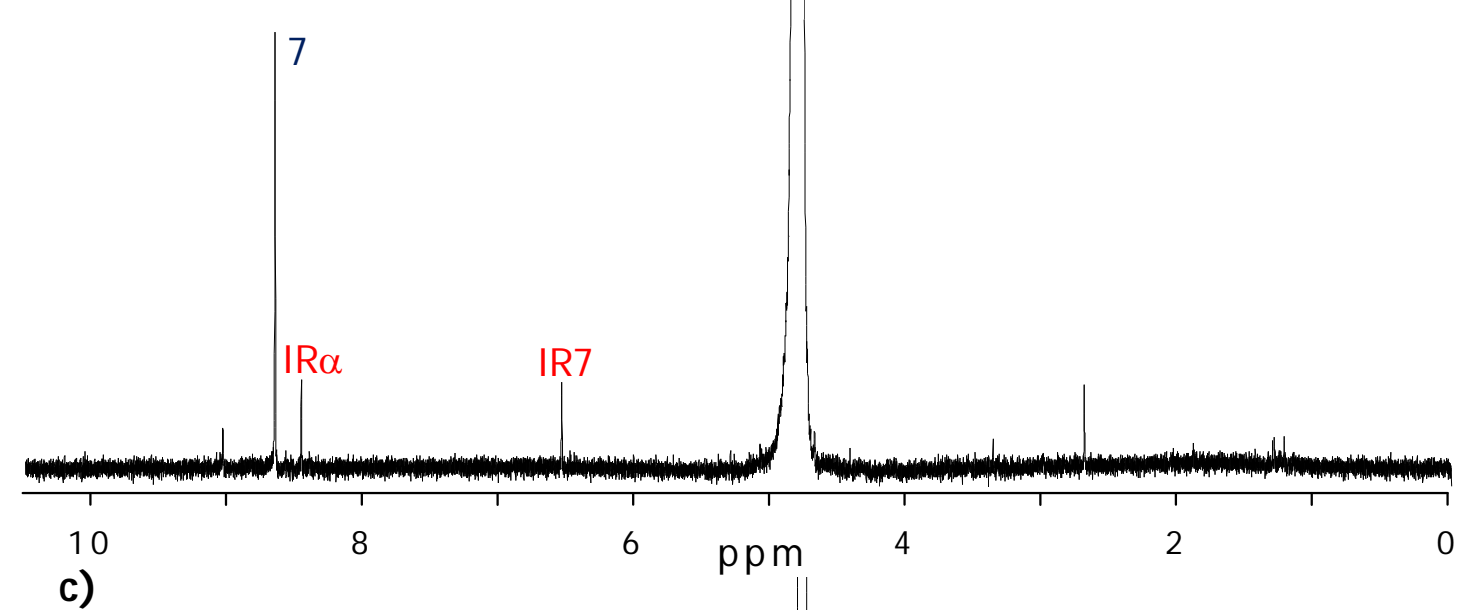

c)

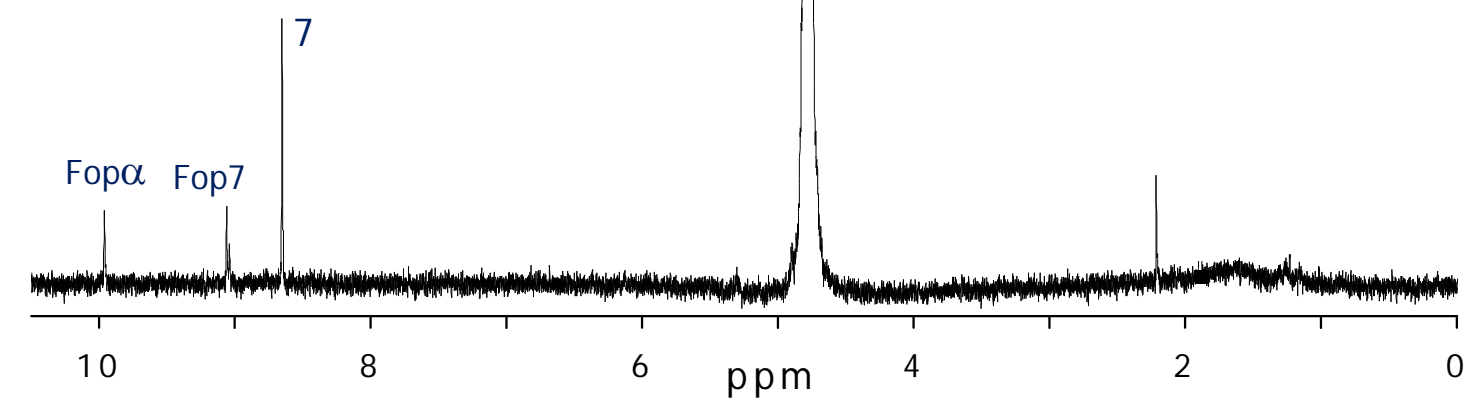

Figura 7.6 Espectros de 1H-RMN de una solución de Hmp. a) antes de irradiar, b) luego de la irradiación en atmósfera de $\mathrm{Ar}(\mathrm{ti}=60 \mathrm{~min})$, c) luego de airear la solución b).

De esta manera, se comprueba que, al irradiar una solución de Bip, Nep o Hmp en ausencia de $\mathrm{O}_{2}$, se forma el mismo compuesto IR. Asimismo, se ratifica que este IR tiene la estructura propuesta en la Figura 7.5. Por otro lado, estos resultados sugieren que la 
asignación de las señales correspondientes a los fragmentos provenientes de la escisión del sustituyente de Bip y Nep es correcta.

\subsection{Estudio de la estabilidad del intermediario}

Se sabe que el intermediario es inestable en presencia de $\mathrm{O}_{2} \mathrm{y}$, además, se ha propuesto que las 5,8-dihidropterinas son inestables en condiciones anaeróbicas y tautomerizan a 7,8-dihidropterinas. ${ }^{4}$ Por lo tanto, se decidió estudiar su estabilidad en ausencia de $\mathrm{O}_{2}$ por espectrofotometría UV-vis y HPLC. Para esto, se generó el intermediario irradiando soluciones de Bip o Nep burbujeadas previamente con Ar. Una vez finalizada la irradiación, se conservaron esas soluciones en oscuridad y en condiciones anaeróbicas por un tiempo determinado (llamado arbitrariamente "tiempo de oscuridad", to) y se registraron los espectros de absorción. Como se mencionó en el capítulo anterior, este intermediario posee una banda de absorción característica centrada en $480 \mathrm{~nm}$. En la Figura 7.7 se observa para Bip y Nep en medio ácido y alcalino, como disminuye la intensidad de esta banda hasta desaparecer por completo, indicando que el IR es inestable incluso en ausencia de $\mathrm{O}_{2}$. Por lo tanto, el intermediario no sólo reacciona con $\mathrm{O}_{2}$ para dar Fop y $\mathrm{H}_{2} \mathrm{O}_{2}$, sino que también sufre una reacción térmica independiente del $\mathrm{O}_{2}$ generando productos.

El consumo del IR en función del tiempo de oscuridad (to) tuvo, en todos los casos, un comportamiento de primer orden. A modo de ejemplo, en la Figura 7.8 se muestra la evolución temporal de la absorbancia a $480 \mathrm{~nm}$ para una solución de Bip en medio alcalino. A partir del ajuste de la ecuación no lineal $A_{t}=A_{\text {inf }}+\left(A_{0}-A_{i n f}\right) e^{-k t}$ a los datos experimentales, es posible calcular la constante de velocidad de desaparición del IR. Para esto, se realizaron experimentos a temperatura controlada y se obtuvieron los valores expuestos en la Tabla7.1. 


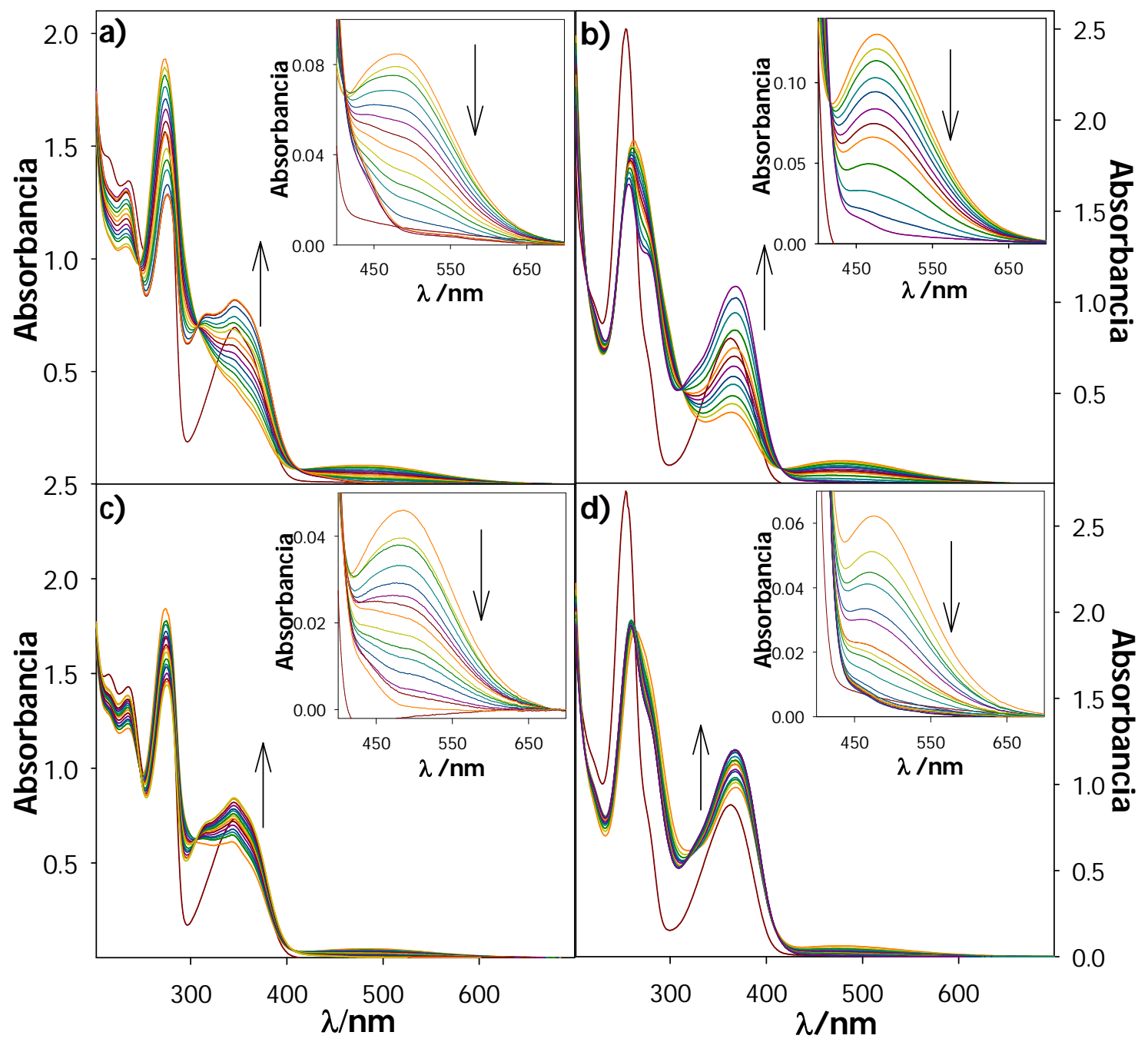

Figura 7.7 Evolución del espectro de absorción de soluciones de Bip y Nep, luego de ser irradiadas en ausencia de $\mathrm{O}_{2}$. Las soluciones se mantuvieron en condiciones anaeróbicas y en oscuridad, registrándose los espectros cada $\sim 5 \mathrm{~min}$. a) $[\mathrm{Bip}]_{0}=109 \mu \mathrm{M}, \mathrm{pH}=5,1, \mathrm{ti}=$ $4 \mathrm{~min}$, to $=120 \mathrm{~min}, \mathrm{~b})[\mathrm{Bip}]_{0}=109 \mu \mathrm{M}, \mathrm{pH}=10,5, \mathrm{ti}=2 \mathrm{~min}$, to $\left.=140 \mathrm{~min}, \mathrm{c}\right)[\mathrm{Nep}]_{0}=150$ $\mu \mathrm{M}, \mathrm{pH}=4,9, \mathrm{ti}=2 \mathrm{~min}$, to $=105 \mathrm{~min}, \mathrm{~d})[\mathrm{Nep}]_{0}=120 \mu \mathrm{M}, \mathrm{pH}=10,3, \mathrm{ti}=6 \mathrm{~min}$, to $=60 \mathrm{~min}$. Camino óptico $=1 \mathrm{~cm}$. Sistema de irradiación $\mathrm{I}$.

\begin{tabular}{ccc}
\hline & \multicolumn{2}{c}{$\mathbf{k} / \mathbf{m i n}^{-1}$} \\
medio ácido & medio alcalino \\
\hline Nep & $0,028 \pm 0,006$ & $0,027 \pm 0,006$ \\
\hline
\end{tabular}

Tabla 7.1 Constantes de velocidad de desaparición del IR a $28^{\circ} \mathrm{C}$. 


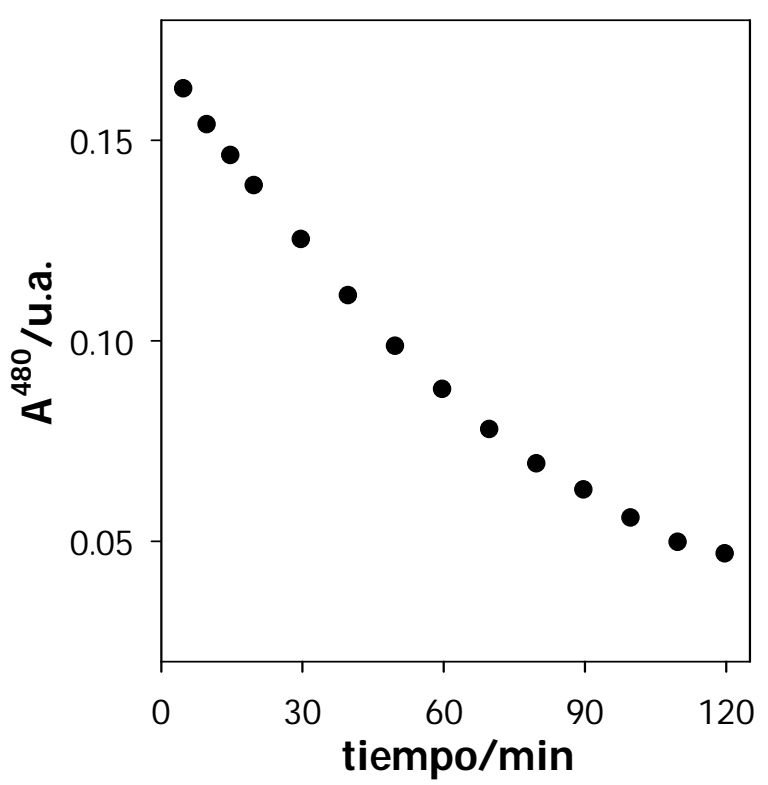

Figura 7.8 Evolución de la absorbancia a $480 \mathrm{~nm}$ en función del tiempo de oscuridad, para una solución de Bip $\left([\mathrm{Bip})_{0}=150 \mu \mathrm{M}, \mathrm{pH}=10,4, \mathrm{ti}=6 \mathrm{~min}\right.$, to $\left.=120 \mathrm{~min}\right)$. Sistema de irradiación I.

Claramente se observa que para Bip en ambos medios y Nep en medio ácido, el valor de la constante de desaparición es muy similar. Sin embargo, para Nep en medio alcalino el valor obtenido es entre 3 y 4 veces mayor. Los mismos estudios se realizaron para Hmp y se encontró que el IR también es inestable y sufre el mismo comportamiento que para Bip y Nep, con un valor de constante muy parecido a las constantes ya mencionadas $\left(\mathrm{k}=0,042 \pm 0,008 \mathrm{~min}^{-1}\right.$, en medio alcalino a $\left.25^{\circ} \mathrm{C}\right)$.

Teniendo en cuenta que la reacción térmica tiene lugar en ausencia de $\mathrm{O}_{2}$, es de esperar que no se genere $\mathrm{H}_{2} \mathrm{O}_{2}$ como producto. Para comprobar esto, se realizó otra serie de experimentos en los cuales soluciones de Bip y Nep de concentración entre 140$200 \mu \mathrm{M}$, fueron irradiadas previo burbujeo con $\mathrm{Ar}$ ( $\mathrm{pH}=5,5$ y 10,5). Una vez finalizada la irradiación, las soluciones se conservaron en oscuridad por distintos períodos de tiempo, burbujeadas con Ar periódicamente para garantizar la ausencia de $\mathrm{O}_{2}$. Finalmente, se burbujearon con aire y se determinó la concentración de $\mathrm{H}_{2} \mathrm{O}_{2}$. Como se puede apreciar en la Figura 7.9, para ambos compuestos y en las dos condiciones de pH, la cantidad de $\mathrm{H}_{2} \mathrm{O}_{2}$ formado disminuye en función del tiempo transcurrido desde la interrupción de la irradiación y el ingreso de $\mathrm{O}_{2}$ en la celda. Estos resultados confirman que la reacción térmica independiente de $\mathrm{O}_{2}$ no genera $\mathrm{H}_{2} \mathrm{O}_{2}$ como producto. 

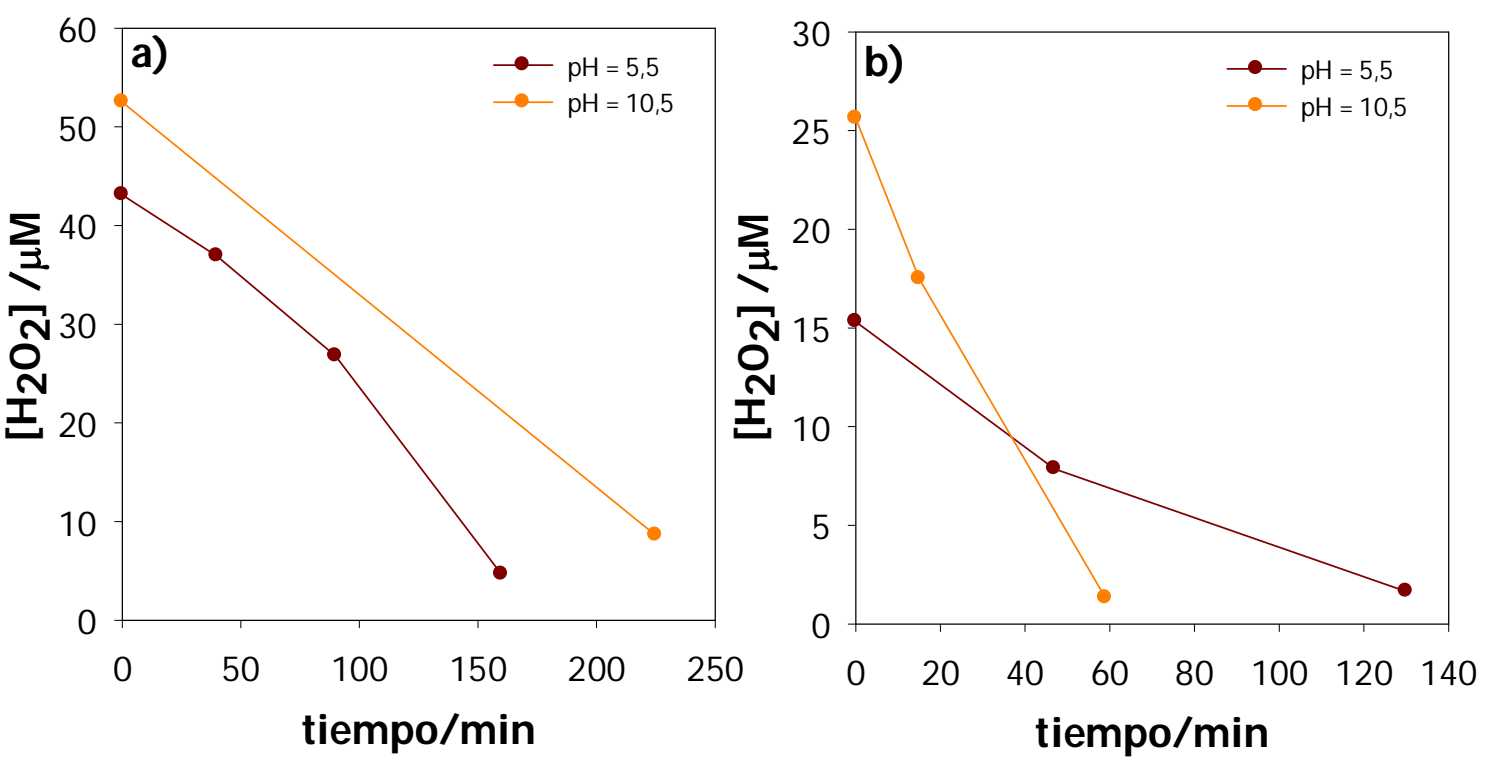

Figura 7.9 Concentración de $\mathrm{H}_{2} \mathrm{O}_{2}$ en soluciones de Bip y Nep irradiadas en ausencia de $\mathrm{O}_{2}$, y conservadas en oscuridad en ausencia de $\mathrm{O}_{2}$. A distintos tiempos se burbujeó aire por 2 min y se determinó la concentración de $\mathrm{H}_{2} \mathrm{O}_{2}$. El tiempo del eje $\mathrm{X}$ corresponde al período transcurrido desde la interrupción de la irradiación hasta el ingreso de aire en la celda. a) $\left.[\text { Bip }]_{0}=150 \mu \mathrm{M}, \mathrm{b}\right)[\mathrm{Nep}]_{0}=150 \mu \mathrm{M}$. Sistema de irradiación I.

Para determinar los productos generados durante la reacción en oscuridad independiente de $\mathrm{O}_{2}$ descripta anteriormente, las muestras se analizaron por HPLC. En la Figura 7.10, se muestran los cromatogramas de soluciones de Bip en ambos medios, irradiadas en ausencia de $\mathrm{O}_{2} \mathrm{y}$, luego conservadas en oscuridad hasta finalizar la reacción térmica. Se aprecia claramente que el producto mayoritario es Fop, al igual que en las irradiaciones en aire o en ausencia de $\mathrm{O}_{2}$ pero abiertas al aire inmediatamente después de finalizada la irradiación. Esto no concuerda con la hipótesis postulada previamente por Pfleiderer que dice que las 5,8-dihidropterinas tautomerizan a las correspondientes 7,8-dihidropterinas. ${ }^{4}$ En este caso se encontró como principal producto el derivado oxidado, y sólo una pequeña proporción de 6-formil-7,8dihidropterina $\left(\mathrm{H}_{2} \mathrm{Fop}\right)(<10 \%)$. Asimismo, aparecen otros dos productos a mayor tiempo de retención ( $\operatorname{tr}=18,5$ y 21,0 $\mathrm{min}$ ). Ambos productos poseen espectros de absorción muy similares entre sí y también muy parecidos a los espectros de Bip, Nep y Hmp en medio ácido (Figura 2.5, Capítulo 2). Esto hace suponer que la estructura pterínica de estos compuestos desconocidos, puede ser aromática y que quizás poseen un $\mathrm{OH}$ unido al $\mathrm{C} \alpha$ como sustituyente en el $\mathrm{C} 6$, como es el caso de los reactivos mencionados. Para Nep se obtuvieron cromatogramas similares. 


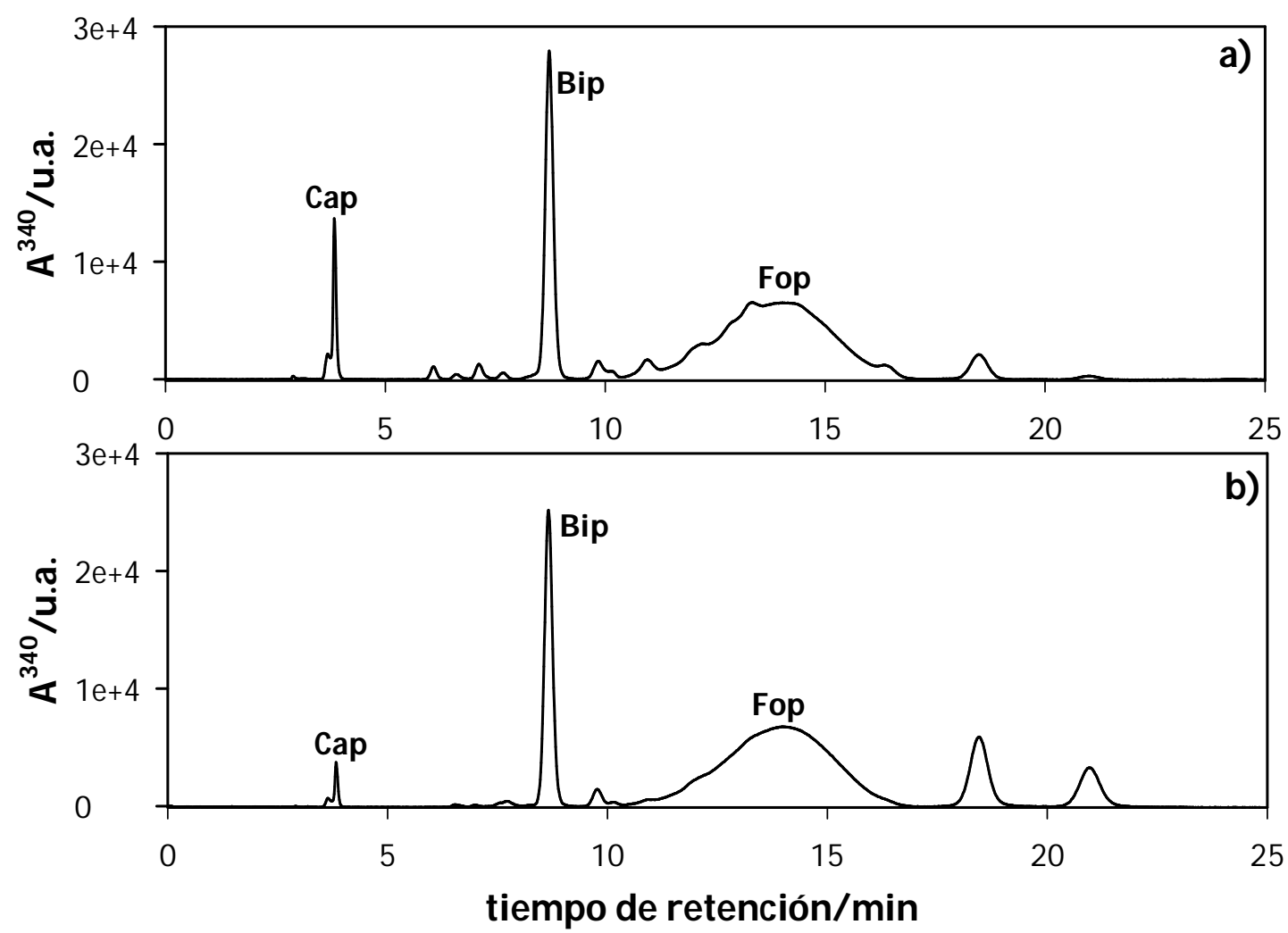

Figura 7.10 Cromatogramas $\left(\lambda_{\mathrm{an}}=340 \mathrm{~nm}\right)$ de soluciones irradiadas en ausencia de $\mathrm{O}_{2} \mathrm{y}$ conservadas en anaerobiosis y en la oscuridad hasta consumirse el IR. a) [Bip] $]_{0}=109 \mu \mathrm{M}$, $\mathrm{pH}=5,1, \mathrm{ti}=4 \mathrm{~min}$, to $=120 \mathrm{~min}, \mathrm{~b})[\mathrm{Bip}]_{0}=109 \mu \mathrm{M}, \mathrm{pH}=10,5, \mathrm{ti}=2 \mathrm{~min}$, to $=140 \mathrm{~min}$. Sistema de irradiación I y Equipo HPLC I.

Sabiendo que el producto mayoritario de la reacción térmica es Fop, para ambos reactivos y en las dos condiciones de pH estudiadas, se realizó un balance de masa para cuantificar la cantidad de reactivo que se transforma en este producto $(\Delta \mathrm{Fop} / \Delta \mathrm{R})$, encontrándose un valor entre 60 - 70\%. Por otro lado, se podría suponer que la oxidación del IR a Fop se produce por un lento ingreso de $\mathrm{O}_{2}$ a la celda. Sin embargo, esto se puede descartar ya que no se detecta $\mathrm{H}_{2} \mathrm{O}_{2}$ cuando se completa la descomposición térmica del IR. Si bien falta información para proponer una hipótesis consistente, se podría especular con procesos de dismutación en los cuales el IR se oxide a Fop y se reduzca a otros productos.

Para avanzar con el análisis de los productos desconocidos, se determinaron los pesos moleculares por espectrometría de masa (Equipo MS I, Sección 5.4). Para ambos productos se encontró el mismo ión molecular con $\mathrm{m} / \mathrm{z}=385$, correspondiente a $[\mathrm{M}+\mathrm{H}]^{+}$. Además, se observan dos picos en $\mathrm{m} / \mathrm{z}=407$ y 367, correspondiente a [M+Na]+ y $\left[\mathrm{M}-\mathrm{H}_{2} \mathrm{O}+\mathrm{H}\right]^{+}$, respectivamente. Este ión molecular podría corresponder a dos estructuras pterínicas unidas entre sí. Por otra parte, estos dos productos desconocidos 
poseen igual espectro de absorción y mismo peso molecular, de manera que podrían ser dos isómeros.
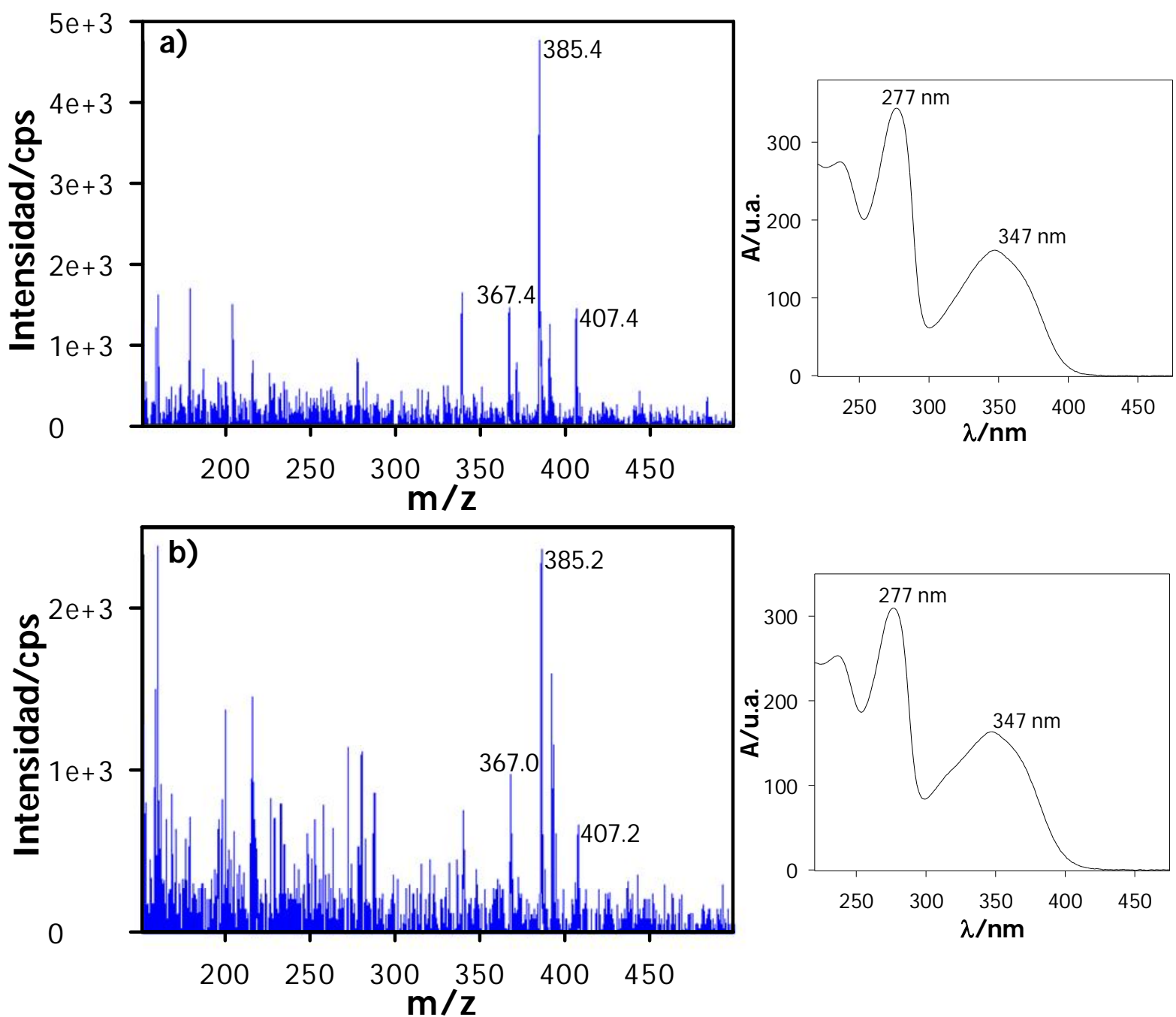

Figura 7.11 Espectros de masa y de absorción de los productos de la reacción térmica. a) producto 1, b) producto 2 .

\subsection{Conclusiones}

A partir del análisis por ${ }^{1} \mathrm{H}-\mathrm{RMN}$ se pudo establecer que en la fotólisis de Bip y Nep en ausencia de $\mathrm{O}_{2}$, ya sea en medio ácido o alcalino, se forma el mismo intermediario. Este compuesto es 6-formil-5,8-dihidropterina, estructura que había sido sugerida previamente por Pfleiderer et al.. ${ }^{2}$ Además, se confirmó que al irradiar Hmp también se forma este mismo intermediario. Por otro lado, se estudió la estabilidad de este compuesto, los productos generados y se determinaron las correspondientes constantes de velocidad de desaparición del IR. Se concluyó entonces que el IR no es estable, aún en ausencia de $\mathrm{O}_{2}$, y que genera Fop y otros productos que no pudieron ser 
caracterizados correctamente. El Esquema 7.1 resume las reacciones estudiadas hasta el momento.<smiles>CC(O)C(O)c1cnc2nc(N)[nH]c(=O)c2n1</smiles>

Bip<smiles>Nc1nc2ncc(CO)nc2c(=O)[nH]1</smiles>

Hmp<smiles></smiles><smiles></smiles><smiles>CC(C)C([18OH])[13CH2][13CH3]</smiles><smiles>CCCCCCCCC</smiles><smiles>CC1CCCCC1C(=O)OC=N</smiles>
Nep

Fop

\section{Esquema 7.1 Estructura del IR y reacciones en las que participa.}

\subsection{Referencias}

1. B. Reinhard, 10 - Chemistry of the Alkaline Earth Metal Enolates, in Advanced Organic Chemistry, Academic Press, San Diego, 2002, pp. 373-434.

2. R. Mengel, W. Pfleiderer and W. R. Knappe, Norrish type II analogous photodegradation of neopterin and biopterin to 5,8 -dihydropterin-6-aldehyde, Tetrahedron Lett., 1977, 18, 2817-2820.

3. F. M. Cabrerizo, A. H. Thomas, C. Lorente, M. L. Dántola, G. Petroselli, R. Erra-Balsells and A. L. Capparelli, Generation of Reactive Oxygen Species during the Photolysis of 6-(Hydroxymethyl)pterin in Alkaline Aqueous Solutions, Helvetica Chimica Acta, 2004, 87, 349-365.

4. W. Pfleiderer, Chemistry and properties of dihydropterins, in Biochemical and Clinical Aspects of Pteridines, ed. W. Pfleiderer, H. Wachter and A. J. Blair, Walter de Gruyter \& Co., Berlin, New York, 1987, pp. 3-21. 
112 Capítulo 7 


\section{Capítulo 8}

\section{Fotoquímica de 7,8-dihidroneopterina}

En este capítulo se presenta un estudio de la fotoquímica de 7,8dihidroneopterina $\left(\mathrm{H}_{2} \mathrm{Nep}\right)$ en solución acuosa bajo irradiación UV-A. No existen antecedentes en literatura sobre este tema, a pesar de la gran relevancia biológica de este compuesto. A diferencia de lo que ocurre con los derivados oxidados, el comportamiento fotoquímico de $\mathrm{H}_{2} \mathrm{Nep}$ es sustancialmente distinto al de 7,8dihidrobiopterina ( $\left.\mathrm{H}_{2} \mathrm{Bip}\right)$. Debido a este hecho, los resultados obtenidos para ambos compuestos se presentan en dos capítulos diferentes. Como se entenderá más adelante, la fotoquímica de $\mathrm{H}_{2}$ Bip es más compleja, motivo por el cual se decidió presentar primero los estudios correspondientes a $\mathrm{H}_{2} \mathrm{Nep}$.

Todos los experimentos se realizaron a pH fisiológico debido a que, como se explicó en el Capítulo 2, estos dihidroderivados están presentes en los seres humanos. Asimismo, tienen valores de pKa mayores a 10, motivo por el cual, en soluciones neutras, solo está presente la forma ácida de estos compuestos. Se determinaron los rendimientos cuánticos de consumo del dihidroderivado a distintas concentraciones de $\mathrm{O}_{2}$ (condiciones de aerobiosis y anaerobiosis), se estudiaron los productos, la producción de EROs y se investigaron los mecanismos de reacción.

\subsection{Irradiación de $\mathrm{H}_{2} \mathrm{Nep}$}

Se irradiaron soluciones acuosas del dihidroderivado a pH fisiológico $(\sim 7,0)$, utilizando el Sistema de irradiación I (350 nm, Sección 4.3). Los experimentos se realizaron en aire, en atmósfera saturada en $\mathrm{O}_{2}$ y en condiciones anaeróbicas (Sección 4.2.4). En la Figura 8.1 se muestran los cambios espectrales sufridos por soluciones de $\mathrm{H}_{2} \mathrm{Nep}\left(\left[\mathrm{H}_{2} \mathrm{Nep}\right]=107 \mu \mathrm{M}, 124 \mu \mathrm{M}\right.$ y $148 \mu \mathrm{M}$ en atmósferas con porcentaje de $\mathrm{O}_{2}$ de $0 \%$, $21 \%$ y $100 \%$, respectivamente). En todos los casos se observaron cambios espectrales idénticos: disminución de la intensidad de la banda de menor energía del reactivo y aumento de la absorbancia en el intervalo 240 - $270 \mathrm{~nm}$. Dichos cambios espectrales ocurrieron a la misma velocidad en las 3 condiciones estudiadas. Más aún, se observaron varios puntos isosbésticos a 240, 268, 288 y 303 nm, los cuales fueron iguales para las tres condiciones y se mantuvieron constantes por más de 6 min de irradiación. Estos 
resultados sugieren que un único proceso fotoquímico tiene lugar cuando se irradian soluciones de $\mathrm{H}_{2} \mathrm{Nep}$ y que el mismo es independiente de la presencia de $\mathrm{O}_{2}$.

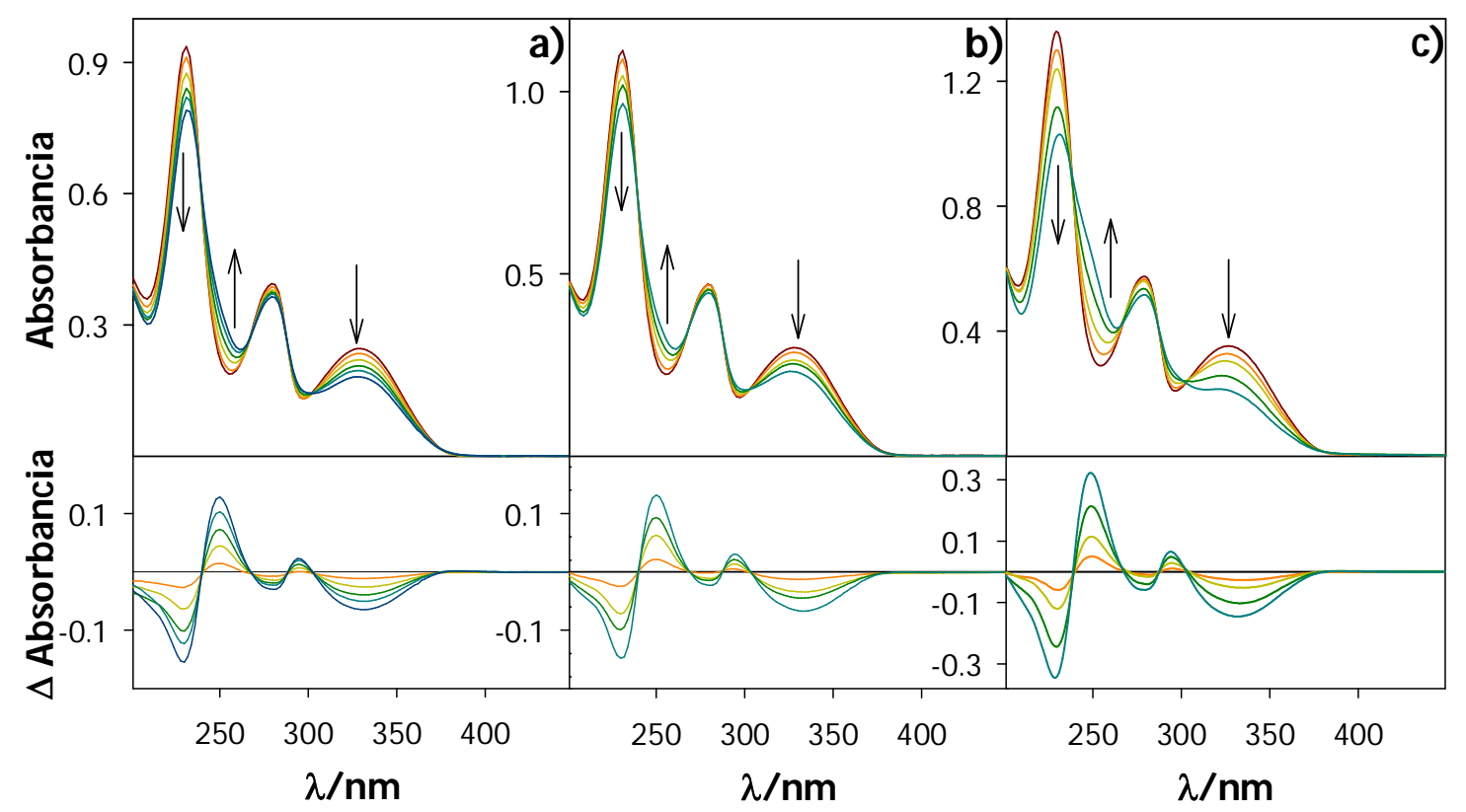

Figura 8.1 Evolución de los espectros de absorción durante la irradiación de soluciones acuosas de $\mathrm{H}_{2} \mathrm{Nep}$. a) $\left[\mathrm{H}_{2} \mathrm{Nep}\right]_{0}=107 \mu \mathrm{M}, \mathrm{pH}=6,7$, ti = 0, 1, 2, 3, 4, $5 \mathrm{~min}$, en condiciones anaeróbicas, b) $\left[\mathrm{H}_{2} \mathrm{Nep}\right]_{0}=124 \mu \mathrm{M}, \mathrm{pH}=7,0$, ti $=0,1,2,3,4,5 \mathrm{~min}$, en condiciones aeróbicas, c) $\left[\mathrm{H}_{2} \mathrm{Nep}\right]_{0}=148 \mu \mathrm{M}, \mathrm{pH}=6,8, \mathrm{ti}=0,1,2,4,6 \mathrm{~min}$, en atmósfera saturada en $\mathrm{O}_{2}$. Camino óptico $=0,4 \mathrm{~cm}$. Sistema de irradiación I. Parte inferior: EDE

Tal como se describió en el Capítulo 2, los 7,8-dihidroderivados no son completamente estables en soluciones acuosas aireadas, sufriendo una lenta oxidación. Debido a que todas las soluciones se prepararon en condiciones aeróbicas, previo al estudio de las reacciones fotoquímicas, las soluciones de $\mathrm{H}_{2} \mathrm{Nep}$ fueron analizadas por HPLC. Como se evidencia en la Figura 8.2, el primer cromatograma que corresponde al reactivo previa irradiación muestra el pico de $\mathrm{H}_{2} \mathrm{Nep}$ a un tiempo de retención ( $\operatorname{tr}$ ) de 6,0 min y dos pequeños picos a $\operatorname{tr}=9,0$ y 11,1 min que se encuentran como impurezas en las soluciones de $\mathrm{H}_{2} \mathrm{Nep}$, en muy pequeña proporción. El primero ( $\operatorname{tr}=9,0 \mathrm{~min}$ ) corresponde a un compuesto desconocido (llamado arbitrariamente compuesto X), del cual no se posee el correspondiente patrón. El mismo posee características espectrales similares a las dihidropterinas. Presenta máximos en 223, 275 y 313 nm, valores muy parecidos a los máximos de absorción que presenta el espectro de dihidroxantopterina ( $\mathrm{H}_{2}$ Xap, 223, 274 y $310 \mathrm{~nm}$ ). El segundo pico ( $\mathrm{tr}=11,1 \mathrm{~min}$ ) corresponde justamente a $\mathrm{H}_{2}$ Xap y se encuentra en una proporción de $\sim 1,2-1,5 \%$ con respecto a la concentración de $\mathrm{H}_{2} \mathrm{Nep}$. Como se detalló en el Capítulo 2, $\mathrm{H}_{2} \mathrm{Xap}$ es el producto principal de la reacción de oxidación de $\mathrm{H}_{2} \mathrm{Nep}$ por $\mathrm{O}_{2}$ disuelto, ${ }^{1}$ por lo tanto, su concentración se 
incrementa cuando se almacena la solución de $\mathrm{H}_{2} \mathrm{Nep}$ por un largo período de tiempo en contacto con $\mathrm{O}_{2}$. Sin embargo, a pesar de la presencia de estas impurezas es importante destacar que, por un lado, las mismas están en muy baja proporción $(<2 \%)$ y, por otro, estos dos contaminantes no son capaces de afectar la fotoquímica de $\mathrm{H}_{2} \mathrm{Nep}$, pues estos compuestos no presentan absorción en la región del espectro correspondiente a la longitud de onda de excitación (Figura 8.3).
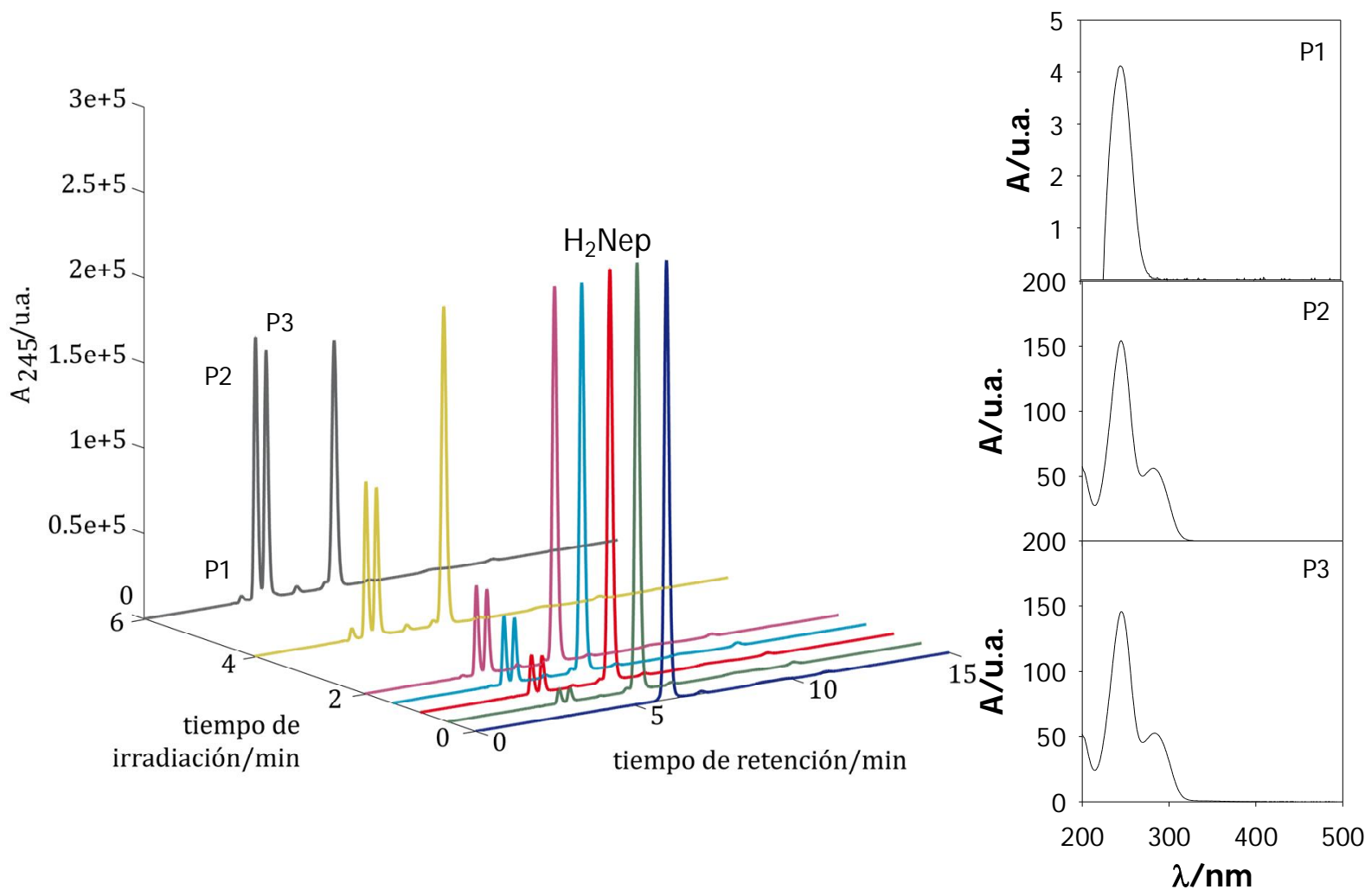

Figura 8.2 Cromatogramas $\left(\lambda_{\text {an }}=245 \mathrm{~nm}\right)$ obtenidos de la irradiación de una solución de $\mathrm{H}_{2} \mathrm{Nep}$ en presencia de $\mathrm{O}_{2}\left(\left[\mathrm{H}_{2} \mathrm{Nep}_{0}=148 \mu \mathrm{M}, \mathrm{pH}=6,6\right)\right.$. Espectros de absorción de P1, P2 y P3. Sistema de irradiación I y Equipo HPLC I.

Cuando se analizaron las soluciones irradiadas en presencia de $\mathrm{O}_{2}$ por HPLC, se observó que a medida que pasa el tiempo de irradiación el pico del reactivo disminuye (Figura 8.2), se mantienen relativamente constantes los picos de $\mathrm{H}_{2} \mathrm{Xap}$ y del compuesto $\mathrm{X}$ y, además, aparecen 3 productos (llamados arbitrariamente P1, P2 y P3). Estos 3 productos presentan tr menores al del reactivo. Examinando los cromatogramas a distintas longitudes de onda se observa que los picos P2 y P3 crecen simultáneamente con el tiempo de irradiación y son mucho mayores que el pico P1, sugiriendo que este último es un producto minoritario. Como se aprecia en la Figura 8.2, los espectros de absorción de P2 y P3 son muy parecidos entre sí, con máximos a 245 y $282 \mathrm{~nm}$. Esto es consistente con los cambios espectrales observados previamente, debido a que los 
productos aumentan la intensidad de la absorbancia en el intervalo de 240 - $270 \mathrm{~nm}$. Los mismos perfiles cromatográficos se observaron para las soluciones irradiadas en ausencia de $\mathrm{O}_{2} \mathrm{y}$ en atmósfera saturada en $\mathrm{O}_{2}$.

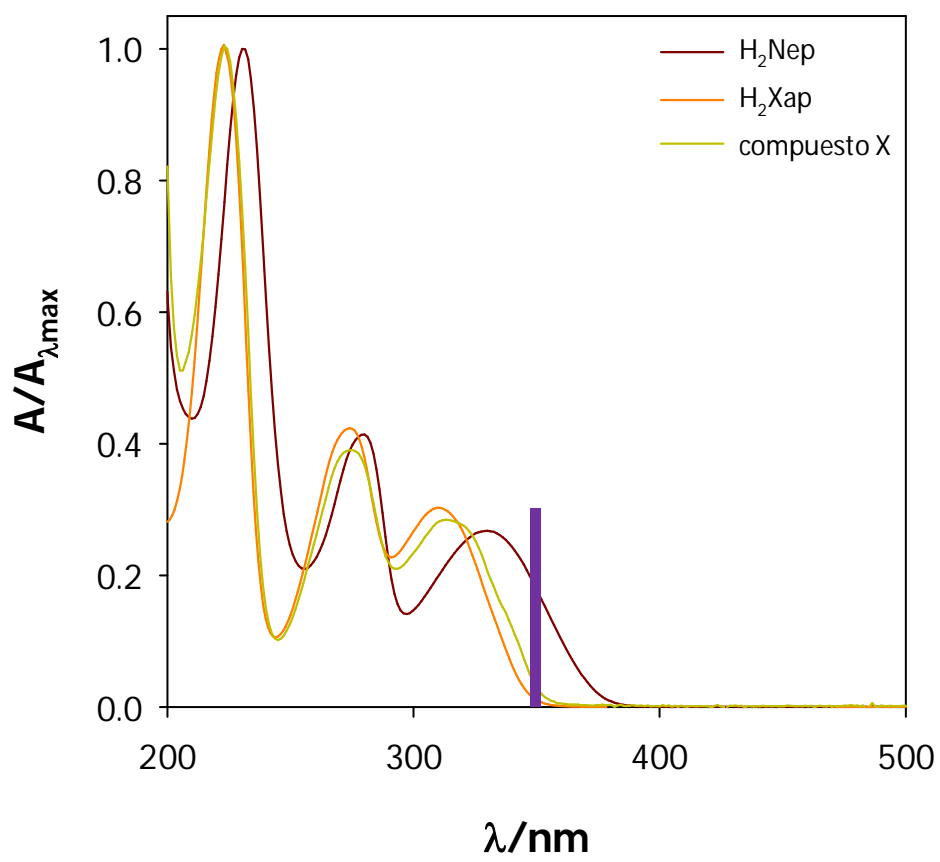

Figura 8.3 Espectros de absorción normalizados de $\mathrm{H}_{2} \mathrm{Nep}, \mathrm{H}_{2} \mathrm{Xap}$ y compuesto $\mathrm{X}$, registrados con el detector del Equipo HPLC I. La posición de la barra violeta señala la $\lambda_{\text {exc }}$ utilizada en el Sistema de irradiación I.

Luego, se realizaron los perfiles de concentración del reactivo en función del tiempo de irradiación (Figura 8.4), y en concordancia con el análisis espectral, en las tres condiciones experimentales, se aprecian perfiles muy parecidos. Los valores de velocidad inicial de consumo de $\mathrm{H}_{2} \mathrm{Nep}$ obtenidos a las distintas concentraciones de $\mathrm{O}_{2}$ fueron muy similares: $0,20 \pm 0,01 ; 0,20 \pm 0,01$ y 0,23 $\pm 0,01 \mu \mathrm{M} / \mathrm{s}$, para la irradiación en presencia de $\mathrm{O}_{2}$, en ausencia de $\mathrm{O}_{2}$ y en condiciones de saturación en $\mathrm{O}_{2}$, respectivamente.

En el caso de los productos P2 y P3, se graficaron los perfiles de área vs. tiempo de irradiación (Figura 8.4), considerando que fue imposible determinar su concentración debido a la falta de estándares o patrones para realizar las correspondientes curvas de calibración. Es notorio como las áreas de ambos productos evolucionan de manera similar. Asimismo, la velocidad de incremento de las áreas es igual, dentro del error experimental, para las tres condiciones, ratificando que el $\mathrm{O}_{2}$ no afecta la velocidad del proceso y que, indiscutiblemente, no se necesita dicho gas para generar los productos P2 y P3. Es importante destacar que el área del producto P1 tuvo un crecimiento despreciable en el tiempo que duraron los experimentos. 

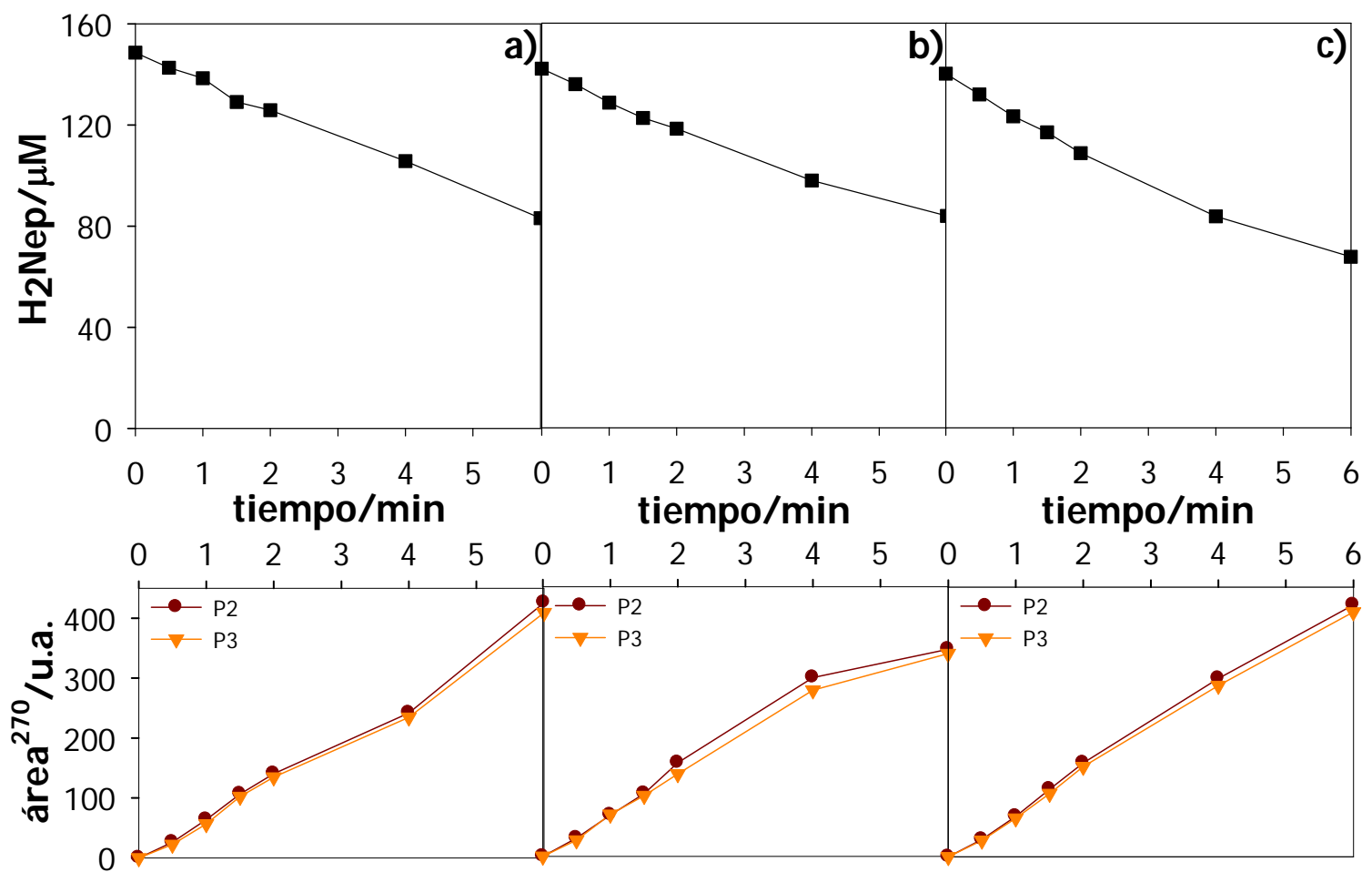

Figura 8.4 Evolución de la concentración de $\mathrm{H}_{2} \mathrm{Nep}$ con el tiempo de irradiación. $\left[\mathrm{H}_{2} \mathrm{Nep}_{0}\right.$ $=148 \mu \mathrm{M}, \mathrm{pH}=6,6$ : a) solución aireada, b) condiciones anaeróbicas y c) saturación en $\mathrm{O}_{2}$. Sistema de irradiación I y Equipo HPLC I. Parte inferior: evolución en el tiempo de las áreas de los picos cromatográficos correspondientes a P2 y P3.

En otro experimento, se determinó la variación en la concentración de $\mathrm{O}_{2}$ disuelto en la solución durante la irradiación de soluciones de $\mathrm{H}_{2} \mathrm{Nep}$. Para tal fin se utilizó un electrodo selectivo a dicho gas (Sección 5.7). Como se puede observar en la Figura 8.5, el consumo de $\mathrm{O}_{2}$ es muy bajo si se tiene en cuenta que la concentración de $\mathrm{H}_{2} \mathrm{Nep}$ varió de 141 a $30 \mu \mathrm{M}$ en ese período de tiempo. Es decir que si la relación de consumo de $\mathrm{O}_{2} \mathrm{y}$ $\mathrm{H}_{2} \mathrm{Nep}$ fuera 1 a 1 , entonces el consumo esperado de $\mathrm{O}_{2}$ sería de $111 \mu \mathrm{M}$. Sin embargo, los resultados experimentales muestran que, en las condiciones de irradiación empleadas, la cantidad total de $\mathrm{O}_{2}$ consumida fue de sólo $33 \mu \mathrm{M}$. Esto evidencia claramente que en la formación de P1, P2 y P3 no participa el $\mathrm{O}_{2}$ disuelto en el medio. La variación en la concentración de $\mathrm{O}_{2}$ puede atribuirse a la oxidación de $\mathrm{H}_{2} \mathrm{Nep}$ descripta en la Sección 2.3, a una ligera disminución de la solubilidad por aumento de la temperatura o a una combinación de ambas. 


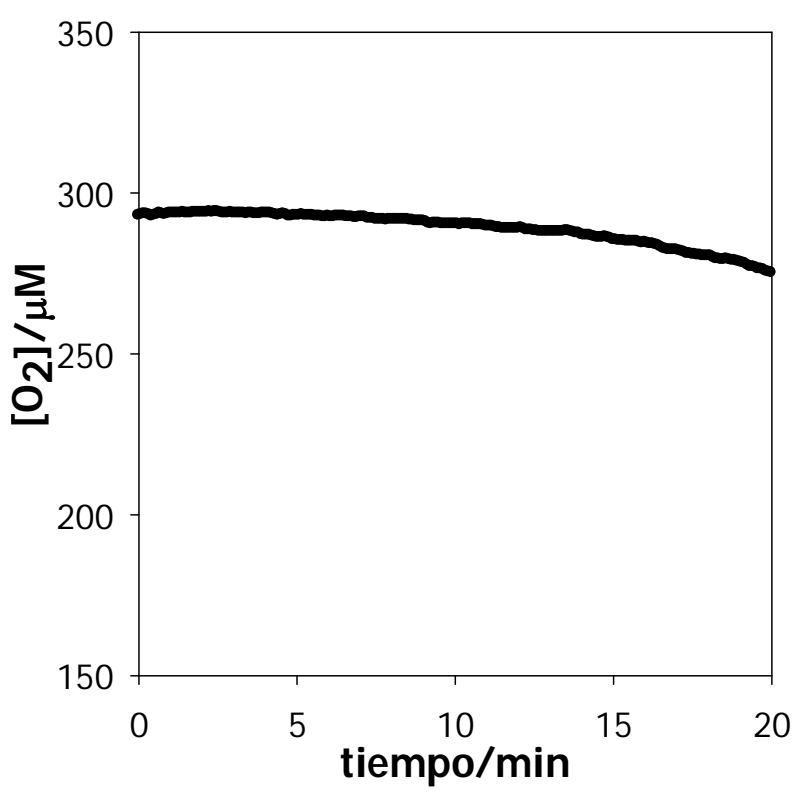

Figura 8.5 Evolución de la concentración de $\mathrm{O}_{2}$ en soluciones de $\mathrm{H}_{2} \mathrm{Nep}$ con el tiempo de irradiación. $\left[\mathrm{H}_{2} \mathrm{Nep}_{0}=141 \mu \mathrm{M}, \mathrm{pH}=7,2\right.$. Sistema de irradiación $\mathrm{I}$.

\subsection{Análisis de los productos por espectrometría de masa}

De lo expuesto anteriormente se desprende que las características espectrales de los productos principales P2 y P3 (Figura 8.2) no son compatibles con los espectros de las dihidropterinas o con los de las pterinas oxidadas. En consecuencia, estos productos no pudieron ser identificados cromatográficamente por comparación con patrones comerciales disponibles en el laboratorio. Por lo tanto, y con el objeto de realizar una primera caracterización de P2 y P3, se recurrió al uso de técnicas de espectrometría de masa.

Las soluciones de $\mathrm{H}_{2} \mathrm{Nep}$ fueron irradiadas con el Sistema de irradiación II (335 $\mathrm{nm}$, Sección 4.3) hasta obtener una cantidad apreciable de productos (ti = $30 \mathrm{~min}$ ). Luego, se analizaron las muestras por espectrometría de masa con ionización electrospray (Equipo MS I), previa separación de los productos por HPLC (Sección 5.4). El análisis fue llevado a cabo en modo positivo y negativo (ESI+ y ESI- ${ }^{-}$respectivamente).

Primero, se analizó la muestra sin irradiar, donde se detectó el ión molecular del reactivo $\mathrm{H}_{2} \mathrm{Nep}$ como $[\mathrm{M}-\mathrm{H}]-{ }^{-}$a $\mathrm{m} / \mathrm{z}=254,2$ (modo $\mathrm{ESI}-$ ). En modo $\mathrm{ESI}^{+}$, se detectó como $[\mathrm{M}+\mathrm{H}]^{+}$y su aducto $[\mathrm{M}+\mathrm{Na}]^{+}$con valores de $\mathrm{m} / \mathrm{z}=256,2$ y 278,2, respectivamente (Figura 8.6.a). La investigación se continuó utilizando el modo $\mathrm{ESI}^{+}$, porque en estas condiciones de análisis se obtuvo una mejor relación señal/ruido. En las muestras irradiadas se verificó el pico del reactivo y se obtuvo el ión molecular de los dos productos, $\mathrm{P} 2$ y $\mathrm{P} 3$, con $\mathrm{m} / \mathrm{z}=511$. Este valor corresponde a $\left[\mathrm{M}_{2}+\mathrm{H}\right]+=2$ x $255+1$ y se apreció también un pico en $\mathrm{m} / \mathrm{z}=534,4$ correspondiente al aducto [ $\left.\mathrm{M}_{2}+\mathrm{Na}\right]^{+}$. Además, se 
detectó la presencia de un pico significativo en $\mathrm{m} / \mathrm{z}=256,2$ (igual al del reactivo). Esto se observa claramente en la Figura 8.6.b donde se expone, a modo de ejemplo, el espectro de masa de P2. El espectro de masa correspondiente a P3 es igual al de P2. Consecuentemente, las masas moleculares de P2 y P3 son equivalentes a exactamente el doble de la masa molecular de $\mathrm{H}_{2} \mathrm{Nep}$. Estos resultados sugieren fuertemente que P2 y P3 son dímeros del reactivo y que la vía de fragmentación más eficiente durante el proceso de electrospray es la rotura de estos dímeros para generar el correspondiente monómero, motivo por el cual se observa el pico en $\mathrm{m} / \mathrm{z}=256$ con mayor intensidad que el correspondiente ión molecular.

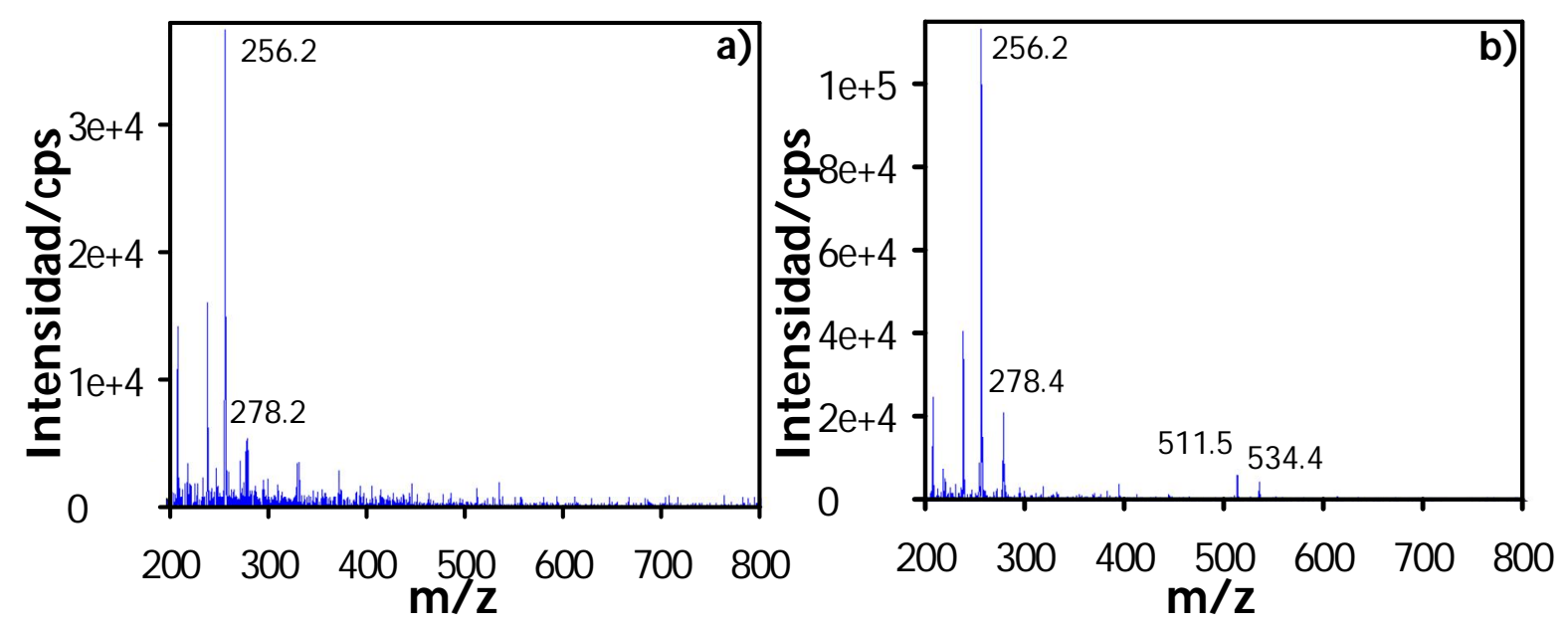

Figura 8.6 Espectros de masa (modo ESI+). a) Pico correspondiente a $\mathrm{H}_{2} \mathrm{Nep}$ en un cromatograma de la solución patrón. b) Pico correspondiente a P2 en un cromatograma de una solución de $\mathrm{H}_{2} \mathrm{Nep}$ irradiada en condiciones anaeróbicas, ti = $30 \mathrm{~min}$. Sistema de irradiación II y Equipo MS I.

Para corroborar los pesos moleculares, se realizaron experimentos utilizando un espectrómetro de masa de alta resolución con analizador TOF (Equipo MS II, Sección 5.4). Este equipo, de mayor sensibilidad, provee una mayor resolución en el análisis y proporciona valores $\mathrm{m} / \mathrm{z}$ de los distintos compuestos con hasta 4 decimales. Para el pico del cromatograma correspondiente a $\mathrm{H}_{2} \mathrm{Nep}$ se registró el ión molecular correspondiente a $[\mathrm{M}+\mathrm{H}]^{+}$con un valor de $\mathrm{m} / \mathrm{z}=256,2732$. Esto corresponde a una composición elemental de $\mathrm{C}_{9} \mathrm{H}_{13} \mathrm{~N}_{5} \mathrm{O}_{4}+1 \mathrm{H}$ con un error con respecto al valor teórico de 168,6 mDa. Al analizar luego la muestra de $\mathrm{H}_{2} \mathrm{Nep}$ irradiada, se procedió a calibrar el equipo con el valor teórico de la masa del reactivo previamente calculada $\mathrm{y}$, posteriormente, se determinaron los valores $\mathrm{m} / \mathrm{z}$ para los picos correspondientes a los dímeros. Para ambos productos P2 y P3 se observó el mismo ión molecular con valor de $\mathrm{m} / \mathrm{z}=511,2026$. Este valor corresponde a $\left[\mathrm{M}_{2}+\mathrm{H}\right]^{+}$con composición elemental de 
$\mathrm{C}_{18} \mathrm{H}_{26} \mathrm{~N}_{10} \mathrm{O}_{8}+1 \mathrm{H}$ y un error con el valor teórico de -1,3 mDa. En la Figura 8.7 se muestra el espectro de masa correspondiente al pico P2. Estos resultados confirman fehacientemente la naturaleza dimérica de los productos, los cuales están formados por dos moléculas de reactivo unidas entre sí y, cuya ionización da lugar a la formación y detección del monómero. Las estructuras de estos compuestos serán estudiadas más profundamente en el Capítulo 11.

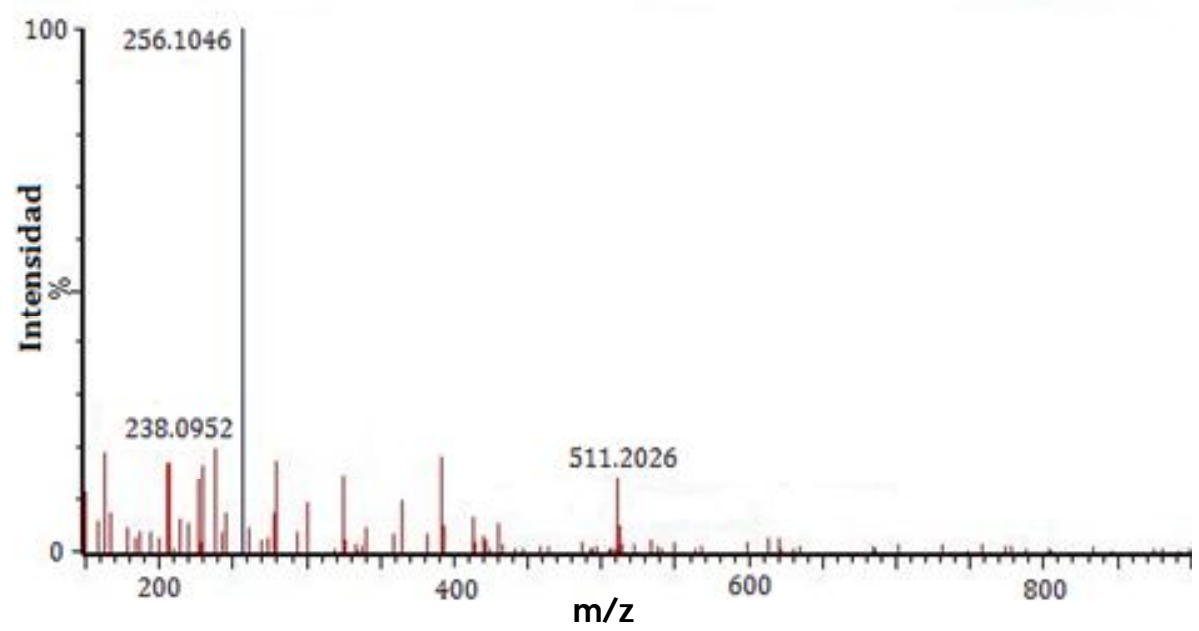

Figura 8.7 Espectros de masa (modo ESI+) de P2 de una solución de $\mathrm{H}_{2} \mathrm{Nep}$ irradiada en ausencia de $\mathrm{O}_{2}$ (ti $\left.=30 \mathrm{~min}\right)$. Sistema de irradiación II y Equipo MS II.

\subsection{Rendimientos cuánticos}

Los rendimientos cuánticos de consumo de reactivo ( $\Phi$-H2Nep) (Sección 4.4) fueron determinados en las distintas atmósferas (aire, saturación en $\mathrm{O}_{2}$ y condiciones anaeróbicas). El valor encontrado fue equivalente, dentro del error experimental, en las tres condiciones. De esta manera, se confirma que la eficiencia de fotodimerización de $\mathrm{H}_{2} \mathrm{Nep}$ es independiente de la concentración de $\mathrm{O}_{2}$. Por otro lado, estos resultados sugieren que la generación de los productos provendría, a diferencia de lo observado para las pterinas oxidadas, del estado excitado singlete de $\mathrm{H}_{2} \mathrm{Nep}\left({ }^{1} \mathrm{H}_{2} \mathrm{Nep} *\right)$ pues, en caso de proceder del estado excitado triplete $\left({ }^{3} \mathrm{H}_{2} \mathrm{Nep} *\right)$, $\Phi$-н2Nep se vería modificado con la concentración de $\mathrm{O}_{2}$. Esto concuerda con trabajos previos en los que se sugirió que los dihidroderivados no son capaces de generar ${ }^{1} \mathrm{O}_{2}$ debido a que no forman estados excitados tripletes. ${ }^{2}$

Dado que el proceso fotoquímico global es una fotodimerización, el mecanismo necesariamente debe involucrar alguna etapa bimolecular en la que participan dos moléculas de reactivo. Para investigar si este hecho implica una dependencia de la eficiencia del proceso con la concentración del reactivo, se determinaron los $\Phi$-H2Nep a 
diferentes valores de concentración inicial de reactivo. Se utilizó un intervalo de concentraciones de 1 a $160 \mu \mathrm{M}$. Es importante aclarar que no fue posible extender el rango a mayores valores debido a problemas de solubilidad del reactivo, ni a menores concentraciones debido a los límites de detección del cromatógrafo. Como se puede observar en la Figura 8.8, en el intervalo de concentraciones utilizado, Ф-H2Nep es independiente de la concentración inicial de $\mathrm{H}_{2} \mathrm{Nep}$ y el valor promedio obtenido es de $0,038 \pm 0,005$. Este valor es bajo si se compara con los valores obtenidos para Bip o Nep en ausencia de $\mathrm{O}_{2}\left(\Phi\right.$-Bip $=0,10 \pm 0,01$ y $\Phi_{\text {-Nep }}=0,11 \pm 0,01$; Capítulo 6$)$, pero es más elevado que los valores encontrados para otros dihidroderivados como ser 6-formil-7,8dihidropterina $\left(\mathrm{H}_{2} \mathrm{Fop}, \Phi_{\text {-н2Fop }}=0,00113 \pm 0,00009\right){ }^{3}$ No fue posible calcular los rendimientos cuánticos de los productos, debido a que no se poseen los estándares para realizar las correspondientes curvas de calibración.

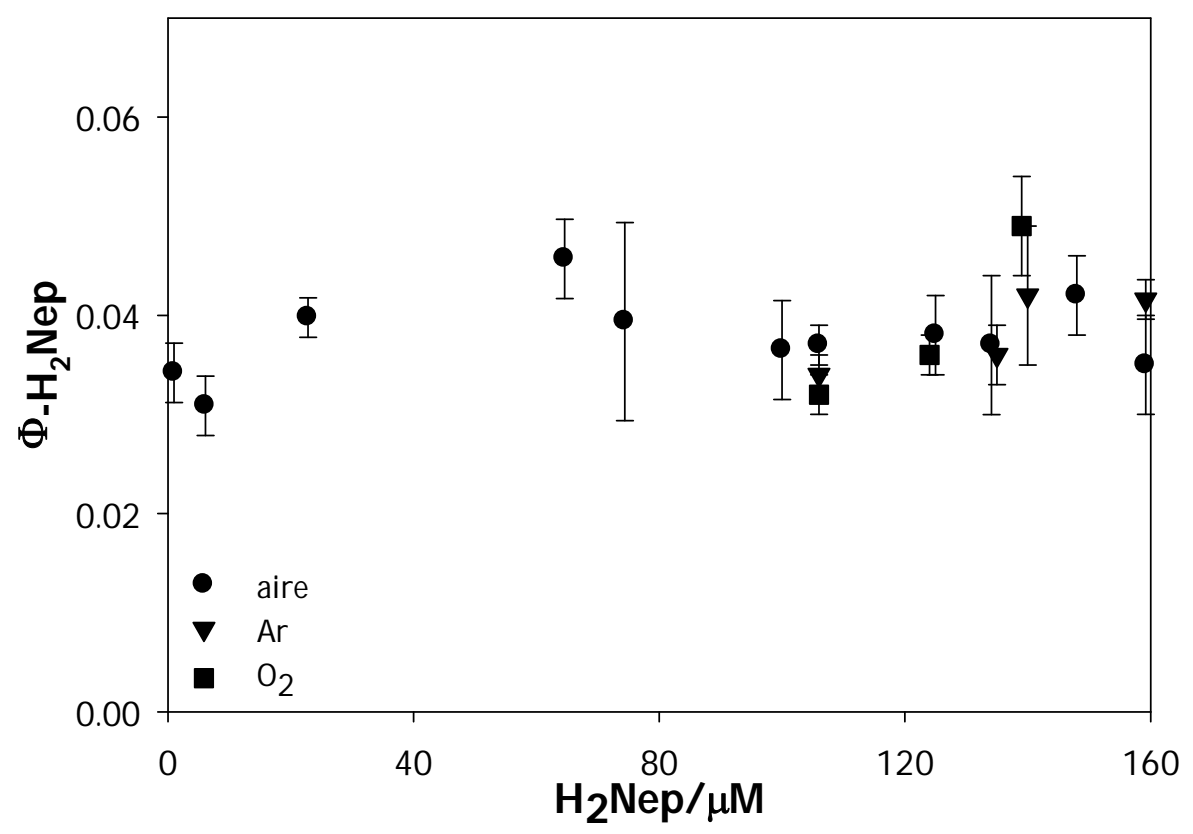

Figura 8.8 Rendimientos cuánticos de consumo de $\mathrm{H}_{2} \mathrm{Nep}(\Phi$-H2Nep) en función de la concentración de $\mathrm{H}_{2} \mathrm{Nep}$. Valores determinados a partir de soluciones saturadas en $\mathrm{Ar}$, aire y $\mathbf{O}_{2}$.

\subsection{Análisis del mecanismo de reacción}

Teniendo en cuenta que $\mathrm{H}_{2} \mathrm{Nep}$ no genera ${ }^{1} \mathrm{O}_{2} 2$ y que el rendimiento cuántico de consumo es independiente de la concentración de $\mathrm{O}_{2}$ disuelto en el medio, se puede descartar la participación del estado excitado triplete $\left({ }^{3} \mathrm{H}_{2} \mathrm{Nep} *\right)$ en el mecanismo de reacción. Por lo tanto, la fotodimerización podría consistir en un proceso bimolecular en 
el cual el estado excitado singlete de $\mathrm{H}_{2} \mathrm{Nep}\left({ }^{1} \mathrm{H}_{2} \mathrm{Nep} *\right)$ reacciona con otra molécula de $\mathrm{H}_{2} \mathrm{Nep}$ en estado fundamental (reacciones 1-3):

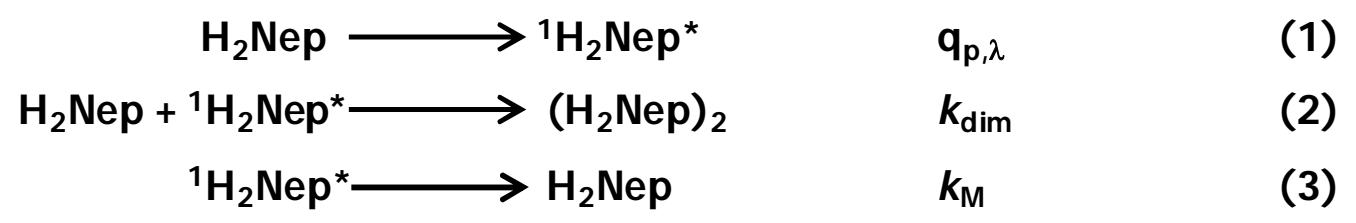

donde $\mathrm{q}_{\mathrm{p}, \lambda}$ es la intensidad de la radiación absorbida por $\mathrm{H}_{2} \mathrm{Nep}$ (einstein $\mathrm{L}^{-1} \mathrm{~s}^{-1}$ ); $\mathrm{k}_{\mathrm{dim}}$ es la constante de velocidad bimolecular de la reacción de dimerización $\left(\mathrm{M}^{-1} \mathrm{~S}^{-1}\right) ; \mathrm{k}_{\mathrm{M}}$ es la constante de velocidad monomolecular de desactivación de ${ }^{1} \mathrm{H}_{2} \mathrm{Nep}^{*}\left(\mathrm{~s}^{-1}\right)$ y comprende a $\mathrm{k}_{\mathrm{ISC}}$ (constante de entrecruzamiento de sistemas), $\mathrm{k}_{\mathrm{F}}$ (constante de fluorescencia) y $\mathrm{k}_{\mathrm{IC}}$ (constante de conversión interna). Como $\mathrm{k}_{\mathrm{ISC}} \mathrm{y} \mathrm{k}_{\mathrm{F}}$ son despreciables, $\mathrm{k}_{\mathrm{M}} \approx \mathrm{k}_{\mathrm{IC}}$.

Si este mecanismo fuese correcto, la velocidad de consumo de $\mathrm{H}_{2} \mathrm{Nep}$ estaría dada por la ecuación 4:

- $\left(\frac{\mathrm{d}\left[\mathrm{H}_{2} \mathrm{Nep}\right]}{\mathrm{dt}}\right)=2 \mathrm{k}_{\mathrm{dim}}\left[{ }^{1} \mathrm{H}_{2} \mathrm{Nep}^{*}\right]\left[\mathrm{H}_{2} \mathrm{Nep}\right]$

Suponiendo la hipótesis de estado estacionario (EE) para ${ }^{1} \mathrm{H}_{2} \mathrm{Nep}^{*}$, la concentración de dicha especie ([1 $\left.\left.{ }^{\mathrm{H}_{2} \mathrm{Nep} *}\right]_{\mathrm{EE}}\right)$ puede ser calculada de la siguiente manera:

$$
\begin{aligned}
& -\left(\frac{d\left[{ }^{1} \mathrm{H}_{2} \mathrm{Nep}^{*}\right]}{\mathrm{dt}}\right)=0=\mathrm{q}_{\mathrm{p}, \lambda}-\left(\mathrm{k}_{\mathrm{M}}\left[{ }^{1} \mathrm{H}_{2} \mathrm{Nep}^{*}\right]+\mathrm{k}_{\mathrm{dim}}\left[{ }^{1} \mathrm{H}_{2} \mathrm{Nep}^{*}\right]_{\mathrm{EE}}\left[\mathrm{H}_{2} \mathrm{Nep}\right]\right) \\
& \left(\mathrm{k}_{\mathrm{M}}+\mathrm{k}_{\mathrm{dim}}\left[\mathrm{H}_{2} \mathrm{Nep}\right]\right)\left[{ }^{1} \mathrm{H}_{2} \mathrm{Nep}^{*}\right]_{\mathrm{EE}}=\mathrm{q}_{\mathrm{p}, \lambda} \\
& {\left[{ }^{1} \mathrm{H}_{2} \mathrm{Nep}^{*}\right]_{\mathrm{EE}}=\frac{\mathrm{q}_{\mathrm{p}, \lambda}}{\mathrm{k}_{\mathrm{M}}+\mathrm{k}_{\mathrm{dim}}\left[\mathrm{H}_{2} \mathrm{Nep}\right]}}
\end{aligned}
$$

Reemplazando [ ${ }^{1} \mathrm{H}_{2} \mathrm{Nep} *$ en la ecuación 4, se obtiene:

$$
\text { - }\left(\frac{d\left[H_{2} N e p\right]}{d t}\right)=\frac{2 q_{p, \lambda} k_{d i m}\left[H_{2} N e p\right]}{k_{M}+k_{\text {dim }}\left[H_{2} N e p\right]}
$$

Consecuentemente, el rendimiento cuántico de consumo de reactivo (Sección 4.4) está dado por:

$$
\Phi_{-\mathrm{H} 2 \mathrm{Nep}}=\frac{2 \mathrm{k}_{\mathrm{dim}}\left[\mathrm{H}_{2} \mathrm{Nep}\right]}{\mathrm{k}_{\mathrm{M}}+\mathrm{k}_{\mathrm{dim}}\left[\mathrm{H}_{2} \mathrm{Nep}\right]}
$$


La expresión obtenida para $\Phi_{\text {-H2Nep }}$ muestra una dependencia de este parámetro con $\left[\mathrm{H}_{2} \mathrm{Nep}\right]$. Esto se contradice con los resultados experimentales ya expuestos, tales como, la cinética de pseudoprimer orden hasta un $40 \%$ de consumo del reactivo (Figura 8.4) y el $\Phi$-H2Nep independiente de $\left[\mathrm{H}_{2} \mathrm{Nep}\right](\cong 0,04)$.

Otro mecanismo posible sería que ${ }^{1} \mathrm{H}_{2} \mathrm{Nep} *$ pueda convertirse en un intermediario (IS) que reacciona con $\mathrm{H}_{2} \mathrm{Nep}$ en estado basal para generar los dímeros (reacciones 10-13):

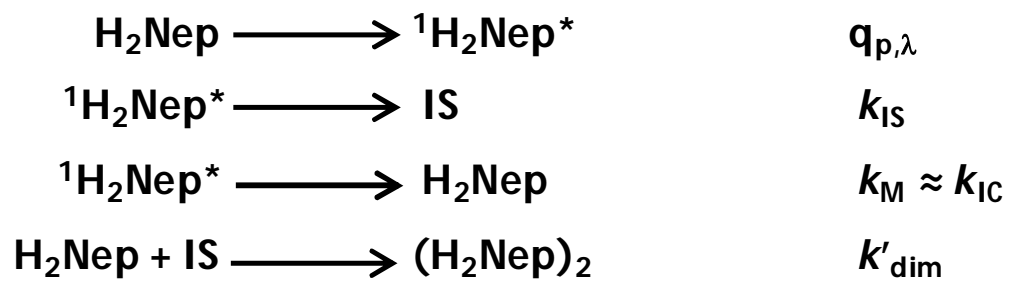

donde $\mathrm{k}_{\mathrm{IS}}$ es la constante de velocidad monomolecular de formación de IS y k'dim es la constante de velocidad bimolecular de la reacción de dimerización. En este caso la velocidad de consumo del reactivo estaría dada por:

$$
-\left(\frac{d\left[H_{2} N e p\right]}{d t}\right)=2 k_{d i m}^{\prime}[I S]\left[H_{2} N e p\right]
$$

Asumiendo condiciones de estado estacionario (EE) para el caso del intermediario, la concentración del mismo ([IS $]_{\mathrm{EE}}$ ) puede calcularse como:

$$
\begin{aligned}
& \left(\frac{d[I S]}{d t}\right)=0=k_{I S}\left[{ }^{1} H_{2} N^{*}{ }^{*}\right]-k_{d i m}^{\prime}[I S]_{\mathrm{EE}}\left[H_{2} \mathrm{Nep}\right] \\
& k_{\mathrm{IS}}\left[{ }^{1} \mathrm{H}_{2} \mathrm{Nep}^{*}\right]=\mathrm{k}_{\mathrm{dim}}^{\prime}[\mathrm{IS}]_{\mathrm{EE}}\left[\mathrm{H}_{2} \mathrm{Nep}\right] \\
& \frac{\mathrm{k}_{\mathrm{II}}\left[{ }^{1} \mathrm{H}_{2} \mathrm{Nep}^{*}\right]}{\mathrm{k}_{\mathrm{dim}}^{\prime}\left[\mathrm{H}_{2} \mathrm{Nep}\right]}=[\mathrm{IS}]_{\mathrm{EE}}
\end{aligned}
$$

Y para el estado excitado singlete $\left[{ }^{1} \mathrm{H}_{2} \mathrm{Nep} *\right]_{\mathrm{EE}}$

$$
\begin{aligned}
& \left(\frac{d\left[{ }^{1} \mathrm{H}_{2} \mathbf{N e p}^{*}\right]}{\mathrm{dt}}\right)=0=q_{\mathrm{p}, \lambda}-\left(\mathrm{k}_{\mathrm{IC}}\left[{ }^{1} \mathrm{H}_{2} \mathrm{Nep}^{*}\right]_{\mathrm{EE}}+\mathrm{k}_{\mathrm{IS}}\left[{ }^{1} \mathrm{H}_{2} \mathrm{Nep}^{*}\right]_{\mathrm{EE}}\right) \\
& \left(\mathrm{k}_{\mathrm{IC}}+\mathrm{k}_{\mathrm{IS}}\right)\left[{ }^{1} \mathrm{H}_{2} \mathrm{Nep}^{*}\right]_{\mathrm{EE}}=\mathrm{q}_{\mathrm{p}, \lambda}
\end{aligned}
$$




$$
\left[{ }^{1} \mathbf{H}_{2} \operatorname{Nep}^{*}\right]_{\mathrm{EE}}=\frac{\mathbf{q}_{\mathrm{p}, \lambda}}{\mathbf{k}_{\mathrm{IC}}+\mathbf{k}_{\mathrm{IS}}}
$$

Reemplazando $\left[{ }^{1} \mathrm{H}_{2} \mathrm{Nep} *\right]_{\mathrm{EE}}$ en la ecuación (17) y luego [IS $]_{\mathrm{EE}}$ en (14), se obtiene:

$$
\begin{gathered}
{[\mathrm{IS}]_{\mathrm{EE}}=\frac{k_{\mathrm{IS}} q_{\mathrm{p}, \lambda}}{k_{\mathrm{dim}}^{\prime}\left[\mathrm{H}_{2} \operatorname{Nep}\right]\left(k_{\mathrm{IC}}+k_{\mathrm{IS}}\right)}} \\
-\left(\frac{d\left[\mathrm{H}_{2} \mathrm{Nep}\right]}{\mathrm{dt}}\right)=\frac{2 k_{\mathrm{IS}} \mathbf{q}_{\mathrm{p}, \lambda}}{k_{\mathrm{IC}}+\mathbf{k}_{\mathrm{IS}}}
\end{gathered}
$$

Y el rendimiento cuántico queda expresado por la siguiente ecuación:

$$
\Phi_{-\mathrm{H} 2 \mathrm{Nep}}=\frac{2 \mathrm{k}_{\mathrm{IS}}}{\mathrm{k}_{\mathrm{IC}}+\mathrm{k}_{\mathrm{IS}}}
$$

En este caso, $\Phi$-H2Nep debería ser constante independientemente de la concentración de $\mathrm{H}_{2} \mathrm{Nep}$ inicial, tal como se observa experimentalmente (Figura 8.7). Teniendo en cuenta que el valor calculado de $\Phi$-H2Nep es cercano a 0,04, la constante de velocidad de fotodimerización/ fototautomerización de $\mathrm{H}_{2} \mathrm{Nep}\left(\mathrm{k}_{\mathrm{Is}}\right.$, reacción (11) podría estimarse en $2 \%$ de la constante de velocidad de desactivación de $\mathrm{S}_{1}\left(\mathrm{k}_{\mathrm{IS}} / \mathrm{k}_{\mathrm{IC}} \approx 0,02\right)$.

\subsection{Conclusiones}

A lo largo de este capítulo se ha demostrado que durante la irradiación de soluciones acuosas de $\mathrm{H}_{2} \mathrm{Nep}$, ya sea en presencia o ausencia de $\mathrm{O}_{2}$, se produce una fotodimerización con rendimiento cuántico de consumo de reactivo ( $\Phi_{\text {-H2Nep }}$ ) de 0,038 \pm 0,005. A partir del estado excitado singlete del reactivo $\left({ }^{1} \mathrm{H}_{2} \mathrm{Nep} *\right)$, se genera un intermediario (IS) que luego reacciona con una molécula de $\mathrm{H}_{2} \mathrm{Nep}$ en estado fundamental, dando lugar a la formación de dímeros con un peso molecular exactamente

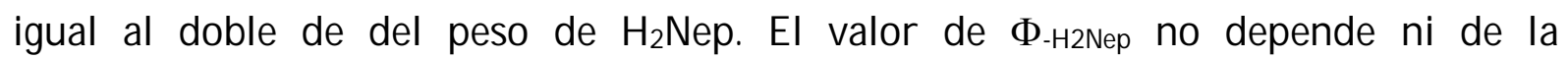
concentración de $\mathrm{O}_{2}$ ni de la concentración inicial de reactivo. El análisis global de los resultados permite plantear el mecanismo de reacción presentado en el Esquema 8.1. 


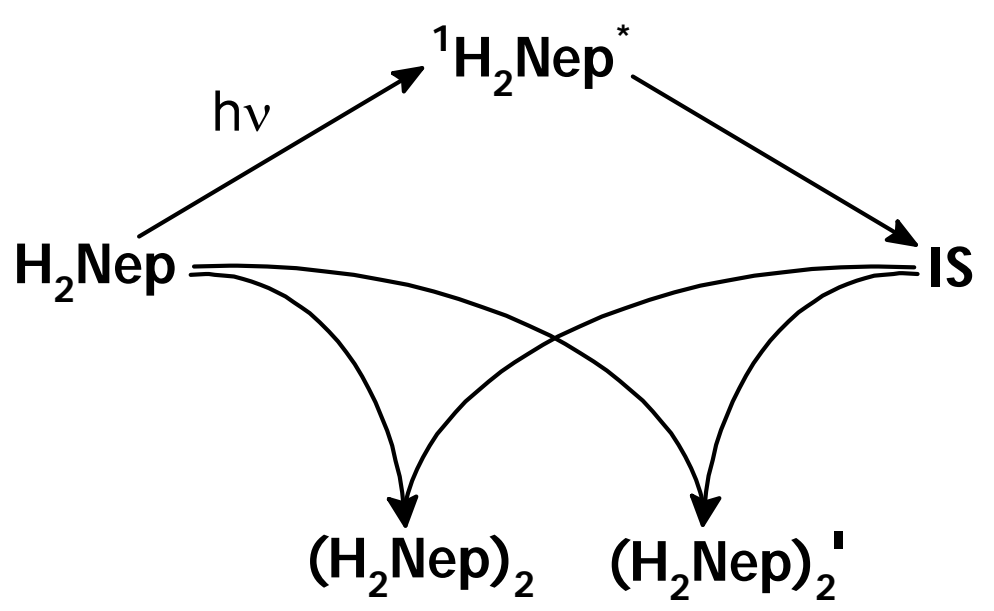

Esquema 8.1 Mecanismo de fotodimerización $\mathrm{H}_{2} \mathrm{Nep}$ bajo irradiación UV-A en solución acuosa.

\subsection{Referencias}

1. M. L. Dántola, M. Vignoni, A. L. Capparelli, C. Lorente and A. H. Thomas, Stability of 7,8-dihydropterins in air-equilibrated aqueous solutions, Helvetica Chimica Acta, 2008, 91, 411-425.

2. M. L. Dántola, A. H. Thomas, A. M. Braun, E. Oliveros and C. Lorente, Singlet oxygen $\left(\mathrm{O}_{2}\left({ }^{1} \Delta_{\mathrm{g}}\right)\right)$ quenching by dihydropterins, Journal of Physical Chemistry A, 2007, 111, 4280-4288.

3. M. L. Dántola, A. H. Thomas, E. Oliveros and C. Lorente, Visible-light photochemistry of 6-formyl-7,8dihydropterin in aqueous solution, Journal of Photochemistry and Photobiology A: Chemistry, 2010, 209, 104-110. 
Capítulo 8 


\section{Capítulo 9}

\section{Fotoquímica de 7,8-dihidrobiopterina}

La fotoquímica de 7,8-dihidrobiopterina ( $\left.\mathrm{H}_{2} \mathrm{Bip}\right)$ en solución acuosa se presenta en un capítulo separado al correspondiente a la fotoquímica de 7,8-dihidroneopterina ( $\mathrm{H}_{2} \mathrm{Nep}$ ) porque, como se mencionó anteriormente, el comportamiento fotoquímico de ambos compuestos es diferente. Para facilitar la lectura de este capítulo se puede adelantar que, además de fotodimerizar, $\mathrm{H}_{2}$ Bip sufre, en presencia de $\mathrm{O}_{2}$, un proceso de fotooxidación. Esta fotooxidación presenta una cinética compleja y de difícil interpretación, motivo por el cual la descripción de su mecanismo de reacción se analizará en detalle recién en el próximo capítulo. En el presente se muestran los resultados correspondientes a la identificación de fotoproductos, determinación de rendimientos cuánticos de consumo de $\mathrm{H}_{2}$ Bip e investigación de la participación del $\mathrm{O}_{2}$. Finalmente se discuten las implicancias biológicas de los resultados presentados.

\subsection{Irradiación en ausencia de oxígeno}

Soluciones acuosas de $\mathrm{H}_{2} \mathrm{Bip}(50-200 \mu \mathrm{M}$, a $\mathrm{pH}=7,0$ ), fueron saturadas en $\mathrm{Ar}$, para eliminar el $\mathrm{O}_{2}$ disuelto (Sección 4.2.4). Luego fueron irradiadas utilizando los dos sistemas de irradiación previamente descriptos (Sección 4.3). Esta irradiación produjo cambios característicos en el espectro de absorción muy similares a los observados para $\mathrm{H}_{2}$ Nep: disminuyó la banda centrada a $330 \mathrm{~nm}$ (Figura 9.1) y aumentó la absorbancia a longitudes de onda menores a $300 \mathrm{~nm}$. Además, se observaron puntos isosbésticos a 239, 270, 286 y $304 \mathrm{~nm}$ que permanecieron invariables por más de $10 \mathrm{~min}$ de irradiación, sugiriendo la presencia de un único proceso químico durante el intervalo de tiempo analizado. 


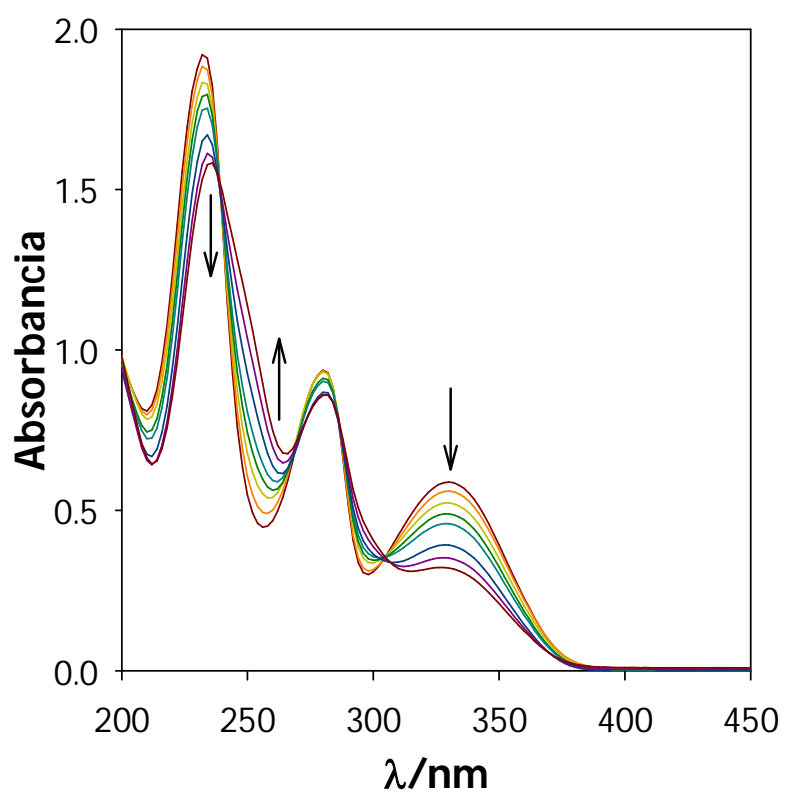

Figura 9.1 Evolución del espectro de absorción de una solución de $\mathrm{H}_{2} B i p$ en ausencia de $\mathrm{O}_{2}\left(\left[\mathrm{H}_{2} \mathrm{Bip}_{0}=108 \mu \mathrm{M}, \mathrm{pH}=6,6\right)\right.$ en función del tiempo de irradiación. Espectros registrados a $0,1,2,3,4,5,6,8$ y $10 \mathrm{~min}$. Camino óptico $=1 \mathrm{~cm}$. Sistema de irradiación $\mathrm{I}$.

Las muestras irradiadas se analizaron por HPLC y se observó un comportamiento similar al descripto para $\mathrm{H}_{2} \mathrm{Nep}$. Por un lado, el área del pico de $\mathrm{H}_{2}$ Bip disminuyó con el tiempo de fotólisis. A su vez, se observó la aparición de tres picos (P1, P2 y P3), con tiempos de retención menores al del reactivo, cuyas áreas se incrementaron a medida que aumentó el tiempo de irradiación (Figura 9.2). Asimismo, el análisis de los cromatogramas a distintas longitudes de onda mostró que el área de los picos P2 y P3 aumenta simultáneamente, y a su vez, son mucho mayores que el área de P1, lo cual sugiere que P1 es un producto minoritario. Mediante el uso del detector UV-vis con arreglo de diodos del Equipo HPLC I se obtuvieron los espectros de absorción de estos tres productos. Claramente, P2 y P3 muestran espectros de absorción muy similares entre sí, con una banda con máximos en 246 y 282 nm y sin absorción por arriba de 320 nm (Figura 9.2). Estos resultados son consistentes con los espectros de absorción mostrados en la Figura 9.1. Apreciando la similitud con los productos de la fotólisis de $\mathrm{H}_{2} \mathrm{Nep}$ (Capítulo 8), ya sea en tiempos de retención como en espectros de absorción, se puede pensar en la formación de dímeros de $\mathrm{H}_{2}$ Bip. Esta hipótesis será desarrollada con mayor detalle más adelante. Es importante mencionar además que, en las soluciones irradiadas en atmósfera de argón, no se manifestó la presencia de biopterina (Bip) ni de $\mathrm{H}_{2} \mathrm{O}_{2}$ en el análisis por HPLCy por el método enzimático, respectivamente. 

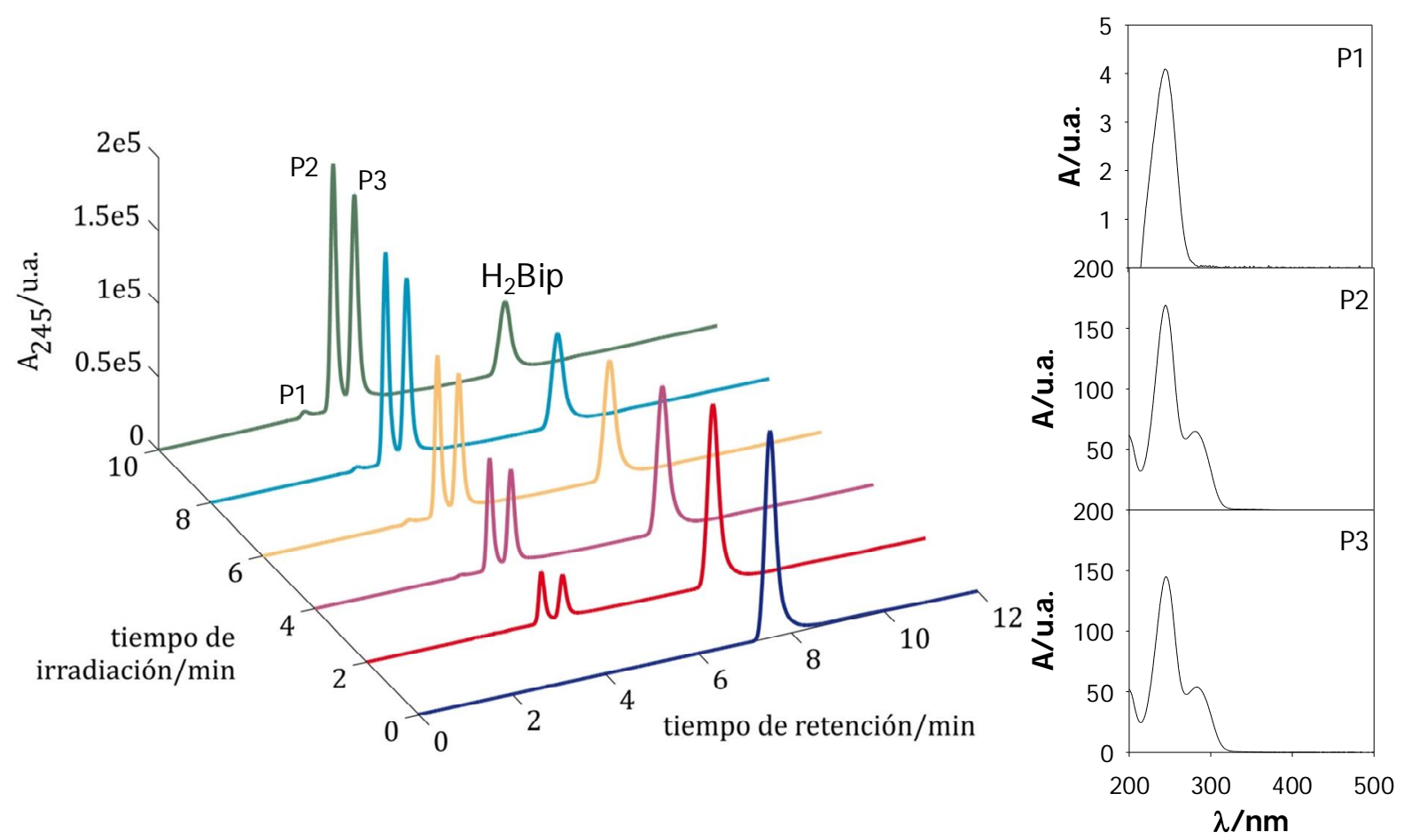

Figura 9.2 Cromatogramas $\left(\lambda_{\text {an }}=245 \mathrm{~nm}\right)$ obtenidos de la irradiación de una solución de $\mathrm{H}_{2} \mathrm{Bip}$ en ausencia de $\mathrm{O}_{2}\left(\left[\mathrm{H}_{2} \mathrm{Bip}\right]_{0}=143 \mu \mathrm{M}, \mathrm{pH}=6,7\right)$. Espectros de absorción de P1, P2 y P3. Sistema de irradiación I y Equipo HPLC I.

A partir de las áreas de los picos y de la correspondiente curva de calibración, se obtuvo el perfil de concentración de $\mathrm{H}_{2}$ Bip con respecto al tiempo de irradiación, el cual se aprecia en la Figura 9.3. Se observa claramente la disminución de la concentración del reactivo, con el consecuente aumento en el área de los productos P2 y P3. Cabe aclarar que el área del producto P1 tuvo un crecimiento despreciable en el tiempo que duraron los experimentos.

A partir de la velocidad de consumo y de la intensidad de radiación absorbida por el reactivo se calculó el rendimiento cuántico de consumo de $\mathrm{H}_{2} \mathrm{Bip}$ (Ф-H2Bip, Sección 4.4). El valor promedio calculado a partir de los resultados obtenidos en distintos experimentos fue de $\Phi_{\text {-н2Bip }}=0,053 \pm 0,003$. Este valor es muy similar al determinado para $\Phi$-H2Nep, expuesto en el capítulo anterior. 


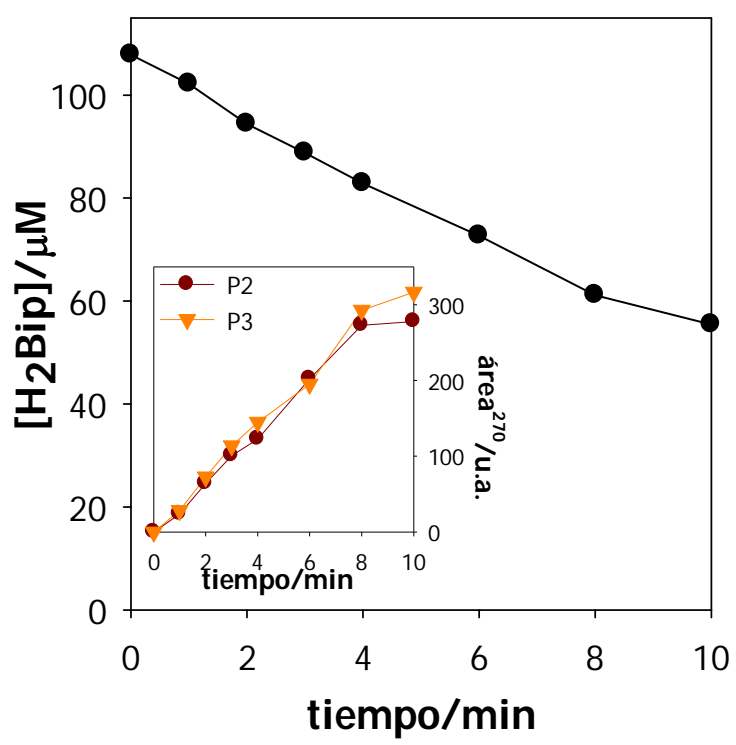

Figura 9.3 Evolución de la concentración de $\mathrm{H}_{2} \mathrm{Bip}$ con el tiempo de irradiación de una solución de $\mathrm{H}_{2} \mathrm{Bip}$ en ausencia de $\mathrm{O}_{2}\left(\left[\mathrm{H}_{2} \mathrm{Bip}\right]_{0}=108 \mu \mathrm{M} ; \mathrm{pH}=6,6\right)$. Sistema de irradiación I y Equipo HPLC I. Inset: evolución de las áreas de P2 y P3 con el tiempo de irradiación.

\subsection{Irradiación en presencia de oxígeno}

Cuando soluciones acuosas aireadas de $\mathrm{H}_{2} \operatorname{Bip}(50-200 \mu \mathrm{M}, \mathrm{pH}=7,0)$ fueron irradiadas con el Sistema de irradiación I a 350 nm (Sección 4.3), los cambios espectrales registrados fueron muy distintos a los detectados en ausencia de $\mathrm{O}_{2}$ (Figura 9.4.a), a diferencia de lo observado para $\mathrm{H}_{2} \mathrm{Nep}$. En este caso, se observó que la banda de menor energía de $\mathrm{H}_{2}$ Bip disminuyó, pero a partir de un tiempo determinado comenzó a aumentar la absorbancia a longitudes de onda mayores a $340 \mathrm{~nm}$. En esta región del espectro las pterinas oxidadas tienen su banda de absorción típica (Figura 2.5, Capítulo 2). Además, los puntos isosbésticos no permanecieron constantes durante la ventana de tiempo analizada, sugiriendo así la existencia de reacciones secundarias o la presencia de varios procesos, cuya contribución al consumo de reactivo cambia durante el experimento. En la Figura 9.4.b se observa la evolución de la absorbancia a distintas $\lambda$ mostrando la complejidad cinética de los cambios espectrales de este proceso. 

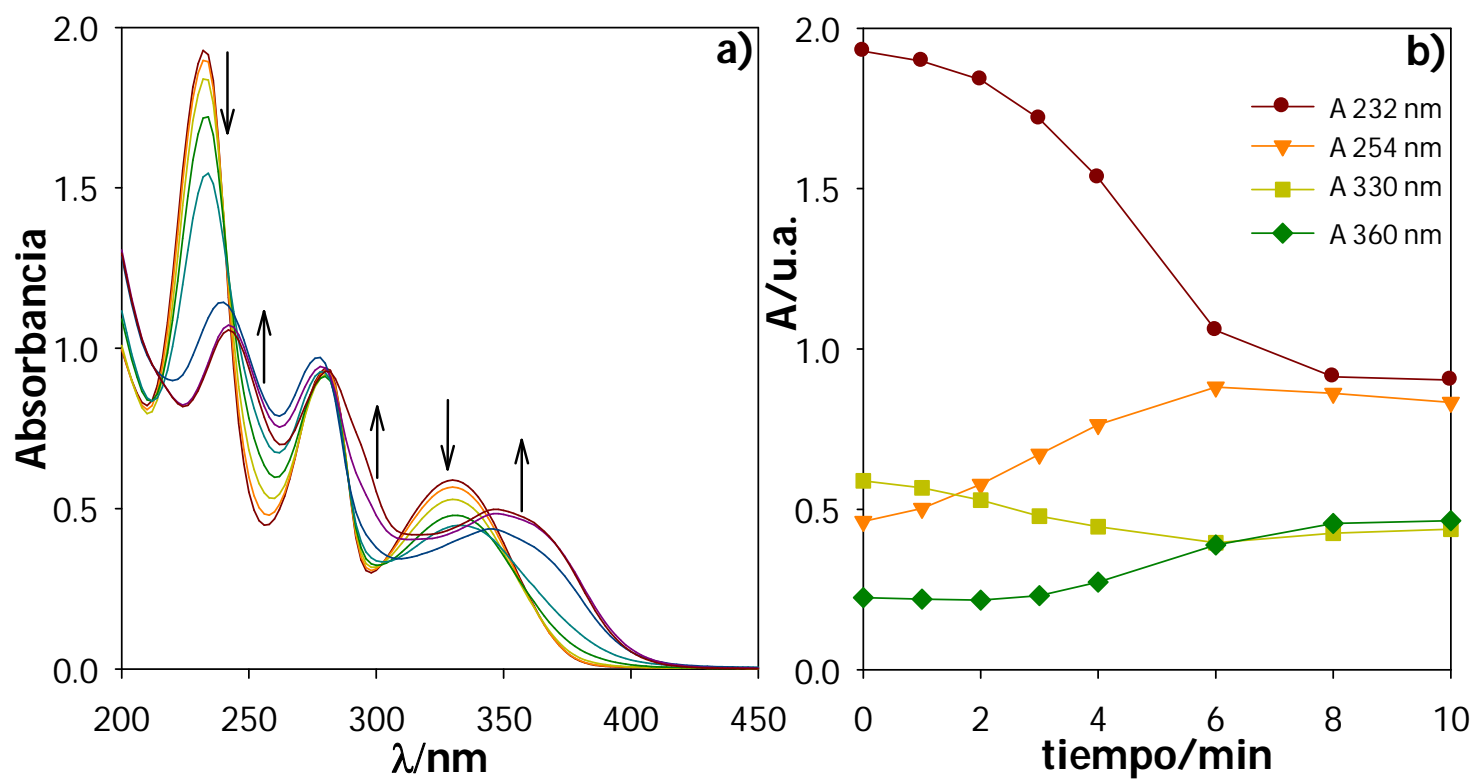

Figura 9.4 a) Evolución del espectro de absorción de una solución de $\mathrm{H}_{2} \mathrm{Bip}$ en presencia de $\mathrm{O}_{2}$ en función del tiempo de irradiación. $\left[\mathrm{H}_{2} \mathrm{Bip}\right]_{0}=100 \mu \mathrm{M}, \mathrm{pH}=7,0, \mathrm{ti}=0,1,2,3,4,5$, 6,8 y $10 \mathrm{~min}$. b) Evolución de la absorbancia a 232, 254, 330 y $360 \mathrm{~nm}$ en función del tiempo de irradiación. Camino óptico $=1 \mathrm{~cm}$. Sistema de irradiación $\mathrm{I}$.

Las muestras irradiadas se analizaron por HPLC donde se detectó la presencia de, al menos, seis fotoproductos tal como se observa en la Figura 9.5. Comparando los tiempos de retención y los espectros de absorción registrados por el detector del cromatógrafo, se deduce que tres de ellos son los mismos que se observaron en ausencia de $\mathrm{O}_{2}$ (P1, P2, P3, Figura 9.2). Con respecto a los otros tres productos, sus tiempos de retención y espectros son idénticos a los obtenidos con soluciones estándar de Bip, 6formilpterina (Fop) y 6-carboxipterina (Cap). La formación de estas pterinas oxidadas explica el aumento de absorbancia a $\lambda>340 \mathrm{~nm}$ observado en los espectros de las soluciones irradiadas (Figura 9.4), y revela la existencia de una vía de reacción oxidativa adicional a la ya descripta para la irradiación en ausencia de $\mathrm{O}_{2}$. Teniendo en cuenta las reacciones de fotooxidación descriptas en el Capítulo 6,1,2 Bip sería el primer fotoproducto en la vía oxidativa de $\mathrm{H}_{2} \mathrm{Bip} \mathrm{y}$, posteriormente, este compuesto se fotooxidaría para generar Fop, el cual, a su vez, se oxidaría fotoquímicamente a Cap. De esta manera, se pone de manifiesto una diferencia sustancial con el comportamiento fotoquímico de $\mathrm{H}_{2} \mathrm{Nep}$ ya que, como se explicó en el capítulo anterior, cuando se irradia este compuesto en presencia de $\mathrm{O}_{2}$, sólo se generan los dímeros. Cabe destacar que el espectro de Cap observado en la parte inferior de la Figura 9.5, se encuentra deformado, debido a que se superpone en tiempo de retención con P2. 

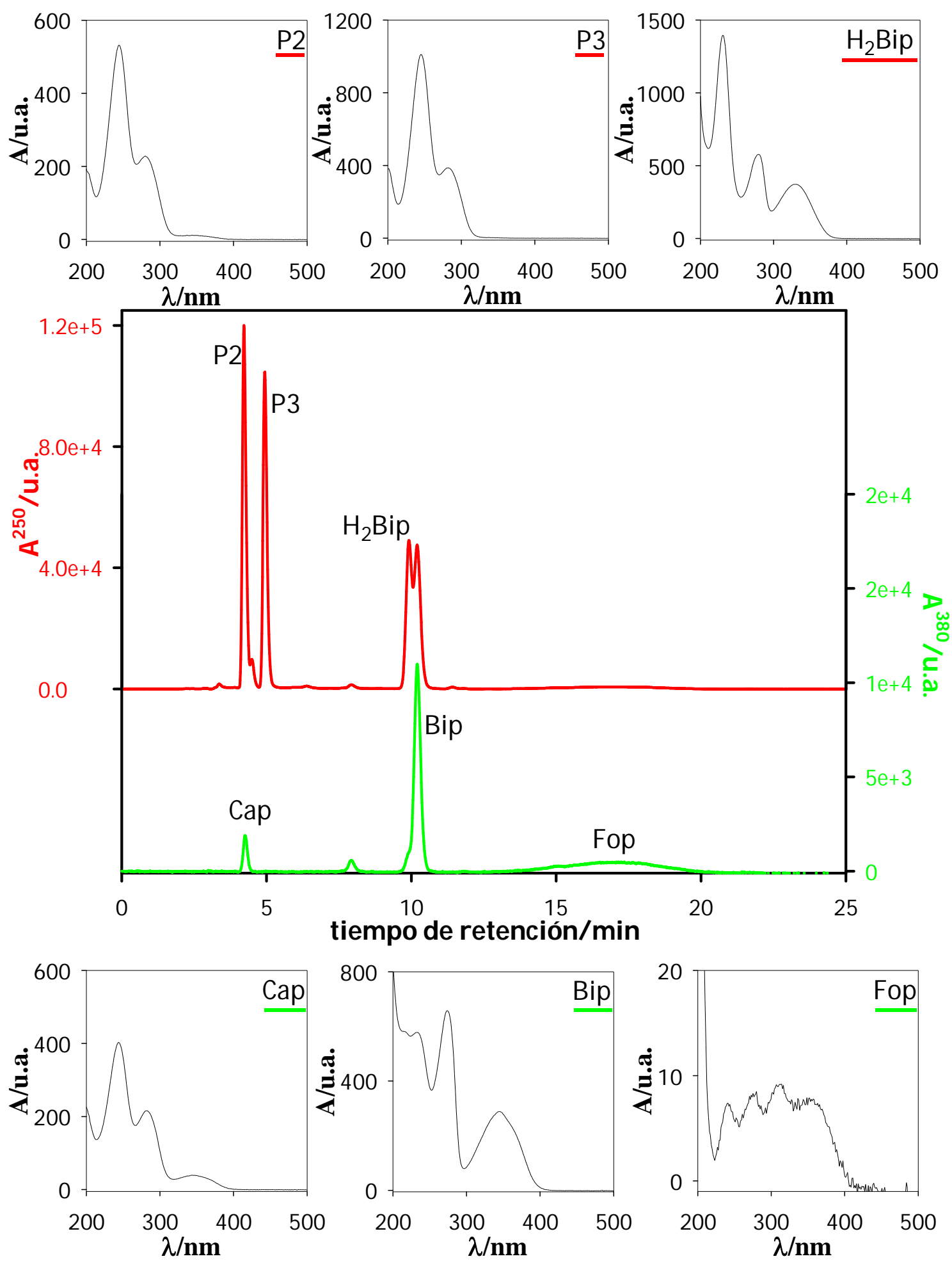

Figura 9.5 Cromatogramas de una solución de $\mathrm{H}_{2}$ Bip aireada $\left(\left[\mathrm{H}_{2} B i p\right]_{0}=144 \mu \mathrm{M}, \mathrm{pH}=7,2\right)$ irradiada por 6 min. En color rojo y color verde se muestran los cromatogramas registrados con $\lambda_{\mathrm{an}}=250 \mathrm{~nm}$ y $380 \mathrm{~nm}$, respectivamente. Espectros de absorción de cada compuesto. Sistema de irradiación Iy Equipo HPLC I.

Los perfiles de concentración muestran, como era de esperar, que tanto el consumo del reactivo como así también la producción de Bip aumentan con el tiempo de irradiación (Figura 9.6.a). Sin embargo, desde el punto de vista cinético, se observa un 
comportamiento muy particular: el consumo de $\mathrm{H}_{2}$ Bip aumenta su velocidad con el tiempo de irradiación. Vale la pena señalar que este fenómeno, el cual será analizado en detalle más adelante, no se registró durante las irradiaciones de $\mathrm{H}_{2} \mathrm{Bip}$ en ausencia de $\mathrm{O}_{2}$ (Sección 9.1). Por otro lado, se observó en distintos experimentos que la concentración de Bip alcanzaba un máximo a un determinado tiempo de irradiación, a partir del cual comenzaba a disminuir como consecuencia de su propia fotooxidación, generando Fop. Del mismo modo, la concentración de Fop alcanzaba un máximo y luego decrecía debido a su degradación fotoquímica produciendo Cap. Este comportamiento global de los perfiles de concentración, mostrado a modo de ejemplo en la Figura 9.6.a para un experimento típico, indica entonces que inicialmente $\mathrm{H}_{2}$ Bip se oxida a Bip y, a continuación, este compuesto sufre la secuencia de oxidaciones descriptas en el Capítulo 6 que conduce a la acumulación de Cap como producto final.

En el perfil de concentración de Bip mostrado en la Figura 9.6 se aprecia que la velocidad de formación de este compuesto aumenta con el tiempo de irradiación. Más aún, si se suman a cada tiempo de irradiación las concentraciones de todos los productos oxidados ([Bip]+[Fop]+[Cap]), se obtiene la concentración total de $\mathrm{H}_{2}$ Bip que reacciona a través de la vía fotooxidativa. La velocidad de formación de pterinas oxidadas aumenta con el tiempo de irradiación (Figura 9.6.b). Esto demuestra que la aceleración registrada en el consumo de reactivo se debe a un aumento en la velocidad de la vía oxidativa.

Por otro lado, el área de los picos cromatográficos de P2 y P3 muestra un continuo aumento sin aceleración (inset, Figura 9.6.a), el cual llega a un plateau que coincide lógicamente con el momento en que se consume todo el reactivo. Se puede evaluar la cinética de la vía de fotodimerización estimando para cada tiempo de irradiación la concentración de fotodímeros mediante la siguiente expresión [fotodímeros $]=\left[\mathrm{H}_{2} \mathrm{Bip}\right]_{0}-\left[\mathrm{H}_{2} \mathrm{Bip}\right]-[\mathrm{Bip}]-[\mathrm{Fop}]-[\mathrm{Cap}]$. Este balance de masa fue incluido en los perfiles de concentración de la Figura 9.6.b y puede apreciarse que, en concordancia con la evolución temporal de las áreas de P2 y P3, la concentración de estos fotodímeros calculada con la ecuación anterior, no sufre aceleración como sí sucede con la vía oxidativa. Además, queda claro que cualitativamente la fotodimerización es significativa, constituyendo alrededor del $40 \%$ del consumo total de $\mathrm{H}_{2} \mathrm{Bip}$ en el experimento de la Figura 9.6.

Por lo tanto, queda claro que existen dos vías de consumo de $\mathrm{H}_{2} \mathrm{Bip}$ en presencia de $\mathrm{O}_{2}$ : la primera es la formación de P2 y P3, la cual es de esperar ya que se observó 
previamente para $\mathrm{H}_{2}$ Bip en ausencia de $\mathrm{O}_{2}$ y para $\mathrm{H}_{2} \mathrm{Nep}$ en presencia y ausencia de $\mathrm{O}_{2}$ (Capítulo 8), mientras que la segunda, es una vía oxidativa que muestra un efecto cinético que podría calificarse como auto-fotocatalítico.

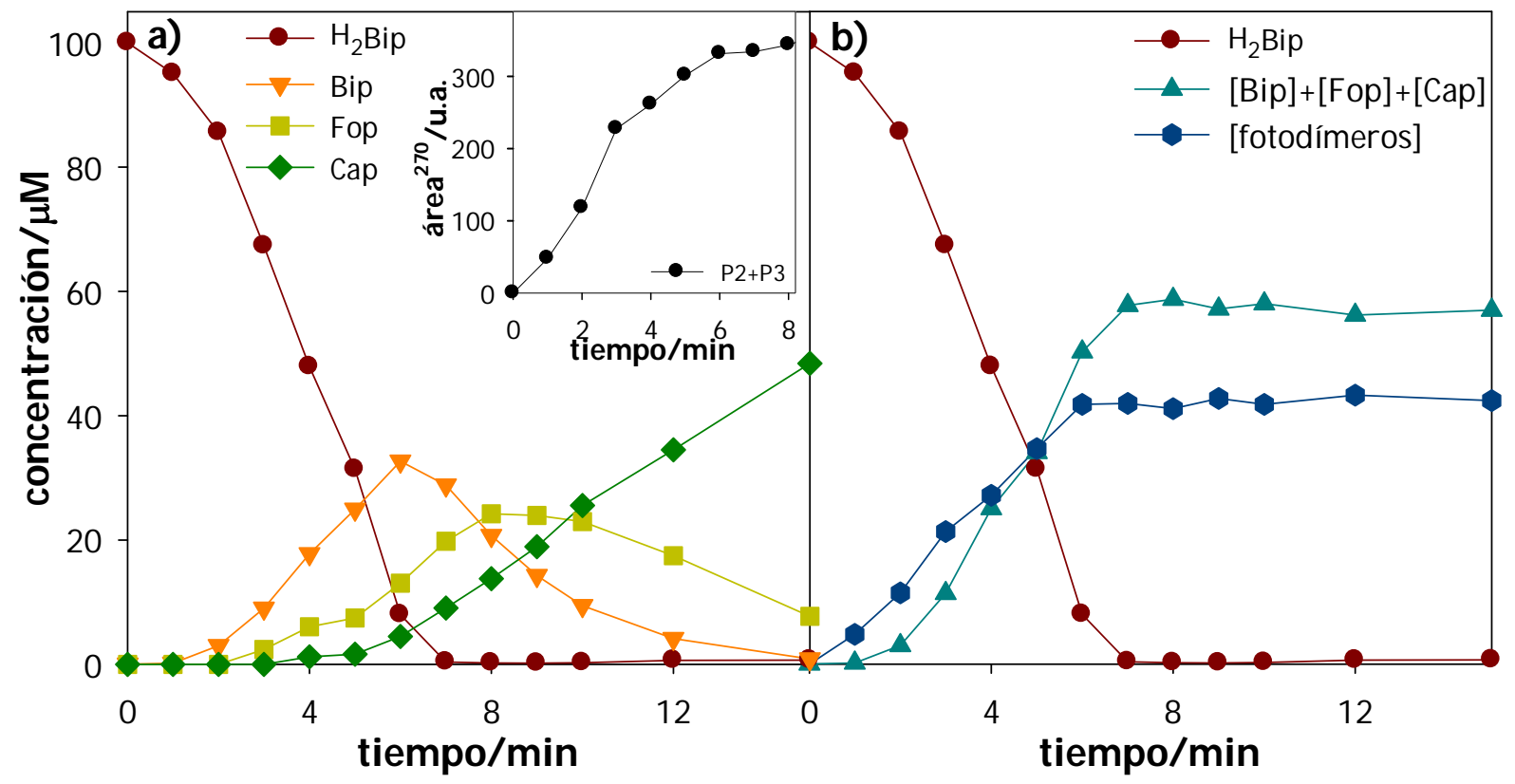

Figura 9.6 a) Evolución de las concentraciones con el tiempo de irradiación $\left(\left[\mathrm{H}_{2} \mathrm{Bip}\right]_{0}=\right.$ $100 \mu \mathrm{M}, \mathrm{pH}=7,0$ ). Inset: Evolución del área total de P2+P3 con el tiempo de irradiación. b) Evolución de la suma de Bip, Fop y Cap y de la concentración calculada de los fotodímeros a cada tiempo de irradiación. Sistema de irradiación I y Equipo HPLC I.

Debido al fenómeno de aceleración observado para la vía oxidativa, se decidió estudiar la reacción prestando mayor atención a la primera etapa del proceso. Es decir, al intervalo de tiempo durante el cual no se evidencia la fotooxidación de Bip. Para ello se repitieron experimentos realizados en condiciones similares a las del experimento presentado en la Figura 9.6, pero analizando solamente los dos primeros minutos de irradiación. Como se observa en la Figura 9.7, en esta etapa inicial la fotodimerización es la reacción principal, con una velocidad de formación de P2 y P3 constante, al igual que lo observado en condiciones de ausencia de $\mathrm{O}_{2}$. En cambio, la reacción de formación de Bip adquiere importancia a tiempos de irradiación más prolongados, experimentando un efecto auto-fotocatalítico.

Se determinó el $\Phi_{\text {-H2Bip }}($ Sección 4.4) dentro de la ventana de tiempo donde la velocidad de desaparición del reactivo es constante (1 min) (Figura 9.7), obteniéndose un valor de 0,05 $\pm 0,01$. Cabe aclarar que, al ser muy pequeño el consumo de reactivo debido a la estrecha ventana de tiempo analizada, el error en el cálculo es considerablemente elevado. Sin embargo, el valor de $\Phi$-н2Bip calculado en estas condiciones se puede considerar igual, dentro del error experimental, al determinado en 
condiciones anaeróbicas. Por otro lado, el rendimiento cuántico de producción de Bip no puede ser calculado debido a que la formación de Bip dentro del primer minuto de irradiación es prácticamente despreciable (Figura 9.7.a). Sin embargo, puede asumirse como, al menos, un orden de magnitud menor $\left(\Phi_{\text {Bip }}<0,005\right)$.
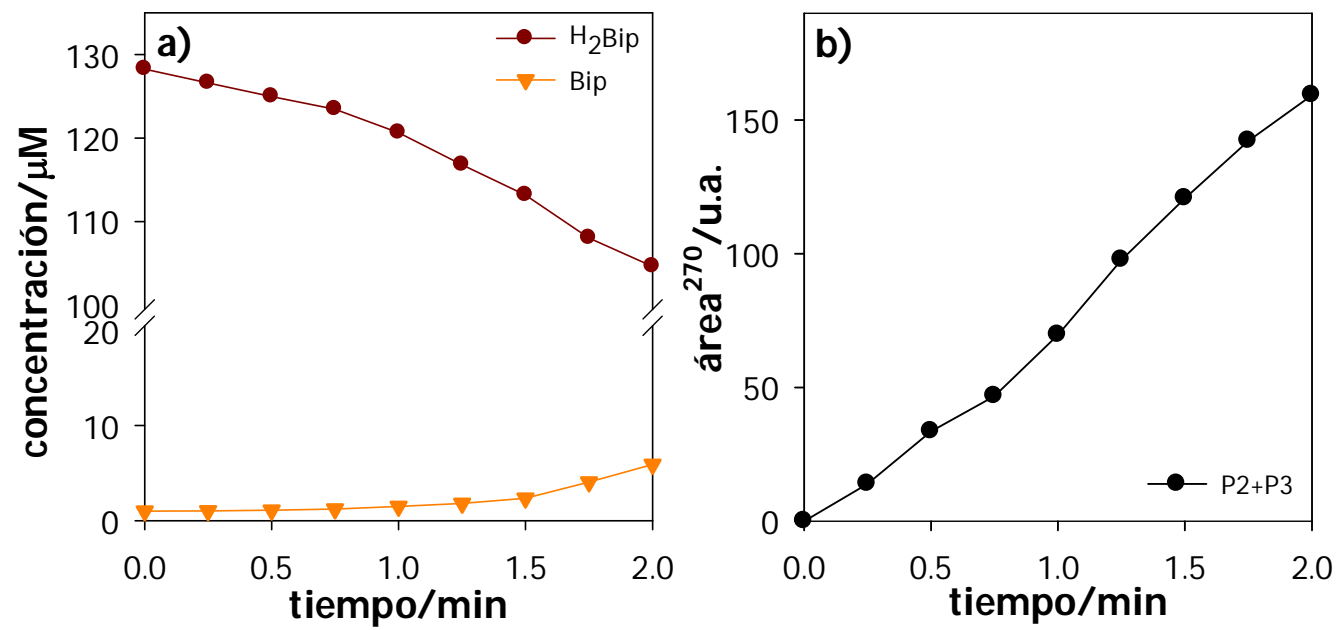

Figura 9.7 a) Evolución de las concentraciones con el tiempo de irradiación $\left(\left[\mathrm{H}_{2} \mathrm{Bip}_{0}=\right.\right.$ $128 \mu \mathrm{M}, \mathrm{pH}=6$,9). b) Evolución del área total de P2+P3 con el tiempo de irradiación. Sistema de irradiación I y Equipo HPLC I

Queda claro entonces que en la primera etapa del proceso fotoquímico, la fotodimerización predomina y ocurre con una eficiencia del orden de la calculada en condiciones anaeróbicas, lo cual coincide, a su vez, con lo descripto para $\mathrm{H}_{2} \mathrm{Nep}$ (Capítulo 8). Por otro lado, la vía oxidativa es despreciable al comienzo del proceso y adquiere importancia a tiempos de irradiación mayores, convirtiéndose en la vía predominante y siendo responsable, finalmente, de más del $50 \%$ del consumo total de $\mathrm{H}_{2}$ Bip, como fue establecido para el análisis del experimento de la Figura 9.6.

Conjuntamente con los productos cuantificados por HPLC se investigó la presencia de $\mathrm{H}_{2} \mathrm{O}_{2}$ en las soluciones irradiadas. Se detectó la formación de esta especie reactiva, con un aumento en su concentración a medida que se incrementa el tiempo de irradiación. Como ya se expuso, $\mathrm{H}_{2} \mathrm{O}_{2}$ se forma también durante la fotólisis de Bip (Capítulo 6) y Fop ${ }^{3}$ siguiendo la relación estequiométrica 1:1. Sin embargo, durante la fotólisis de $\mathrm{H}_{2} \mathrm{Bip}, \mathrm{H}_{2} \mathrm{O}_{2}$ fue detectado durante los primeros minutos de irradiación cuando la fotólisis de Bip es aún insignificante, sugiriendo que esta especie reactiva se genera simultáneamente con Bip a partir del reactivo.

Para corroborar esta hipótesis se realizó un balance de masa en varios experimentos, abarcando intervalos de tiempo de alrededor de 15 min. $\mathrm{Si}_{2} \mathrm{O}_{2}$ presente en las soluciones irradiadas de $\mathrm{H}_{2} \mathrm{Bip}$ fuese generado únicamente durante la 
fotoxidación de Bip y Fop, entonces la concentración esperada a cada tiempo debería ser igual a [Fop]+2[Cap]. Por otro lado, si $\mathrm{H}_{2} \mathrm{O}_{2}$ fuera también producido en la conversión fotoquímica de $\mathrm{H}_{2}$ Bip a Bip con relación estequiométrica 1:1, su concentración a un tiempo de irradiación determinado debería ser igual a [Bip]+2[Fop]+3[Cap]. Como se puede observar en la Figura 9.8, los resultados experimentales coinciden con la segunda hipótesis, confirmando así que $\mathrm{H}_{2} \mathrm{O}_{2}$ fue generado no solo en la fotólisis secundaria de Bip y Fop, sino también en la propia oxidación de $\mathrm{H}_{2}$ Bip a Bip.

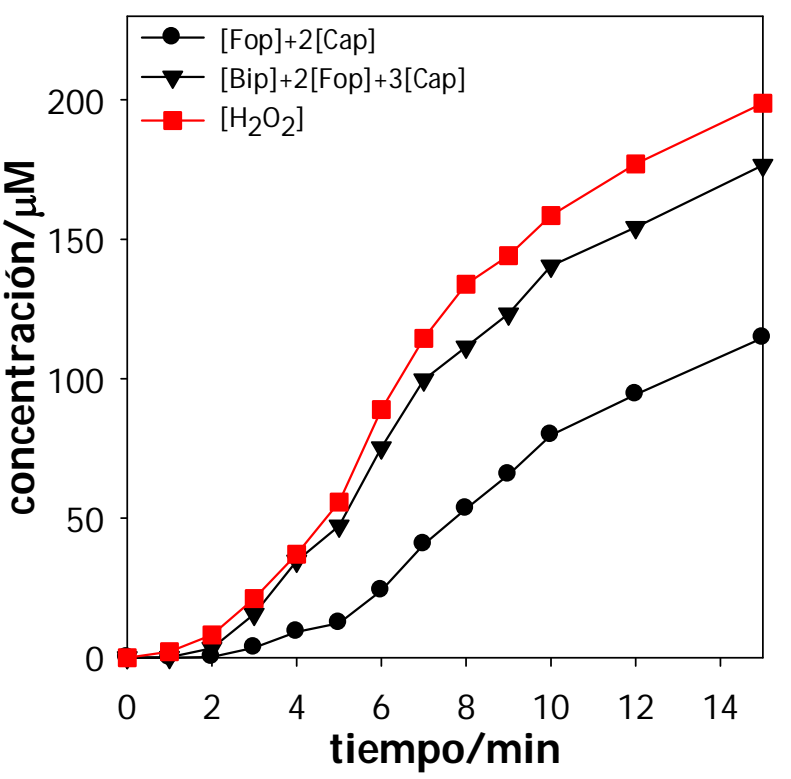

Figura 9.8 Evolución de la concentración de $\mathrm{H}_{2} \mathrm{O}_{2}$ en soluciones de $\mathrm{H}_{2} \mathrm{Bip}\left(\left[\mathrm{H}_{2} \mathrm{Bip}\right]_{0}=100\right.$ $\mu \mathrm{M}, \mathbf{p H}=\mathbf{7 , 0 )}$ con el tiempo de irradiación. Sistema de irradiación I y Equipo HPLCI.

Para completar el análisis, se realizaron medidas del consumo de $\mathrm{O}_{2}$ a medida que se irradia una solución de $\mathrm{H}_{2} \mathrm{Bip}$. Para esto se utilizó el electrodo de $\mathrm{O}_{2}$, descripto en la Sección 5.7. Como se puede observar en la Figura 9.9, la concentración de $\mathrm{O}_{2}$ va disminuyendo con el tiempo de irradiación. Asimismo, su velocidad de consumo se va incrementando, lo que coincide con el efecto auto-fotocatalítico que sufre la vía oxidativa. Análogamente a lo realizado en los capítulos anteriores, luego de finalizar la fotólisis, la solución fue analizada por HPLC, obteniéndose las concentraciones de $\mathrm{H}_{2} \mathrm{Bip}$ y pterinas oxidadas. La cantidad de $\mathrm{H}_{2} \mathrm{Bip}$ que sigue la vía oxidativan en este caso, es aproximadamente $45 \%$ del reactivo total consumido. 


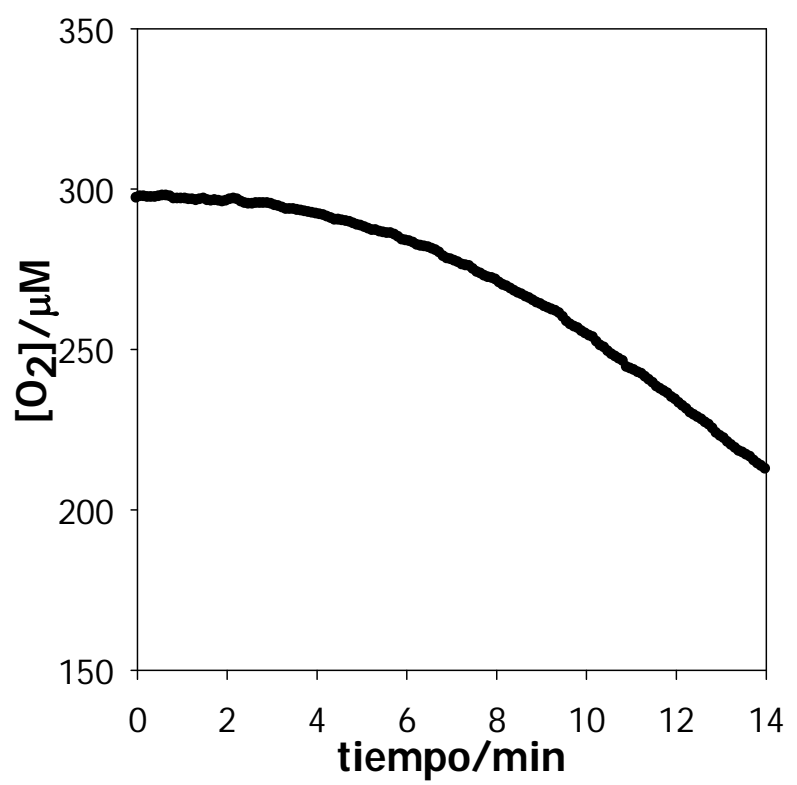

Figura 9.9 Evolución de la concentración de $\mathrm{O}_{2}$ disuelto en solución de $\mathrm{H}_{2} B i p$ con el tiempo de irradiación $\left(\left[\mathrm{H}_{2} B i p\right]_{0}=152 \mu \mathrm{M}, \mathrm{pH}=7,1\right)$. Sistema de irradiación $\mathrm{I}$.

De lo expuesto hasta aquí se desprende que la irradiación de soluciones aireadas de $\mathrm{H}_{2}$ Bip da lugar a dos vías principales de consumo de reactivo (Esquema 9.1). La primera es, muy probablemente, una fotodimerización similar a la que sufre $\mathrm{H}_{2} \mathrm{Nep}$. Esta vía es independiente del $\mathrm{O}_{2}$ presente en el medio y coincide con la observada en la reacción llevada a cabo en condiciones anaeróbicas. La segunda sólo sucede en presencia de $\mathrm{O}_{2}$ y consiste en una fotooxidación en la cual el dihidroderivado se aromatiza dando lugar a la formación de su análogo oxidado, con la consecuente generación de $\mathrm{H}_{2} \mathrm{O}_{2}$. Esta última vía de reacción sufre una aceleración después de un cierto tiempo de irradiación.

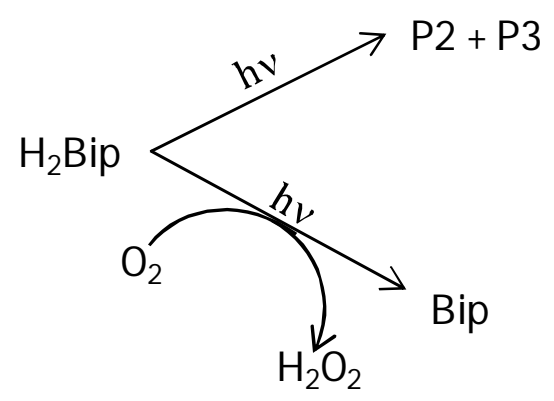

Esquema 9.1 Vías fotoquímicas de consumo de $\mathrm{H}_{2} \mathrm{Bip}$.

Para evaluar el efecto de la concentración de $\mathrm{O}_{2}$, se realizaron experimentos con soluciones saturadas en dicho gas (Sección 4.2.4). En la Figura 9.10 se presentan los perfiles de concentración del reactivo y los productos formados. Este análisis por HPLC arrojó resultados similares a los obtenidos en aire. Es decir, se observan dos vías principales de reacción: por un lado, la formación de P1, P2, P3 y, por otro, la producción 
de pterinas aromáticas (Bip, Fop y Cap). Sin embargo, se observa una menor velocidad de formación de Bip (Figura 9.10.a), acompañada por una menor producción de $\mathrm{H}_{2} \mathrm{O}_{2}$ (Figura 9.10.c). Además, analizando la reacción durante los primeros dos minutos de irradiación, se determinó un valor para el $\Phi_{\text {-H2Bip }}$ de $0,05 \pm 0,01$, igual valor dentro del error experimental a los obtenidos en aire $(\Phi$-н2вip $=0,05 \pm 0,01)$ y en ausencia de $\mathrm{O}_{2}(\Phi$. н2вір $=0,053 \pm 0,003$ ). Esto va aparejado a que la formación de dímeros, tal como en el caso de $\mathrm{H}_{2} \mathrm{Nep}$, no se encuentra inhibida por el aumento en la concentración de $\mathrm{O}_{2}$.
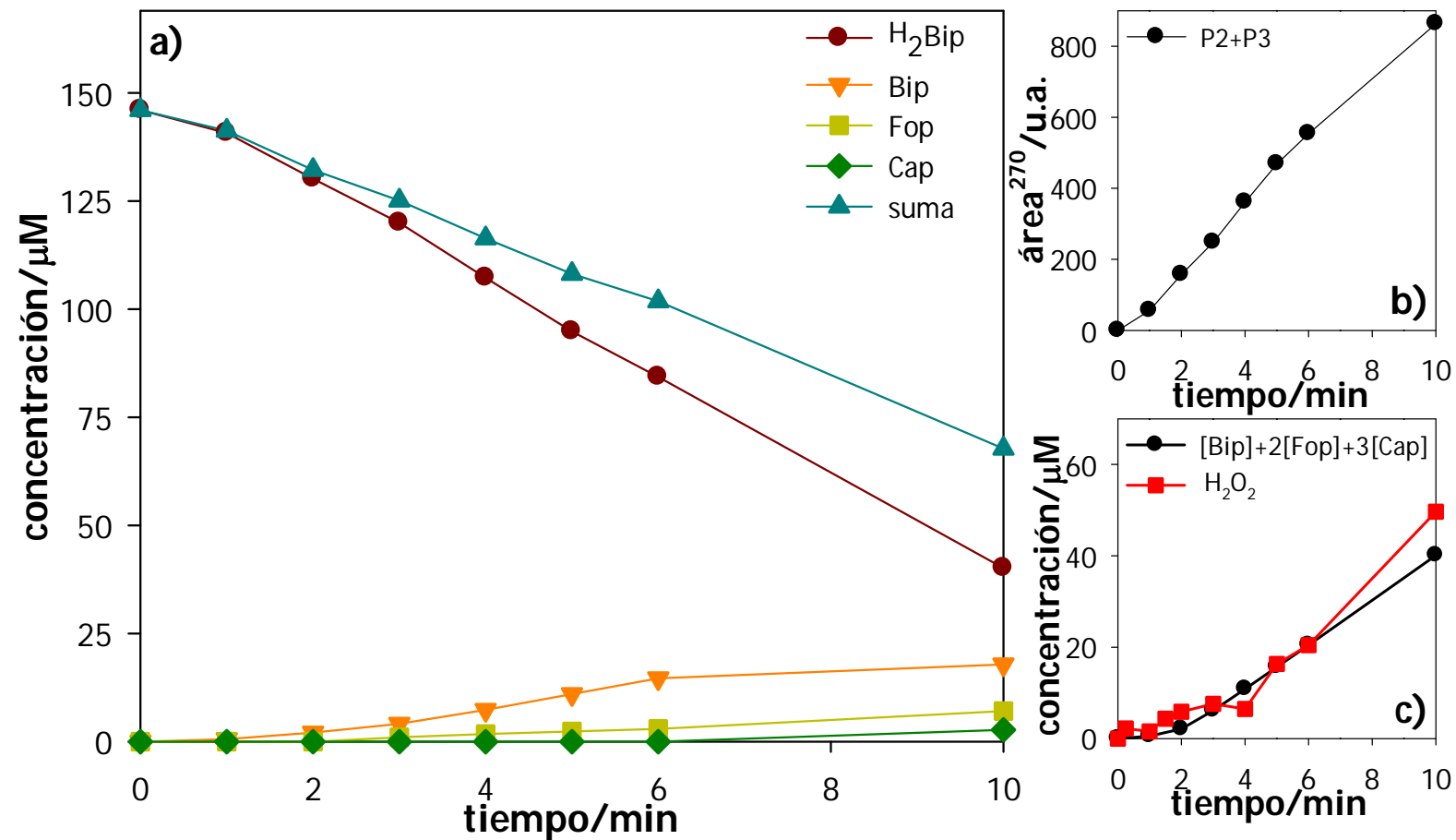

Figura 9.10 Irradiación de una solución de $\mathrm{H}_{2} \mathrm{Bip}$ saturada en $\mathrm{O}_{2}\left(\left[\mathrm{H}_{2} \mathrm{Bip}\right]_{0}=146 \mu \mathrm{M}, \mathrm{pH}=\right.$ $7,1)$. a) Evolución de las concentraciones de reactivo y productos en función del ti. Suma corresponde a la suma de concentraciones de $\mathrm{H}_{2} \mathrm{Bip}$, Bip, Fop y Cap a cada ti. b) Evolución del área total de $\mathrm{P} 2+\mathrm{P} 3$ en función del ti, c) Evolución de $\left[\mathrm{H}_{2} \mathrm{O}_{2}\right]$ y la suma de pterinas oxidadas en función del ti. Sistema de irradiación I y Equipo HPLC I.

En la Figura 9.11 se muestra en detalle los primeros tres minutos de reacción del experimento expuesto previamente. Claramente se observa como la vía oxidativa es casi despreciable comparada con la formación de los fotodímeros (Figura 9.11.b).

Sabiendo que la velocidad de consumo de $\mathrm{H}_{2}$ Bip en aire es mayor que en condiciones anaeróbicas se esperaba un incremento en la velocidad de consumo de $\mathrm{H}_{2}$ Bip en condiciones de saturación de $\mathrm{O}_{2}$. Sin embargo, en experimentos comparativos realizados en aire y en saturación de $\mathrm{O}_{2}$ (Figura 9.12) se observa que, como se mencionó anteriormente, la velocidad de formación de dímeros no se ve afectada y la vía oxidativa se encuentra inhibida. 

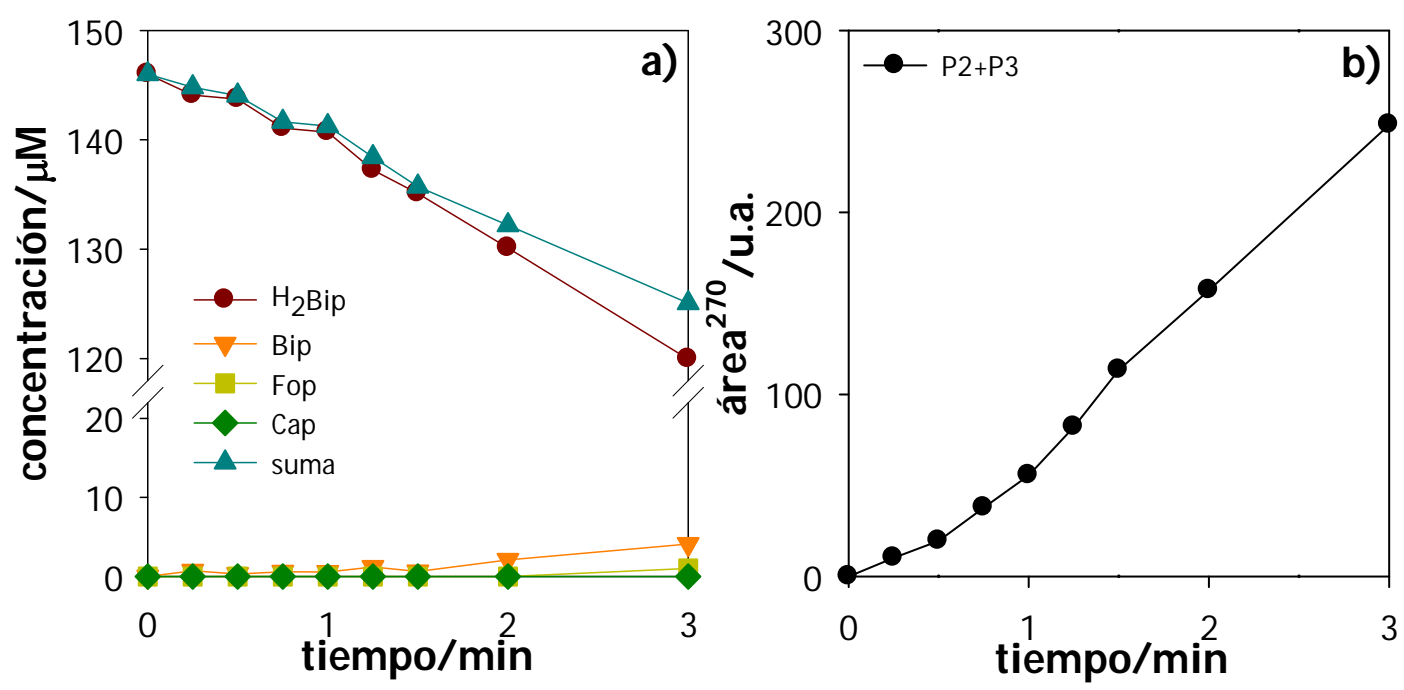

Figura 9.11 a) Evolución de las concentraciones con el tiempo de irradiación $\left(\left(\left[\mathrm{H}_{2} \mathrm{Bip}_{0}=\right.\right.\right.$ $146 \mu \mathrm{M}, \mathrm{pH}=7,1)$ Suma corresponde a la suma de concentraciones de $\mathrm{H}_{2} \mathrm{Bip}$, Bip, Fop y Cap a cada ti. b) Evolución del área total de P2+P3 con el tiempo de irradiación. Sistema de irradiación I y Equipo HPLC I

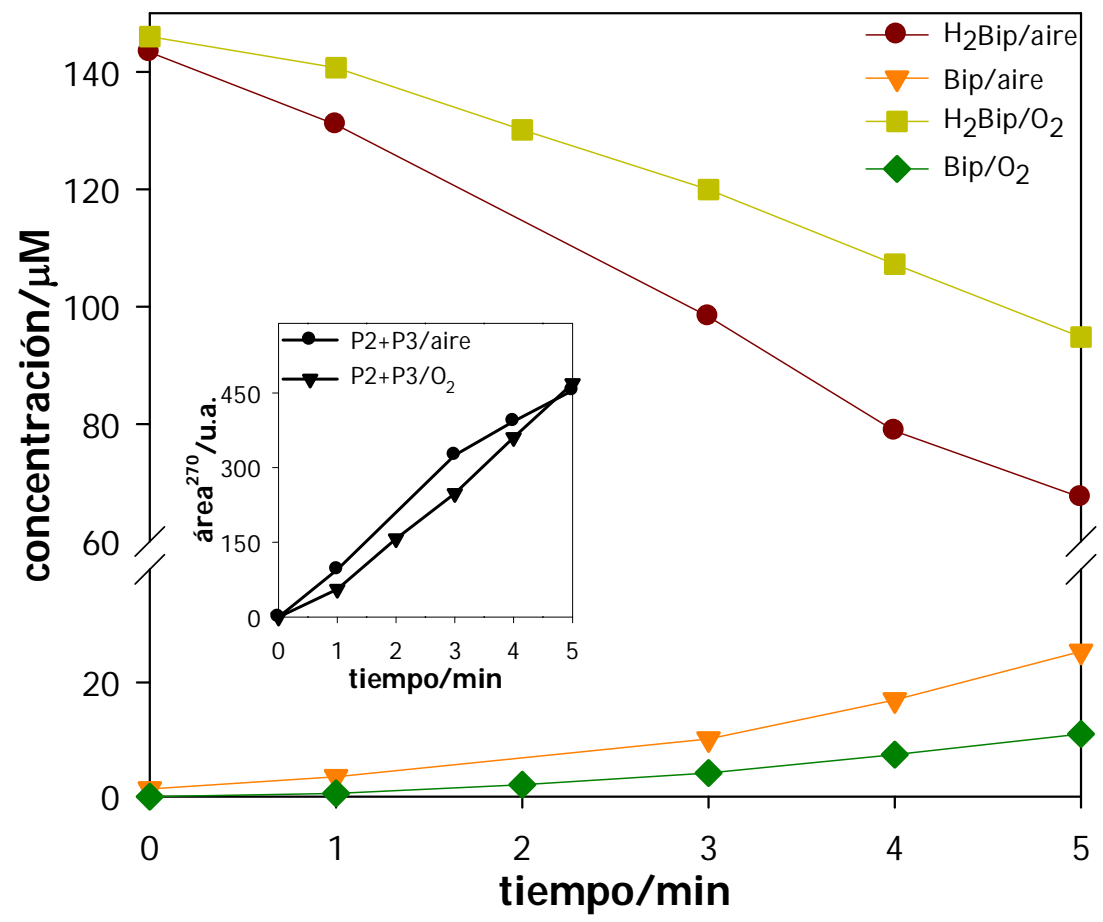

Figura 9.12 Evolución de las concentraciones de reactivos y productos durante la irradiación de soluciones de $\mathrm{H}_{2} \mathrm{Bip}$ en aire $\left(\left[\mathrm{H}_{2} \mathrm{Bip}_{0}=143 \mu \mathrm{M}, \mathrm{pH}=7,0\right)\right.$ y en atmósfera saturada en $\mathrm{O}_{2}\left(\left[\mathrm{H}_{2} \mathrm{Bip}_{0}=146 \mu \mathrm{M}, \mathrm{pH}=7,1\right)\right.$. Inset: Evolución con el tiempo de irradiación del área total de P2+P3. Sistema de irradiación I y Equipo HPLC I.

$\mathrm{Si}$ bien este comportamiento cinético podría considerarse contradictorio, el mismo ya había sido observado para otras pterinas oxidadas (Capítulo 6). Por lo tanto, se puede inferir nuevamente la participación de estados excitados tripletes para generar Bip. Es necesario tener en cuenta las especies reactivas generadas durante las distintas reacciones. Sabiendo que las dihidropterinas presentan una alta reactividad frente a 
especies oxidantes, ${ }^{4-7}$ y que Bip (Capítulo 6) y otras pterinas oxidadas producen fotoquímicamente diferentes EROs (anión superóxido $\left(\mathrm{O}_{2}{ }^{--}\right), \mathrm{H}_{2} \mathrm{O}_{2}$ y oxígeno singlete $\left.\left({ }^{1} \mathrm{O}_{2}\right)\right),{ }^{8}$ fue necesario considerar la producción fotoquímica de estas especies por Bip y la subsecuente reacción con $\mathrm{H}_{2}$ Bip como posible vía o mecanismo responsable del efecto auto-fotocatalítico observado en la vía oxidativa.

Teniendo en cuenta estudios previos, $\mathrm{H}_{2} \mathrm{O}_{2}$ puede ser descartado debido a que, por un lado, durante el tiempo que duran los experimentos el reactivo no puede ser oxidado por esta especie reactiva ya que la correspondiente constante bimolecular de reacción es relativamente baja $\left(\mathrm{k}=0,027 \pm 0,002 \mathrm{M}^{-1} \mathrm{~s}^{-1}, \mathrm{pH}=7,1 \pm 0,1 ; 37^{\circ} \mathrm{C}\right){ }^{4}$ Por otra parte, el producto de la reacción entre $\mathrm{H}_{2}$ Bip y $\mathrm{H}_{2} \mathrm{O}_{2}$ no corresponde a Bip, sino a $\mathrm{H}_{2} X a p$. Otra especie reactiva que puede reaccionar con $\mathrm{H}_{2}$ Bip es el ${ }^{1} \mathrm{O}_{2}$. Si existiera esta reacción, estaría ocurriendo una oxidación del reactivo fotosensibilizada por uno de los productos. Este punto se analizará en detalle en el capítulo siguiente.

\subsection{Análisis de los productos por espectrometría de masa}

Para investigar si los productos P2 y P3 generados durante la irradiación de $\mathrm{H}_{2}$ Bip son realmente de naturaleza dimérica, se analizaron soluciones irradiadas de este dihidroderivado por HPLC acoplado a un espectrómetro de masa con ionización por electrospray (Equipo MS I, Sección 5.4). Se decidió trabajar en condiciones de ausencia de $\mathrm{O}_{2}$, de manera tal de generar casi exclusivamente estos dos fotoproductos. Por lo tanto, soluciones de $\mathrm{H}_{2}$ Bip saturadas en argón, de concentraciones entre 120 y $150 \mu \mathrm{M}$ fueron irradiadas con el Sistema de irradiación II ( $335 \mathrm{~nm}$, Sección 4.3) durante $30 \mathrm{~min}$, y luego analizadas en modo positivo y negativo ( $\mathrm{ESI}^{+} \mathrm{y}$ ESI-, respectivamente).

Inicialmente se analizó una solución patrón de $\mathrm{H}_{2} \mathrm{Bip}$ y, como era de esperar, una señal correspondiente al ión molecular de la pterina como la especie $[\mathrm{M}-\mathrm{H}]^{-}$a $\mathrm{m} / \mathrm{z}=$ 238,2 fue registrada en modo ESI- En la Figura 9.13.a se observa el espectro tomado en modo $\mathrm{ESI}^{+}$donde se detecta el ión molecular de $\mathrm{H}_{2} \mathrm{Bip}$ como $[\mathrm{M}+\mathrm{H}]{ }^{+} \mathrm{y}$ el aducto [M+Na] ${ }^{+}$ a m/ z $=240,2$ y 262,2 , respectivamente. 

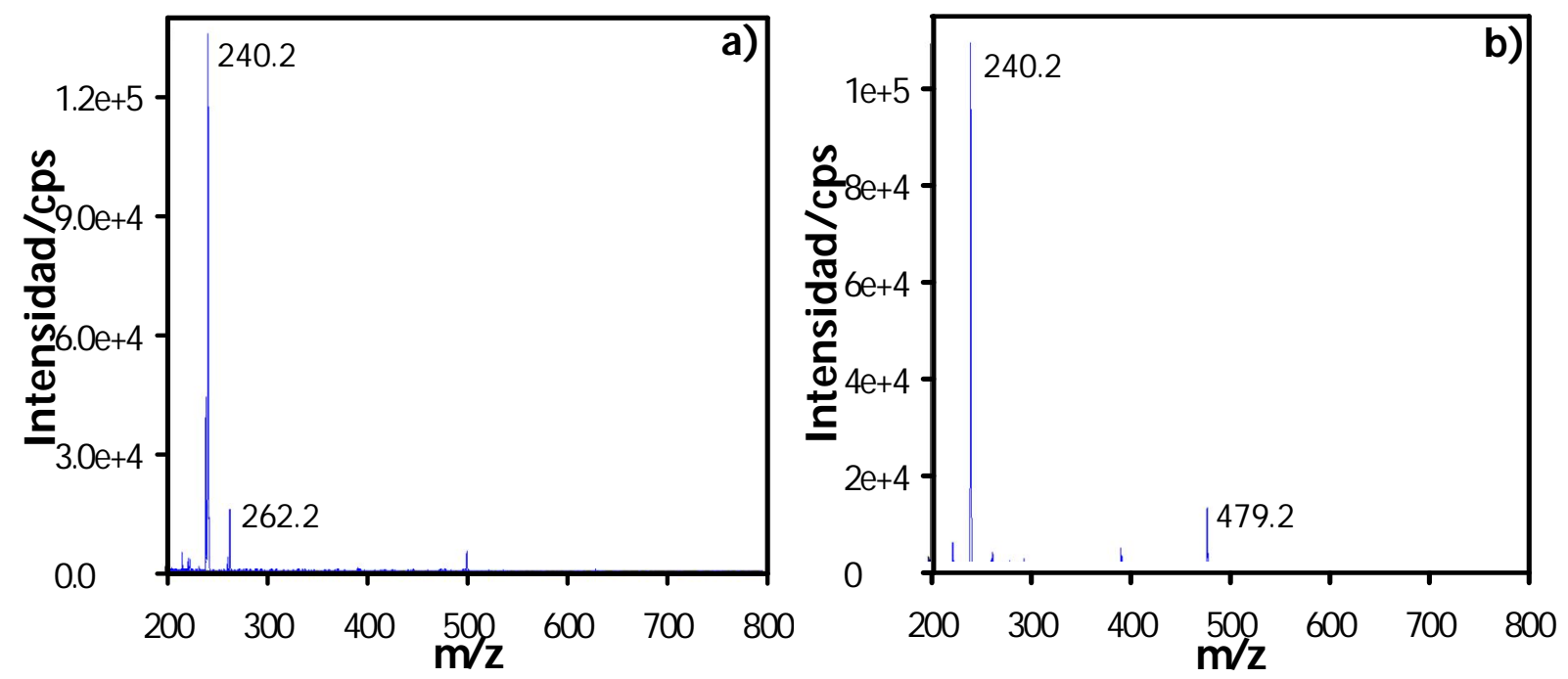

Figura 9.13 Espectros de masa (modo ESI+). a) Pico correspondiente a $\mathrm{H}_{2} \mathrm{Bip}$ en un cromatograma de la solución patrón. b) Pico correspondiente a P2 en un cromatograma de una solución de $\mathrm{H}_{2}$ Bip irradiada en condiciones anaeróbicas, ti = $30 \mathrm{~min}$. Sistema de irradiación II y Equipo MS I.

Las muestras irradiadas se analizaron en modo ESI+. En la Figura 9.13.b se muestra el espectro de masa de P2, obteniéndose un espectro casi idéntico para P3. Se obtuvo el ión molecular a $\mathrm{m} / \mathrm{z}=479,2$, que corresponde a $\left[\mathrm{M}_{2}+\mathrm{H}\right]^{+}=2 \times 239+1$. Además, se observó la presencia de un pico significativo en $\mathrm{m} / \mathrm{z}=240,2$ (igual al del reactivo). Es decir, que las masas moleculares de P2 y P3 son iguales a exactamente el doble de la masa molecular de $\mathrm{H}_{2}$ Bip, confirmando así la naturaleza dimérica también en este caso. Además, la vía más eficiente de fragmentación durante el proceso de ionización por electrospray es la rotura de estos dímeros para regenerar el monómero.

Para este dihidroderivado y sus productos también se realizaron los espectros masa/ masa (MS/ MS). Se efectuó la fragmentación del pico m/ z =240,2, para $\mathrm{H}_{2} B i p, \mathrm{P} 2$, y P3. En la Figura 9.14 se muestran solo los MS/ MS para el reactivo y P2, ya que P3 fue idéntico a P2. En la fragmentación de $\mathrm{H}_{2}$ Bip se observan señales que pueden ser asignadas a fragmentaciones específicas: un pico mayor con $\mathrm{m} / \mathrm{z}=165$ que corresponde a la pérdida del sustituyente en el $\mathrm{C} 6\left(\left[\mathrm{M}-(\mathrm{CHOH})_{2} \mathrm{CH}_{3}+\mathrm{H}\right]+\right)$, un pico a $\mathrm{m} / \mathrm{z}=222$ correspondiente a la pérdida de $\mathrm{H}_{2} \mathrm{O}\left(\left[\mathrm{M}-\mathrm{H}_{2} \mathrm{O}+\mathrm{H}\right]+\right)$ y un pico a $\mathrm{m} / \mathrm{z}=196$ correspondiente a la pérdida de $\mathrm{CO}_{2}\left(\left[\mathrm{M}-\mathrm{CO}_{2}+\mathrm{H}\right]^{+}\right)$. Es difícil especular sobre los demás fragmentos, porque sus pesos moleculares no corresponden a rupturas que se puedan interpretar fácilmente. Para los picos P2 y P3 la fragmentación registrada en las mismas condiciones, es exactamente igual a la observada para $\mathrm{H}_{2}$ Bip. Por lo tanto, se comprueba 
que cada uno de los monómeros que forman los dímeros son exactamente iguales a una molécula de reactivo.
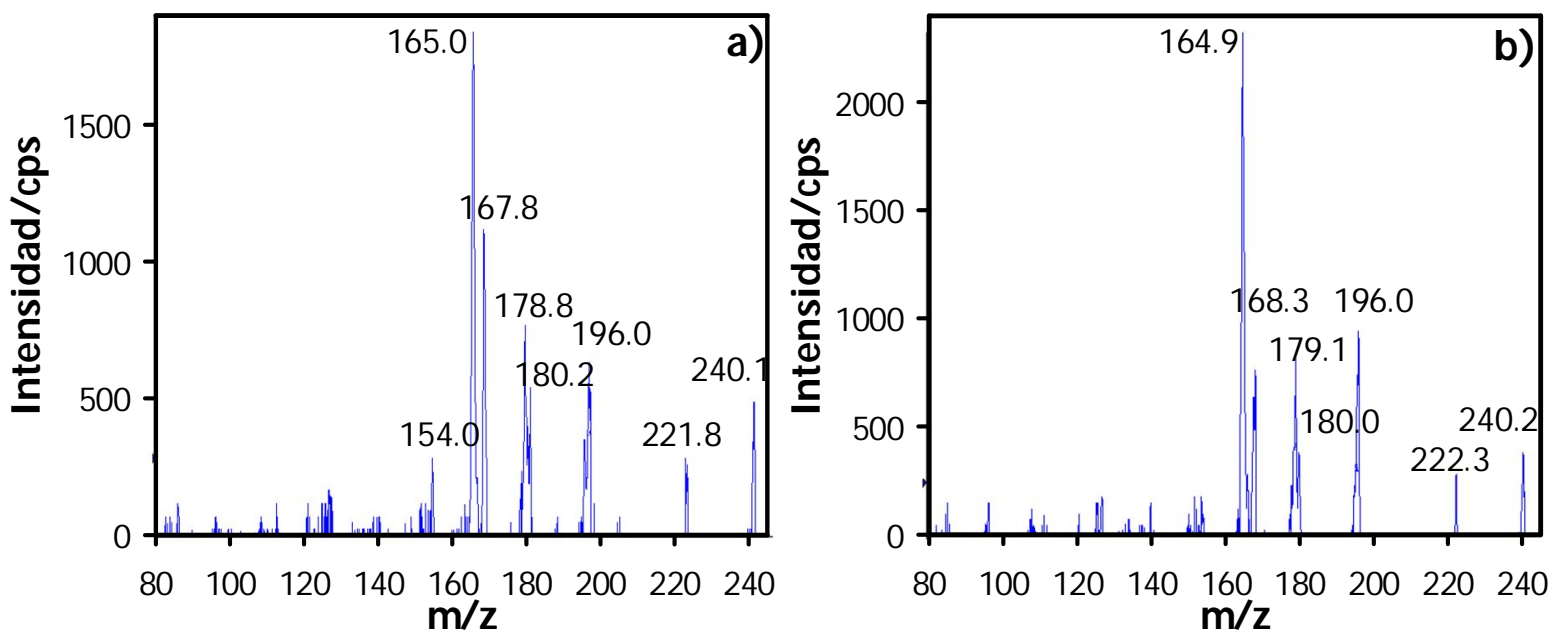

Figura 9.14 Espectros MS/ MS obtenidos en modo ESI+. a) $\mathrm{H}_{2} \mathrm{Bip}$, b) P2.

Por último, se analizaron soluciones de $\mathrm{H}_{2} \mathrm{Bip}$ irradiadas en presencia de aire. Como ya se había observado, además del reactivo y los dímeros P2 y P3, se detectaron Bip, Fop, y Cap, las cuales fueron confirmadas con los espectros de masa. La señal correspondiente al ion molecular fue detectada en los espectros de masa de los picos cromatográficos de Bip (m/z = 238,3), Fop (m/z = 192,1) y Cap (m/z = 208,1). En la Figura 9.15 se muestran estos espectros. Por lo tanto, estos resultados también reafirman la existencia de las dos vías de consumo de $\mathrm{H}_{2}$ Bip en condiciones aeróbicas: formación de dímeros y de pterinas oxidadas.

Para corroborar los pesos moleculares de los dímeros formados se continuó con el estudio tal como se describió para $\mathrm{H}_{2} \mathrm{Nep}$. Es decir, se realizaron experimentos con un espectrómetro de masa de alta resolución (Equipo MS II, Sección 5.4). Para $\mathrm{H}_{2}$ Bip se registró el ión molecular correspondiente a $[\mathrm{M}+\mathrm{H}]+$ con un valor de $\mathrm{m} / \mathrm{z}=240,1781$, el cual corresponde a una composición elemental de $\mathrm{C}_{9} \mathrm{H}_{13} \mathrm{~N}_{5} \mathrm{O}_{3}+1 \mathrm{H}$ con un error con respecto al valor teórico de 68,4 mDa. Al analizar luego la muestra de $\mathrm{H}_{2} \mathrm{Bip}$ irradiada, se procedió a calibrar el equipo con el valor teórico de la masa del reactivo previamente calculada $\mathrm{y}$, posteriormente, se determinaron los valores $\mathrm{m} / \mathrm{z}$ para los picos correspondientes a los dímeros. Para los dos productos P2 y P3 se observó el mismo ión molecular con valor de $\mathrm{m} / \mathrm{z}=479,2098$, que corresponde a $\left[\mathrm{M}_{2}+\mathrm{H}\right]+$ con composición elemental de $\mathrm{C}_{18} \mathrm{H}_{26} \mathrm{~N}_{10} \mathrm{O}_{6}+1 \mathrm{H}$ y un error con el valor teórico de -1,7 mDa (Figura 9.16). $\mathrm{Al}$ igual que para $\mathrm{H}_{2} \mathrm{Nep}$, estos resultados ratifican la producción de dímeros a partir de la fotólisis de $\mathrm{H}_{2}$ Bip. La estructura de los productos será analizada en el Capítulo 11. 

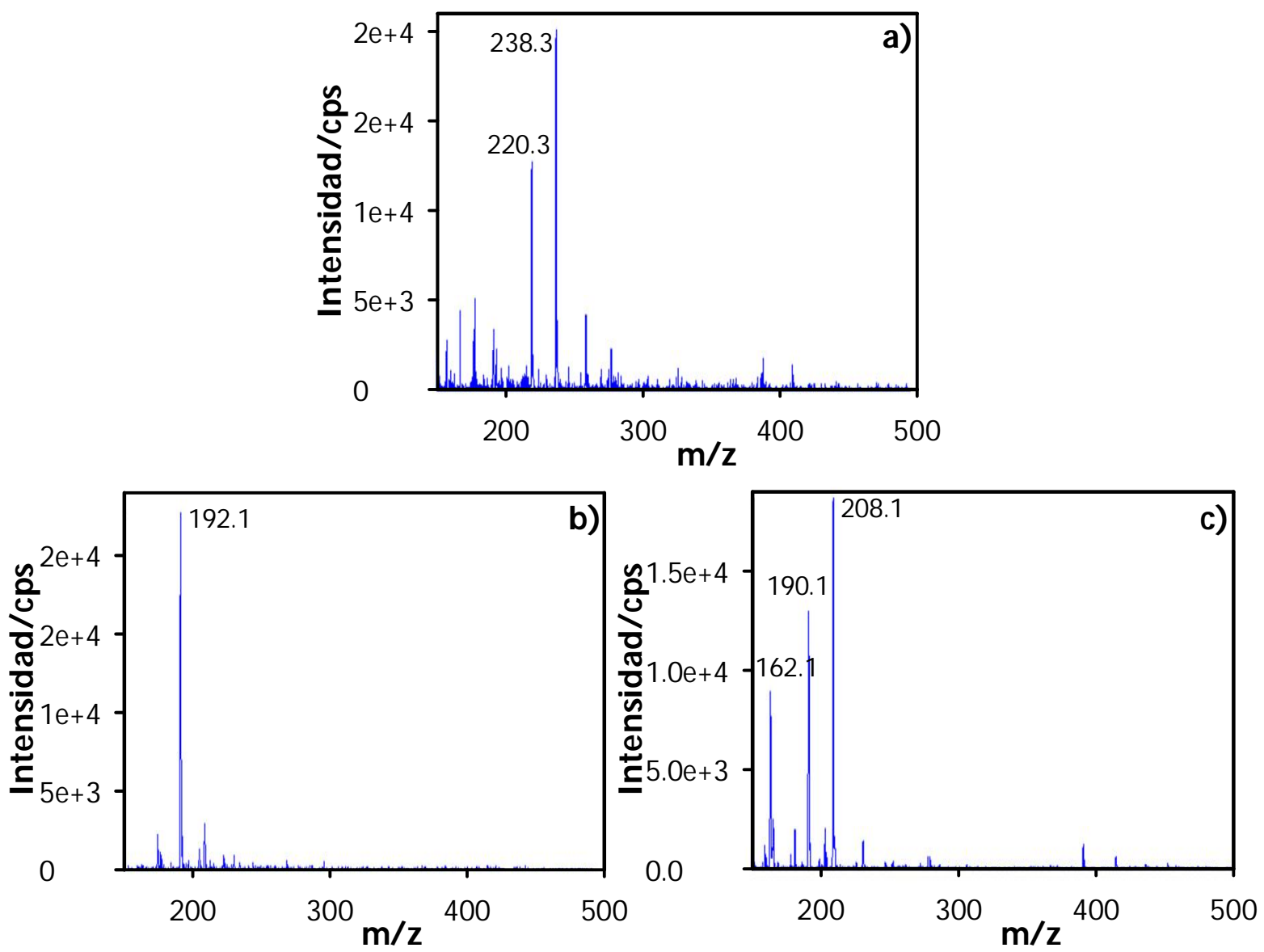

Figura 9.15 Espectros de masa (modo $\left.\mathrm{ESI}^{+}\right)$de los productos oxidados de una solución de $\mathrm{H}_{2} \mathrm{Bip}$ irradiada en presencia de $\mathrm{O}_{2}(\mathrm{ti}=30 \mathrm{~min})$. a) Pico correspondiente a Bip, b) Pico correspondiente a Fop, c) Pico correspondiente a Cap. Sistema de irradiación II y Equipo MS I.

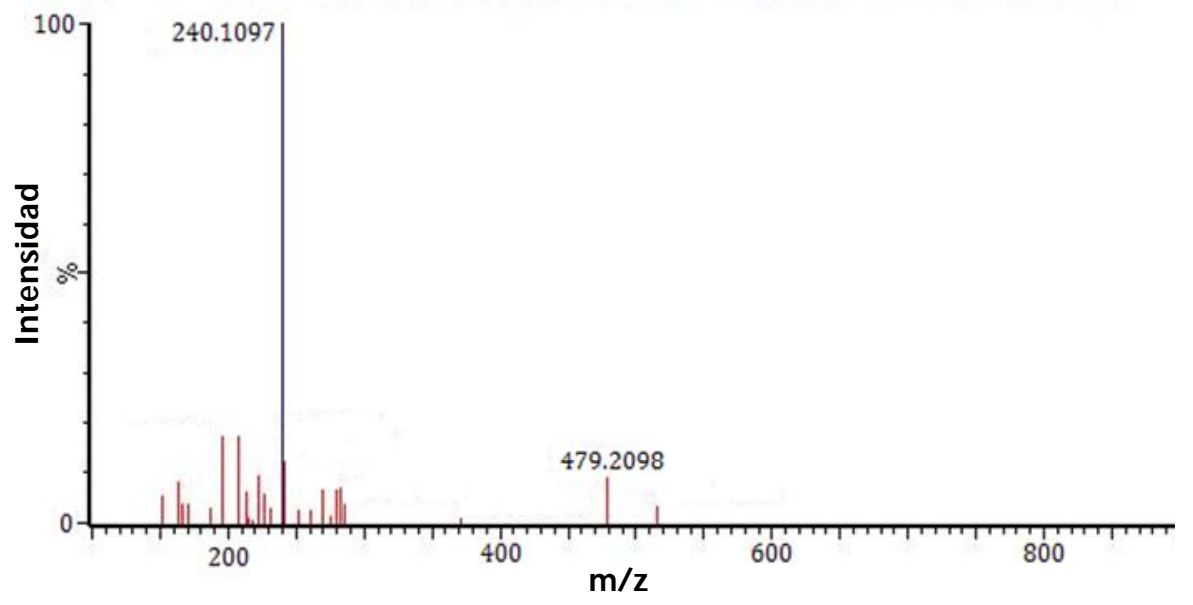

Figura 9.16 Espectros de masa (modo ESI+) de P2 de una solución de $\mathrm{H}_{2} B i p$ irradiada en ausencia de $\mathrm{O}_{2}$ (ti = 30 min). Sistema de irradiación II y Equipo MS II. 


\subsection{Implicancias biológicas de las propiedades fotoquímicas de $\mathrm{H}_{2} \mathrm{Bip}$}

Como ya se mencionó en el Capítulo 2, el vitiligo es un trastorno común de la piel caracterizado por la pérdida de la pigmentación. Se manifiesta como manchas blancas causadas por la disfunción de los melanocitos y la consecuente disminución en la biosíntesis de melanina, el pigmento natural de la piel. ${ }^{9}$ En el tejido afectado existe un desequilibrio en las concentraciones normales de los distintos componentes de esta vía. La concentración de $\mathrm{H}_{4} \mathrm{Bip}$ se eleva, debido al aumento en la síntesis de novo y a la alteración de la vía de reciclado. ${ }^{10}$ Esto conlleva a la acumulación de $\mathrm{H}_{2}$ Bip y a un aumento en la concentración de $\mathrm{H}_{2} \mathrm{O}_{2}$. Por lo tanto, es de gran importancia conocer las reacciones químicas que puede sufrir $\mathrm{H}_{2} \mathrm{Bip}$.

Tal como lo han demostrado numerosos estudios, las especies tóxicas ${ }^{11}$ y fototóxicas ${ }^{8,12}$ son las pterinas oxidadas. De éstas, Bip y Cap fueron aisladas de la piel de pacientes que sufren vitíligo, ${ }^{13}$ por lo que puede especularse que tras la formación de Bip a partir de $\mathrm{H}_{2}$ Bip y debido a la falta de protección contra la radiación UV, la misma sufre la vía fotooxidativa presentada en el Capítulo 6. Un punto que no ha sido resuelto hasta el momento es cómo se genera Bip en la piel.

Por un lado, es sabido que la oxidación de $\mathrm{H}_{2}$ Bip a partir del $\mathrm{O}_{2}$ disuelto en el medio, lleva a la formación de $\mathrm{H}_{2}$ Xap como producto principal (>80 \%) y $\mathrm{H}_{2} \mathrm{Fop}$ como producto minoritario. ${ }^{14}$ Se propuso que Bip podría formarse en la piel por reacción de $\mathrm{H}_{2}$ Bip con $\mathrm{H}_{2} \mathrm{O}_{2},{ }^{15}$ pero trabajos recientes demostraron que a partir de esta reacción se genera aproximadamente $90 \%$ de $\mathrm{H}_{2}$ Xap. ${ }^{4}$ En consecuencia, y al existir, como ya se mencionó, una falta de protección en la piel contra la radiación UV debido a la ausencia de melanina, lo más probable es que la vía fotoquímica, descripta en este capítulo, sea la causante de la generación de Bip a partir de $\mathrm{H}_{2} \mathrm{Bip}$. Como además de Bip, se genera $\mathrm{H}_{2} \mathrm{O}_{2}$ esta vía también contribuye al aumento de la concentración de esta especie reactiva. De esta manera, se puede concluir que el estudio fotoquímico de $\mathrm{H}_{2} \mathrm{Bip}$ tiene gran relevancia para la investigación bioquímica de esta enfermedad, ya que hasta el momento es la única vía posible de generación de pterinas oxidadas.

\subsection{Conclusiones}

En este capítulo se ha demostrado que cuando $\mathrm{H}_{2}$ Bip absorbe radiación UV-A, dos vías de consumo son observadas. Por un lado, sin la intervención del $\mathrm{O}_{2}$, a partir del 
estado excitado singlete y mediante la generación de un intermediario, $\mathrm{H}_{2} \mathrm{Bip}$ se fotodimeriza generando al menos dos dímeros. Por otra parte, y solo en presencia de $\mathrm{O}_{2}$, $\mathrm{H}_{2}$ Bip se oxida produciendo su análogo oxidado (Bip). En las condiciones experimentales empleadas, esta vía oxidativa contribuye muy poco al consumo de $\mathrm{H}_{2} \mathrm{Bip}$ en una primera etapa, pero adquiere relevancia al transcurrir el proceso fotoquímico. Es decir, existe un fenómeno que podría considerarse como auto-fotocatalítico que produce una aceleración en la fotooxidación del reactivo. En esta vía oxidativa participarían estados excitados tripletes y, además, se genera $\mathrm{H}_{2} \mathrm{O}_{2}$. El mecanismo propuesto para estas reacciones se muestra en el Esquema 9.2. Debido a que la fotodimerización es la vía predominante al inicio del proceso, los rendimientos cuánticos de consumo de reactivo, arrojan el mismo valor, dentro del error experimental, para las distintas atmósferas de $\mathrm{O}_{2}\left(0,053 \pm 0,003 ; 0,05 \pm 0,01\right.$ y 0,05 $\pm 0,01$, para ausencia de $\mathrm{O}_{2}$, solución equilibrada en aire y saturada en $\mathrm{O}_{2}$, respectivamente) y fueron del orden de los calculados para $\mathrm{H}_{2} \mathrm{Nep}$.

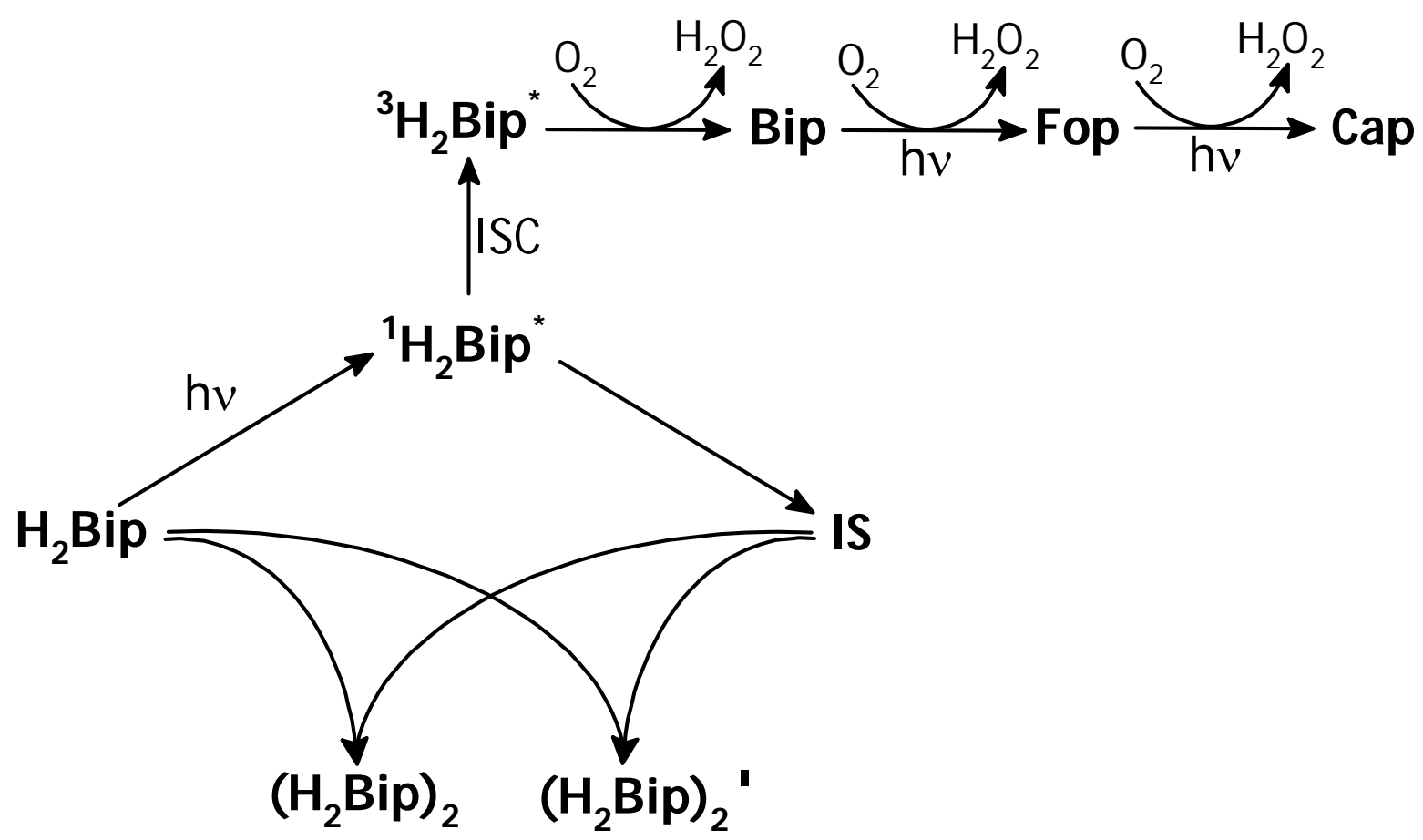

\section{Esquema 9.2 Mecanismo de degradación sufrido por $\mathrm{H}_{2}$ Bip bajo irradiación UV-A}

\subsection{Referencias}

1. A. H. Thomas, G. Suárez, F. M. Cabrerizo, R. Martino and A. L. Capparelli, Study of the photolysis of folic acid and 6-formylpterin in acid aqueous solutions, Journal of Photochemistry and Photobiology A: Chemistry, 2000, 135, 147-154.

2. R. Mengel, W. Pfleiderer and W. R. Knappe, Norrish type II analogous photodegradation of neopterin and biopterin to 5,8 -dihydropterin-6-aldehyde, Tetrahedron Lett., 1977, 18, 2817-2820. 
3. G. Petroselli, J. M. Bartsch and A. H. Thomas, Photoinduced generation of $\mathrm{H}_{2} \mathrm{O}_{2}$ and $\mathrm{O}_{2} *$ by 6formylpterin in aqueous solutions, Pteridines, 2006, 17, 82-89.

4. M. L. Dántola, T. M. Schuler, M. P. Denofrio, M. Vignoni, A. L. Capparelli, C. Lorente and A. H. Thomas, Reaction between 7,8-dihydropterins and hydrogen peroxide under physiological conditions, Tetrahedron, 2008, 64, 8692-8699.

5. M. L. Dántola, A. H. Thomas, A. M. Braun, E. Oliveros and C. Lorente, Singlet oxygen $\left(\mathrm{O}_{2}\left({ }^{1} \Delta_{\mathrm{g}}\right)\right)$ quenching by dihydropterins, Journal of Physical Chemistry A, 2007, 111, 4280-4288.

6. R. S. Shen, Inhibition of Luminol-Enhanced Chemiluminescence by Reduced Pterins, Archives of Biochemistry and Biophysics 1994, 310, 60-63.

7. G. Weiss, D. Fuchs, A. Hausen, G. Reibnegger, E. R. Werner, G. Werner-Felmayer, E. Semenitz, M. P. Dierich and $\mathrm{H}$. Wachter, Neopterin modulates toxicity mediated by reactive oxygen and chloride species, FEBS Letters, 1993, 321, 89-92.

8. A. H. Thomas, C. Lorente, A. L. Capparelli, C. G. Martínez, A. M. Braun and E. Oliveros, Singlet oxygen $\left({ }^{1} \Delta_{\mathrm{g}}\right)$ production by pterin derivatives in aqueous solutions, Photochemical and Photobiological Sciences, 2003, 2, 245-250.

9. S. J. Glassman, Vitiligo, reactive oxygen species and T-cells, Clinical Science, 2010, 120, 99-120.

10. J. E. Ayling, I. Rebrin, B. Thöny and S. W. Bailey, Mechanism of Dehydration by the Bifunctional Protein, 4a-Hydroxy-tetrahydropterin Dehydratase/ DCoH, in Chemistry and Biology of Pteridines and Folates, ed. W. Pfleiderer and R. Rokos, Blackwell Science, Berlin, 1997, pp. 565-570.

11. K. U. Schallreuter, G. Büttner, M. R. Pittelkow, J. M. Wood, N. N. Swanson and C. Korner, Cytotoxicity of 6-biopterin to human melanocytes, Biochem. Biophys. Res. Commun., 1994, 204, 43-48.

12. M. P. Denofrio, C. Lorente, T. Breitenbach, S. Hatz, F. M. Cabrerizo, A. H. Thomas and P. R. Ogilby, Photodynamic Effects of Pterin on HeLa Cells, Photochemistry and Photobiology, 2011.

13. H. Rokos and K. Schallreuter, Photo-oxidation of sepiapterin produces pterin-6-carbolxylic acid and $\mathrm{H}_{2} \mathrm{O}_{2}$ in vitro, in Chemistry and biology of pteridines and folates, ed. S. Milstien, G. Kapatos, R. A. Levine and B. Shane, Kluwe Academic, 2002, pp. 55-59.

14. M. L. Dántola, M. Vignoni, A. L. Capparelli, C. Lorente and A. H. Thomas, Stability of 7,8-dihydropterins in air-equilibrated aqueous solutions, Helvetica Chimica Acta, 2008, 91, 411-425.

15. H. Rokos, W. D. Beazley and K. U. Schallreuter, Oxidative stress in vitiligo: Photo-oxidation of pterins produces $\mathrm{H}_{2} \mathrm{O}_{2}$ and pterin-6-carboxylic acid, Biochem. Biophys. Res. Commun., 2002, 292, 805-811. 


\section{Capítulo 10}

\section{Oxidación fotosensibilizada de dihidropterinas}

En el capítulo anterior se expuso que 7,8-dihidrobiopterina $\left(\mathrm{H}_{2} \mathrm{Bip}\right)$ en presencia de $\mathrm{O}_{2}$ sufre fotooxidación para generar biopterina (Bip) y $\mathrm{H}_{2} \mathrm{O}_{2}$. Este proceso, en las condiciones experimentales utilizadas, aumenta su velocidad con el tiempo de irradiación. Este efecto auto-fotocatalítico podría tener su origen, tal como ya se sugirió en el Capítulo 9, en un proceso de fotosensibilización en el que los productos estarían involucrados. Es decir, algún producto absorbería parte de la radiación incidente y el mismo, desde su estado electrónico excitado sería capaz de reaccionar con el reactivo, induciendo su oxidación. Este proceso tendría como consecuencia un aumento global de la velocidad de consumo de $\mathrm{H}_{2}$ Bip a medida que el producto fotosensibilizador se va acumulando en el medio de reacción. Tal como se explicó en el Capítulo 1, existen diferentes mecanismos a través de los cuales un compuesto puede actuar como fotosensibilizador frente a otro.

En este capítulo se propone investigar si el efecto auto-fotocatalítico de la vía oxidativa de $\mathrm{H}_{2}$ Bip es, en verdad, consecuencia de un proceso fotosensibilizado por productos y dilucidar los mecanismos de reacción que operan en el mismo. Finalmente, en el contexto de las reacciones fotosensibilizadas, se volverá a analizar el caso de $\mathrm{H}_{2} \mathrm{Nep}$ y se intentará aportar información que explique, al menos parcialmente, su comportamiento tan diferente al de $\mathrm{H}_{2} \mathrm{Bip}$.

\subsection{Análisis de la participación de los estados excitados}

El anión ioduro (I-) es capaz de desactivar selectivamente los estados excitados singlete o triplete de compuestos orgánicos, mediante procesos de transferencia de electrones. De esta manera, se puede utilizar I- para determinar la multiplicidad de espin de los distintos estados excitados de un compuesto que participa en una reacción fotoquímica. El efecto resultante del I- en el comportamiento fotofísico de un dado compuesto depende de muchos factores y es difícil establecer generalizaciones ${ }^{1}$ Particularmente para los estados excitados tripletes, en algunos casos, la presencia de Icausa un aumento en el rendimiento cuántico de formación de triplete, mientras que, por el contrario, en otros casos se observa una disminución. Además, para ciertos 
compuestos, el anión I- presenta una cierta especificidad o eficacia de desactivación diferencial entre los estados tripletes y singletes. Tal es el caso de flavinas, donde la eficacia del I- en la desactivación de sus estados tripletes es mucho más alta que la desactivación de los respectivos estados singletes. Esta propiedad fue utilizada para investigar el rol de los estados excitados de las flavinas en los mecanismos de reacción de procesos fotoquímicos. ${ }^{2,3}$ Asumiendo que las pterinas debían comportarse en forma similar a las flavinas, Kritsky et al. realizaron experimentos de fotólisis en presencia de Ipara evaluar la participación del estado triplete de las pterinas en su fotoreducción.4 Además, en estudios de fosforescencia de pterinas adsorbidas en papel, a temperatura ambiente, se observó que el decaimiento no radiativo desde el estado triplete es aumentado por el I- 5

Para aplicar los conceptos descriptos en el párrafo anterior al estudio de la participación de los estados tripletes en la fotoquímica de $\mathrm{H}_{2} \mathrm{Bip}$, se deben realizar fotólisis en presencia de I- en concentraciones tales que dicho anión desactive a los estados tripletes, pero no a los singletes. Por lo tanto, es necesario establecer la concentración crítica de I- a partir de la cual comienza a desactivar en forma significativa a los estados excitados singletes de $\mathrm{H}_{2} \mathrm{Bip}$ y Bip. Para esto, se realizaron experimentos de desactivación de fluorescencia utilizando el equipo descripto en la Sección 5.2. Se registraron espectros de fluorescencia de soluciones de ambas pterinas de concentración aproximada $25 \mu \mathrm{M}$ y concentraciones de $\mathrm{KI}\left(\left[\mathrm{I}^{-}\right]\right)$de entre 0,1 y $10 \mathrm{mM}$. Como se puede observar en la Figura 10.1, ambos compuestos presentan una desactivación moderada, en el rango de [I-] utilizado, mostrando una disminución de la fluorescencia menor al 50 \% cuando la [I-] alcanzó un valor de $10 \mathrm{mM}$. 

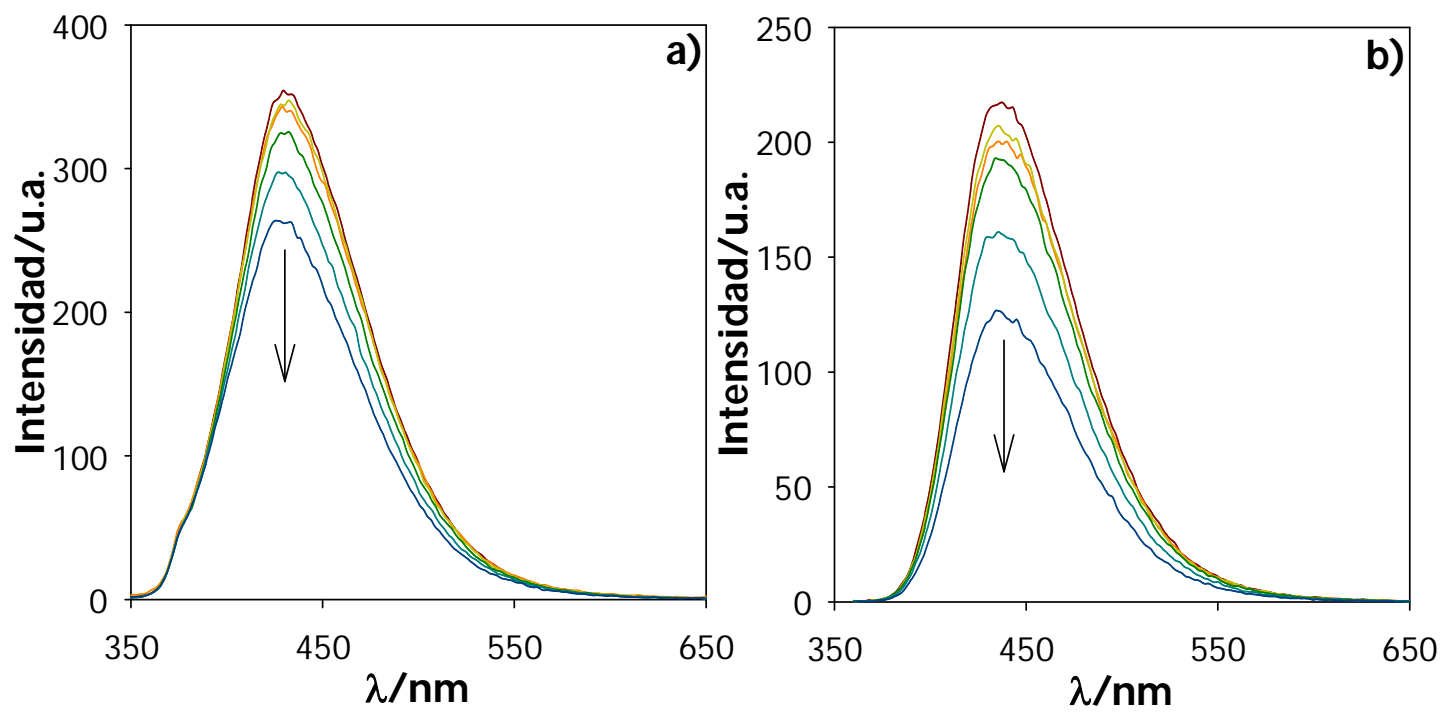

Figura 10.1 Espectros de fluorescencia de soluciones de $\mathrm{H}_{2} \mathrm{Bip}$ y Bip agregando concentraciones creciente de $K I(0,1-10 \mathrm{mM})$. a) $\mathrm{H}_{2} \operatorname{Bip}(25 \mu \mathrm{M}, \mathrm{pH}=6,8), \lambda_{\text {exc }}=335 \mathrm{~nm}$, b) $\operatorname{Bip}(21 \mu \mathrm{M}, \mathrm{pH}=6,9), \lambda_{\mathrm{exc}}=350 \mathrm{~nm}$.

Con estos resultados se realizaron los correspondientes análisis de Stern-Volmer (Figura 10.2) para $\mathrm{H}_{2}$ Bip y Bip, donde se muestra la dependencia de la intensidad de fluorescencia $\left(\mathrm{I}_{\mathrm{F}}\right)$, medida como el área total debajo de cada espectro de emisión, con la [I-]. Estos resultados muestran que concentraciones menores a $1 \mathrm{mM}$ desactivan menos del $10 \%$ del estado excitado singlete de ambos compuestos analizados. Esto significa que si se realizan fotólisis estacionarias en presencia de [I- $]<1 \mathrm{mM}$, la desactivación de los estados excitados singletes será despreciable y cualquier cambio en el comportamiento fotoquímico no podrá ser atribuido a dicho tipo de desactivación. 


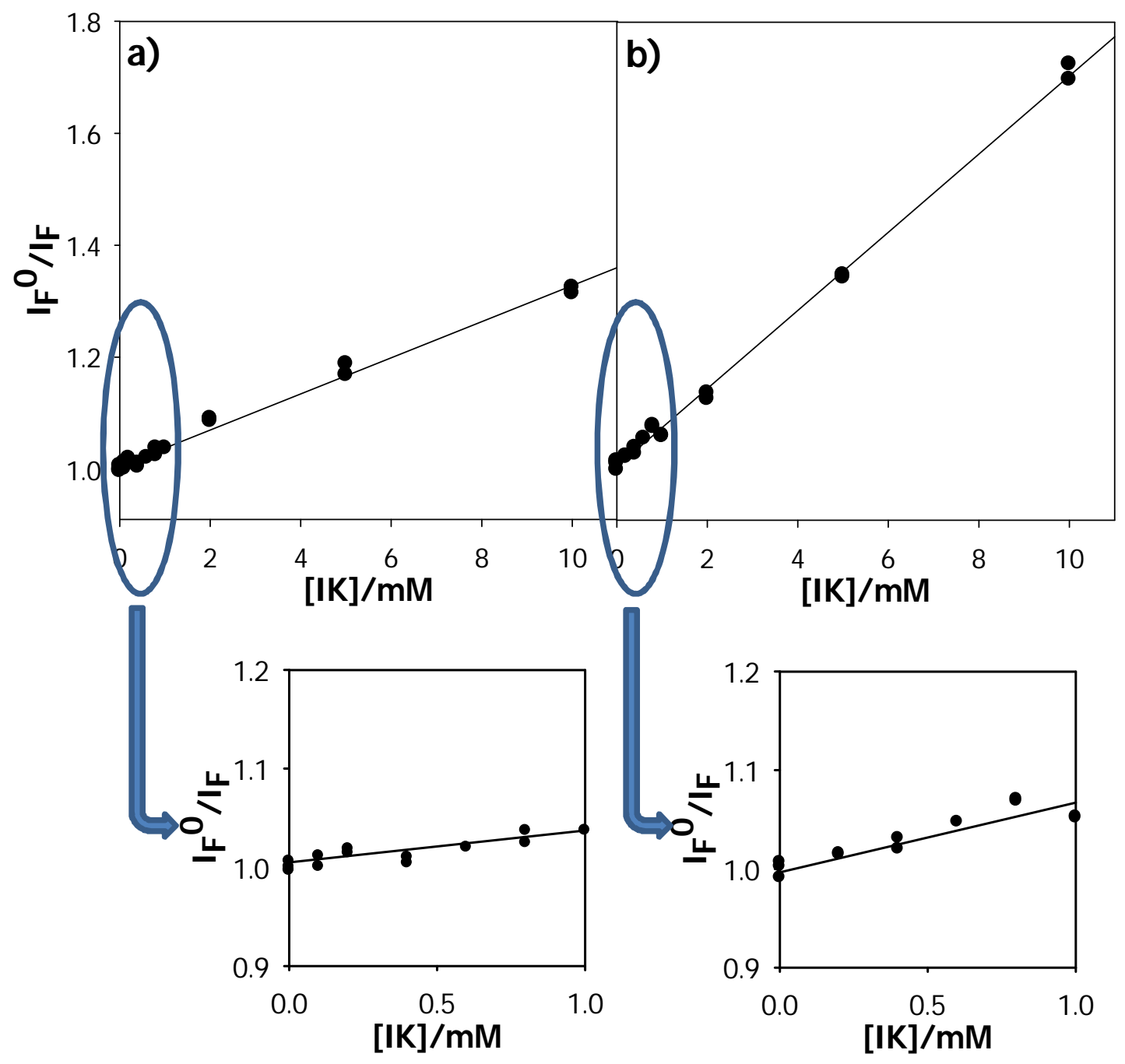

Figura 10.2 Desactivación de fluorescencia por el ión I-. Análisis de Stern-Volmer. a) $\mathrm{H}_{2}$ Bip $\left.(25 \mu \mathrm{M}, \mathrm{pH}=6,8), \lambda_{\text {exc }}=335 \mathrm{~nm}, \mathrm{~b}\right) \operatorname{Bip}(21 \mu \mathrm{M}, \mathrm{pH}=6,9), \lambda_{\text {exc }}=350 \mathrm{~nm}$.

A la luz de estas consideraciones, se realizaron experimentos de fotólisis comparativa de $\mathrm{H}_{2}$ Bip $(115 \mu \mathrm{M})$ en presencia y ausencia del ión $\mathrm{I}^{-}(600 \mu \mathrm{M})$. En la Figura 10.3 se muestra que, el I- no afecta la velocidad de formación de los dímeros. Por el contrario, la velocidad de formación de Bip es prácticamente despreciable en presencia de I- lo cual se traduce lógicamente en un perfil de consumo de reactivo muy diferente al del control en ausencia de I-. 


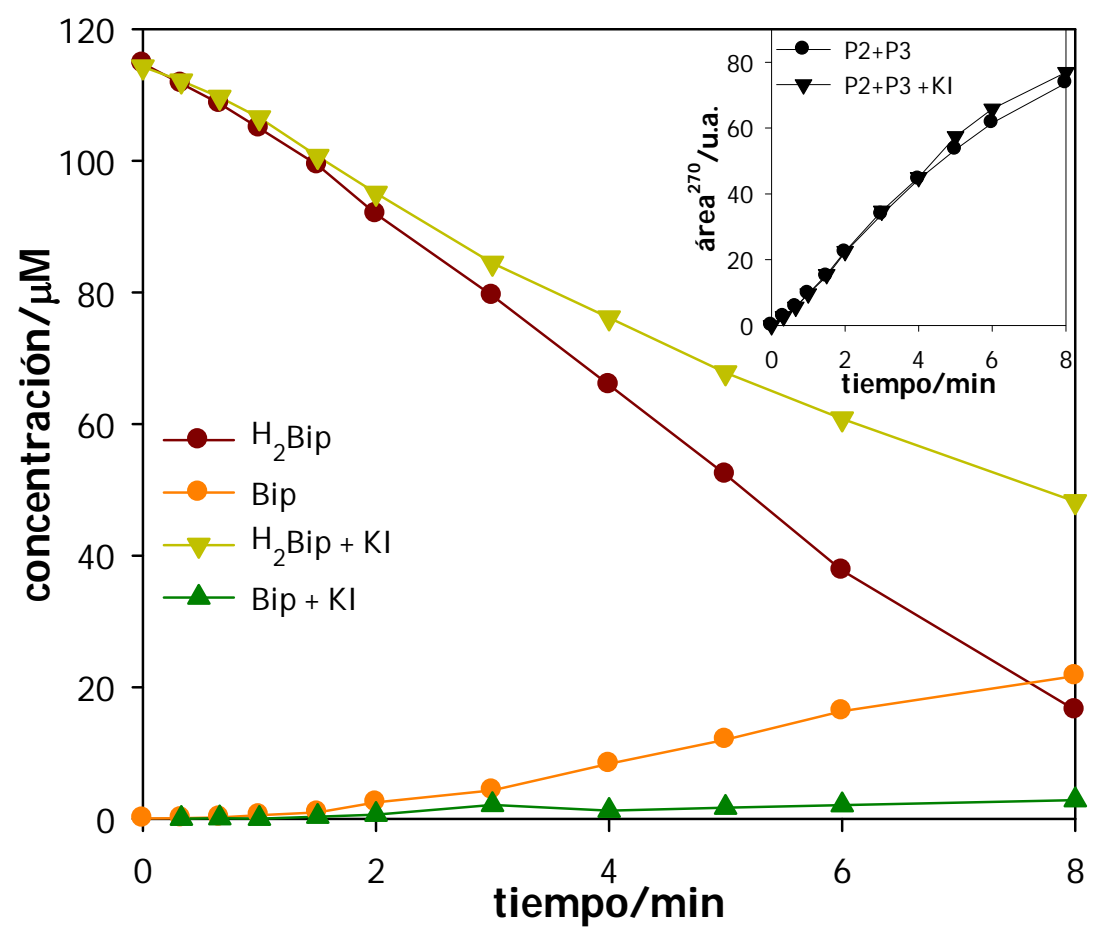

Figura 10.3 Evolución de la concentración de reactivos y productos durante la irradiación de soluciones aireadas de $\mathrm{H}_{2}$ Bip con y $\sin \mathrm{KI}\left(\left[\mathrm{H}_{2} B i p\right]_{0}=115 \mu \mathrm{M},[\mathrm{KI}]_{0}=600 \mu \mathrm{M}, \mathrm{pH}=7,0\right)$. Inset: Evolución en función del tiempo de irradiación de las áreas de P2+P3. Sistema de irradiación I y Equipo HPLC I.

El comportamiento observado en este caso es muy similar al obtenido en los experimentos realizados con soluciones saturadas de $\mathrm{O}_{2}$ (Figura 9.12). Teniendo en cuenta esta similitud en el comportamiento, además de la independencia de la reacción con el $\mathrm{O}_{2}$ observada para $\mathrm{H}_{2} \mathrm{Nep}$ (Capítulo 8), se sugiere que los dímeros se forman a partir del estado excitado singlete de $\mathrm{H}_{2} \mathrm{Bip}\left({ }^{1} \mathrm{H}_{2} \mathrm{Bip} *\right)$. Por otro lado, aunque es muy difícil detectar el efecto del ión I- en la primera parte de la oxidación de $\mathrm{H}_{2} \mathrm{Bip}$, es evidente que el $\mathrm{I}^{-}$, en las concentraciones utilizadas, inhibe completamente el efecto auto-fotocatalítico, impidiendo la aceleración del consumo de $\mathrm{H}_{2} \mathrm{Bip} \mathrm{y}$, por ende, la formación de Bip. Este efecto podría atribuirse a una desactivación selectiva del estado excitado triplete de Bip ( ${ }^{3}$ Bip*), especie que sería responsable de la oxidación fotosensibilizada de $\mathrm{H}_{2}$ Bip por Bip.

\subsection{Irradiación de mezclas $\mathrm{H}_{2}$ Bip y Bip}

En busca de mayor evidencia en favor del proceso fotosensibilizado que sufre $\mathrm{H}_{2}$ Bip cuando se acumula Bip en la solución, se decidió realizar experimentos partiendo de soluciones mezcla de $\mathrm{H}_{2}$ Bip y Bip en distintas concentraciones iniciales, e irradiando a diferentes longitudes de onda. Primero se controló que la mezcla fuera estable en ausencia de irradiación. Como no se observó ningún cambio en los espectros de 
absorción después de varias horas, se concluyó que el proceso mencionado en el capítulo anterior es fotoquímico, es decir, que se necesita el estado excitado de Bip para generar una reacción. Por lo tanto, se realizaron diferentes ensayos con el objeto de investigar este proceso en más detalle.

En una primera serie de experimentos, las soluciones fueron irradiadas con el Sistema de irradiación I. Como se observa en la Figura 10.4, se comenzó con concentraciones equimolares de ambas pterinas $(65 \mu \mathrm{M})$. A tiempos cortos de irradiación se observó un aumento en la concentración de Bip debido a una rápida oxidación del dihiroderivado. Sin embargo, al consumirse por completo $\mathrm{H}_{2} \mathrm{Bip}$, la reacción global comienza a ser dominada por la oxidación de Bip, y su concentración comienza a disminuir. Comparando la velocidad inicial de consumo de $\mathrm{H}_{2}$ Bip observada experimentalmente $\left(\left(-\mathrm{d}\left[\mathrm{H}_{2} \mathrm{Bip}\right] / \mathrm{dt}\right)_{0}=(11 \pm 1) \mu \mathrm{M} / \mathrm{min}\right)$ con la velocidad calculada a partir del rendimiento cuántico inicial de consumo de $\mathrm{H}_{2}$ Bip determinado en ausencia de $\operatorname{Bip}\left(\left(-\mathrm{d}\left[\mathrm{H}_{2} \mathrm{Bip}\right] / \mathrm{dt}\right)_{\text {cal }}=\Phi_{\mathrm{H} 2 \mathrm{Bip}} \mathrm{q}_{\mathrm{p}, \lambda}^{\mathrm{v}}=(4,6 \pm 0,9) \mu \mathrm{M} / \mathrm{min}\right)$, se observó que la velocidad experimental fue claramente mayor que la calculada. Por otro lado, la velocidad de oxidación de $\mathrm{H}_{2}$ Bip definida como d([Bip]+[Fop]+[Cap])/dt $)_{0}((7,2 \pm 0,6) \mu \mathrm{M} / \mathrm{min})$ fue del mismo orden de magnitud que $\left(-\mathrm{d}\left[\mathrm{H}_{2} \mathrm{Bip}\right] / \mathrm{dt}\right)_{0}$, en vez de ser despreciable como en el caso de los experimentos llevados a cabo con soluciones de $\mathrm{H}_{2} \mathrm{Bip}$ sin Bip inicial (Capítulo 9, Figura 9.6).

Asimismo, la proporción inicial de $\mathrm{H}_{2}$ Bip convertida en los productos P2 y P3, evaluada a partir de las áreas de los picos correspondientes, fue menor que la registrada previamente en experimentos sin adición de Bip al inicio del ensayo. En este sentido, del consumo total de $\mathrm{H}_{2} \mathrm{Bip} \sim 70 \%$ fue hacia la vía oxidativa y solo $\sim 30 \%$ fue a la formación de dímeros. También se detectó la producción de $\mathrm{H}_{2} \mathrm{O}_{2}$ que, como se muestra en la Figura10.4.c. 

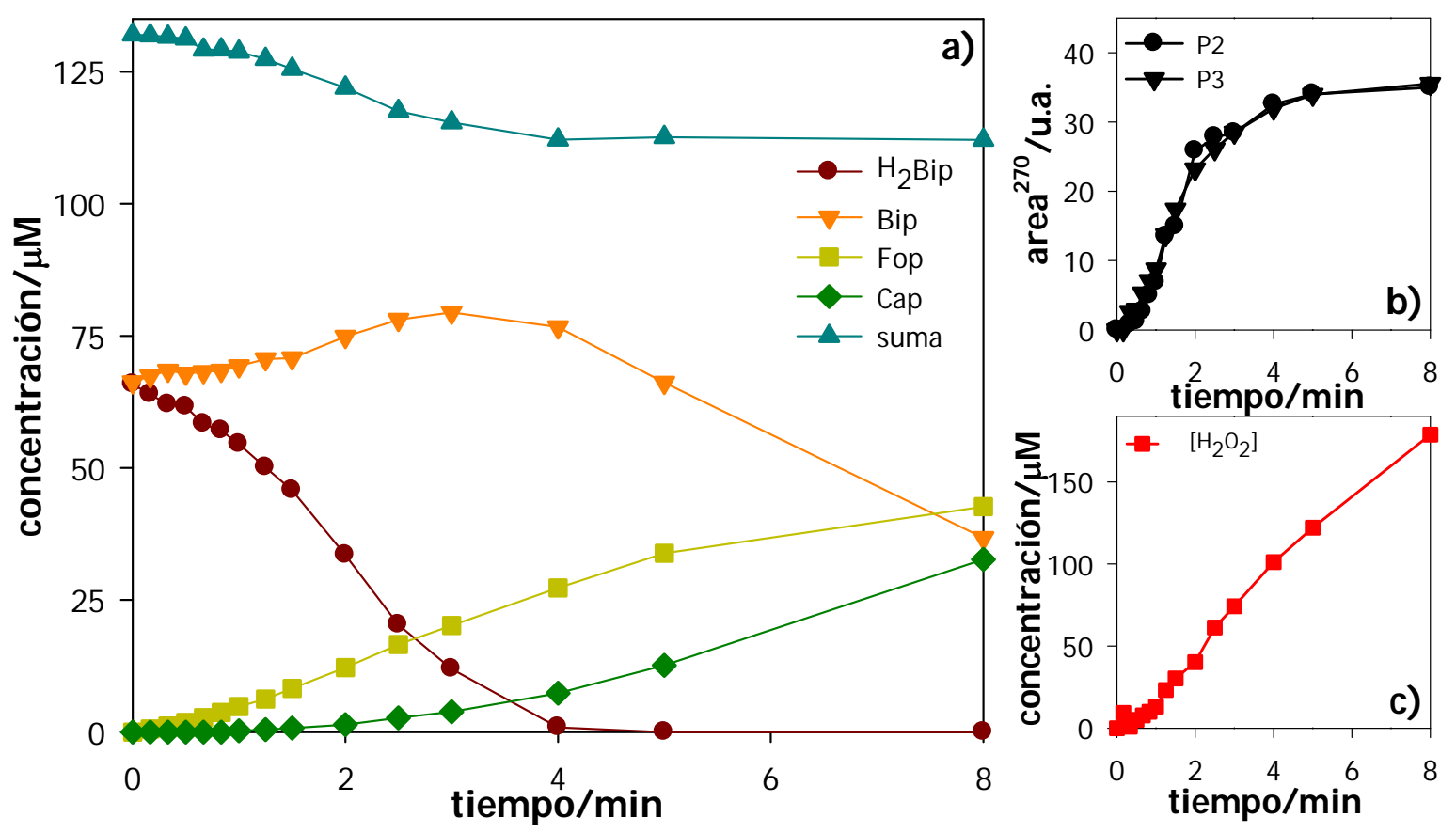

Figura 10.4 Perfil de concentración de reactivos y productos durante la irradiación de una solución aireada de $\mathrm{H}_{2} \mathrm{Bip}$ y $\mathrm{Bip}\left(\left[\mathrm{H}_{2} \mathrm{Bip}\right]_{0}=65 \mu \mathrm{M}\right.$, $\left.[\mathrm{Bip}]_{0}=65 \mu \mathrm{M} ; \mathrm{pH}=7,1\right)$. Suma, corresponde a la suma de concentraciones de $\mathrm{H}_{2}$ Bip, Bip, Fop y Cap a cada tiempo de irradiación. Sistema de irradiación I y Equipo HPLC I.

Otros experimentos fueron realizados utilizando el Sistema de irradiación II, en condiciones donde $\mathrm{H}_{2}$ Bip absorbe muy poca radiación y sólo Bip es excitada, es decir, a $\lambda_{\text {exc }}>370 \mathrm{~nm}$ (Sección 2.2). Consecuentemente, en este caso, fue posible trabajar con soluciones de $\mathrm{H}_{2}$ Bip cuya concentración inicial fue significativamente mayor a la concentración de Bip. Como se observa en la Figura 10.5.a, la proporción de $\mathrm{H}_{2} \mathrm{Bip}$ convertida en Bip fue muy elevada (> 80\%) y, por consiguiente, la cantidad de P2 y P3 encontrada fue casi indetectable. El mismo experimento, partiendo de las mismas concentraciones iniciales de $\mathrm{H}_{2} \mathrm{Bip}$ y Bip, fue realizado irradiando en ausencia de $\mathrm{O}_{2}$ y aireando la solución antes del análisis por HPLC. Como se muestra en la Figura 10.5.b, mientras que el consumo de $\mathrm{H}_{2}$ Bip es despreciable debido a que casi no absorbe radiación, Bip sufre las reacciones presentadas en el Capítulo 6, generando Fop como producto final. Por otro lado, a modo de control, se irradió una solución de $\mathrm{H}_{2} \mathrm{Bip}$ en ausencia de Bip inicial y en presencia de $\mathrm{O}_{2}$ (Figura 10.5.c). En este caso, y como era de esperar, el consumo del dihidroderivado fue muy pequeño, al igual que la generación de Bip.

Estos resultados muestran que cuando $\mathrm{H}_{2}$ Bip se encuentra en presencia de Bip y solo esta última es excitada, se produce la oxidación del dihidroderivado dando lugar a 
su vez a la formación de su análogo oxidado. Queda claro que este proceso tiene lugar aún en ausencia de radiación absorbida por $\mathrm{H}_{2}$ Bip. Esto confirma, la existencia de un proceso fotosensibilizado de $\mathrm{H}_{2}$ Bip por parte de Bip en el cual es indispensable el $\mathrm{O}_{2}$.

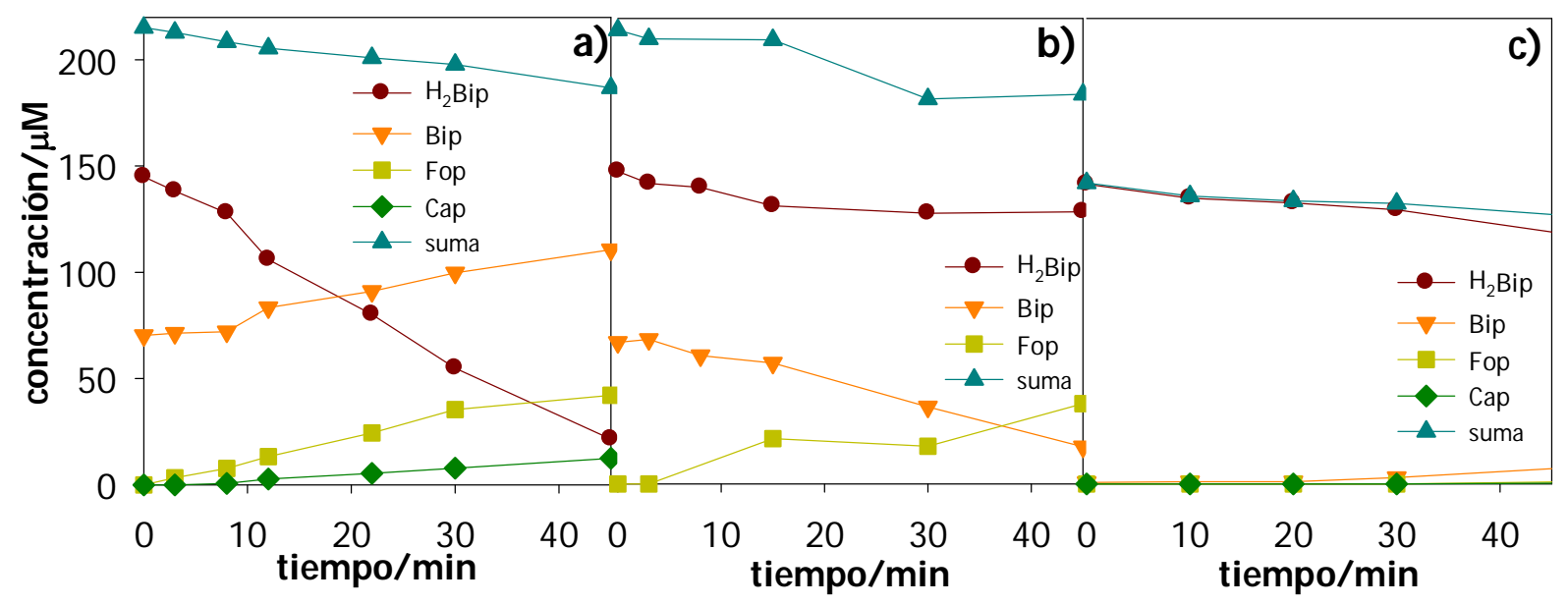

Figura 10.5 Perfiles de concentración de reactivos y productos durante la irradiación de: a) solución mezcla en presencia de $\mathrm{O}_{2}\left(\left[\mathrm{H}_{2} \mathrm{Bip}\right]_{0}=145 \mu \mathrm{M}\right.$, $\left.\left.[\mathrm{Bip}]_{0}=70 \mu \mathrm{M}, \mathrm{pH}=6,9\right) ; \mathrm{b}\right)$ solución mezcla en ausencia de $\mathrm{O}_{2}\left(\left[\mathrm{H}_{2} \mathrm{Bip}\right]_{0}=148 \mu \mathrm{M}\right.$, $\left.\left.[\mathrm{Bip}]_{0}=67 \mu \mathrm{M}, \mathrm{pH}=6,4\right) ; \mathrm{c}\right)$ solución conteniendo solo $\mathrm{H}_{2} \mathrm{Bip}\left(\left[\mathrm{H}_{2} \mathrm{Bip}\right]_{0}=142 \mu \mathrm{M}, \mathrm{pH}=7,1\right)$. Sistema de irradiación II $(380 \mathrm{~nm})$ y Equipo HPLC II.

En consecuencia, la formación de Bip a partir de $\mathrm{H}_{2}$ Bip se lleva a cabo por dos procesos. En primer término, existe probablemente una reacción inicial que se produce cuando $\mathrm{H}_{2} \mathrm{Bip}$ absorbe radiación, se excita y reacciona con $\mathrm{O}_{2}$. Esta vía, cuyo mecanismo no ha podido ser dilucidado, no necesita la presencia de Bip excitada, y sería la responsable por la pequeña formación inicial de Bip durante la irradiación de $\mathrm{H}_{2}$ Bip en aire. La segunda vía de oxidación del dihidroderivado, es un proceso fotosensibilizado donde el estado excitado triplete de Bip reacciona con $\mathrm{H}_{2} \mathrm{Bip}$ en estado basal para oxidarla y generar más Bip. En consecuencia, este proceso es el responsable de la aceleración en la velocidad de oxidación de $\mathrm{H}_{2} \mathrm{Bip}$ observada en los experimentos descriptos en el capítulo anterior, que tiene lugar cuando se irradian soluciones acuosas de $\mathrm{H}_{2} \mathrm{Bip}$ en presencia de $\mathrm{O}_{2}$. Por otro lado, también explica las altas velocidades de consumo de $\mathrm{H}_{2}$ Bip y formación de Bip observadas cuando se irradia una solución mezcla de ambas pterinas.

\subsection{Irradiación de mezclas $\mathrm{H}_{2} \mathrm{Bip}$ con otras pterinas oxidadas}

Para determinar si la fotosensibilización de $\mathrm{H}_{2} \mathrm{Bip}$ en soluciones aireadas es posible también con otros compuestos pterínicos además de Bip, se realizaron nuevos experimentos. En este caso, se trabajó con pterina $(\mathrm{Ptr})^{6}$ y 6 -metilpterina (Mep). ${ }^{7} \mathrm{Se}$ 
eligieron estos dos derivados pterínicos porque, como se explicó en el Capítulo 3, son lo suficiente fotoestables como para no degradarse durante el período de irradiación. De esta manera, se evita la generación de nuevos productos, simplificando así el análisis.

Sin embargo, en el caso de los experimentos utilizando Ptr como fotosensibilizador, el análisis fue bastante complejo. Esto se debe a que Ptr tiene un tiempo de retención en las corridas cromatográficas muy similar a Bip y $\mathrm{H}_{2} \mathrm{Bip}$, lo cual hizo muy difícil la integración de los distintos picos y la consecuente obtención de perfiles de concentración. Por este motivo, se presentará a continuación sólo el estudio llevado a cabo con Mep.

En la Figura 10.6 se muestran los resultados obtenidos del experimento de irradiación (Sistema de irradiación II, $380 \mathrm{~nm}$ ) en aire de una solución mezcla de $\mathrm{H}_{2} \mathrm{Bip}$ $(100 \mu \mathrm{M})$ y Mep $(40 \mu \mathrm{M})$. Mientras que Mep no se consume significativamente, sí se observa un gran consumo de $\mathrm{H}_{2}$ Bip, producción de Bip y mínimas cantidades de P2 y P3. Es decir que el dihidroderivado se oxida generando Bip a partir de la fotosensibilización sufrida por el estado excitado de Mep. Se realizaron distintos experimentos de control para descartar la existencia de otro proceso. Por un lado, se mezclaron soluciones de $\mathrm{H}_{2}$ Bip y Mep y se conservaron en oscuridad por un período prolongado de tiempo. En estos ensayos no se observó cambio alguno en los espectros de absorción. Otro control se realizó irradiando $\mathrm{H}_{2}$ Bip a $380 \mathrm{~nm}$ en ausencia de Mep y no se detectaron cantidades apreciables de Bip (similar al experimento representado en la Figura 10.5.c).

Además, se realizaron experimentos adicionales utilizando el Sistema de irradiación I (350 nm). En este caso $\mathrm{H}_{2}$ Bip absorbe más radiación, motivo por el cual se trabajo con una relación de concentraciones inversa a la del experimento anterior, es decir, $\mathrm{H}_{2}$ Bip $(56 \mu \mathrm{M})$ en menor concentración que Mep $(90 \mu \mathrm{M})$. Como se observa en la Figura 10.7, los resultados son muy similares a los obtenidos irradiando la solución mezcla a $380 \mathrm{~nm}$. Es importante mencionar que se observa una mayor generación de productos P2 y P3 debido a que, como se dijo anteriormente, en este caso $\mathrm{H}_{2} \mathrm{Bip}$ presenta una mayor capacidad de absorción de la radiación incidente. 


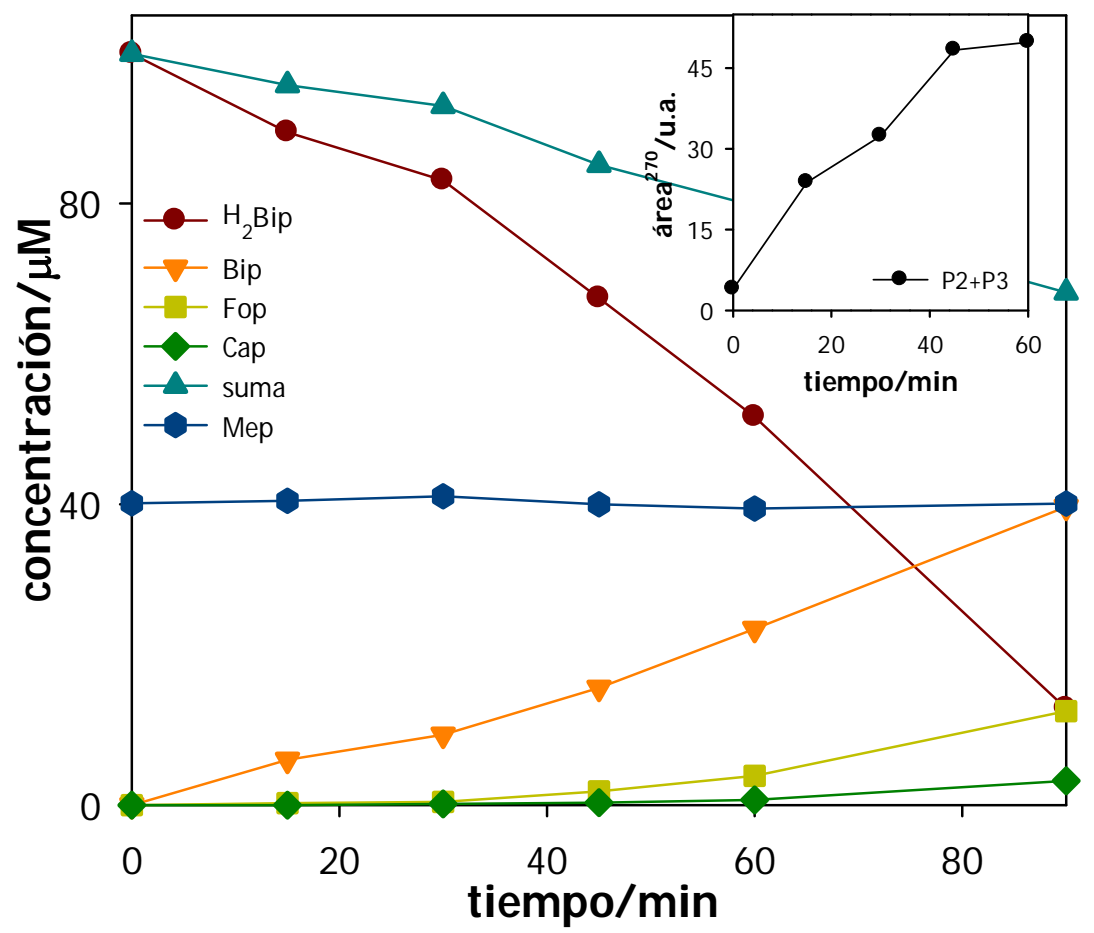

Figura 10.6 Perfiles de concentración de reactivos y productos durante la irradiación, partiendo de una solución mezcla de $\mathrm{H}_{2} \mathrm{Bip}$ y $\mathrm{Mep}\left(\left[\mathrm{H}_{2} \mathrm{Bip}\right]_{0}=100 \mu \mathrm{M}\right.$, $[\mathrm{Mep}]_{0}=40 \mu \mathrm{M}, \mathrm{pH}$ = 6,9). Suma, corresponde a la suma de concentraciones de $\mathrm{H}_{2} \mathrm{Bip}$, Bip, Fop y Cap a cada tiempo de irradiación. Sistema de irradiación II y Equipo HPLC II. Inset: evolución en el tiempo de irradiación del área total de P2+P3.

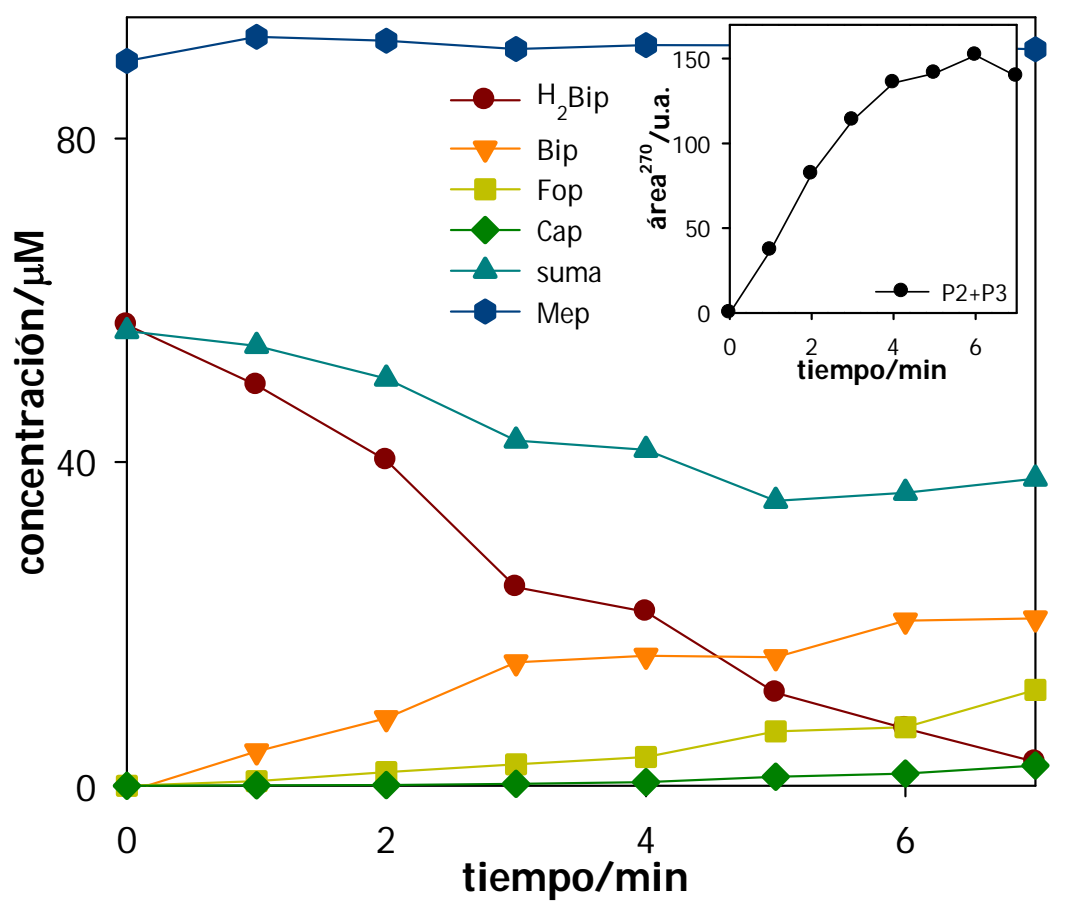

Figura 10.7 Perfiles de concentración de reactivos y productos durante la irradiación, partiendo de una solución mezcla de $\mathrm{H}_{2} \mathrm{Bip}$ y $\mathrm{Mep}\left(\left[\mathrm{H}_{2} \mathrm{Bip}\right]_{0}=56 \mu \mathrm{M},[\mathrm{Mep}]_{0}=90 \mu \mathrm{M}, \mathrm{pH}=\right.$ $7,1)$. Suma, corresponde a la suma de concentraciones de $\mathrm{H}_{2} \mathrm{Bip}$, Bip, Fop y Cap a cada tiempo de irradiación. Sistema de irradiación I y Equipo HPLC I. Inset: evolución en el tiempo de irradiación del área total de P2+P3. 
Por lo tanto, los resultados observados usando diferentes compuestos pterínicos como fotosensibilizadores, demuestran que la conversión fotosensibilizada de $\mathrm{H}_{2} \mathrm{Bip}$ a Bip tiene lugar no solo con Bip como sensibilizador, sino también con Mep, Ptr y, probablemente, con cualquier otra pterina oxidada capaz de absorber radiación a la longitud de onda de excitación y generar estados excitados.

\subsection{Análisis del mecanismo de reacción}

En la primera sección se postuló la participación del 3Bip*en la vía oxidativa de $\mathrm{H}_{2}$ Bip. Sin embargo, sabiendo que Bip y sus fotoproductos (Fop y Cap) generan, eficientemente, ${ }^{1} \mathrm{O}_{2}$ bajo irradiación UV-A (Capítulo 3) y que, a su vez, las dihidropterinas reaccionan con esta especie reactiva de oxígeno (Dántola et al.) ${ }^{9}$, es necesario evaluar la hipótesis que involucra al ${ }^{1} \mathrm{O}_{2}$ como intermediario en la reacción 0 proceso fotosensibilizado (Tipo II). Esta hipótesis es compatible con los resultados presentados hasta el momento ya que el aumento en la concentración de Bip al avanzar la fotólisis provocaría un aumento en la concentración de ${ }^{1} \mathrm{O}_{2}$ con el consiguiente aumento en la velocidad de la fotooxidación de $\mathrm{H}_{2}$ Bip a Bip.

Para tal fin se recurrió a cálculos cinéticos y balances de masa y estequiométricos. Dántola et al. demostraron que $\mathrm{H}_{2} \mathrm{Bip}$ es fácilmente oxidada por ${ }^{1} \mathrm{O}_{2}$ generando como productos Bip y $\mathrm{H}_{2} \mathrm{O}_{2}{ }^{9}$ En dicho trabajo se determinó que en la reacción de oxidación de $\mathrm{H}_{2}$ Bip por ${ }^{1} \mathrm{O}_{2}$ la fracción de reactivo convertida en Bip ( $\Delta[\mathrm{Bip}] / \Delta\left[\mathrm{H}_{2} \mathrm{Bip}\right]$ ) es 0,42 $\pm 0,03$. Asimismo, encontraron que la relación entre $\mathrm{H}_{2} \mathrm{O}_{2}$ producida y $\mathrm{H}_{2}$ Bip consumida $\left(\Delta\left[\mathrm{H}_{2} \mathrm{O}_{2}\right] / \Delta\left[\mathrm{H}_{2} \mathrm{Bip}\right]\right)$ es igual a $0,17 \pm 0,03$.

Los valores obtenidos por Dántola et al. fueron comparados con los obtenidos en las reacciones fotoquímicas de $\mathrm{H}_{2}$ Bip descriptas en el capítulo anterior. Realizando un balance de masa de la fotooxidación, se observó que la relación $\Delta\left[\mathrm{H}_{2} \mathrm{O}_{2}\right] / \Delta\left[\mathrm{H}_{2} \mathrm{Bip}\right]$ (siendo $\Delta\left[\mathrm{H}_{2} \mathrm{O}_{2}\right]=\left[\mathrm{H}_{2} \mathrm{O}_{2}\right]-\left[\right.$ Fop]-2[Cap] y $\Delta\left[\mathrm{H}_{2} \mathrm{Bip}\right]=[\mathrm{Bip}]+[$ Fop] $+[$ Cap $]$ ) es muy cercano a 1 para experimentos realizados tanto en aire como en atmósfera saturada en $\mathrm{O}_{2}$. Asimismo, los cálculos de $\Delta[\mathrm{Bip}] / \Delta\left[\mathrm{H}_{2} \mathrm{Bip}\right]$ en la reacción fotoquímica dan valores más elevados que el valor 0,42 mencionado previamente para la reacción frente a ${ }^{1} \mathrm{O}_{2}$. Esta diferencia se aprecia mejor en experimentos donde el consumo de $\mathrm{H}_{2} \mathrm{Bip}$ por la vía oxidativa es mucho mayor que por la vía que genera los productos diméricos. 
Particularmente, en los experimentos de las secciones anteriores, donde se parte de una solución mezcla de $\mathrm{H}_{2}$ Bip con una pterina oxidada.

En este caso, cuando Bip absorbe radiación, genera el 1Bip*, que por entrecruzamiento de sistemas pasa a ${ }^{3} \mathrm{Bip} *$ (reacción 1). Éste reacciona con $\mathrm{O}_{2}$ para generar ${ }^{1} \mathrm{O}_{2}$ (reacción 2). Por otro lado, la desactivación de ${ }^{1} \mathrm{O}_{2}$ puede ocurrir en forma no radiativa (por transferencia de energía al solvente, reacción 3) o radiativa (emitiendo radiación, reacción 4). Generalmente, el rendimiento cuántico de fosforescencia es despreciable respecto del rendimiento cuántico de desactivación por el solvente $\left(k_{d} \gg\right.$ $\mathrm{k}_{\mathrm{e}}$ ). Además, al estar en presencia de Bip y $\mathrm{H}_{2} \mathrm{Bip}$, existen también las reacciones de quenching físico y químico (reacciones 5-7).

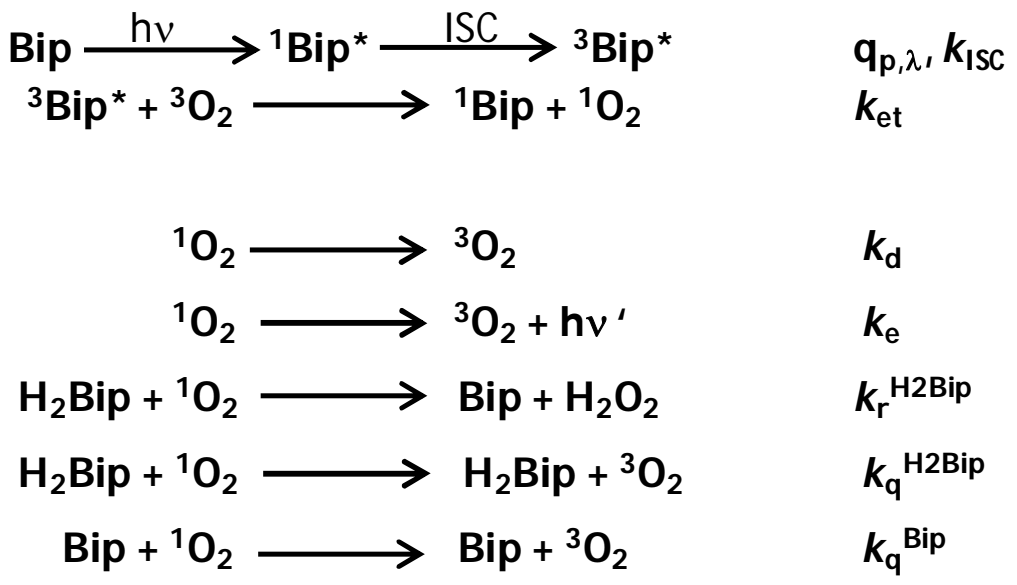

donde $\mathrm{q}_{\mathrm{p}, \lambda}$ es la intensidad de la radiación absorbida por Bip (einstein $\mathrm{L}^{-1} \mathrm{~S}^{-1}$ ); $\mathrm{k}_{\mathrm{ISC}}$ es la constante de entrecruzamiento de sistemas; $k_{e t}$ es la constante de velocidad de transferencia de energía; $\mathrm{k}_{\mathrm{d}} \mathrm{y} \mathrm{k}_{\mathrm{e}}$ son las constantes de velocidad de desactivación no radiativa y radiativa de ${ }^{1} \mathrm{O}_{2}$, respectivamente; $\mathrm{k}_{\mathrm{q}}$ es la constante de velocidad de quenching físico; $\mathrm{k}_{\mathrm{r}}$ es la constante de velocidad de reacción de oxidación de $\mathrm{H}_{2}$ Bip por ${ }^{1} \mathrm{O}_{2}$ (quenching químico).

En condiciones de irradiación continua, teniendo en cuenta las reacciones planteadas, se puede escribir la siguiente expresión:

$$
\frac{d\left[{ }^{1} O_{2}\right]}{d t}=q_{p, \lambda}{ }^{B i p} \Phi_{\Delta}^{B i p}-k_{d}\left[{ }^{1} O_{2}\right]-k_{e}\left[{ }^{1} O_{2}\right]-\left(k_{q}{ }^{H 2 B i p}+k_{r}{ }^{H 2 B i p}\right)\left[H_{2} B i p\right]\left[{ }^{1} O_{2}\right]-k_{q}{ }^{\text {Bip }}[\operatorname{Bip}]\left[{ }^{1} O_{2}\right]
$$

donde $\Phi_{\Delta}$ Bip es el rendimiento cuántico de formación de ${ }^{1} \mathrm{O}_{2} \mathrm{y}\left(\mathrm{k}_{\mathrm{r}}+\mathrm{k}_{\mathrm{q}}\right)=\mathrm{k}_{\mathrm{t}}$ : constante de velocidad de quenching total. Teniendo en cuenta la aproximación al estado estacionario, puede suponerse que $\mathrm{d}\left[{ }^{1} \mathrm{O}_{2}\right] / \mathrm{dt} \approx 0$ : 


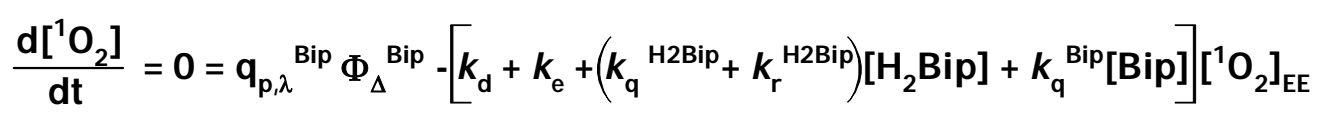

Reordenando la ecuación 9 y despreciando $\mathrm{k}_{\mathrm{e}}$, que es mucho menor que $\mathrm{k}_{\mathrm{d}}{ }^{10} \mathrm{se}$ obtiene la siguiente expresión para la concentración de estado estacionario de ${ }^{1} \mathrm{O}_{2}$ :

$$
\left[{ }^{1} \mathbf{O}_{2}\right]_{\mathrm{EE}}=\frac{\mathbf{q}_{\mathrm{p}, \lambda}{ }^{\mathrm{Bip}} \Phi_{\Delta}^{\text {Bip }}}{k_{\mathrm{d}}+\mathrm{k}_{\mathrm{t}}^{\mathrm{H} \text { Bip }}\left[\mathrm{H}_{2} \text { Bip }\right]+\mathrm{k}_{\mathrm{q}}^{\text {Bip }}[\mathrm{Bip}]}
$$

Sabiendo que la velocidad de consumo de de $\mathrm{H}_{2}$ Bip por reacción con ${ }^{1} \mathrm{O}_{2}$ está dada por:

$$
\left.-\left(\frac{\mathrm{d}\left[\mathrm{H}_{2} \mathrm{Bip}\right]}{\mathrm{dt}}\right)=\mathrm{k}_{\mathrm{r}} \mathrm{l}^{1} \mathbf{O}_{2}\right]\left[\mathrm{H}_{2} \mathrm{Bip}\right]
$$

Reemplazando $\left[{ }^{1} \mathrm{O}_{2}\right]$ por la expresión de la ecuación 10 , se obtiene:

$$
-\left(\frac{\mathrm{d}\left[\mathrm{H}_{2} \mathrm{Bip}\right]}{\mathrm{dt}}\right)=\frac{\mathrm{q}_{\mathrm{p}, \lambda}^{{ }^{\text {Bip }}} \Phi_{\Delta}^{\text {Bip }} \mathbf{k}_{\mathrm{r}}\left[\mathrm{H}_{2} \text { Bip }\right]}{\mathrm{k}_{\mathrm{d}}+\mathrm{k}_{\mathrm{t}}^{\text {H2Bip }}\left[\mathrm{H}_{2} \text { Bip }\right]+\mathrm{k}_{\mathrm{q}}^{\text {Bip }}[\mathrm{Bip}]}
$$

Conociendo los valores de las distintas constantes y $\Phi_{\Delta}$ Bip detallados en el Capítulo 3, se puede calcular una velocidad de consumo de $\mathrm{H}_{2}$ Bip de la reacción entre $\mathrm{H}_{2}$ Bip y ${ }^{1} \mathrm{O}_{2}$, para cada par de concentraciones inicial de $\mathrm{H}_{2} \mathrm{Bip}$ y Bip. Este cálculo se

\begin{tabular}{|c|c|c|c|c|c|}
\hline Experimento & {$\left[\mathrm{H}_{2} \mathrm{Bip}\right] / \mu \mathrm{M}$} & {$[\mathrm{Bip}] / \mu \mathrm{M}$} & {$[\mathrm{Mep}] / \mu \mathrm{M}$} & $\left(\mathrm{d}\left[\mathrm{H}_{2} \mathrm{Bip}\right] / \mathrm{dt}\right)_{\Delta}$ & $\left.\left(\mathrm{d}\left[\mathrm{H}_{2} \mathrm{Bip}\right] / \mathrm{dt}\right)_{0}\right)^{\exp }$ \\
\hline I & 105,7 & 15,5 & - & 0,024 & 0,24 \\
\hline II & 67,7 & 63,2 & - & 0,059 & 0,20 \\
\hline III & 146,0 & 69,0 & - & 0,009 & 0,047 \\
\hline IV & 100,0 & - & 40,0 & 0,004 & 0,013 \\
\hline
\end{tabular}
realizó para varios experimentos y se comparó con la velocidad experimental obtenida a partir de los perfiles de concentración obtenidos por HPLC. Los resultados se muestran en la Tabla 10.1.

Tabla 10.1 Valores de velocidad inicial de consumo de $\mathrm{H}_{2} B i p$ experimentales $\left(\left(\mathrm{d}\left[\mathrm{H}_{2} \mathrm{Bip}\right] / \mathrm{dt}\right)_{0}{ }^{\mathrm{exp}}\right)$ y de velocidades calculadas de la reacción entre $\mathrm{H}_{2} \mathrm{Bip}$ y ${ }^{1} \mathrm{O}_{2}$. En los experimentos I y II se utilizó el Sistema de irradiación I $\left(\lambda_{\text {exc }}=350 \mathrm{~nm}\right)$ y en los experimentos III y IV, el Sistema de irradiación II $\left(\lambda_{\text {exc }}=380 \mathrm{~nm}\right)$.

De los valores observados en la tabla se desprende que las velocidades calculadas son mucho menores que las velocidades obtenidas experimentalmente. Por lo tanto, si bien puede existir una contribución de la reacción entre ${ }^{1} \mathrm{O}_{2}$ y $\mathrm{H}_{2} \mathrm{Bip}$ al consumo general 
de $\mathrm{H}_{2} \mathrm{Bip}$, ciertamente esta vía no es significativa. $\mathrm{Y}$, sin lugar a dudas, no es responsable de la aceleración en la velocidad de consumo de $\mathrm{H}_{2} \mathrm{Bip}$ observada.

Por lo tanto, la oxidación de $\mathrm{H}_{2}$ Bip puede ser fotoinducida por transferencia de energía o de electrones desde ${ }^{3} \mathrm{Bip} *$. Si fuera mediante el primer mecanismo, se generaría el ${ }^{3} \mathrm{H}_{2} \mathrm{Bip} *$ (reacción 13) que luego reaccionaría con $\mathrm{O}_{2}$ para dar $\mathrm{Bip}$ y $\mathrm{H}_{2} \mathrm{O}_{2}$ (reacción 14). Sin embargo, una transferencia de energía desde estados excitados de Bip a $\mathrm{H}_{2}$ Bip parece poco probable, ya que, de acuerdo a sus espectros de absorción, las transiciones electrónicas de $\mathrm{H}_{2}$ Bip involucran mayor energía que las de su análogo oxidado. Por ende, en principio una transferencia de energía no sería termodinámicamente favorable.

Por el contrario, si el mecanismo fuera por transferencia de electrones, se transferiría un electrón desde el estado basal de $\mathrm{H}_{2} \mathrm{Bip}$ al estado ${ }^{3} \mathrm{Bip} *$ (reacción 15). Ya se ha demostrado la capacidad de ciertas pterinas oxidadas para actuar como fotosensibilizadores por transferencia de electrones, por ejemplo, en el caso de oxidación de nucleótidos por pterina. ${ }^{11,12}$ En este caso, el fotosensibilizador en su estado excitado triplete reaccionaría con el dihidroderivado para dar el radical anión correspondiente al derivado pterínico oxidado (Bip ${ }^{\bullet-}$ y el catión radical del dihidroderivado $\left(\mathrm{H}_{2} \mathrm{Bip}^{\bullet+}\right)$. Luego, Bip ${ }^{\bullet-}$ es capaz de reaccionar con $\mathrm{O}_{2}$ para generar anión superóxido $\left(\mathrm{O}_{2}{ }^{\bullet-}\right.$, reacción 16) y $\mathrm{H}_{2} \mathrm{Bip}{ }^{\bullet+}$ se oxida produciendo Bip (reacción 17). Asimismo, se genera $\mathrm{H}_{2} \mathrm{O}_{2}$ (reacciones 18-19).

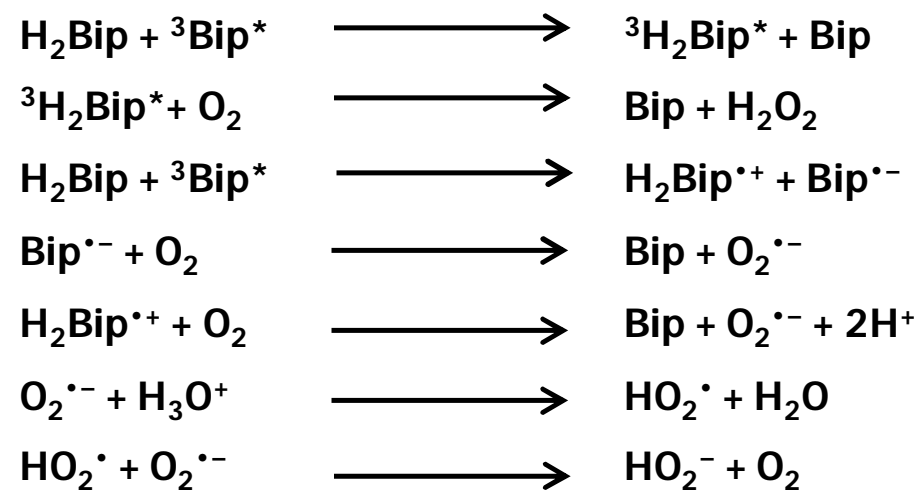

\subsection{Investigación de la oxidación fotosensibilizada de $\mathrm{H}_{2} \mathrm{Nep}$}

Como se mencionó anteriormente, existe una gran diferencia de reactividad fotoquímica entre $\mathrm{H}_{2}$ Bip y $\mathrm{H}_{2} \mathrm{Nep}$. Mientras que la primera posee una vía oxidativa importante generando su análogo oxidado, la cual compite con la vía de dimerización (Sección 9.2), $\mathrm{H}_{2} \mathrm{Nep}$ sólo genera dímeros independientemente de la concentración de 
O2. Sin embargo, que no produzca Nep no significa que no pueda ser fotosensibilizada por las pterinas aromáticas, motivo por el cual se decidió investigar la existencia de un proceso fotosensibilizado también para el caso de $\mathrm{H}_{2} \mathrm{Nep}$.

Para esto se realizaron distintos experimentos partiendo de mezclas de $\mathrm{H}_{2} \mathrm{Nep}$ y Nep en presencia de $\mathrm{O}_{2}$, utilizando el Sistema de irradiación I. En la Figura 10.8 se muestran los perfiles de concentración y áreas con respecto al tiempo de irradiación, para una mezcla de $\mathrm{H}_{2} \mathrm{Nep}$ y Nep (Figura 10.8.a). Con fines comparativos también se muestran los resultados obtenidos al irradiar una solución conteniendo, únicamente, $\mathrm{H}_{2} \mathrm{Nep}$ (Figura 10.8.b). Principalmente, se aprecia que la velocidad de consumo del dihidroderivado se ve incrementada con respecto a la velocidad de consumo de $\mathrm{H}_{2} \mathrm{Nep}$ irradiada en ausencia de Nep inicial. Además, se detecta la generación de Fop y pequeñas cantidades de Cap, ya que estos son los productos de la fotólisis de Nep (Capítulo 6) y Fop, ${ }^{13}$ respectivamente. Es necesario destacar que la cantidad total de pterinas oxidadas ([Nep]+[Fop]+[Cap]) aumenta con el tiempo de irradiación (Figura 10.8.a). Más aún, la cantidad de Fop y Cap generada a cada tiempo de irradiación es mayor que la concentración de Nep consumida. Por ejemplo, a ti $=4 \mathrm{~min}$ la concentración de Fop + Cap es $30 \mu \mathrm{M}$, mientras que la concentración de Nep consumida es $15 \mu \mathrm{M}$. Es decir que también se está generando algo de Nep a partir de $\mathrm{H}_{2} \mathrm{Nep}$ en el proceso fotosensibilizado.

Por otro lado, también se observa una mayor formación del compuesto X (ver descripción de este producto en Sección 8.1), cuando se parte de una solución mezcla de $\mathrm{H}_{2}$ Nep y Nep. P2 y P3 en cambio, sufren una disminución en su velocidad de formación, cuando se comparan con la solución de $\mathrm{H}_{2} \mathrm{Nep}$. Esto demuestra que, $\mathrm{H}_{2} \mathrm{Nep}$ sufre un proceso fotosensibilizado a partir de Nep. En este proceso, alrededor de un $10 \%$ del reactivo inicial se oxida generando Nep, y otra parte produce el compuesto X. 


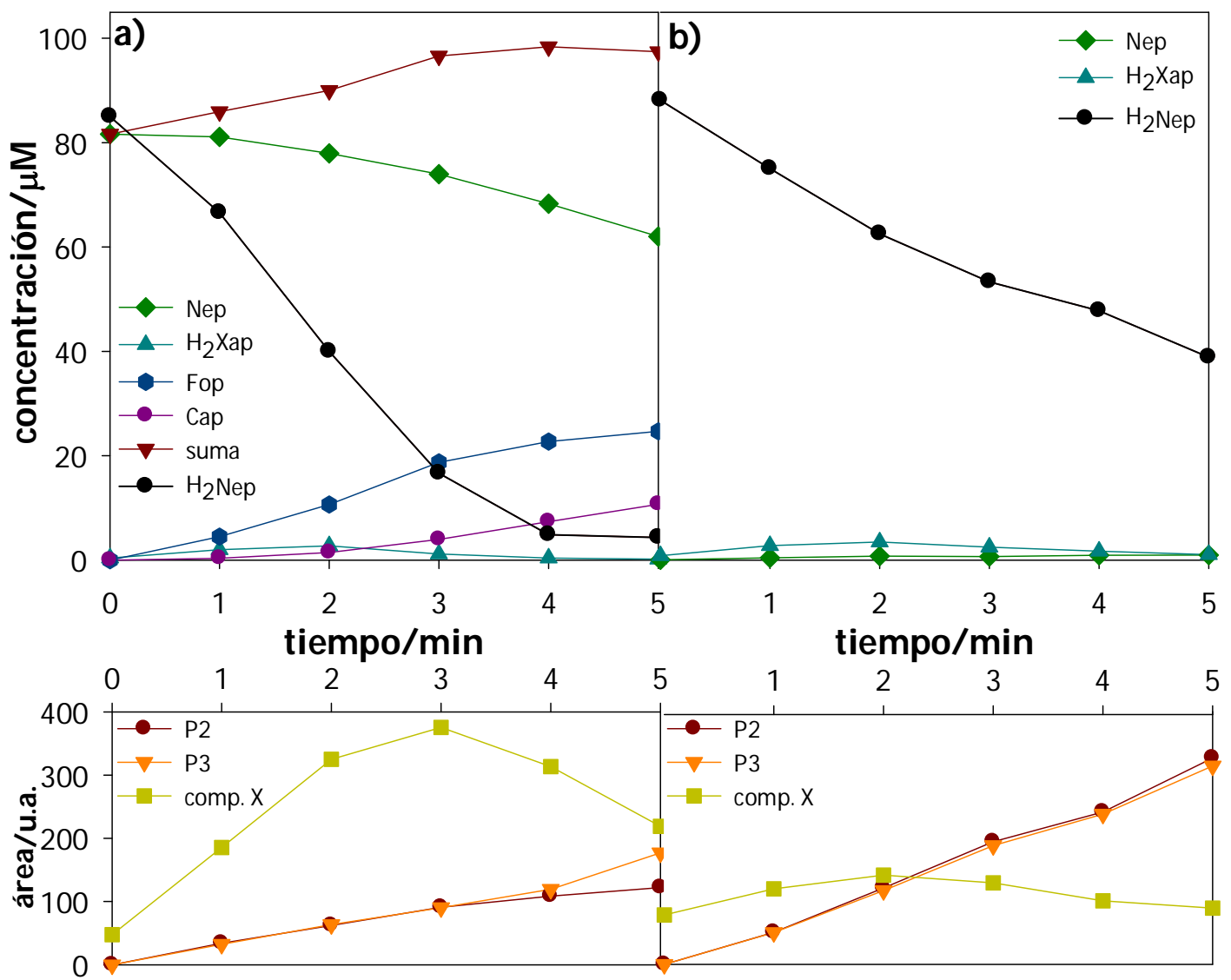

Figura 10.8 Perfiles de concentración de reactivos y productos en función del tiempo de irradiación. Sistema de irradiación I y Equipo HPLC I. a) $\left[\mathrm{H}_{2} \mathrm{Nep}\right]_{0}=85 \mu \mathrm{M}$, $[\mathrm{Nep}]_{0}=82 \mu \mathrm{M}$, $\mathrm{pH}=7,2, \mathrm{~b}) \mathrm{H}_{2} \mathrm{Nep}=88 \mu \mathrm{M}, \mathrm{pH}=7,0$. Suma, corresponde a la suma de concentraciones de Nep, Fop y Cap, a cada tiempo de irradiación. Parte inferior: Áreas de compuesto X (área $310 \mathrm{~nm}$ ) y dímeros (área $270 \mathrm{~nm}$ ) en función del tiempo de irradiación.

Para comprobar si la fotosensibilización de $\mathrm{H}_{2} \mathrm{Nep}$ es posible con otros derivados pterínicos, como sucede con $\mathrm{H}_{2} \mathrm{Bip}$, se realizaron experimentos adicionales. En este caso, se utilizó Mep como fotosensibilizador. En la Figura 10.9 se muestran los resultados de irradiar una mezcla en aire de $\mathrm{H}_{2} \mathrm{Nep}(50 \mu \mathrm{M})$ y Mep $(114 \mu \mathrm{M})$. Como se puede apreciar, Mep no se consume, mientras que $\mathrm{H}_{2} \mathrm{Nep}$ se consume significativamente, generando principalmente el compuesto X. Además se observa una generación moderada de P2 y P3 y pequeñas cantidades de Nep y $\mathrm{H}_{2}$ Xap. 

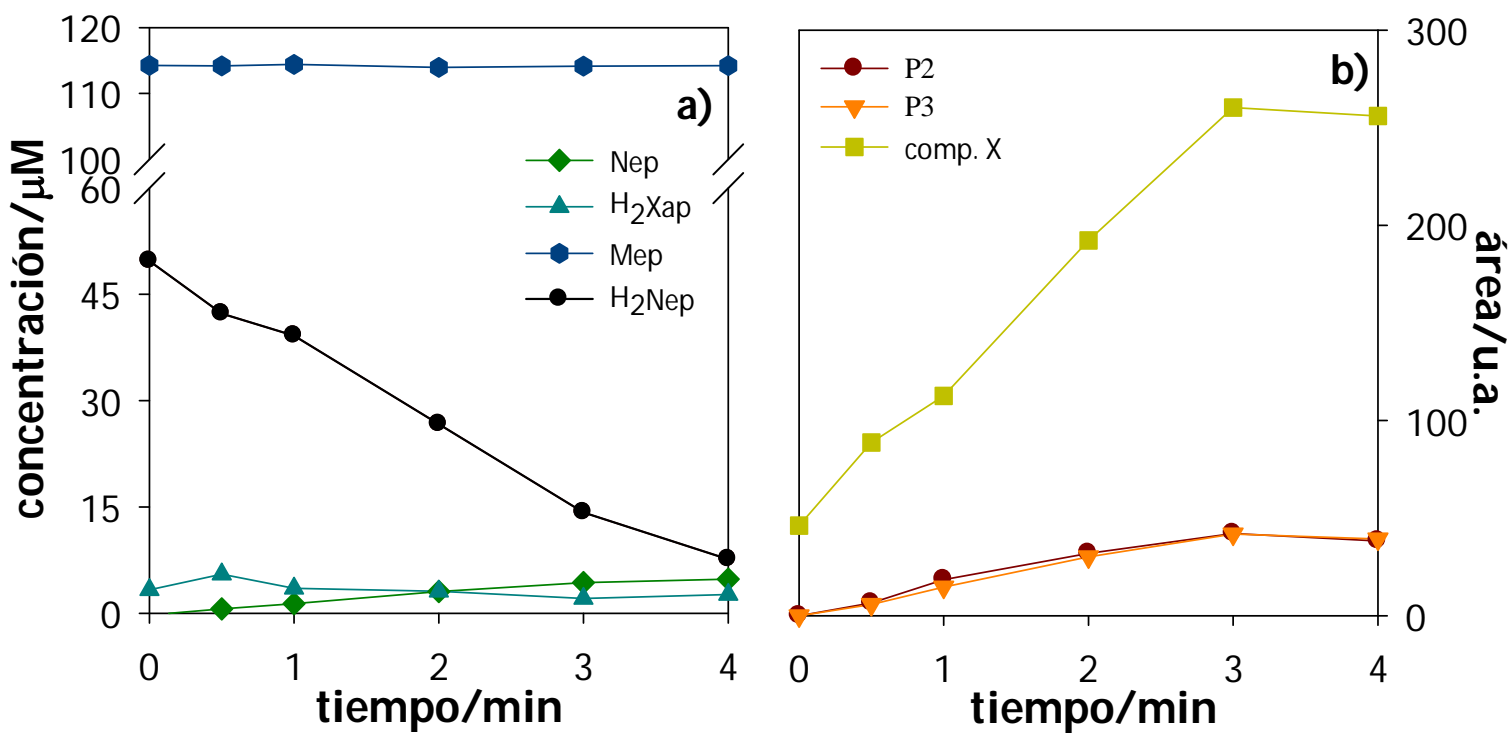

Figura 10.9 Perfiles de concentración de reactivos y productos en función del tiempo de irradiación. Sistema de irradiación I y Equipo HPLC I. a) $\left[\mathrm{H}_{2} \mathrm{Nep}_{0}=50 \mu \mathrm{M}\right.$, $[\mathrm{Mep}]_{0}=114$ $\mu \mathrm{M}, \mathrm{pH}=7,1$, b) Áreas de compuesto X (área $310 \mathrm{~nm}$ ) y P2 y P3 (área $270 \mathrm{~nm}$ ) en función del tiempo de irradiación.

Asimismo, se realizaron experimentos con el Sistema de irradiación II, excitando la solución mezcla a $380 \mathrm{~nm}$. Como se explicó anteriormente, en estas condiciones de irradiación sólo la pterina oxidada (Nep o Mep) es capaz de absorber la radiación. Como se observa en la Figura 10.10, el comportamiento es el mismo al descripto en los párrafos anteriores. Es decir, existe una fotosensibilización de $\mathrm{H}_{2} \mathrm{Nep}$ a partir de una pterina oxidada que genera como producto principal el compuesto X.
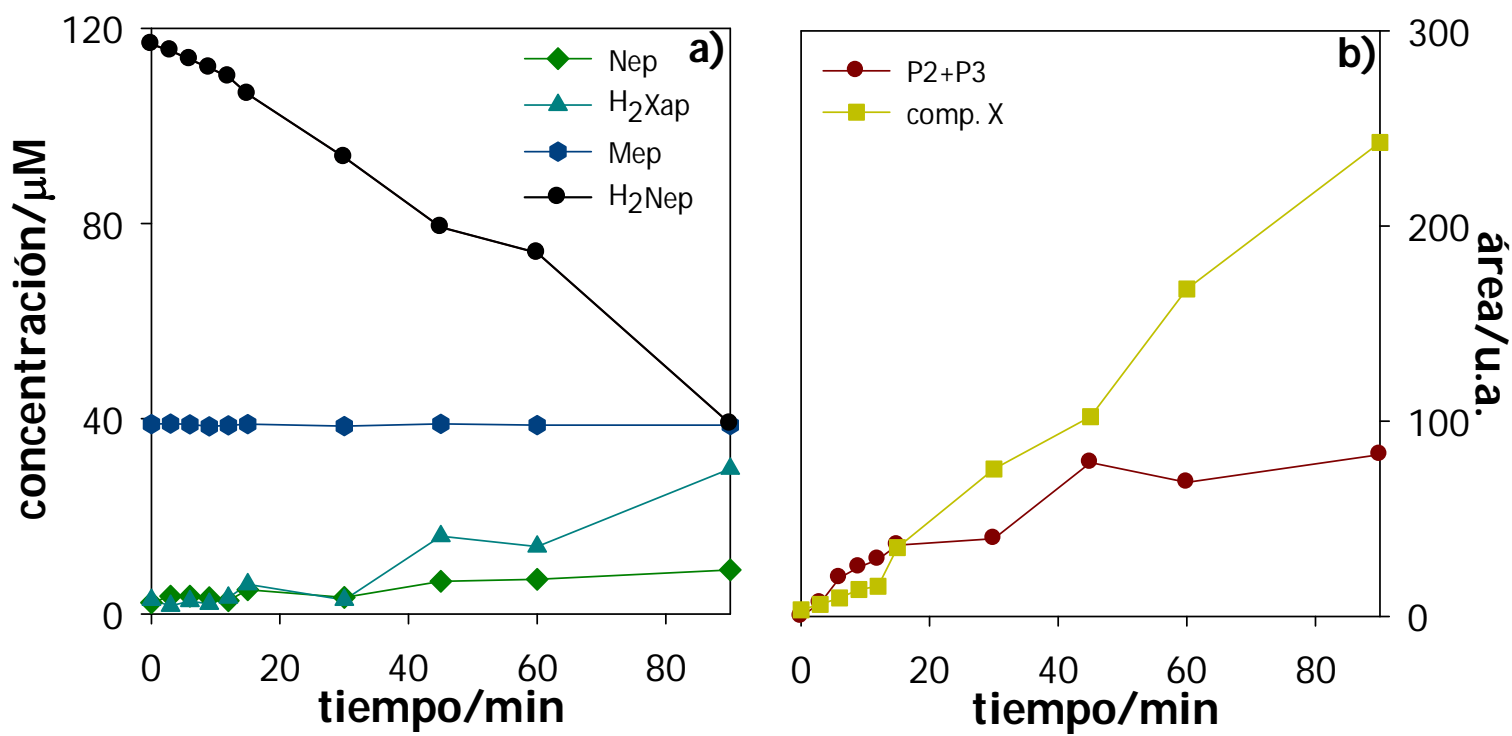

Figura 10.10 Perfiles de concentración de reactivos y productos en función del tiempo de irradiación. Sistema de irradiación II y Equipo HPLC II. a) $\left[\mathrm{H}_{2} \mathrm{Nep}_{0}=117 \mu \mathrm{M},[\mathrm{Mep}]_{0}=39\right.$ $\mu \mathrm{M}, \mathrm{pH}=6,9$, b) Áreas de compuesto X (área $310 \mathrm{~nm}$ ) y P2 y P3 (área $270 \mathrm{~nm}$ ) en función del tiempo de irradiación.

Por lo tanto, queda claro que $\mathrm{H}_{2} \mathrm{Nep}$ sufre un proceso de fotosensibilización por parte de Nep y otras pterinas oxidadas. Pero a diferencia de $\mathrm{H}_{2} \mathrm{Bip}$, el producto 
mayoritario de este proceso no es su análogo oxidado (Nep) sino el compuesto X. Este compuesto desconocido no puede actuar como fotosensibilizador en estos casos, ya que casi no absorbe radiación a 350 o 380 nm (ver espectro en Capítulo 8, Figura 8.3). Por otro lado, si bien se observa una pequeña generación de pterinas oxidadas ( $10 \%)$, esta cantidad no es suficiente como para producir una aceleración en el consumo de $\mathrm{H}_{2} \mathrm{Nep}$, motivo por el cual no se observa el efecto auto-fotocatalítico que sí se aprecia para $\mathrm{H}_{2}$ Bip.

\subsection{Conclusiones}

En este capítulo se demostró que ambos dihidroderivados, $\mathrm{H}_{2} \mathrm{Bip}$ y $\mathrm{H}_{2} \mathrm{Nep}$, sufren una oxidación fotosensibilizada por pterinas oxidadas. En el caso de $\mathrm{H}_{2} \mathrm{Bip}$, el producto principal de la fotosensibilización es otra pterina oxidada (Bip), lo cual explica el comportamiento cinético auto-fotocatalítico explicado previamente en el Capítulo 9. Para el caso de $\mathrm{H}_{2} \mathrm{Nep}$, el producto de fotosensibilización es principalmente un compuesto que no absorbe radiación a la longitud de onda de excitación (compuesto X), y por lo tanto, este proceso no participa en la fotoquímica directa de $\mathrm{H}_{2} \mathrm{Nep}$, como se detalló en el Capítulo 8.

El mecanismo de fotosensibilización propuesto se aprecia en el Esquema 10.1.

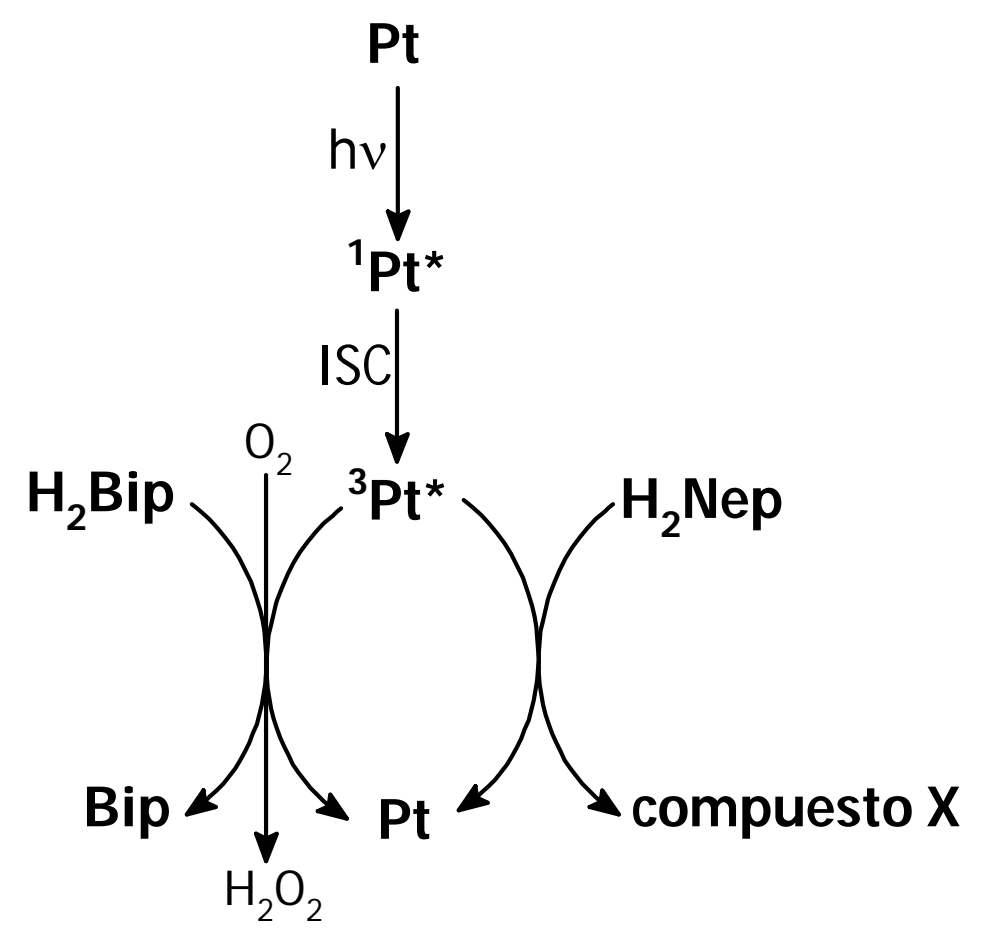

Esquema 10.1 Mecanismo de fotosensibilización para $\mathrm{H}_{2} \mathrm{Bip}$ y $\mathrm{H}_{2} \mathrm{Nep}$. 


\subsection{Referencias}

1. J. Widengren, Ü. Mets and R. Rigler, Fluorescence correlation spectroscopy of triplet states in solution: A theoretical and experimental study, Journal of Physical Chemistry, 1995, 99, 13368-13379.

2. R. D. Vierstra, K. L. Poff, E. B. Walker and P. S. Song, Effect of Xenon on the Excited States of Phototropic Receptor Flavin in Corn Seedlings, Plant Physiology, 1981, 67, 996-998.

3. P. A. W. Van den Berg, J. Widengren, M. A. Hink, R. Rigler and A. J. W. G. Visser, Fluorescence correlation spectroscopy of flavins and flavoenzymes: Photochemical and photophysical aspects, Spectrochimica Acta - Part A: Molecular and Biomolecular Spectroscopy, 2001, 57, 2135-2144.

4. M. S. Kritsky, T. A. Lyudnikova, E. A. Mironov and I. V. Moskaleva, The UV radiation-driven reduction of pterins in aqueous solution, J. Photochem. Photobiol. B, 1997, 39, 43-48.

5. R. T. Parker, Room temperature phosphorescence of selected pteridines, Analytical Chemistry, 1979, 51, 1921-1926.

6. F. M. Cabrerizo, M. L. Dántola, A. H. Thomas, C. Lorente, A. M. Braun, E. Oliveros and A. L. Capparelli, Photooxidation of pterin in aqueous solutions: biological and biomedical implications, Chemistry \& biodiversity, 2004, 1, 1800-1811.

7. F. M. Cabrerizo, C. Lorente, M. Vignoni, R. Cabrerizo, A. H. Thomas and A. L. Capparelli, Photochemical behavior of 6-methylpterin in aqueous solutions: Generation of reactive oxygen species, Photochemistry and Photobiology, 2005, 81, 793-801.

8. A. H. Thomas, C. Lorente, A. L. Capparelli, C. G. Martínez, A. M. Braun and E. Oliveros, Singlet oxygen $\left({ }^{1} \Delta_{\mathrm{g}}\right)$ production by pterin derivatives in aqueous solutions, Photochemical and Photobiological Sciences, 2003, 2, 245-250.

9. M. L. Dántola, A. H. Thomas, A. M. Braun, E. Oliveros and C. Lorente, Singlet oxygen $\left(\mathrm{O}_{2}\left({ }^{1} \Delta_{\mathrm{g}}\right)\right)$ quenching by dihydropterins, Journal of Physical Chemistry A, 2007, 111, 4280-4288.

10. A. A. Krasnovskii Jr, Photosensitized luminescence of singlet oxygen in aqueous solutions, Biophysics, 1979, 24, 769-771.

11. G. Petroselli, R. Erra-Balsells, F. M. Cabrerizo, C. Lorente, A. L. Capparelli, A. M. Braun, E. Oliveros and A. H. Thomas, Photosensitization of 2'-deoxyadenosine-5'-monophosphate by pterin, Organic and Biomolecular Chemistry, 2007, 5, 2792-2799.

12. G. Petroselli, M. L. Dántola, F. M. Cabrerizo, A. L. Capparelli, C. Lorente, E. Oliveros and A. H. Thomas, Oxidation of 2'-deoxyguanosine 5'-monophosphate photoinduced by pterin: Type I versus type II mechanism, Journal of the American Chemical Society, 2008, 130, 3001-3011.

13. A. H. Thomas, G. Suárez, F. M. Cabrerizo, R. Martino and A. L. Capparelli, Study of the photolysis of folic acid and 6-formylpterin in acid aqueous solutions, Journal of Photochemistry and Photobiology A: Chemistry, 2000, 135, 147-154. 


\section{Capítulo 11}

\section{Caracterización y estabilidad térmica de los fotodímeros}

Tal como se explicó en los Capítulos 8 y 9, 7,8-dihidrobiopterina ( $\left.\mathrm{H}_{2} \mathrm{Bip}\right)$ y 7,8dihidroneopterina $\left(\mathrm{H}_{2} \mathrm{Nep}\right)$ generan, bajo irradiación UV-A, dímeros que poseen pesos moleculares exactamente igual al doble del peso de cada reactivo. Sin embargo, nada se ha dicho sobre la estructura de estos compuestos hasta el momento. Por otro lado, algunos controles realizados con las soluciones irradiadas mostraron que los espectros de absorción de dichas soluciones sufren cambios una vez interrumpida la irradiación. Esto indica que se producen reacciones térmicas que involucrarían a los dímeros formados.

A la luz de estas consideraciones, este capítulo se centra en el estudio de la estructura y estabilidad de los dímeros. Se realizaron análisis de ${ }^{1} \mathrm{H}$-RMN para obtener información estructural, mientras que la estabilidad de dichos compuestos se evaluó a través de un estudio cinético de soluciones de $\mathrm{H}_{2} \mathrm{Bip}$ y $\mathrm{H}_{2} \mathrm{Nep}$, previamente irradiadas.

\subsection{Estabilidad térmica de los dímeros}

Para estudiar la estabilidad térmica de los dímeros formados, se irradiaron soluciones de ambos dihidroderivados a pH $\sim 7$, con el Sistema de irradiación I (Sección 4.3). Luego estas soluciones se mantuvieron en oscuridad y se registraron los espectros de absorción a distintos intervalos de tiempo (tiempo de oscuridad, to). Los resultados observados para ambos compuestos fueron muy similares. Como se aprecia en la Figura 11.1, la banda de menor energía característica de las dihidropterinas aumenta mientras que disminuye la intensidad a $\sim 250 \mathrm{~nm}$. Esto demuestra que los dímeros son inestables térmicamente.

Para evaluar la participación del $\mathrm{O}_{2}$ en este proceso, algunas de las soluciones irradiadas en ausencia de dicho gas se mantuvieron en condiciones anaeróbicas, mientras que otras fueron aireadas inmediatamente después de interrumpir la irradiación. Los resultados en ambas condiciones fueron muy similares para ambos 
compuestos. Los mismos sugieren que el $\mathrm{O}_{2}$ no participa en la reacción en oscuridad que da lugar a la desaparición o consumo neto de los dímeros.
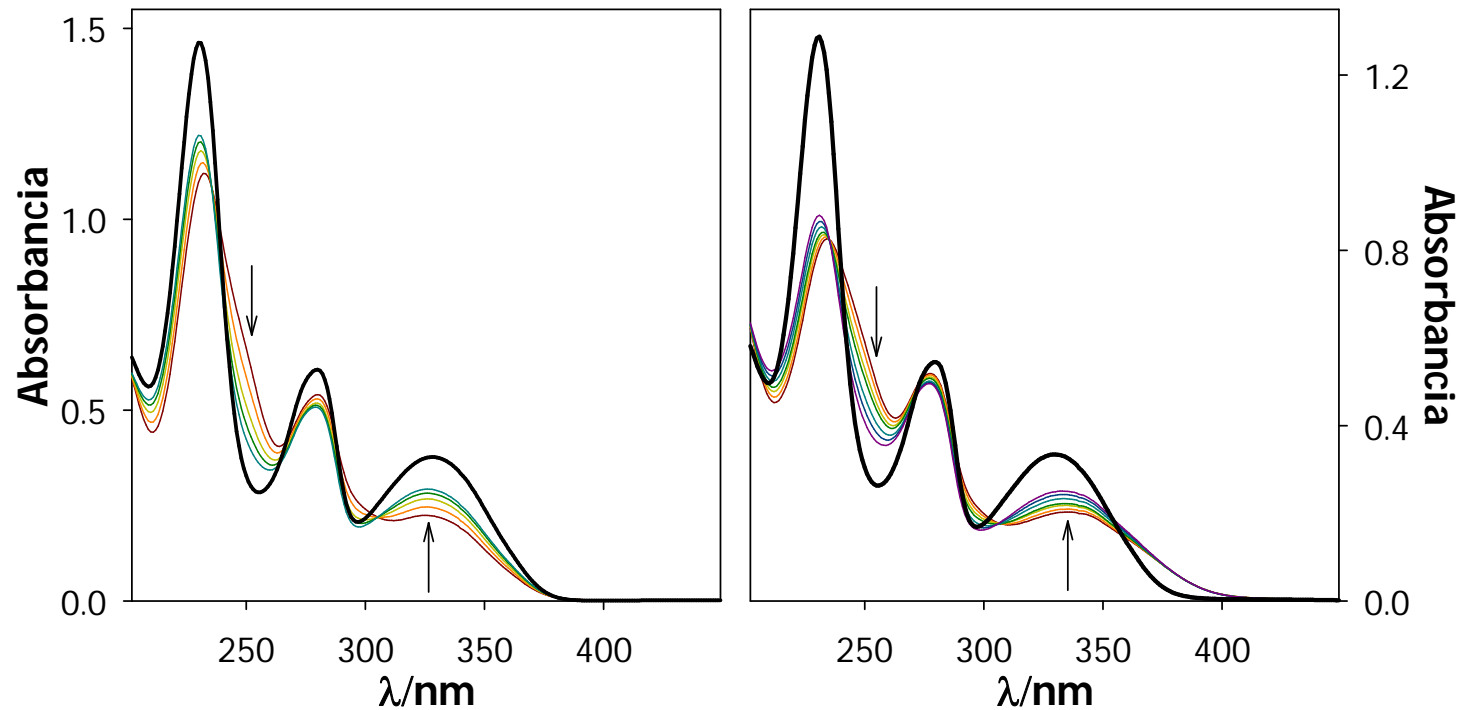

Figura 11.1 Evolución de los espectros de absorción de las soluciones de los dihidroderivados en función del tiempo, después de interrumpida la irradiación realizada en presencia de $\mathrm{O}_{2}(\mathrm{ti}=6 \mathrm{~min})$. a) $\left[\mathrm{H}_{2} \mathrm{Nep}\right]_{0}=150 \mu \mathrm{M}, \mathrm{pH}=7,0$, to total $=5 \mathrm{~h} 15$ $\min$; b) $\left[\mathrm{H}_{2} \mathrm{Bip}_{0}=144 \mu \mathrm{M}, \mathrm{pH}=7,1\right.$ to total $=6 \mathrm{~h} 40 \mathrm{~min}$. Con fines comparativos se presenta el espectro correspondiente a cada solución previa irradiación (línea gruesa negra). Sistema de irradiación I y camino óptico $=0,4 \mathrm{~cm}$.

En otro grupo de experimentos se procedió en forma similar a lo expuesto en los párrafos precedentes aunque las soluciones fueron analizadas por HPLC a distintos tiempos luego de interrumpir la irradiación. En la Figura 11.2, se muestran los cromatogramas de una solución de $\mathrm{H}_{2} \mathrm{Nep}$ irradiada por 6 min y conservada en oscuridad por más de $7 \mathrm{~h}$. Como se puede apreciar, luego de interrumpida la irradiación las áreas de los picos de los dímeros P2 y P3 ( $\operatorname{tr}=3,5$ y 3,9, respectivamente) disminuyen con el tiempo de oscuridad, mientras que aumentan las áreas correspondientes a los picos de $\mathrm{P} 1$ y de $\mathrm{H}_{2} \mathrm{Nep}$ ( $\operatorname{tr}=3,0$ y 6,0, respectivamente). Para el caso de $\mathrm{H}_{2}$ Bip se observó exactamente el mismo comportamiento. 


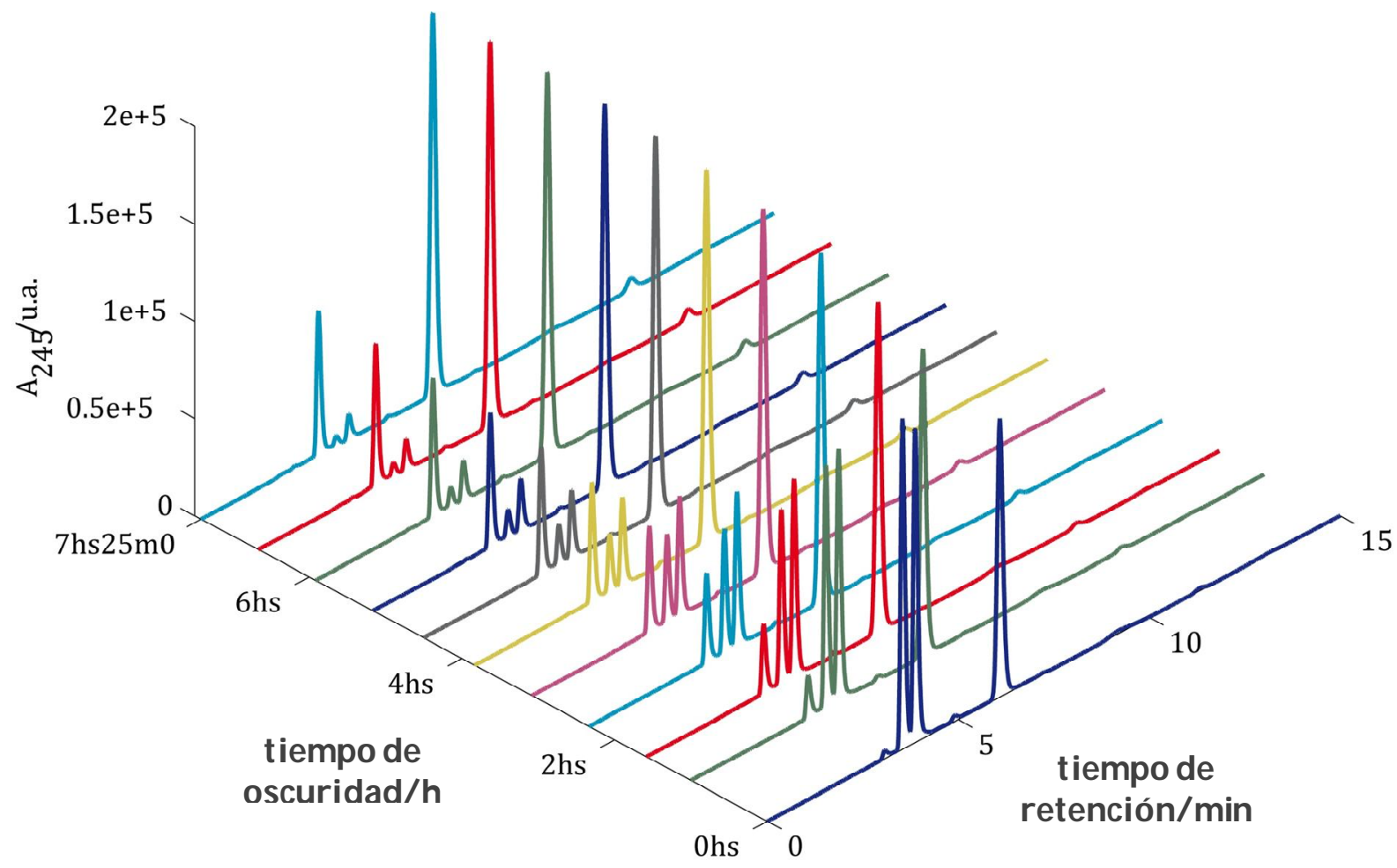

Figura 11.2 Cromatogramas $\left(\lambda_{\mathrm{an}}=245 \mathrm{~nm}\right)$ obtenidos en la reacción en oscuridad luego de irradiar una solución de $\mathrm{H}_{2} \mathrm{Nep}$ en presencia de $\mathrm{O}_{2}\left(\left[\mathrm{H}_{2} \mathrm{Nep}\right]_{0}=151 \mu \mathrm{M}, \mathrm{pH}=6,9\right.$, ti $=6$ min). Sistema de irradiación I y Equipo HPLC I.

En la Figura 11.3 se muestran los perfiles de concentración para los reactivos y las áreas a $270 \mathrm{~nm}$ para los dímeros P2 y P3 y a $250 \mathrm{~nm}$ para P1, en función del tiempo de oscuridad luego de interrumpida la irradiación. Como se puede apreciar, las áreas correspondientes a los dímeros disminuyen de manera proporcional en función del tiempo de oscuridad. Además, se observa que aumenta el área del producto P1, descripto anteriormente (Capítulos 8 y 9), indicando que no es un fotoproducto primario y minoritario de $\mathrm{H}_{2} \mathrm{Bip}$ y $\mathrm{H}_{2} \mathrm{Nep}$, tal como se creía inicialmente, sino un producto de la descomposición térmica de los dímeros. Por otro lado, se observa la clara recuperación del reactivo inicial, lo cual concuerda con el aumento de la intensidad de absorbancia $\mathrm{a} \approx 330 \mathrm{~nm}$. 

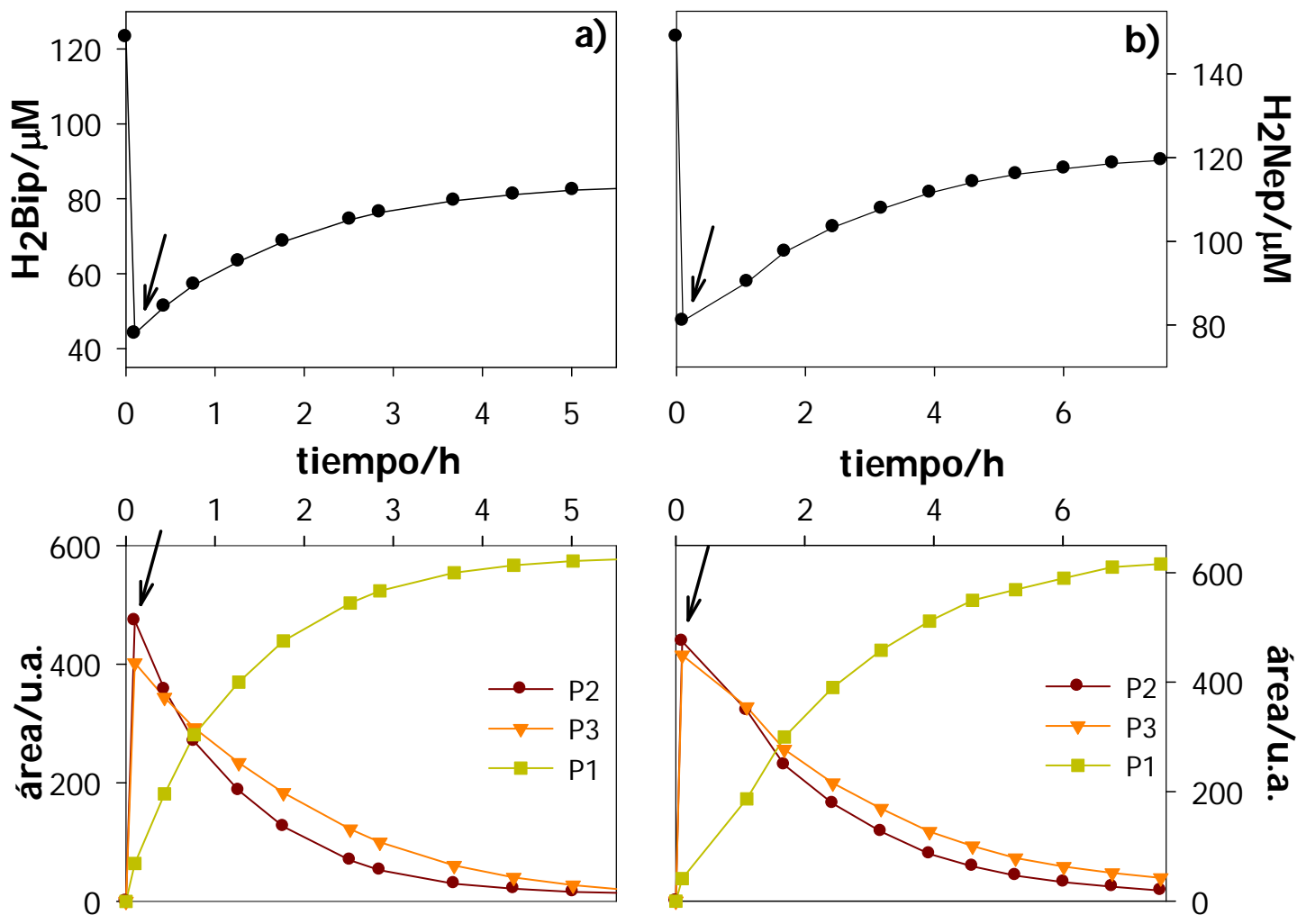

Figura 11.3 Perfiles de concentración en función del tiempo de oscuridad para los reactivos, y áreas a $270 \mathrm{~nm}$, para los dímeros P2 y P3, y a $250 \mathrm{~nm}$, para el producto P1. El tiempo 0 corresponde a la muestra sin irradiar. La flecha indica el momento en que se interrumpe la irradiación. a) $\mathrm{H}_{2} \mathrm{Bip}$ irradiado en ausencia de $\mathrm{O}_{2}\left(\left[\mathrm{H}_{2} \mathrm{Bip}_{0}=123 \mu \mathrm{M}, \mathrm{pH}=\right.\right.$ $6,2, \mathrm{ti}=10 \mathrm{~min}), \mathrm{b}) \mathrm{H}_{2} \mathrm{Nep}$. $\left(\left[\mathrm{H}_{2} \mathrm{Nep}\right]_{0}=151 \mu \mathrm{M}, \mathrm{pH}=6,9, \mathrm{ti}=6 \mathrm{~min}\right)$. Sistema de irradiación Iy Equipo HPLC I.

Comparando el reactivo consumido en la reacción fotoquímica $\left(\Delta[R]_{R \rightarrow D}\right)$ y la cantidad de reactivo regenerado en la reacción en oscuridad $\left(\Delta[R]_{D \rightarrow R}\right)$, se obtiene una relación $\left(\Delta[\mathrm{R}]_{\mathrm{R} \rightarrow \mathrm{D}}\right) /\left(\Delta[\mathrm{R}]_{\mathrm{D} \rightarrow \mathrm{R}}\right)$ de $\approx 0,5$ en todos los casos. Es decir que los dímeros en una reacción en oscuridad regeneran $\approx 50 \%$ del reactivo y el otro $\approx 50 \%$ seguramente corresponde al producto al que se ha denominado P1. Por lo tanto, si en la descomposición de los dímeros se recupera el reactivo inicial, P1 debe ser el otro monómero con alguna modificación química.

En estas condiciones experimentales, la desaparición de los dímeros y la formación de P1 y reactivo siguen una cinética de pseudo-primer orden. Por lo tanto, se determinaron las constantes de velocidad $(\mathrm{k})$ de degradación térmica de ambos dímeros. Las mismas fueron calculadas a partir de la ecuación de decaimiento de primer orden:

$$
\ln \left([\mathrm{D}] /[\mathrm{D}]_{0}\right)=-\mathrm{kt}
$$


donde $[\mathrm{D}]_{0}$ es la concentración de un dímero inmediatamente después de interrumpir la irradiación y [D] es la concentración del dímero a un determinado tiempo de reacción en oscuridad. Al no ser posible determinar las concentraciones, se realizaron los cálculos teniendo en cuenta el área de los picos de los dímeros en los cromatogramas $\left([\mathrm{D}] /[\mathrm{D}]_{0}=\right.$ $\left.[\mathrm{A}] /[\mathrm{A}]_{0}\right)$. Los valores de $\mathrm{k}$ se calcularon para ambos dímeros de los dos dihidroderivados a dos temperaturas diferentes, $21^{\circ} \mathrm{C}$ y $37^{\circ} \mathrm{C}$. En la Tabla 11.1 se puede apreciar que, para una dihidropterina en particular, P2 tiene una velocidad específica de degradación ligeramente mayor que P3. Por otra parte, al comparar los valores de k correspondientes a la degradación de los dímeros P2 y P3 obtenidos tanto a partir de $\mathrm{H}_{2}$ Bip como de $\mathrm{H}_{2} \mathrm{Nep}$, se observa que son iguales, dentro del error experimental. Este hecho sugiere o da cuenta de una similitud estructural en cuanto a la naturaleza química de cada dímero formado.

\begin{tabular}{lccc}
\hline & & \multicolumn{2}{c}{$\mathbf{k}\left(\mathbf{1 0} \mathbf{- 2}^{-2} / \mathbf{s}^{-\mathbf{1}}\right.$} \\
& & $\mathbf{2 1}{ }^{\circ} \mathbf{C}$ & $37^{\circ} \mathbf{C}$ \\
\hline $\mathbf{H}_{2} \mathbf{N e p}$ & P2 & $0,69 \pm 0,02$ & $2,7 \pm 0,3$ \\
& P3 & $0,43 \pm 0,02$ & $2,0 \pm 0,2$ \\
$\mathbf{H}_{2}$ Bip & P2 & $0,69 \pm 0,02$ & $3,1 \pm 0,2$ \\
& P3 & $0,36 \pm 0,02$ & $2,0 \pm 0,1$
\end{tabular}

Tabla 11.1 Constantes de velocidad de degradación de los dímeros P2 y P3.

\subsection{Caracterización de P1 por espectrometría de masa}

Para obtener mayor información estructural acerca de P1, se realizaron experimentos de espectrometría de masa con el Equipo MS I (Sección 5.4). Para ello, se irradiaron soluciones del dihidroderivado correspondiente durante un tiempo tal que permitió obtener una cantidad importante de dímeros. Luego de interrumpida la irradiación se mantuvieron las soluciones en oscuridad el tiempo necesario para lograr que la reacción llegue a su fin. Experimentalmente, esto se logró mediante el seguimiento de la reacción en oscuridad por espectroscopía UV-visible hasta no detectar mayores cambios en los espectros de absorción, evidenciando así la descomposición total de los dímeros (o fin de la reacción en oscuridad). Finalmente, estas muestras se analizaron por HPLC-MS. En la Figura 11.4 se puede observar el espectro de masa en modo positivo $\left(\mathrm{ESI}^{+}\right)$del pico del cromatograma correspondiente a P1, para una solución de $\mathrm{H}_{2}$ Bip irradiada 30 min con el Sistema de irradiación II, y conservada en oscuridad durante $\approx 6 \mathrm{~h}$. El ión molecular que aparece a $\mathrm{m} / \mathrm{z}=258,3$ correspondería a 
una molécula de monómero de reactivo que incorporó una molécula de $\mathrm{H}_{2} \mathrm{O}$ $\left[\mathrm{H}_{2} \mathrm{Bip}+\mathrm{H}_{2} \mathrm{O}+\mathrm{H}\right]^{+}=239+18+1$. También se observan dos picos a $\mathrm{m} / \mathrm{z}=240,3$ y 280,3 correspondientes a $\left[\mathrm{M}-\mathrm{H}_{2} \mathrm{O}+\mathrm{H}\right]+{ }^{+} \mathrm{y}$ al aducto $[\mathrm{M}+\mathrm{Na}]^{+}$, respectivamente.

Estos resultados sugieren que, cuando los dímeros se descomponen, un monómero regenera el reactivo inicial mientras que el otro incorporaría una molécula de $\mathrm{H}_{2} \mathrm{O}$. Esto coincide también con los espectros de absorción de P1, mostrados en los capítulos anteriores (Figuras 8.2 y 9.2), ya que al incorporar una molécula de $\mathrm{H}_{2} \mathrm{O}$ se perdería la conjugación del anillo pirazínico y, consecuentemente, la banda de absorción centrada alrededor de $330 \mathrm{~nm}$.

Es importante aclarar que la obtención de los espectros de masa de P1 fue muy dificultosa. Esto puede deberse, en parte, a que P1 tendría una elevada polaridad y, en las condiciones en las cuales se realizan las corridas cromatográficas, tiene un tiempo de retención extremadamente corto, saliendo casi junto con el frente de solvente. Esto hace que sea muy dificultosa la obtención de espectros de masa sin contaminaciones. Otros motivos podrían ser de carácter químico, dado que P1 podría reaccionar y descomponerse, ya que se desconoce su estabilidad térmica. Para el caso de $\mathrm{H}_{2} \mathrm{Bip}$ también pudo detectarse P1 en modo negativo (ESI-), con la presencia de un ión molecular en $\mathrm{m} / \mathrm{z}=256$ correspondiente a P1 ([M-H]-). Por el contrario, en el caso de $\mathrm{H}_{2} \mathrm{Nep}$, no fue posible en ninguno de los dos modos de análisis (ESI+ ${ }^{+}$y ESI-) obtener espectros de masa adecuados.

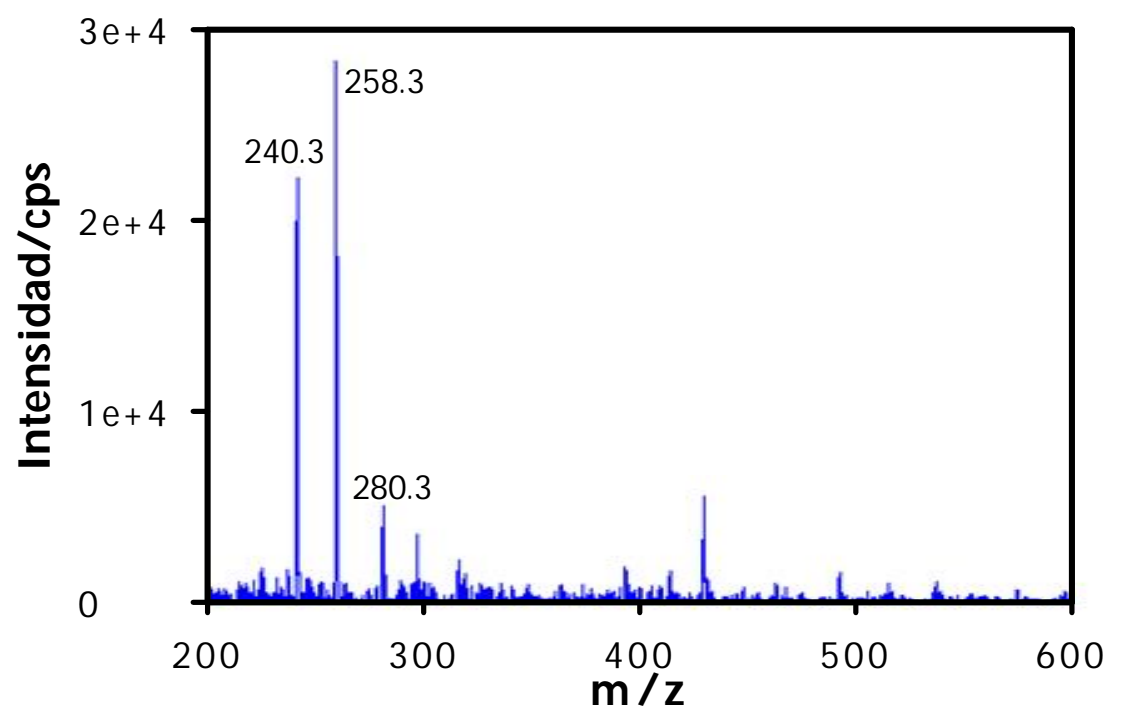

Figura 11.4 Espectro de masa (modo ESI+) del pico correspondiente a P1 de una solución de $\mathrm{H}_{2}$ Bip irradiada en condiciones anaeróbicas ( $\mathrm{ti}=30 \mathrm{~min}$ ) y conservada en oscuridad por $\sim 6$ h. Sistema de irradiación II y Equipo MS I. 


\subsection{Análisis de la estructura de los dímeros}

Para obtener mayor información respecto de la estructura molecular de los dímeros formados, se recurrió al uso de ${ }^{1} \mathrm{H}-\mathrm{RMN}$ para el análisis de las soluciones irradiadas. Se utilizó $\mathrm{D}_{2} \mathrm{O}$ como solvente, donde sólo se detectan protones no intercambiables, como ya se detalló en la Sección 5.5. La concentración de las soluciones de dihidropterinas utilizadas variaron en un rango comprendido entre 1,4-1,8 mM, y el pD siempre se estableció alrededor de 7.

Primero, se registraron los espectros de ${ }^{1} \mathrm{H}-\mathrm{RMN}$ de las soluciones patrón de $\mathrm{H}_{2}$ Bip y $\mathrm{H}_{2} \mathrm{Nep}$ (Figura 11.5). Para $\mathrm{H}_{2}$ Bip se detectaron 4 señales en los siguientes valores de $\delta(\mathrm{ppm}): \delta=4,18\left(\mathrm{dd}, \mathrm{J}_{\mathrm{gem}}=0,5 \mathrm{~Hz}\right), 4,09\left(\mathrm{~d}, \mathrm{~J}_{\alpha \beta}=6 \mathrm{~Hz}\right), 3,99(\mathrm{~m})$ y 1,21 (d, J $\mathrm{J}_{\beta \gamma}$ $=6,5 \mathrm{~Hz}$ ). Las intensidades relativas de estas señales medidas a partir de las integrales de cada pico fueron 2:1:1:3. Estas señales corresponden a los dos protones unidos al C7 del anillo, al $\mathrm{C} \alpha$, al $\mathrm{C} \beta$ y a los tres protones unidos al $\mathrm{C} \gamma$ de la cadena alifática, respectivamente. En el caso de $\mathrm{H}_{2} \mathrm{Nep}$ se detectaron 4 señales con intensidades relativas de 3:1:1:1. La primera señal que integra para tres protones, corresponde a dos señales superpuestas: dos dobletes con baja resolución correspondientes a los protones del C7 $(\delta=4,21)$ y un doblete del $\mathrm{H}_{\alpha}$ a $\delta=4,20\left(\mathrm{~d}, \mathrm{~J}_{\alpha \beta}=6 \mathrm{~Hz}\right)$. Las otras señales tienen valores de $\delta$ de $3,88(\mathrm{~m}), 3,78\left(\mathrm{dd}, \mathrm{J}_{\beta \gamma \mathrm{a}}=3,5 \mathrm{~Hz}\right.$ y $\mathrm{J}_{\gamma \mathrm{\gamma} \mathrm{b}}=12 \mathrm{~Hz}$ ) y $3,65\left(\mathrm{dd}, \mathrm{J}_{\beta \gamma \mathrm{b}}=6,5 \mathrm{~Hz}\right.$ y $\mathrm{J}_{\gamma \mathrm{\gamma} \mathrm{b}}=12$ $\mathrm{Hz}$ ) que corresponden al protón $\mathrm{H}_{\beta}$ y a los protones metilénicos $\mathrm{H} \gamma_{\mathrm{a}}$ and $\mathrm{H} \gamma_{\mathrm{b}}$, respectivamente. ${ }^{1}$

En el caso de las soluciones de $\mathrm{H}_{2} \mathrm{Bip}$, previamente a su irradiación, las mismas fueron burbujeadas con Ar, evitando así que tenga lugar la reacción correspondiente a la vía oxidativa (ver Esquema 9.2). En el caso de las soluciones de $\mathrm{H}_{2} \mathrm{Nep}$ esto no fue necesario debido a que durante la reacción fotoquímica de este dihidroderivado solo tiene lugar la dimerización, por lo que se trabajó en atmósfera de aire. Luego se irradiaron las soluciones utilizando el Sistema de irradiación I durante $\sim 40 \mathrm{~min}$. 
a)<smiles>CC(O)C(O)C1=Nc2c(nc(N)[nH]c2=O)NC1([18OH])[18OH]</smiles>
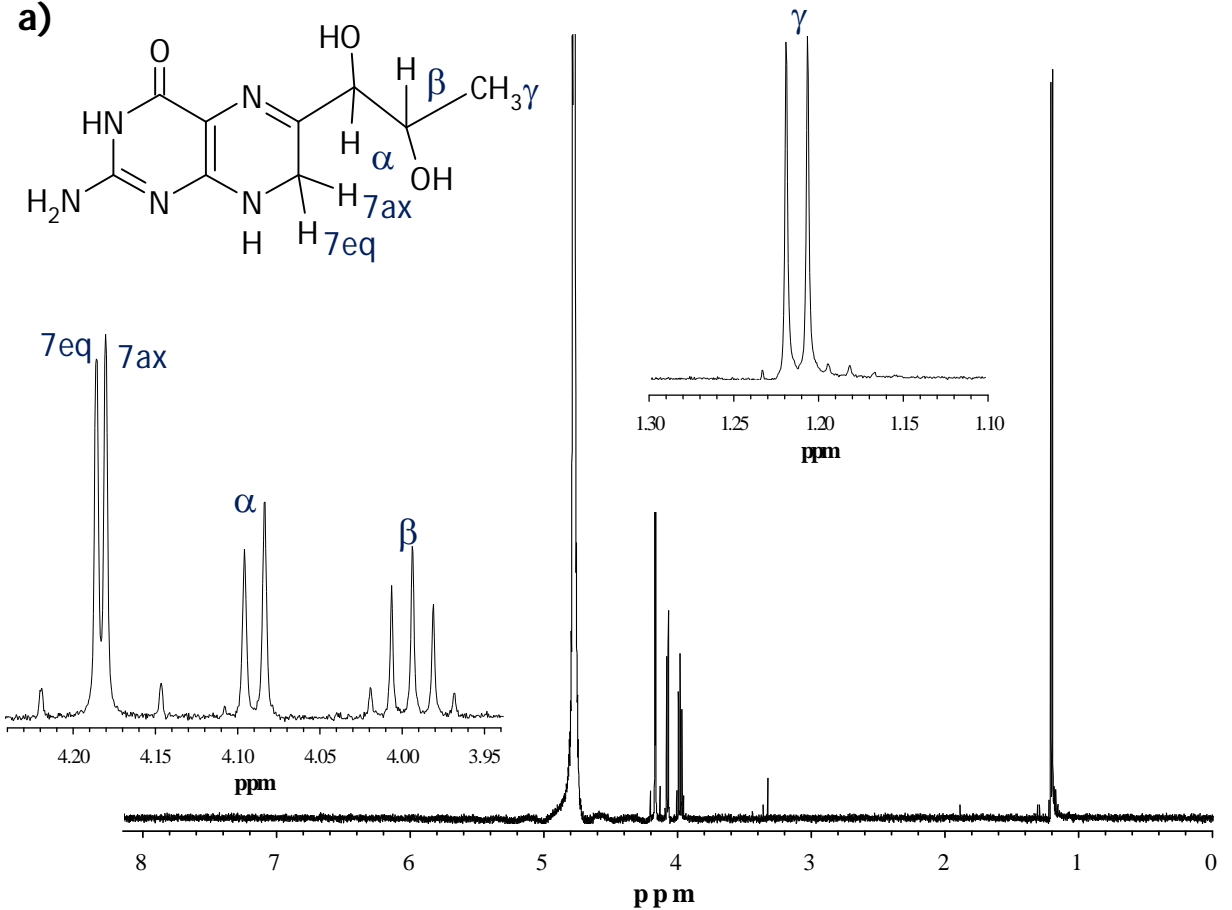

b)

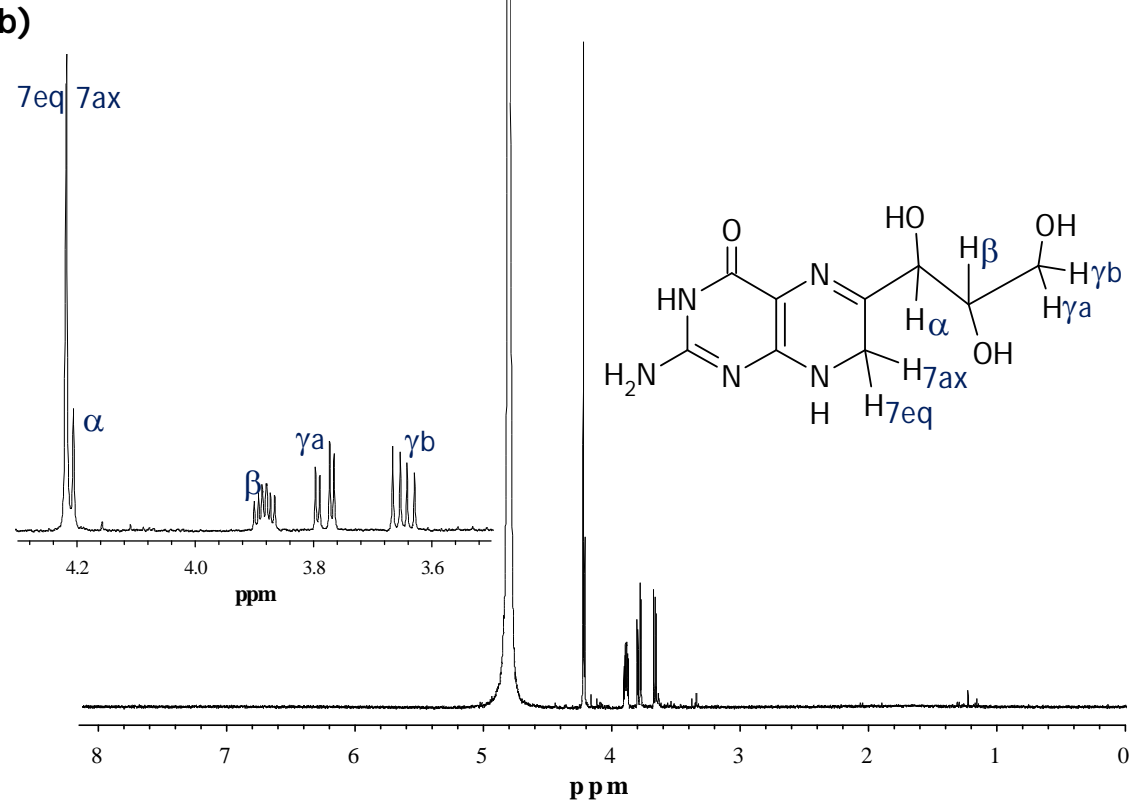

Figura 11.5 Espectro de 1H-NMR de los patrones. a) $\mathrm{H}_{2} \mathrm{Bip}$, b) $\mathrm{H}_{2} \mathrm{Nep}$.

A continuación se registraron los espectros ${ }^{1} \mathrm{H}-\mathrm{RMN}$. Un análisis preliminar muestra una disminución de las señales de los reactivos junto a la aparición de nuevas señales correspondientes a los productos (Figura 11.6.b). Sabiendo que los dímeros no son estables, estas soluciones se mantuvieron en oscuridad y se registraron los espectros a distintos tiempos. Se pudo apreciar una dependencia temporal de la intensidad de algunas señales (Figura 11.6.c). Según la evolución de las mismas en el tiempo, estas nuevas señales se clasificaron en dos grupos: aquellas cuya intensidad disminuye con el tiempo y aquellas otras que, por el contrario, aumentan. El primer 
grupo de señales se atribuyó a los dímeros, dado que, como se detalló en la sección anterior, son inestables. Por otro lado, en el segundo grupo de señales se detectaron, las correspondientes al reactivo que se regenera y las que pertenecen a los productos de la reacción en oscuridad de los dímeros, es decir, P1.

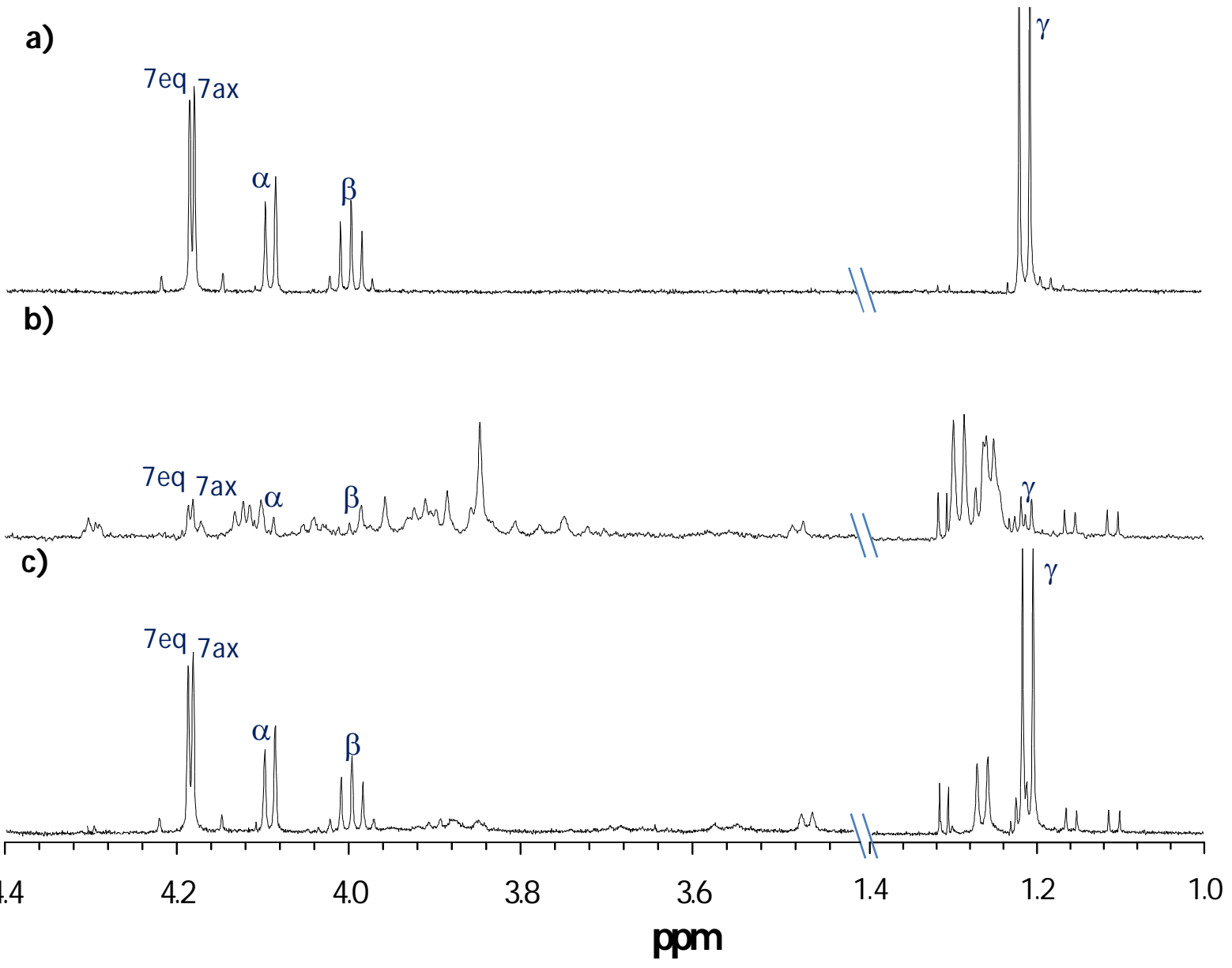

Figura 11.6 Parte de espectros de ${ }^{1} \mathrm{H}-\mathrm{RMN}$ de una solución de $\mathrm{H}_{2} \mathrm{Bip}$. a) antes de irradiar, b) inmediatamente después de irradiar, c) después de un tiempo de oscuridad.

Para formar los compuestos diméricos, dos moléculas de reactivo deben reaccionar. Teniendo en cuenta que el rendimiento cuántico de consumo de reactivo no depende con la concentración, debe ocurrir una fototautomerización 0 fotoisomerización previa a la dimerización. Es decir, debe formarse un intermediario isomérico o tautomérico que luego pueda reaccionar con otra molécula de reactivo en estado basal (Sección 8.4). Un intermediario muy reactivo y que ya se ha detectado en otros casos, por ejemplo en la fotólisis de Bip y Nep (Capítulo 6), es el 5,8dihidroderivado. De esta manera, una 5,8 dihidropterina y una 7,8-dihidropterina podrían reaccionar mediante una foto-ciclodimerización tipo [2+2] a partir de los dobles enlaces presentes en distintas posiciones de cada molécula. Por un lado, podría ocurrir la cicloadición a través de los dobles enlaces C9=C10 (Capítulo 2) de las uniones del 
anillo pirazina y pirimidina, tal como sucede en el caso de timina y uracilo. ${ }^{2,} 3$ Por lo tanto, si este tipo de reacción estuviese ocurriendo en el caso de $\mathrm{H}_{2} \mathrm{Bip}$ o $\mathrm{H}_{2} \mathrm{Nep}$, entonces, en el espectro de ${ }^{1} \mathrm{H}-\mathrm{RMN}$, debería observarse una señal correspondiente a los dímeros ubicada entre 8 a 6 ppm debida a la presencia del protón olefínico del C7 (Figura 2.3). Sin embargo, esta señal no aparece en el espectro de $\mathrm{H}_{2} \mathrm{Bip}$ irradiada, motivo por el cual puede descartarse este tipo de estructuras.

Por otro lado, la cicloadición podría tener lugar entre el doble enlace C6=C7 del anillo pirazínico del monómero 5,8-dihidroderivado con el doble enlace N5=C6 del monómero 7,8-dihidroderivado, formando un azo-ciclobutano. De esta manera, se pueden formar cuatro dímeros distintos: primero por combinaciones syn o anti de los anillos y, para cada uno de ellos, los isómeros cabeza-cabeza (head-to-head $(\mathrm{h}, \mathrm{h})$ ) 0 cabeza-cola (head-to-tail $(\mathrm{h}, \mathrm{t})$ ). Estos últimos, relativos a la posición de los grupos carbonilos del anillo pirimidina. Consecuentemente, las dos cadenas alifáticas R están en distinta posición en el azo-ciclobutano: en dos dímeros se encuentran en posición 1,3 uno con respecto al otro, generando el anti-Ranti y el syn-Rsyn, mientras que en los otros dos dímeros las cadenas alifáticas $\mathrm{R}$ quedan en $\mathrm{C}$ vecinos (posición 1,2) generando los anti-Rtrans y syn-Rcis. Los cuatro isómeros se representan en el Esquema 11.1.
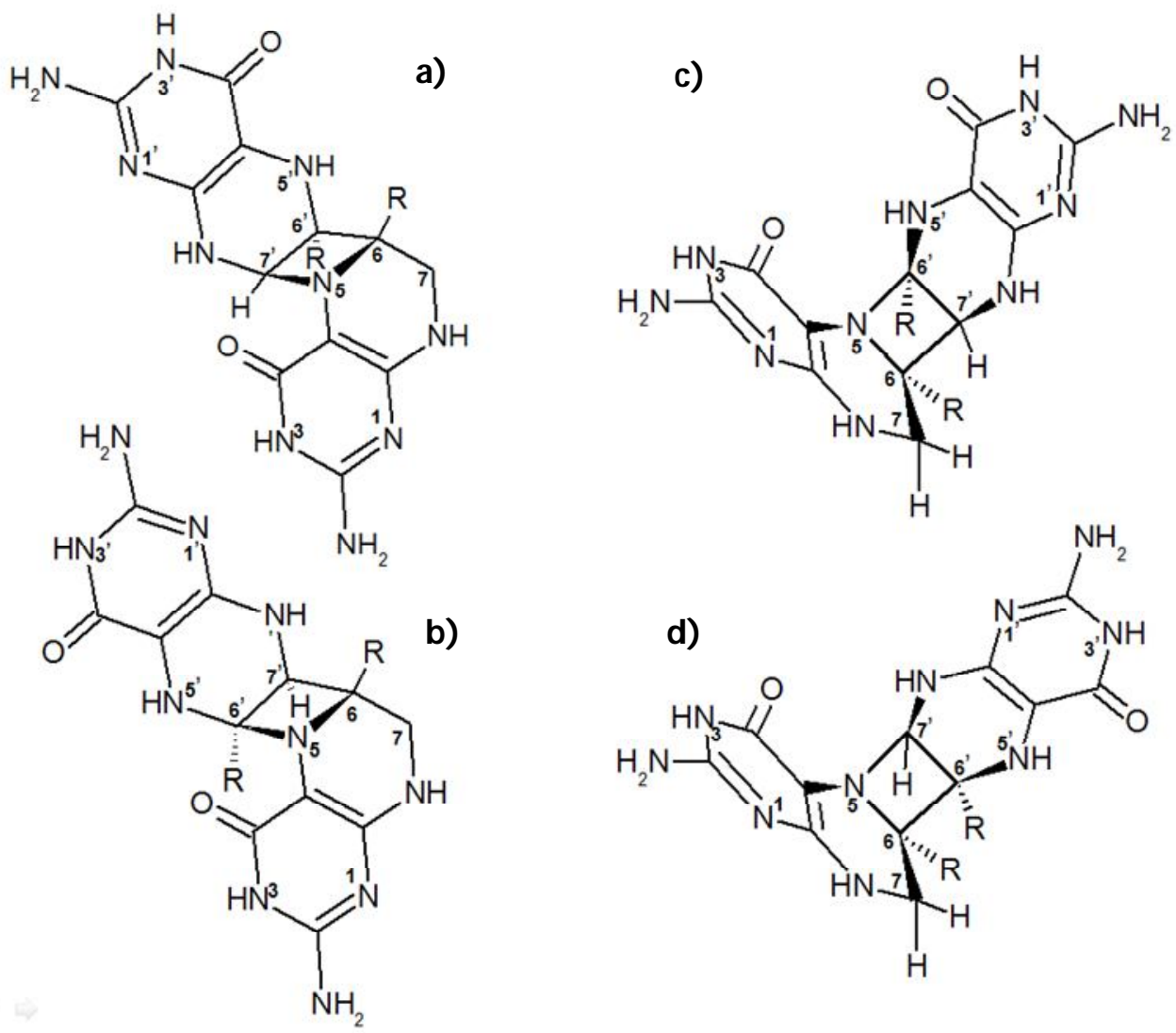

Esquema 11.1 Estructuras propuestas para los dímeros de $\mathrm{H}_{2} \mathrm{Bip}$ y $\mathrm{H}_{2} \mathrm{Nep}$. a) anti-Rtrans, b) anti-Ranti, c) syn-Rsyn, d) syn-Rcis. 
Teniendo en cuenta estas posibles estructuras de dímeros, se analizaron los espectros de ${ }^{1} \mathrm{H}-\mathrm{RMN}$ obtenidos para las soluciones irradiadas de $\mathrm{H}_{2}$ Bip y $\mathrm{H}_{2} \mathrm{Nep}$. Si bien estos espectros son muy complejos de analizar, pueden derivarse algunas conclusiones.

Tomando $\mathrm{H}_{2}$ Bip como ejemplo y analizando más detalladamente los espectros de ${ }^{1} \mathrm{H}$-RMN, en la Figura 11.6 se observa que en las soluciones irradiadas aparecen dobletes no resueltos en la zona donde aparecen los metilos (entre $\delta 1,0$ y 1,5 ). Estos picos aparecen como dos dobletes superpuestos centrados en $\delta 1,29$ y otros dos dobletes alrededor de $\delta 1,26$ y 1,25, que corresponden a los $\mathrm{H} \gamma$ y $\mathrm{Hy}$ ' de dos dímeros. Asimismo, las concentraciones de ambos dímeros evaluadas por las integrales en el espectro ${ }^{1} \mathrm{H}$ RMN son muy similares. Esto se corresponde con lo observado en el HPLC: sólo dos picos y de áreas similares (Capítulos 8 y 9). Es decir que lo más probable es que se generen solo dos de los cuatro dímeros mostrados en el Esquema 11.1.

Por otro lado, en la zona comprendida entre $\delta 3,6$ y 4,4 aparecen varios picos con multiplicidad singletes y dobletes. Según la información descripta en la literatura, ${ }^{4,5}$ estos picos corresponderían a protones metilénicos o metínicos unidos a la estructura tipo tetrahidropirazina (THP). Las estructuras tipo THP de uno de los monómeros de los dímeros propuestos en el Esquema 11.1 contiene un grupo metilénico $\left(\mathrm{H}_{2} 7\right)$ mientras que el otro monómero tiene un protón metínico en el $C\left(7^{\prime}\right)$, carbono que pertenece al azo-ciclobutano. Una señal importante en forma de singlete ancho (o doblete mal resuelto) aparece a $\delta 3,84$, la cual podría pertenecer a $\mathrm{H}_{2} 7$ de los dos dímeros formados. Además, se observaron dos singletes a $\delta 3,88$ y 3,95 que pueden atribuirse a los protones metínico ( $\mathrm{H7}^{\prime}$ ) de cada dímero. Con respecto a los protones de la cadena alifática, se podría realizar la siguiente asignación de señales: $\mathrm{H \alpha}$ y $\mathrm{H}^{\prime}$ aparecen como dobletes centrados en $\delta 3,84 ; 3,91 ; 4,11$ y 4,12, mientras que $\mathrm{H} \beta$ y $\mathrm{H} \beta^{\prime}$ aparecen como multipletes a $\delta 3,74 ; 3,80 ; 3,90$ y 4,04 .

Es muy difícil discriminar entre las posibles estructuras de los dímeros a partir de los ${ }^{1} \mathrm{H}-\mathrm{RMN}$ que, ciertamente, presentan una elevada complejidad debido a la presencia de varios fotoproductos, alguno de ellos muy inestables. Por tal motivo, es necesario tener en cuenta otros aspectos. Por ejemplo, el dímero syn-Rcis podría descartarse debido a que posee un gran impedimento estérico en su estructura. Con respecto a las otras tres estructuras, podría existir una interacción vía puentes de $\mathrm{H}$ entre los grupos $\mathrm{OH}$ de las dos cadenas alifáticas, que podría favorecer una previa orientación de las dos 
moléculas de reactivo antes de la ciclación y así estabilizar dicha estructura. Este tipo de interacciones es más probable que suceda en los dímero syn-Rsyn (entre los grupos $\mathrm{OH}$ de los Ca de las cadenas) y anti-Rtrans (donde un puente de $\mathrm{H}$ puede formarse entre el grupo $\mathrm{OH}$ del $\mathrm{C} \beta$ y otro del $\mathrm{C} \alpha^{\prime}$ o el HN5 del otro monómero). Por este motivo, podrían proponerse como estructuras más probables los dímeros anti-Rtrans y syn-Rsyn. Igualmente, es necesario aclarar que no hay evidencia suficiente para confirmar estas estructuras.

Con respecto a $\mathrm{H}_{2} \mathrm{Nep}$, se realizaron experimentos similares a los ya descriptos para $\mathrm{H}_{2}$ Bip. Como se detalló al comienzo de esta sección, todas las señales correspondientes al reactivo $\mathrm{H}_{2} \mathrm{Nep}$ aparecen en el intervalo de $\delta 3,9$ y 4,3. Consecuentemente, las señales de los dímeros también aparecen en ese intervalo, generando una gran cantidad de picos que dificultan seriamente la interpretación de los espectros. Por este motivo, el estudio se centró solo en $\mathrm{H}_{2} \mathrm{Bip}$.

Como ya se mencionó en la primera sección, los fotodímeros son inestables. Esto puede deberse a varios aspectos. Por un lado, la presencia de un heteroátomo $(\mathrm{N})$ en el azo-ciclobutano hace que la estructura sea menos estable comparado con un ciclo de cuatro carbonos. Por otro lado, factores estero-electrónicos como también la tensión inducida en la estructura cíclica dada, principalmente, por los múltiples anillos y por las cadenas poli-alcohólicas de gran tamaño presentes como sustituyentes, aportan mayor inestabilidad a las moléculas.

Teniendo en cuenta estos puntos y sabiendo que los productos de la reacción térmica de los dímeros son el reactivo intacto y otro monómero al que posiblemente se le adicionó una molécula de $\mathrm{H}_{2} \mathrm{O}$, la degradación de los dímeros podría estar dada por una reacción tipo retro-cicloadición [2+2] y la posterior hidrólisis por parte de uno de los monómeros.

Como ya se había mencionado, la solución irradiada y conservada un tiempo determinado en oscuridad se analizó por ${ }^{1} \mathrm{H}-\mathrm{RMN}$ (Figura 11.6.c). Estos espectros mostraron los picos correspondientes al reactivo regenerado y otras señales correspondientes a P1. Cabe destacar la existencia de dos grupos de señales correspondientes a P1. Esto sugiere que se estarían generando al menos dos productos. Dada la asimetría de las moléculas, este hecho sería compatible con más de una posibilidad de incorporación de la molécula de $\mathrm{H}_{2} \mathrm{O}$ cuando se produce la hidrólisis de 
los dímeros, según sea en C6' o en C7'. Además, estos dos grupos de señales tienen distinta intensidad, es decir que los dos productos hidratados podrían estar en distinta proporción.

\subsection{Conclusiones}

En este capítulo se investigó la estabilidad térmica de los fotodímeros generados a partir de $\mathrm{H}_{2}$ Bip y $\mathrm{H}_{2} \mathrm{Nep}$. Se observó que lo más probable es que se produzca una retrocicloadición [2+2], con la consiguiente formación de un producto que podría ser un monómero con la adición de una molécula de $\mathrm{H}_{2} \mathrm{O}$ y la regeneración de otra molécula de reactivo inicial. Además, se intentó establecer la estructura de los fotodímeros generados a partir de $\mathrm{H}_{2} \mathrm{Bip}$ y $\mathrm{H}_{2} \mathrm{Nep}$. Dada la complejidad de la reacción, la diversidad de productos formados y a la inestabilidad de los mismos, no fue posible aislar y caracterizar completamente los dímeros. Sin embargo, podría proponerse como estructuras más probables la formación de los dímeros anti-Rtrans y syn-Rsyn (Esquema 11.1). En el Esquema 11.2 se resumen los resultados de este capítulo.

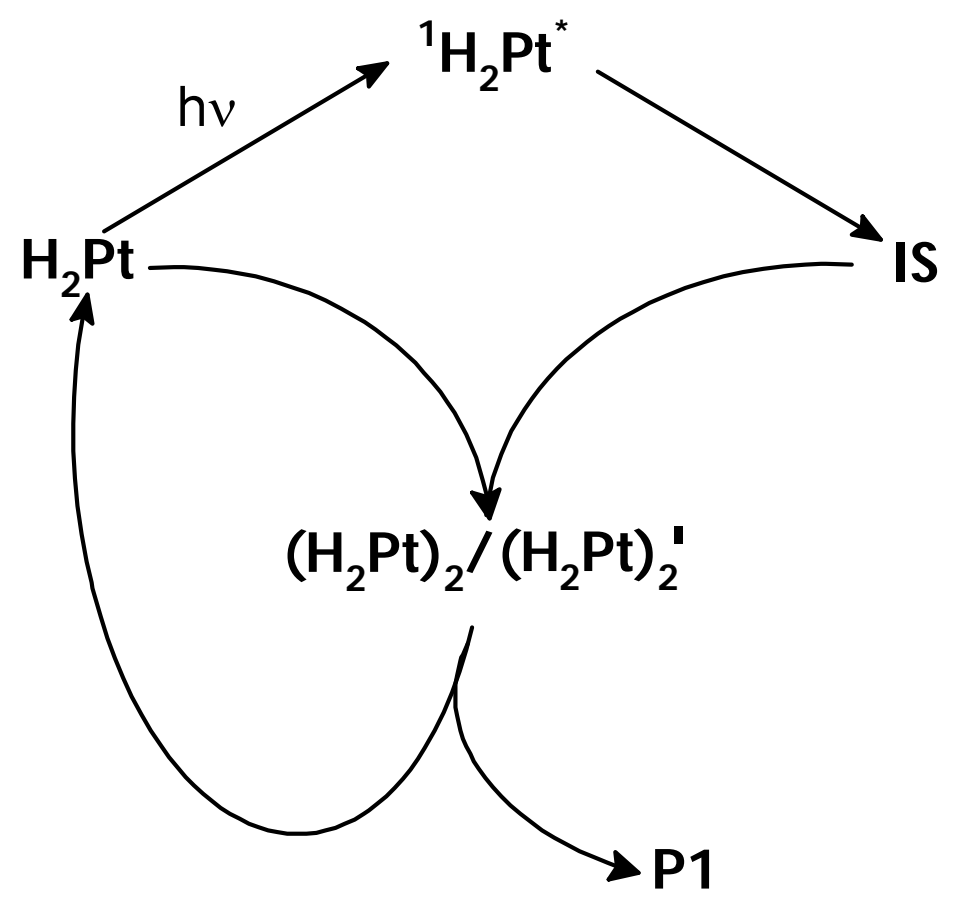

Esquema 11.2 Mecanismo propuesto de formación y degradación de los fotodímeros de $\mathrm{H}_{2}$ Bip y $\mathrm{H}_{2} \mathrm{Nep}$. 


\subsection{Referencias}

1. Y. Wang, Y. Li, Y. Wu and H. Yan, Mechanism of dihydroneopterin aldolase, FEBS Journal, 2007, 274, 2240-2252.

2. B. H. Jennings, S. Pastra-Landis and J. W. Lerman, Photosensitized dimerization of uracil, Photochemistry and Photobiology, 1972, 15, 479-491.

3. B. H. Jennings, S. C. Pastra and J. L. Wellington, Photosensitized dimerization of thymine, Photochemistry and Photobiology, 1970, 11, 215-226.

4. M. Poe and K. Hoogsteen, 5,6,7,8-Tetrahydrofolic acid. Conformation of the tetrahydropyrazine ring, J. Biol. Chem., 1978, 253, 543-546.

5. A. R. Katritzky, N. G. Akhmedov, E. M. Myshakin, A. K. Verma and C. Dennis Hall, Low-temperature 1H and 13C NMR spectra of N-substituted 1,2,3,4-tetrahydropyrazino[1,2-a]indoles, Magnetic Resonance in Chemistry, 2005, 43, 351-358. 


\section{Conclusiones Generales}

Las pterinas son compuestos heterocíclicos muy distribuidos en la naturaleza que pueden existir en distintos estados de oxidación, ya sea totalmente oxidados (pterinas aromáticas u oxidadas) o parcialmente reducidos (7,8-dihidropterinas y 5,6,7,8tetrahidropterinas). En solución acuosa las pterinas presentan un único equilibrio ácidobase relevante desde el punto de vista biológico, que involucra un grupo amida (forma ácida) y un grupo fenolato (forma alcalina), con un pKa de aproximadamente 8 para derivados aromáticos, y cercano a 10 para los reducidos (Esquema 1). Asimismo las pterinas poseen generalmente un sustituyente en el C6, el cual puede ser de tamaño y naturaleza química muy diversos afectando las propiedades fisicoquímicas y biológicas (Tabla 1).<smiles>[R]c1cnc2nc(N)nc([O-])c2n1</smiles>

forma ácida forma alcalina

Esquema 1 Equilibrio ácido-base del anillo pterínico en soluciones acuosas.<smiles>[R]c1cnc2nc(N)[nH]c(=O)c2n1</smiles><smiles>[R]C1=Nc2c(nc(N)[nH]c2=O)NC1</smiles>

\begin{tabular}{ccc}
\hline $\mathbf{R}$ & Pterina oxidada & Dihidroderivado \\
\hline$-(\mathrm{CHOH})_{2}-\mathrm{CH}_{2} \mathrm{OH}$ & neopterina (Nep) & 7,8-dihidroneopterina $\left(\mathrm{H}_{2} \mathrm{Nep}\right)$ \\
$-(\mathrm{CHOH})_{2}-\mathrm{CH}_{3}$ & biopterina (Bip) & 7,8-dihidrobiopterina $\left(\mathrm{H}_{2} \mathrm{Bip}\right)$ \\
$-\mathrm{CHO}$ & 6-formilpterina (Fop) & - \\
$-\mathrm{CH}_{2} \mathrm{OH}$ & 6-hidroximetilpterina (Hmp) & - \\
$-\mathrm{COOH}$ & 6-carboxipterina (Cap) & - \\
$-\mathrm{H}$ & pterina (Ptr) & - \\
\hline
\end{tabular}

Tabla 1 Nomenclatura y estructura química de pterinas oxidadas y 7,8-dihidropterinas.

Las pterinas se encuentran en la naturaleza principalmente como pigmentos en insectos y cofactores enzimáticos de muchas reacciones del metabolismo de aminoácidos y ácidos nucleicos. También existen derivados cuyas funciones aún no han 
sido completamente dilucidadas y podrían participar en procesos de fotorrecepción, transducción de señales y protección contra el estrés oxidativo.

La fotoquímica de las pterinas comenzó a despertar interés cuando se descubrió su participación en procesos fotobiológicos. Por otro lado, se ha demostrado que algunos derivados se acumulan en la piel de pacientes que sufren vitiligo, enfermedad cutánea que cursa con déficit de pigmentación, debido a la interrupción total o parcial de la síntesis de melanina, el pigmento natural de la piel del ser humano. El déficit de melanina hace que el tejido afectado no pueda filtrar la radiación UV y, por ende, los procesos fotoquímicos que involucran compuestos presentes en la piel adquieren gran interés. En este trabajo de Tesis se estudió el comportamiento fotoquímico en solución acuosa de dos compuestos que se acumulan en la piel de los pacientes que sufren vitiligo biopterina (Bip) y su dihidroderivado, 7,8-dihidrobiopterina ( $\left.\mathrm{H}_{2} \mathrm{Bip}\right)$. Además se estudiaron otros dos compuestos relacionados estructuralmente con éstos: neopterina (Nep) y 7,8-dihidroneopterina ( $\mathrm{H}_{2} \mathrm{Nep}$ ).

Los estudios se realizaron en solución acuosa bajo irradiación UV-A (320-400 $\mathrm{nm})$, a pH fisiológico ( 7) para los dihidroderivados, y a $\mathrm{pH}=5,5$ ó 10,5 para las pterinas aromáticas. De esta manera, se logró tener en solución una sola forma ácidobase de cada pterina. Para las distintas reacciones fotoquímicas estudiadas se caracterizaron/identificaron los productos de reacción, se obtuvieron rendimientos cuánticos y se investigaron los mecanismos de reacción. Además se estudió la estabilidad en la oscuridad de los fotoproductos generados. A continuación se detallan las conclusiones generales más relevantes y en el esquema final se resumen las reacciones fotoquímicas y térmicas investigadas.

e Bip y Nep se consumen cuando sus soluciones acuosas aireadas son irradiadas con radiación UV-A, generando como productos 6- formilpterina (Fop) y peróxido de hidrógeno $\left(\mathrm{H}_{2} \mathrm{O}_{2}\right)$. A su vez, en condiciones de anaerobiosis, se genera un intermediario rojo que fue identificado como 6-formil-5,8-dihidropterina. Este intermediario, que es también formado fotoquímicamente por 6hidroximetilpterina (Hmp), reacciona rápidamente con $\mathrm{O}_{2}$ para generar Fop y $\mathrm{O}_{2}{ }^{\bullet-}$, que luego se transforma en $\mathrm{H}_{2} \mathrm{O}_{2}$. 6-Formil-5,8-dihidropterina se genera a partir de los estados excitados tripletes de los compuestos estudiados y es inestable también en ausencia de $\mathrm{O}_{2}$ descomponiéndose lentamente en una serie de productos entre los cuales se encuentra nuevamente a Fop. Por otro lado, se calcularon los rendimientos cuánticos de consumo de reactivo ( $\left.\Phi_{-\mathrm{R}}\right)$ para ambas formas ácido- 
base y en distintas concentraciones de $\mathrm{O}_{2}$ (Tabla 2). Para las dos pterinas oxidadas, los $\Phi_{\text {-R }}$ se incrementan con la disminución de la concentración de $\mathrm{O}_{2}$ y son mayores para las formas alcalinas que para las formas ácidas. La disminución de los valores de $\Phi_{-\mathrm{R}}$ con la concentración de $\mathrm{O}_{2}$ se atribuye a la desactivación de los estados tripletes de las pterinas por esa especie.

\begin{tabular}{cccccccc}
\hline$\Phi_{\text {- }}$ & $\mathbf{p H}=\mathbf{7 , 0}$ & \multicolumn{3}{c}{$\mathbf{p H}=\mathbf{5 , 5}$} \\
\hline & & Ar & Aire & $\mathrm{O}_{2}$ & Ar & Aire & $\mathrm{O}_{2}$ \\
Bip & - & $0,10 \pm 0,01$ & $0,037 \pm 0,003$ & $0,024 \pm 0,003$ & $0,18 \pm 0,02$ & $0,12 \pm 0,01$ & $0,07 \pm 0,01$ \\
Nep & - & $0,11 \pm 0,01$ & $0,044 \pm 0,003$ & $0,018 \pm 0,002$ & $0,16 \pm 0,03$ & $0,11 \pm 0,01$ & $0,069 \pm 0,005$ \\
$\mathbf{H}_{2}$ Bip & $0,05 \pm 0,01$ & - & - & - & - & - & - \\
$\mathbf{H}_{2}$ Nep & $0,038 \pm 0,005$ & - & - & - & - & - & - \\
\hline
\end{tabular}

\section{Tabla 2 Rendimiento cuántico de las pterinas estudiadas.}

c $\mathrm{H}_{2} \mathrm{Nep}$ y $\mathrm{H}_{2}$ Bip se consumen fotoquímicamente para generar cada una dos dímeros con peso molecular exactamente igual al doble del peso molecular de cada reactivo. Esta reacción se genera a partir del estado excitado singlete del reactivo, el cual forma un intermediario que reacciona con una molécula del dihidroderivado en estado basal. Este proceso es independiente de la concentración de $\mathrm{O}_{2}$ disuelto en el medio y de la concentración inicial de reactivo. Se propusieron estructuras probables para los fotodímeros, las cuales comprenderían la formación de un azo-ciclobutano formado por N5-C6 y C6-C7 de cada monómero. Asimismo, los dímeros formados son inestable en oscuridad, produciendo la regeneración del reactivo inicial y formando otro producto, el cual posiblemente sea una molécula de reactivo que incorporó una molécula de $\mathrm{H}_{2} \mathrm{O}$.

e Por su parte, $\mathrm{H}_{2}$ Bip además de fotodimerizar, en presencia de $\mathrm{O}_{2}$ se oxida generando su análogo aromático, Bip. Esta vía, que es despreciable en un primer momento, adquiere importancia a medida que transcurre el proceso fotoquímico. Esto se debe a que se produce una aceleración en la vía oxidativa, generada por la fotosensibilización de $\mathrm{H}_{2}$ Bip por parte de Bip y otras pterinas oxidadas, a través de un mecanismo de transferencia de electrones desde $\mathrm{H}_{2}$ Bip hacia el triplete excitado de Bip. $\mathrm{H}_{2} \mathrm{Nep}$ también sufre una fotosensibilización por parte de pterinas oxidadas, pero en este caso, el producto mayoritario es un compuesto que no pudo ser identificado. Este producto no absorbe a la longitud de onda de irradiación, motivo por el cual la fotosensibilización no interfiere en la fotoquímica directa del reactivo. 
e. La oxidación fotoquímica de $\mathrm{H}_{2}$ Bip a Bip no había sido probada hasta el momento y podría explicar la formación de este último compuesto, que es generador de especies reactivas de oxígeno y citotóxico para los melanocitos, en la piel afectada por vitiligo. Por otro lado, se demostró, por primera vez, la formación de anión superóxido y $\mathrm{H}_{2} \mathrm{O}_{2}$ en las fotooxidaciones de $\mathrm{H}_{2}$ Bip y Bip lo que podría contribuir al estrés oxidativo demostrado en las zonas que sufren falta de pigmentación en la piel. 


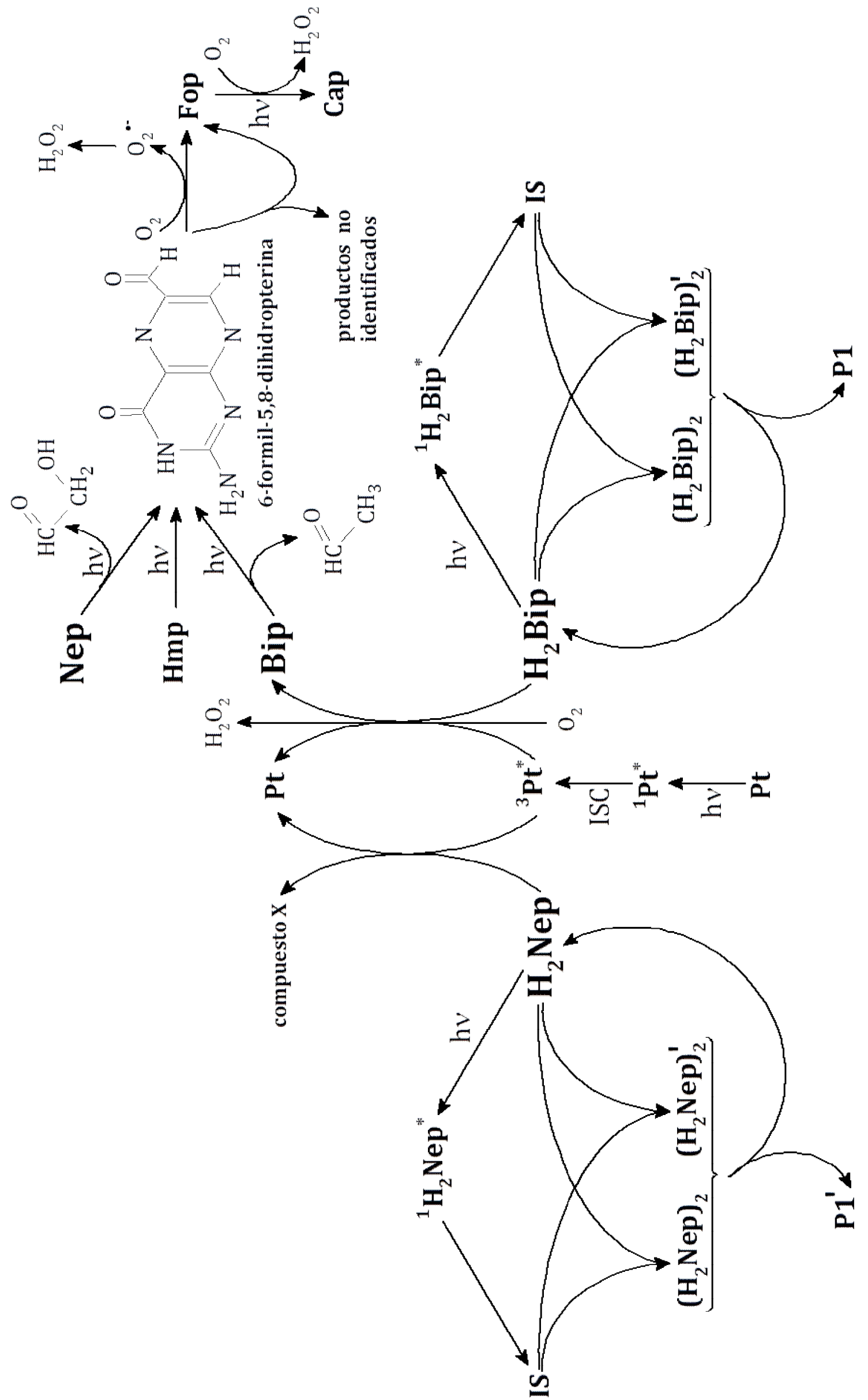

